Supporting Information

\title{
Tandem C-H Transformations by a Single Iridium Catalyst: Direct Access to Indoles and Indolines from $o$-Alkyl- $N$-methylanilines
}

Toshimichi Ohmura,* Kaito Yagi, Satoshi Kusaka, Michinori Suginome*

Department of Synthetic Chemistry and Biological Chemistry, Graduate School of Engineering, Kyoto University, Katsura, Nishikyo-ku, Kyoto 615-8510, Japan

ohmura@sbchem.kyoto-u.ac.jp; suginome@sbchem.kyoto-u.ac.jp

\section{Contents}

1. General ----- S2

2. Materials ----- S2

3. Preparation of Substrates 1a-r, 4, 6, 8, 3a, 9, and 11a ----- S3

4. Reaction Conditions (Table 1) ----- S16

5. Iridium-Catalyzed Dehydrogenation-Intramolecular Dehydrogenative $\mathrm{C}-\mathrm{H} / \mathrm{C}-\mathrm{H}$ Coupling of 1 (Scheme 2) ----- S19

6. Reactions of Other Anilines and an Indoline (Figure 1) ----- S31

7. Reactions of D-Labeling Substrates (Figure 2) ----- S34

8. Application for the Isomer-Selective Synthesis of Borylindoles (Scheme 4-i) ----- S41

9. Application for the Isomer-Selective Synthesis of Bromoindoles (Scheme 4-ii) ----- S43

10. Asymmetric Conversion of $\mathbf{2 0}$ Affording Enantioenriched Indolines 21 (Table 2) $----\mathrm{S} 45$

11. References ----- S54

12. ${ }^{1} \mathrm{H}$ and ${ }^{13} \mathrm{C}$ NMR Spectra of New Compounds and Key Compounds ----- S55 


\section{General}

All iridium-catalyzed reactions were performed in glovebox under an atmosphere of nitrogen with magnetic stirring. Other reactions were also carried out under an atmosphere of nitrogen with magnetic stirring unless otherwise noted. Materials were weighted by an electric balance, Sartorius CPA225D or Shimadzu AP225WD (readability: $0.01 \mathrm{mg}$ ). Column chromatography was performed with SiliaFlash (SILICYCLE, pH 7.0, 40-63 $\mu \mathrm{m}, 60 \AA$ ). Gas chromatography (GC) was performed by Shimadzu GC-2010 with Agilent J\&W GC Column DB-1 ( $\phi 0.32 \mathrm{~mm} \mathrm{x}$ $15 \mathrm{~m})$. GCMS analyses were performed by Shimadzu GCMS-QP2020 with Agilent J\&W GC Column DB-1 ( $\phi 0.32$ mm x 15 m). ${ }^{1} \mathrm{H}$ NMR spectra were recorded on a Varian 400-MR (399.89 $\mathrm{MHz})$ spectrometer. ${ }^{13} \mathrm{C}$ NMR spectra were recorded on a Varian 400-MR (100.55 MHz) spectrometer. ${ }^{11}$ B NMR spectra were recorded on a Varian 400-MR (128.30 MHz) spectrometer. ${ }^{1} \mathrm{H}$ NMR data were reported as follows: chemical shifts in ppm downfield from tetramethylsilane, multiplicity ( $\mathrm{s}=$ singlet, $\mathrm{d}=$ doublet, $\mathrm{t}=$ triplet, $\mathrm{q}=$ quartet, and $\mathrm{m}=$ multiplet $)$, coupling constant $(J)$, and integration. The chemical shifts of ${ }^{13} \mathrm{C}$ and ${ }^{11} \mathrm{~B}$ NMR spectra were reported in ppm downfield from tetramethylsilane $\left({ }^{13} \mathrm{C}\right)$ and $\mathrm{BF}_{3} \cdot \mathrm{OEt}_{2}\left({ }^{11} \mathrm{~B}\right)$, respectively. High resolution mass spectra (HRMS) were recorded on a Thermo Scientific Exactive Plus (APCI, ESI) spectrometer.

\section{Materials}

Solvents for Reactions: Mesitylene and 1,4-dioxane were distilled over benzophenone ketyl. Toluene (dehydrated, Super plus, Kanto), tetrahydrofuran (THF, dehydrated, Super plus, Kanto), and diethyl ether (dehydrated, Kanto) were purchased and used without further purification. Methylcyclohexane and hexane were distilled over calcium hydride and degassed. DMF, MeCN, and AcOEt were used as received.

Catalysts and Ligands: $\left[\operatorname{IrCl}\left(\mathrm{C}_{2} \mathrm{H}_{4}\right)_{2}\right]_{2}$ was prepared according to the procedure reported previously ${ }^{1}$ and stored at $-30{ }^{\circ} \mathrm{C}$ under nitrogen atmosphere. L1 [(S)-SEGPHOS, TCI], L2 [(S)-DM-SEGPHOS, TCI], L3 [(S)-DTBM-SEGPHOS, TCI], L4 [(S)-MeOBIPHEP, Aldrich], L5 $[(S)$-DTBM-MeOBIPHEP, Aldrich], L6 [(S)-BINAP, Aldrich], and L7 [(S)-DTBM-BINAP, Wako] were used as received from commercial source. 


\section{Preparation of Substrates 1a-q, 4, 6, 8, 3a, 9, and 11a}

\section{2-Ethyl- $N, N$-dimethylaniline (1a)}

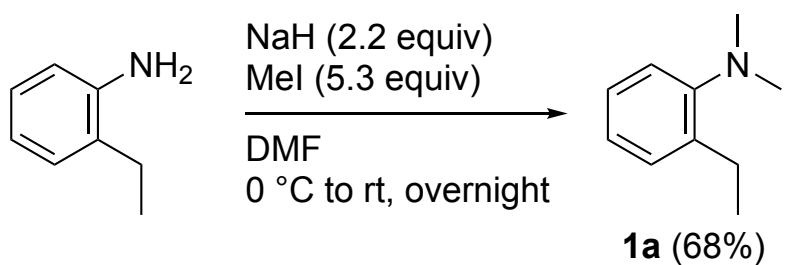

An oven dried $200 \mathrm{~mL}$ three-neck flask, equipped with a magnetic stirring bar, a rubber septum, and a three-way stopcock, was charged with $\mathrm{NaH}$ (ca. 60\% dispersion in mineral oil, 1.0 g, $25 \mathrm{mmol})$. The flask was evacuated and backfilled with nitrogen. DMF (20 mL) and 2-ethylaniline (Wako, $1.36 \mathrm{~g}, 11 \mathrm{mmol}$ ) were added to the flask, and the mixture was cooled to 0 ${ }^{\circ} \mathrm{C}$ by ice/water bath. Methyl iodide $(8.43 \mathrm{~g}, 59.5 \mathrm{mmol})$ was added slowly to the flask. The ice/water bath was removed, and the mixture was stirred overnight at room temperature. Hexane $(30 \mathrm{~mL})$, AcOEt $(30 \mathrm{~mL})$, and water $(60 \mathrm{~mL})$ were added to the flask. The organic phase was collected, washed with water $(60 \mathrm{~mL} \times 4)$ and brine $(60 \mathrm{~mL})$, and dried over anhydrous magnesium sulfate. The crude product was purified by column chromatography on silica gel (SiliaFlash; eluent: hexane:Et $2 \mathrm{O}=10: 1)$ and Kugelrhor distillation. 1a (1.13 g, $7.57 \mathrm{mmol}, 68 \%$ yield) was obtained as a colorless oil. 1a: ${ }^{1} \mathrm{H}$ NMR $\left(400 \mathrm{MHz}, \mathrm{CDCl}_{3}\right) \delta 7.28(\mathrm{dd}, J=7.2,1.6 \mathrm{~Hz}$, $1 \mathrm{H}), 7.22(\mathrm{td}, J=8.0,1.6 \mathrm{~Hz}, 1 \mathrm{H}), 7.14(\mathrm{dd}, J=8.0,1.6 \mathrm{~Hz}, 1 \mathrm{H}), 7.08(\mathrm{td}, J=7.2,1.6 \mathrm{~Hz}, 1 \mathrm{H})$, 2.80 (q, $J=7.6 \mathrm{~Hz}, 2 \mathrm{H}), 2.74(\mathrm{~s}, 6 \mathrm{H}), 1.31(\mathrm{t}, J=7.6 \mathrm{~Hz}, 3 \mathrm{H}) .{ }^{13} \mathrm{C}$ NMR $\left(101 \mathrm{MHz}, \mathrm{CDCl}_{3}\right) \delta$ 152.6, 138.8, 129.0, 126.4, 123.4, 119.2, 45.2, 23.6, 14.9. HRMS (APCI, positive) $\mathrm{m} / z$ calcd for $\mathrm{C}_{10} \mathrm{H}_{16} \mathrm{~N}^{+}[\mathrm{M}+\mathrm{H}]^{+}: 150.1277$, found: 150.1278 .

\section{2-Ethyl- $N, N, 6$-trimethylaniline (1b)}

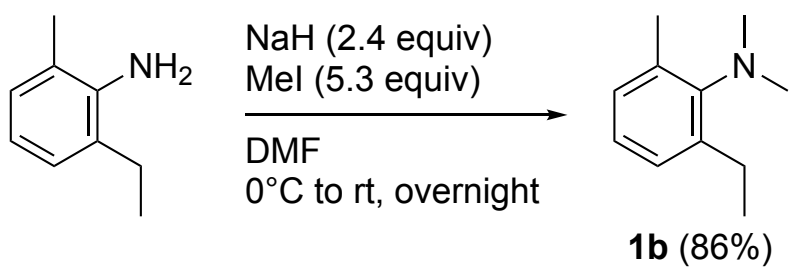

According to the procedure given for $1 \mathbf{a}, \mathbf{1 b}(1.38 \mathrm{~g}, 8.45 \mathrm{mmol}, 86 \%$ yield $)$ was prepared as a colorless oil from 2-ethyl-6-methylaniline (TCI, $1.33 \mathrm{~g}, 9.83 \mathrm{mmol}$ ). 1b: ${ }^{1} \mathrm{H}$ NMR (400 MHz, $\left.\mathrm{CDCl}_{3}\right) \delta 7.01-7.12(\mathrm{~m}, 3 \mathrm{H}), 2.88(\mathrm{~s}, 6 \mathrm{H}), 2.73(\mathrm{q}, J=7.6 \mathrm{~Hz}, 2 \mathrm{H}), 2.36(\mathrm{~s}, 3 \mathrm{H}), 1.27(\mathrm{t}, J=7.6$ 
$\mathrm{Hz}, 3 \mathrm{H}) .{ }^{13} \mathrm{C} \mathrm{NMR}\left(101 \mathrm{MHz}, \mathrm{CDCl}_{3}\right) \delta 149.3,143.9,137.7,129.0,127.0,125.3,43.1,25.2$, 19.3, 15.7. HRMS (APCI, positive) $m / z$ calcd for $\mathrm{C}_{11} \mathrm{H}_{18} \mathrm{~N}^{+}[\mathrm{M}+\mathrm{H}]^{+}$: 164.1434, found: 164.1434.

\section{2,6-Diethyl- $N, N$-dimethylaniline (1c)}

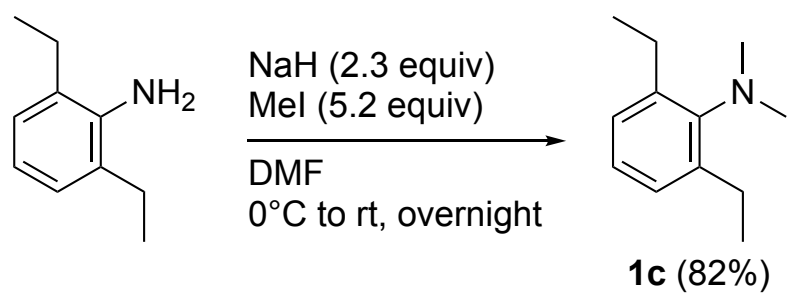

According to the procedure given for $1 \mathrm{a}, 1 \mathrm{c}(1.50 \mathrm{~g}, 10.1 \mathrm{mmol}, 82 \%$ yield $)$ was prepared as a colorless oil from 2,6-diethylaniline (TCI, $1.46 \mathrm{~g}, 8.24 \mathrm{mmol}) .1 \mathrm{c}:{ }^{1} \mathrm{H} \mathrm{NMR}\left(400 \mathrm{MHz}, \mathrm{CDCl}_{3}\right) \delta$ 7.06-7.14 (m, 3H), $2.88(\mathrm{~s}, 6 \mathrm{H}), 2.71$ (q, $J=7.6 \mathrm{~Hz}, 4 \mathrm{H}), 1.27(\mathrm{t}, J=7.6 \mathrm{~Hz}, 6 \mathrm{H}) .{ }^{13} \mathrm{C} \mathrm{NMR}(101$ $\mathrm{MHz}_{\mathrm{CDCl}}$ ) $\delta 148.9,144.2,127.1,125.7,43.8,25.1,15.7$. HRMS (APCI, positive) $\mathrm{m} / z$ calcd for $\mathrm{C}_{12} \mathrm{H}_{20} \mathrm{~N}^{+}[\mathrm{M}+\mathrm{H}]^{+}: 178.1590$, found: 178.1590 .

\section{$N, N$-dimethyl-2-propylaniline (1d)}

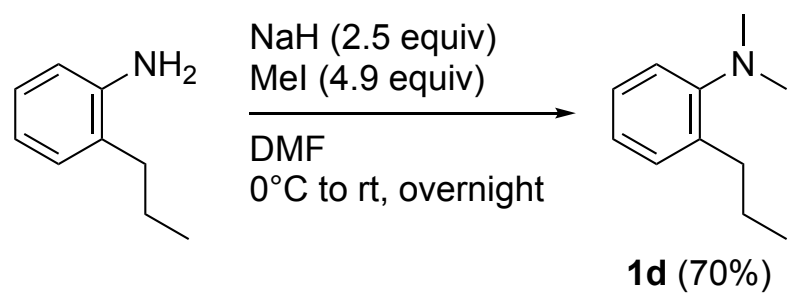

According to the procedure given for 1a, 1d (1.36 g, $10.1 \mathrm{mmol}, 70 \%$ yield) was prepared as a colorless oil from 2-propylaniline (TCI, $1.16 \mathrm{~g}, 7.11 \mathrm{mmol}) .1 \mathrm{dd}:{ }^{1} \mathrm{H} \mathrm{NMR}\left(400 \mathrm{MHz}, \mathrm{CDCl}_{3}\right) \delta$ 7.13-7.23 (m, 2H), $7.10(\mathrm{dd}, J=8.0,1.2 \mathrm{~Hz}, 1 \mathrm{H}), 7.02(\mathrm{td}, J=7.2,1.6 \mathrm{~Hz}, 1 \mathrm{H}), 2.69(\mathrm{~s}, 6 \mathrm{H})$, 2.65-2.71 (m, 2H), 1.68 (sextet, $J=7.6 \mathrm{~Hz}, 2 \mathrm{H}), 1.00(\mathrm{t}, J=7.6 \mathrm{~Hz}, 3 \mathrm{H}) .{ }^{13} \mathrm{C} \mathrm{NMR}(101 \mathrm{MHz}$, $\left.\mathrm{CDCl}_{3}\right) \delta 152.8,137.6,129.7,126.5,123.4,119.4,45.3,32.9,23.9,14.5$. HRMS (APCI, positive) $m / z$ calcd for $\mathrm{C}_{11} \mathrm{H}_{18} \mathrm{~N}^{+}[\mathrm{M}+\mathrm{H}]^{+}: 164.1434$, found: 164.1434 . 


\section{2-Butyl- $N, N$-dimethylaniline (1e)}

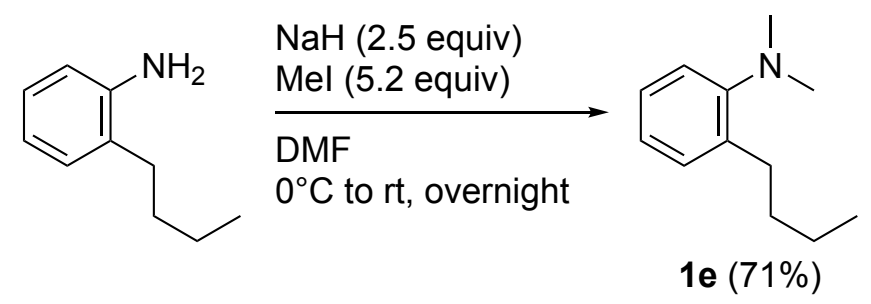

According to the procedure given for $1 \mathrm{a}, 1 \mathrm{e}(1.29 \mathrm{~g}, 7.28 \mathrm{mmol}, 71 \%$ yield) was prepared as a colorless oil from 2-butylaniline (TCI, $1.52 \mathrm{~g}, 10.2 \mathrm{mmol}) .1 \mathrm{e}:{ }^{1} \mathrm{H}$ NMR $\left(400 \mathrm{MHz}, \mathrm{CDCl}_{3}\right) \delta$ $7.24(\mathrm{dd}, J=7.2,1.6 \mathrm{~Hz}, 1 \mathrm{H}), 7.16-7.22(\mathrm{~m}, 1 \mathrm{H}), 7.13$ [dd (AB pattern), $J=8.0,1.6 \mathrm{~Hz}, 1 \mathrm{H}$ ], $7.05(\mathrm{td}, J=7.2,1.6 \mathrm{~Hz}, 1 \mathrm{H}), 2.72(\mathrm{~s}, 6 \mathrm{H}), 2.70-2.77(\mathrm{~m}, 2 \mathrm{H}), 1.63-1.72(\mathrm{~m}, 2 \mathrm{H}), 1.45$ (sextet, $J$ $=7.6 \mathrm{~Hz}, 2 \mathrm{H}), 1.00(\mathrm{t}, J=7.6 \mathrm{~Hz}, 3 \mathrm{H}) .{ }^{13} \mathrm{C} \mathrm{NMR}\left(101 \mathrm{MHz}, \mathrm{CDCl}_{3}\right) \delta 152.8,137.7,129.7,126.4$, 123.4, 119.4, 45.3, 33.0, 30.4, 23.1, 14.2. HRMS (APCI, positive) $m / z$ calcd for $\mathrm{C}_{12} \mathrm{H}_{20} \mathrm{~N}^{+}[\mathrm{M}+$ $\mathrm{H}]^{+}: 178.1590$, found: 178.1590 .

\section{2-Ethyl- $N, N, 4-t$ trimethylaniline (1f) and 4-Bromo-2-ethyl- $N, N$-dimethylaniline (1r)}<smiles>CCc1ccccc1N(C)C</smiles>

$1 \mathrm{a}$
NBS (1.1 equiv) $\mathrm{NH}_{4} \mathrm{OAc}(14 \mathrm{~mol} \%)$

$\mathrm{MeCN}$ $\mathrm{rt}, 10 \mathrm{~min}$

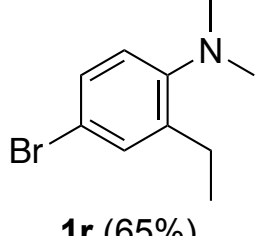

$\operatorname{1r}(65 \%)$
1. s-BuLi (1.2 equiv) THF/cyclohexane $-78^{\circ} \mathrm{C}, 2 \mathrm{~h}$

2. Mel (2.0 equiv) THF/cyclohexane $-78^{\circ} \mathrm{C}$ to rt, overnight

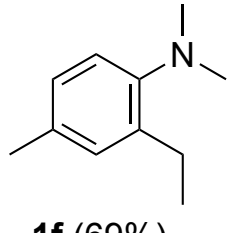

$1 f(69 \%)$

The brominaiton of 1 a was carried out according to the procedure reported. ${ }^{2}$ A $50 \mathrm{~mL}$ flask, equipped with a magnetic stirring bar, was charged with 1a (0.723 g, $4.84 \mathrm{mmol})$, ammonium acetate $(54 \mathrm{mg}, 0.701 \mathrm{mmol})$ and $\mathrm{MeCN}(10 \mathrm{~mL}) . \mathrm{N}$-Bromosuccinimide (NBS, nacalai, $0.900 \mathrm{~g}$, $5.06 \mathrm{mmol}$ ) was added slowly to the flask under air, and the resulting mixture was stirred for 10 min. The organic materials were extracted with $\mathrm{CH}_{2} \mathrm{Cl}_{2}(30 \mathrm{~mL} \times 3)$, washed with brine $(50 \mathrm{~mL} \times$ 1), and dried over anhydrous sodium sulfate. The crude product was purified by column chromatography on silica gel (SiliaFlash; eluent: hexane:Et ${ }_{2} \mathrm{O}=20: 1$ ) and Kugelrhor distillation. 1r $\left(0.721 \mathrm{~g}, 3.16 \mathrm{mmol}, 65 \%\right.$ yield) was obtained as a colorless oil. 1r: ${ }^{1} \mathrm{H}$ NMR (400 MHz, $\left.\mathrm{CDCl}_{3}\right) \delta 7.33(\mathrm{~d}, J=2.4 \mathrm{~Hz}, 1 \mathrm{H}), 7.26(\mathrm{dd}, J=8.4,2.4 \mathrm{~Hz}, 1 \mathrm{H}), 6.94(\mathrm{~d}, J=8.4 \mathrm{~Hz}, 1 \mathrm{H}), 2.70$ $(\mathrm{q}, J=7.6 \mathrm{~Hz}, 2 \mathrm{H}), 2.66(\mathrm{~s}, 6 \mathrm{H}), 1.25(\mathrm{t}, J=7.6 \mathrm{~Hz}, 3 \mathrm{H}) .{ }^{13} \mathrm{C} \mathrm{NMR}\left(101 \mathrm{MHz}, \mathrm{CDCl}_{3}\right) \delta 151.7$, 
141.2, 131.9, 129.3, 121.1, 116.4, 45.1, 23.5, 14.7. HRMS (APCI, positive) $\mathrm{m} / z$ calcd for $\mathrm{C}_{10} \mathrm{H}_{15} \mathrm{BrN}^{+}[\mathrm{M}+\mathrm{H}]^{+}:$228.0382, found: 228.0383 .

An oven dried $100 \mathrm{~mL}$ two-neck flask, equipped with a magnetic stirring bar, a rubber septum, and a three-way stopcock, was evacuated and backfilled with nitrogen. THF (10 mL) and 1r (0.589 $\mathrm{g}, 2.58 \mathrm{mmol})$ were added to the flask, and the mixture was cooled to $-78{ }^{\circ} \mathrm{C}$ by dry ice/acetone bath. $s$-BuLi (Kanto, 3.0 mL, 1.0 M solution in cyclohexane) was added slowly to the flask, and the resulting mixture was stirred for $2 \mathrm{~h}$ at $-78^{\circ} \mathrm{C}$. Methyl iodide $(0.729 \mathrm{~g}, 5.14 \mathrm{mmol})$ was added slowly to the flask. The dry ice/acetone bath was removed, and the mixture was stirred overnight at room temperature. Water $(20 \mathrm{~mL})$ was added to the flask. The organic materials were extracted with $\mathrm{Et}_{2} \mathrm{O}(15 \mathrm{~mL}$ x 3), washed with brine (30 mL x 1), and dried over anhydrous magnesium sulfate. The crude product was purified by column chromatography on silica gel (SiliaFlash; eluent: hexane: $\left.\mathrm{Et}_{2} \mathrm{O}=20: 1\right), \mathrm{GPC}$, and Kugelrhor distillation. $1 f(0.292$ g, 1.79 mmol,

$69 \%$ yield) was obtained as a colorless oil. 1f: ${ }^{1} \mathrm{H} \mathrm{NMR}\left(400 \mathrm{MHz}, \mathrm{CDCl}_{3}\right) \delta 6.99-7.10(\mathrm{~m}, 3 \mathrm{H})$, $2.76(\mathrm{q}, J=7.6 \mathrm{~Hz}, 2 \mathrm{H}), 2.70(\mathrm{~s}, 6 \mathrm{H}), 2.34(\mathrm{~s}, 3 \mathrm{H}), 1.29(\mathrm{t}, J=7.6 \mathrm{~Hz}, 3 \mathrm{H}) .{ }^{13} \mathrm{C} \mathrm{NMR}(101 \mathrm{MHz}$, $\left.\mathrm{CDCl}_{3}\right) \delta 150.2,138.9,132.9,129.8,127.0,119.3,45.5,23.5,21.0,15.1$. HRMS (APCI, positive) $m / z$ calcd for $\mathrm{C}_{11} \mathrm{H}_{18} \mathrm{~N}^{+}[\mathrm{M}+\mathrm{H}]^{+}: 164.1434$, found: 164.1434 .

\section{4-Acetyl-2-ethyl- $N, N$-dimethylaniline (1g)}

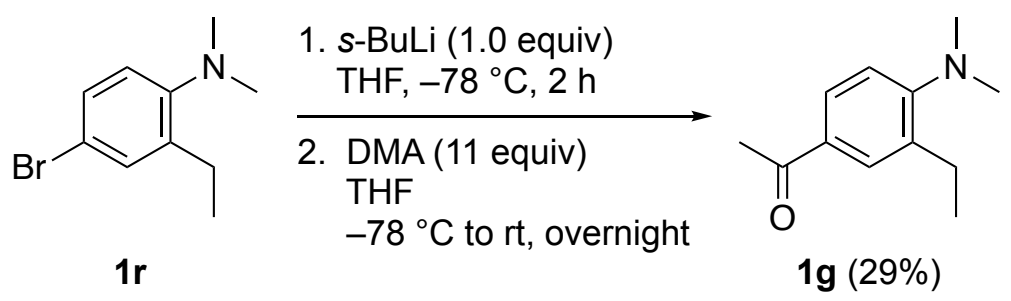

An oven dried $100 \mathrm{~mL}$ two-neck flask, equipped with a magnetic stirring bar, a rubber septum, and a three-way stopcock, was evacuated and backfilled with nitrogen. THF (8 mL) and 1r $(0.448 \mathrm{~g}, 1.96 \mathrm{mmol})$ were added to the flask, and the mixture was cooled to $-78{ }^{\circ} \mathrm{C}$ by dry ice/acetone bath. $s$-BuLi (1.0 M solution in cyclohexane, $2.0 \mathrm{~mL}, 2.0 \mathrm{mmol})$ was added slowly to the flask, and the resulting mixture was stirred for $2 \mathrm{~h}$ at $-78^{\circ} \mathrm{C} . N, N$-Dimethylacetamide (DMA, $2.0 \mathrm{~mL}, 22 \mathrm{mmol}$ ) was added slowly to the flask. The dry ice/acetone bath was removed, and the mixture was stirred overnight at room temperature. Water $(20 \mathrm{~mL})$ was added to the flask. The 
organic materials were extracted with $\mathrm{Et}_{2} \mathrm{O}(20 \mathrm{~mL} \times 3)$, washed with brine $(50 \mathrm{~mL} \times 1)$, and dried over anhydrous magnesium sulfate. The crude product was purified by column chromatography on silica gel (SiliaFlash; eluent: hexane: $\left.\mathrm{Et}_{2} \mathrm{O}=90: 10\right)$ and Kugelrhor distillation. 1 g (0.108 g, $0.565 \mathrm{mmol}, 29 \%$ yield) was obtained as a colorless oil. 1g: ${ }^{1} \mathrm{H}$ NMR (400 MHz, $\left.\mathrm{CDCl}_{3}\right) \delta 7.84(\mathrm{~d}, J=2.0 \mathrm{~Hz}, 1 \mathrm{H}), 7.74(\mathrm{dd}, J=8.4,2.0 \mathrm{~Hz}, 1 \mathrm{H}), 7.01(\mathrm{~d}, J=8.4 \mathrm{~Hz}, 1 \mathrm{H}), 2.77(\mathrm{~s}$, $6 \mathrm{H}), 2.73(\mathrm{q}, J=7.6 \mathrm{~Hz}, 2 \mathrm{H}), 2.55(\mathrm{~s}, 3 \mathrm{H}), 1.29(\mathrm{t}, J=7.6 \mathrm{~Hz}, 3 \mathrm{H}) .{ }^{13} \mathrm{C} \mathrm{NMR}\left(101 \mathrm{MHz}, \mathrm{CDCl}_{3}\right)$ $\delta$ 197.6, 157.1, 137.1, 131.3, 129.6, 127.4, 117.9, 44.3, 26.5, 24.2, 14.6. HRMS (APCI, positive) $m / z$ calcd for $\mathrm{C}_{12} \mathrm{H}_{18} \mathrm{NO}^{+}[\mathrm{M}+\mathrm{H}]^{+}: 192.1383$, found: 192.1383 .

\section{4-Cyano-2-ethyl- $N, N$-dimethylaniline (1h)}

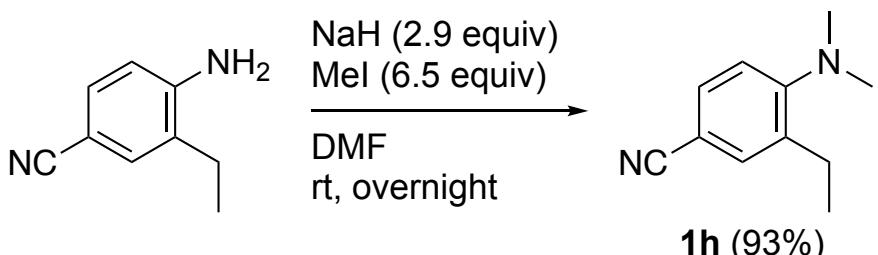

According to the procedure given for $1 \mathbf{a}, 1 \mathbf{h}(0.649 \mathrm{~g}, 3.72 \mathrm{mmol}, 93 \%$ yield $)$ was prepared as a colorless oil from 4-cyano-2-ethylaniline (Wako, $0.588 \mathrm{~g}, 4.02 \mathrm{mmol}$ ). 1h: ${ }^{1} \mathrm{H} \mathrm{NMR}(400 \mathrm{MHz}$, $\left.\mathrm{CDCl}_{3}\right) \delta 7.44(\mathrm{~d}, J=2.0 \mathrm{~Hz}, 1 \mathrm{H}), 7.39(\mathrm{dd}, J=8.0,2.0 \mathrm{~Hz}, 1 \mathrm{H}), 6.99(\mathrm{~d}, J=8.0 \mathrm{~Hz}, 1 \mathrm{H}), 2.74(\mathrm{~s}$, $6 \mathrm{H}), 2.68(\mathrm{q}, J=7.6 \mathrm{~Hz}, 2 \mathrm{H}), 1.24(\mathrm{t}, J=7.6 \mathrm{~Hz}, 3 \mathrm{H}) .{ }^{13} \mathrm{C}$ NMR $\left(101 \mathrm{MHz}, \mathrm{CDCl}_{3}\right) \delta 156.5$, 138.0, 132.8, 130.5, 119.9, 118.6, 104.8, 44.0, 23.8, 14.1. HRMS (ESI, positive) $\mathrm{m} / \mathrm{z}$ calcd for $\mathrm{C}_{11} \mathrm{H}_{15} \mathrm{~N}_{2}^{+}[\mathrm{M}+\mathrm{H}]^{+}:$175.1230, found: 175.1231 .

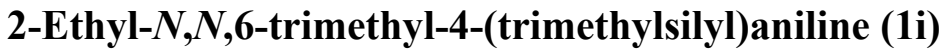

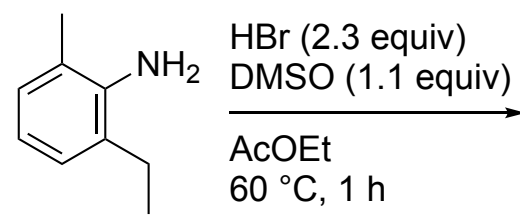

1. $s$-BuLi (1.3 equiv) THF, $-78{ }^{\circ} \mathrm{C}$

2. $\mathrm{Me}_{3} \mathrm{SiCl}$ (1.5 equiv) THF $-78{ }^{\circ} \mathrm{C}$ to rt, $4 \mathrm{~h}$

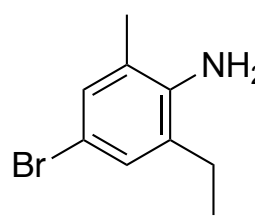

S1 (96\%)

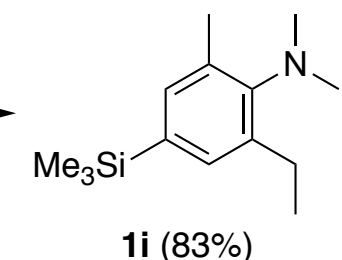

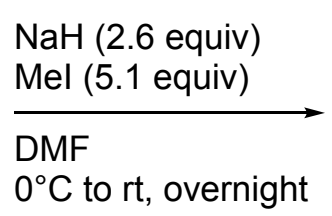<smiles>CCc1cc(Br)cc(C)c1N(C)C</smiles>

S2 (78\%) 
The brominaiton of 2-ethyl-6-methylaniline was carried out according to the procedure reported. $^{3}$ A $500 \mathrm{~mL}$ three-neck flask, equipped with a magnetic stirring bar, a condenser, a rubber septum, and a three-way stopcock, was charged with EtOAc $(80 \mathrm{~mL})$, DMSO $(1.65 \mathrm{~g}$, $21.1 \mathrm{mmol}$ ), 2-ethyl-6-methylaniline (2.71 g, $20.0 \mathrm{mmol}$ ), and hydrogen bromide (48\% aqueous solution, $7.68 \mathrm{~g}, 45.6 \mathrm{mmol}$ ). The mixture was stirred for $1 \mathrm{~h}$ at $60^{\circ} \mathrm{C}$. After cooling to room temperature, water $(100 \mathrm{~mL})$ was added to the flask, and potassium carbonate was added until the aqueous layer was basic. The organic materials were extracted with hexane $(100 \mathrm{~mL} \times 3)$, washed with brine (200 $\mathrm{mL} \times 1)$, and dried over anhydrous magnesium sulfate. The crude product was purified by Kugelrhor distillation. S1 (4.12 g, $19.2 \mathrm{mmol}, 96 \%$ yield) was obtained as a colorless oil. S1: ${ }^{1} \mathrm{H}$ NMR (400 MHz, $\left.\mathrm{CDCl}_{3}\right) \delta 7.071$ (s, 1H), 7.069 (s, 1H), 3.60 (broad s, 2H, NH$H_{2}$ ), 2.49 (q, $J=7.6 \mathrm{~Hz}, 2 \mathrm{H}), 2.16(\mathrm{~s}, 3 \mathrm{H}), 1.25(\mathrm{t}, J=7.6 \mathrm{~Hz}, 3 \mathrm{H}) \cdot{ }^{13} \mathrm{C} \mathrm{NMR}\left(101 \mathrm{MHz}, \mathrm{CDCl}_{3}\right) \delta 141.3$, $130.4,129.3,128.5,123.9,109.8,24.0,17.5,12.8$. HRMS (APCI, positive) $\mathrm{m} / z$ calcd for $\mathrm{C}_{9} \mathrm{H}_{13} \mathrm{BrN}^{+}[\mathrm{M}+\mathrm{H}]^{+}:$214.0226, found: 214.0228.

According to the procedure given for 1a, S2 (2.82 $\mathrm{g}, 11.6 \mathrm{mmol}, 78 \%$ yield) was prepared as a colorless oil from $\mathbf{S 1}(3.16 \mathrm{~g}, 14.8 \mathrm{mmol}) . \mathbf{S 2}:{ }^{1} \mathrm{H}$ NMR $\left(400 \mathrm{MHz}, \mathrm{CDCl}_{3}\right) \delta 7.15[\mathrm{~d}(\mathrm{AB}$ pattern), $J=2.4 \mathrm{~Hz}, 1 \mathrm{H}$ ], 7.12 [d (AB pattern), $J=2.4 \mathrm{~Hz}, 1 \mathrm{H}$ ], 2.79 (s, 6H), 2.63 (q, $J=7.6 \mathrm{~Hz}$, 2H), $2.26(\mathrm{~s}, 3 \mathrm{H}), 1.19$ (t, $J=7.6 \mathrm{~Hz}, 3 \mathrm{H}) .{ }^{13} \mathrm{C} \mathrm{NMR}\left(101 \mathrm{MHz}, \mathrm{CDCl}_{3}\right) \delta 148.5,146.0,139.9$, 131.6, 129.8, 118.4, 42.9, 25.0, 19.1, 15.4. HRMS (APCI, positive) $m / z$ calcd for $\mathrm{C}_{11} \mathrm{H}_{17} \mathrm{BrN}^{+}[\mathrm{M}$ $+\mathrm{H}^{+}: 242.0539$, found: 242.0541 .

An oven dried $50 \mathrm{~mL}$ two-neck flask, equipped with a magnetic stirring bar, a rubber septum, and a three-way stopcock, was evacuated and backfilled with nitrogen. THF $(8 \mathrm{~mL})$ and $\mathbf{S 2}$ ( $0.454 \mathrm{~g}, 1.87 \mathrm{mmol})$ were added to the flask, and the mixture was cooled to $-78{ }^{\circ} \mathrm{C}$ by dry ice/acetone bath. $s$-BuLi (1.0 M in cyclohexane, $2.4 \mathrm{~mL}, 2.4 \mathrm{mmol})$ was added slowly to the flask, and the resulting mixture was stirred for $2 \mathrm{~h}$ at $-78^{\circ} \mathrm{C} . \mathrm{Me}_{3} \mathrm{SiCl}(0.311 \mathrm{~g}, 2.86 \mathrm{mmol})$ was added slowly to the flask. The dry ice/acetone bath was removed, and the mixture was stirred for $4 \mathrm{~h}$ at room temperature. Water $(20 \mathrm{~mL})$ was added to the flask. The organic materials were extracted with $\mathrm{Et}_{2} \mathrm{O}(20 \mathrm{~mL} \times 3)$, washed with brine (50 $\left.\mathrm{mL} \times 1\right)$, and dried over anhydrous magnesium sulfate. The crude product was purified by column chromatography on silica gel (SiliaFlash; eluent: hexane only) and Kugelrhor distillation. 1i ( $0.367 \mathrm{~g}, 1.56 \mathrm{mmol}, 83 \%$ yield) was obtained 
as a colorless oil. 1i: ${ }^{1} \mathrm{H}$ NMR (400 MHz, $\left.\mathrm{CDCl}_{3}\right) \delta 7.21(\mathrm{~s}, 1 \mathrm{H}), 7.16(\mathrm{~s}, 1 \mathrm{H}), 2.85(\mathrm{~s}, 6 \mathrm{H}), 2.70$ (q, $J=7.6 \mathrm{~Hz}, 2 \mathrm{H}), 2.34$ (s, 3H), 1.25 (t, $J=7.6 \mathrm{~Hz}, 3 \mathrm{H}), 0.28(\mathrm{~s}, 9 \mathrm{H}) .{ }^{13} \mathrm{C}$ NMR $(101 \mathrm{MHz}$, $\left.\mathrm{CDCl}_{3}\right) \delta 150.1,142.8,136.7,136.6,134.4,132.4,43.1,25.4,19.4,15.9,-0.8$. HRMS (APCI, positive) $\mathrm{m} / z$ calcd for $\mathrm{C}_{14} \mathrm{H}_{26} \mathrm{NSi}^{+}[\mathrm{M}+\mathrm{H}]^{+}: 236.1829$, found: 236.1830 .

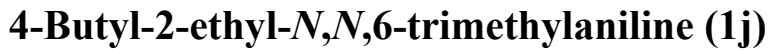

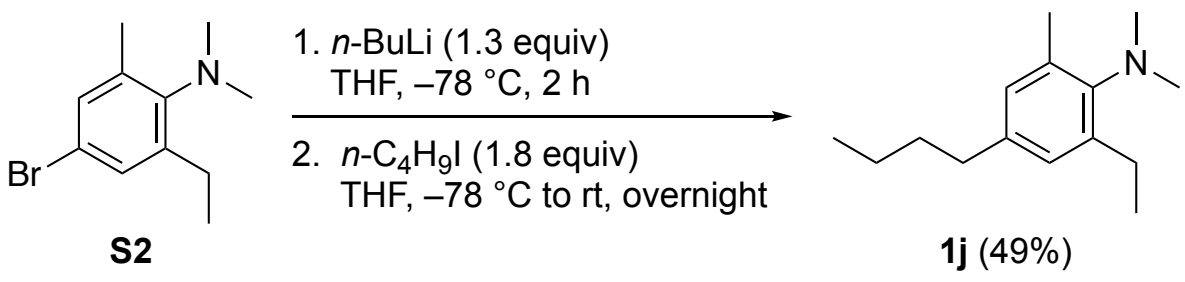

An oven dried $100 \mathrm{~mL}$ two-neck flask, equipped with a magnetic stirring bar, a rubber septum, and a three-way stopcock, was evacuated and backfilled with nitrogen. THF ( $8 \mathrm{~mL})$ and $\mathbf{S 2}$ ( $0.440 \mathrm{~g}, 1.82 \mathrm{mmol})$ were added to the flask, and the mixture was cooled to $-78{ }^{\circ} \mathrm{C}$ by dry ice/acetone bath. $n$-BuLi (1.6 M solution in hexane, $1.5 \mathrm{~mL}, 2.4 \mathrm{mmol}$ ) was added slowly to the flask, and the resulting mixture was stirred for $2 \mathrm{~h}$ at $-78{ }^{\circ} \mathrm{C}$. Butyl iodide $(0.599 \mathrm{~g}, 3.26 \mathrm{mmol})$ was added slowly to the flask. The dry ice/acetone bath was removed, and the mixture was stirred overnight at room temperature. Water $(20 \mathrm{~mL})$ was added to the flask. The organic materials were extracted with $\mathrm{Et}_{2} \mathrm{O}(20 \mathrm{~mL} \times 3)$, washed with brine (50 $\left.\mathrm{mL} \times 1\right)$, and dried over anhydrous magnesium sulfate. The crude product was purified by column chromatography on silica gel (SiliaFlash; eluent: hexane:Et $\mathrm{t}_{2} \mathrm{O}=$ 99:1), GPC, and Kugelrhor distillation. 1j (0.196 g, 0.893 mmol, $49 \%$ yield) was obtained as a colorless oil. $1 \mathbf{j}:{ }^{1} \mathrm{H}$ NMR $\left(400 \mathrm{MHz}, \mathrm{CDCl}_{3}\right) \delta 6.86(\mathrm{~s}, 1 \mathrm{H})$, $6.82(\mathrm{~s}, 1 \mathrm{H}), 2.83$ (s, 6H), 2.66 (q, $J=7.6 \mathrm{~Hz}, 2 \mathrm{H}), 2.50-2.56(\mathrm{~m}, 2 \mathrm{H}), 2.29$ (s, 3H), 1.55-1.65 (m, 2H), 1.34-1.46 (m, 2H), 1.22 (t, $J=7.6 \mathrm{~Hz}, 3 \mathrm{H}), 0.96$ (t, $J=7.6 \mathrm{~Hz}, 3 \mathrm{H}) .{ }^{13} \mathrm{C}$ NMR (101 MHz, $\left.\mathrm{CDCl}_{3}\right) \delta 146.9,143.5,139.7,137.3,129.0,127.0,43.2,35.3,33.9,25.2,22.8,19.3,15.8,14.2$. HRMS (APCI, positive) $m / z$ calcd for $\mathrm{C}_{15} \mathrm{H}_{26} \mathrm{~N}^{+}[\mathrm{M}+\mathrm{H}]^{+}: 220.2060$, found: 220.2061 . 


\section{2,6-Diethyl-4-methoxy- $N, N$-dimethylaniline (1k)}

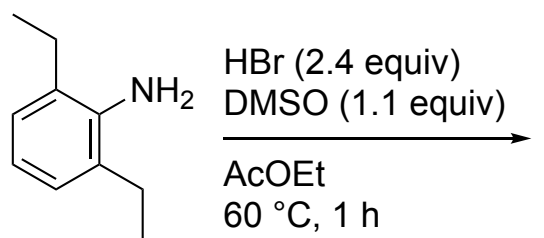

$\mathrm{MeOH}$ (4.0 equiv)

Cul $(9 \mathrm{~mol} \%)$

3,4,7,8-tetramethylphenanthroline (15 mol \%)

$\mathrm{CsCO}_{3}$ (2.0 equiv)

toluene

$110^{\circ} \mathrm{C}, 48 \mathrm{~h}$

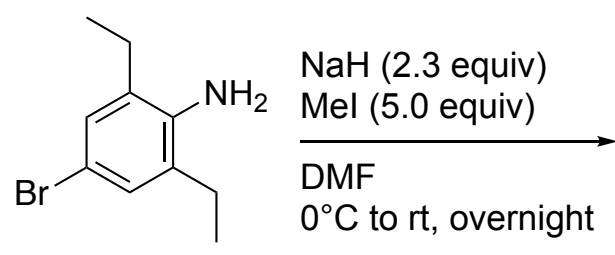

S3 (98\%)

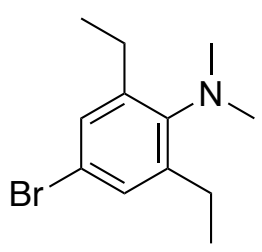

S4 $(91 \%)$

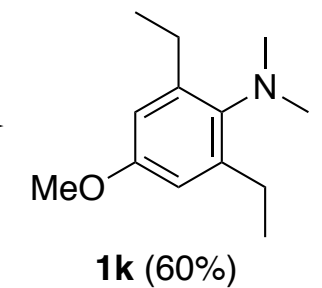

According to the procedure given for S1, S3 (4.39 g, $19.2 \mathrm{mmol}, 98 \%$ yield) was prepared as a colorless oil from 2,6-diethylaniline (2.92 g, $19.6 \mathrm{mmol})$. S3: ${ }^{1} \mathrm{H}$ NMR (400 MHz, $\left.\mathrm{CDCl}_{3}\right) \delta 7.08$ $(\mathrm{s}, 2 \mathrm{H}), 3.63($ broad s, 2H, NH$), 2.50(\mathrm{q}, J=7.6 \mathrm{~Hz}, 4 \mathrm{H}), 1.25(\mathrm{t}, J=7.6 \mathrm{~Hz}, 6 \mathrm{H}) .{ }^{13} \mathrm{C} \mathrm{NMR}$ $\left(101 \mathrm{MHz}, \mathrm{CDCl}_{3}\right) \delta 140.7,129.7,128.5,110.3,24.1,12.8$. HRMS (APCI, positive) $\mathrm{m} / z$ calcd for $\mathrm{C}_{10} \mathrm{H}_{15} \mathrm{BrN}^{+}[\mathrm{M}+\mathrm{H}]^{+}:$228.0382, found: 228.0385 .

According to the procedure given for 1a, S4 (3.84 g, $15.0 \mathrm{mmol}, 91 \%$ yield) was prepared as a colorless oil from S3 (3.74 mg, $16.4 \mathrm{mmol})$. S4: ${ }^{1} \mathrm{H}$ NMR (400 MHz, $\left.\mathrm{CDCl}_{3}\right) \delta 7.16(\mathrm{~s}, 2 \mathrm{H}), 2.80$ $(\mathrm{s}, 6 \mathrm{H}), 2.63(\mathrm{q}, J=7.6 \mathrm{~Hz}, 4 \mathrm{H}), 1.21(\mathrm{t}, J=7.6 \mathrm{~Hz}, 6 \mathrm{H}) .{ }^{13} \mathrm{C} \mathrm{NMR}\left(101 \mathrm{MHz}, \mathrm{CDCl}_{3}\right) \delta 148.0$, $146.4,129.9,119.1,43.6,24.9,15.4$. HRMS (APCI, positive) $m / z$ calcd for $\mathrm{C}_{12} \mathrm{H}_{19} \mathrm{BrN}^{+}[\mathrm{M}+$ $\mathrm{H}]^{+}: 256.0695$, found: 256.0696 .

The copper-catalyzed $\mathrm{C}-\mathrm{O}$ coupling was carried out according to the procedure reported. ${ }^{4} \mathrm{~A}$ glass tube (outside diameter: $20 \mathrm{~mm}$ ) having PTFE stopcock (J. Young), equipped with a magnetic stirring bar, was charged with $\mathrm{CuI}(35.4 \mathrm{mg}, 0.186 \mathrm{mmol})$, 3,4,7,8-tetramethyl-1,10-phenanthroline (73.7 mg, $0.312 \mathrm{mmol}$ ), and $\mathrm{CsCO}_{3}$ (1.36 g, $4.17 \mathrm{mmol}$ ). The tube was evacuated and backfilled with argon. S4 (0.528 g, $2.06 \mathrm{mmol}), \mathrm{MeOH}$ (13.9 mg, $0.012 \mathrm{mmol})$, and toluene $(1 \mathrm{~mL})$ were added to the tube, and it was sealed by the stopcock. The mixture was stirred at $110{ }^{\circ} \mathrm{C}$ by a heating magnetic stirrer with an aluminum heating block (hole size: $21 \mathrm{~mm}$ diameter $\times 33 \mathrm{~mm}$ depth). After $48 \mathrm{~h}$, the tube was cooled to room temperature, and water $(20 \mathrm{~mL})$ was added to the tube. The organic materials were extracted with AcOEt $(20 \mathrm{~mL} x$ 3), washed with brine (50 mL x 1), and dried over anhydrous magnesium sulfate. The crude product was purified by column chromatography on silica gel (SiliaFlash; eluent: hexane:AcOEt 
$=100: 0$ to $0: 100)$ and Kugelrhor distillation. $1 \mathbf{k}(0.257 \mathrm{~g}, 1.24 \mathrm{mmol}, 60 \%$ yield $)$ was obtained as a colorless oil. 1k: ${ }^{1} \mathrm{H}$ NMR $\left(400 \mathrm{MHz}, \mathrm{CDCl}_{3}\right) \delta 6.61(\mathrm{~s}, 2 \mathrm{H}), 3.80(\mathrm{~s}, 3 \mathrm{H}), 2.83(\mathrm{~s}, 6 \mathrm{H}), 2.66(\mathrm{q}$, $J=7.6 \mathrm{~Hz}, 4 \mathrm{H}), 1.24(\mathrm{t}, J=7.6 \mathrm{~Hz}, 6 \mathrm{H}) .{ }^{13} \mathrm{C} \mathrm{NMR}\left(101 \mathrm{MHz}, \mathrm{CDCl}_{3}\right) \delta 157.1,145.6,142.1$, 112.0, 55.3, 44.0, 25.3, 15.6. HRMS (APCI, positive) $\mathrm{m} / z$ calcd for $\mathrm{C}_{13} \mathrm{H}_{22} \mathrm{NO}^{+}[\mathrm{M}+\mathrm{H}]^{+}$: 208.1696, found: 208.1696.

\section{2,6-Diethyl- $N, N, N^{\prime}, N^{\prime}$-tetramethylbenzene-1,4-diamine (11)}

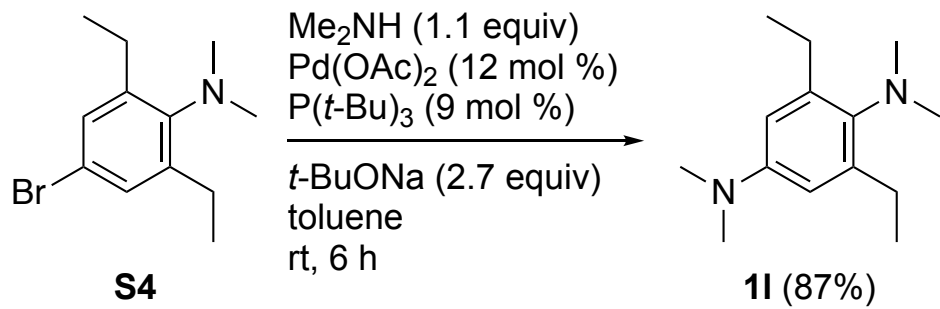

The palladium-catalyzed $\mathrm{C}-\mathrm{N}$ coupling was carried out according to the procedure reported. ${ }^{5}$ In a glovebox, an oven dried $100 \mathrm{~mL}$ two-neck flask, equipped with a magnetic stirring bar, a three-way stop cock, and a rubber septum, was charged with $\operatorname{Pd}(\mathrm{OAc})_{2}(38 \mathrm{mg}, 0.169 \mathrm{mmol})$, $\mathrm{P}(t-\mathrm{Bu})_{3}(26 \mathrm{mg}, 0.129 \mathrm{mmol}), t-\mathrm{BuONa}(0.37 \mathrm{~g}, 3.85 \mathrm{mmol})$, and toluene $(2 \mathrm{~mL})$. The stopcock was closed, and the flask was taken out from glovebox. Dimethylamine $(0.75 \mathrm{~mL}, 1.5 \mathrm{mmol})$ and S4 $(0.362 \mathrm{~g}, 1.41 \mathrm{mmol})$ were added, and the mixture was stirred for $6 \mathrm{~h}$ at room temperature. Water $(20 \mathrm{~mL})$ was added to the flask. The organic materials were extracted with $\mathrm{Et}_{2} \mathrm{O}(20 \mathrm{~mL} \mathrm{x}$ 3), washed with brine (50 mL x 1), and dried over anhydrous magnesium sulfate. The crude product was purified by column chromatography on silica gel (SiliaFlash; eluent: hexane: $\mathrm{Et}_{2} \mathrm{O}=$ 20:1) and Kugelrhor distillation. 11 (0.272 g, $1.23 \mathrm{mmol}, 87 \%$ yield) was obtained as a colorless oil. 11: ${ }^{1} \mathrm{H}$ NMR (400 MHz, $\left.\mathrm{CDCl}_{3}\right) \delta 6.47(\mathrm{~s}, 2 \mathrm{H}), 2.94(\mathrm{~s}, 6 \mathrm{H}), 2.83(\mathrm{~s}, 6 \mathrm{H}), 2.65$ (q, $J=7.6 \mathrm{~Hz}$, 4H), $1.25(\mathrm{t}, J=7.6 \mathrm{~Hz}, 6 \mathrm{H}) .{ }^{13} \mathrm{C} \mathrm{NMR}\left(101 \mathrm{MHz}, \mathrm{CDCl}_{3}\right) \delta 148.6,144.9,139.1,111.7,44.2$, 41.1, 25.6, 16.0. HRMS (APCI, positive) $m / z$ calcd for $\mathrm{C}_{14} \mathrm{H}_{25} \mathrm{~N}_{2}{ }^{+}[\mathrm{M}+\mathrm{H}]^{+}: 221.2012$, found: 221.2013 


\section{3-Chloro-2,6-diethyl- $N, N$-dimethylaniline (1m)}

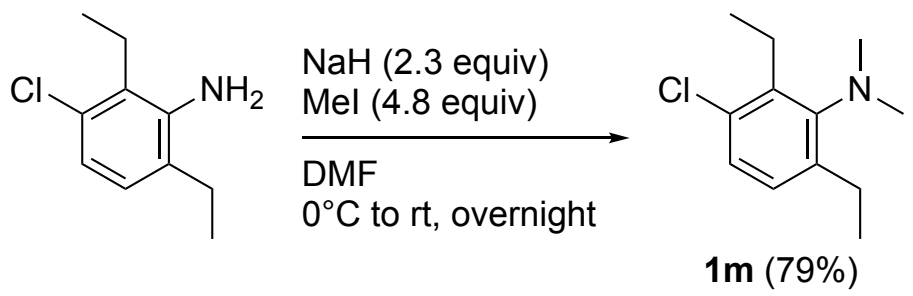

According to the procedure given for 1a, 1m (1.76 $\mathrm{g}, 8.31 \mathrm{mmol}, 79 \%$ yield) was prepared as a yellow oil from 3-chloro-2,6-diethylaniline (Aldrich, $1.92 \mathrm{~g}, 10.5 \mathrm{mmol}) .1 \mathrm{~m}:{ }^{1} \mathrm{H}$ NMR (400 $\left.\mathrm{MHz}, \mathrm{CDCl}_{3}\right) \delta 7.14(\mathrm{~d}, J=8.4 \mathrm{~Hz}, 1 \mathrm{H}), 6.97(\mathrm{~d}, J=8.4 \mathrm{~Hz}, 1 \mathrm{H}), 2.85(\mathrm{~s}, 6 \mathrm{H}), 2.82(\mathrm{q}, J=7.6$ $\mathrm{Hz}, 2 \mathrm{H}), 2.63(\mathrm{q}, J=7.6 \mathrm{~Hz}, 2 \mathrm{H}), 1.22(\mathrm{t}, J=7.6 \mathrm{~Hz}, 3 \mathrm{H}), 1.21(\mathrm{t}, J=7.6 \mathrm{~Hz}, 3 \mathrm{H}) .{ }^{13} \mathrm{C} \mathrm{NMR}$ $\left(101 \mathrm{MHz}, \mathrm{CDCl}_{3}\right) \delta 150.5,143.3,142.6,132.1,127.7,127.0,43.9,24.8,23.2,15.5,14.2$. HRMS (APCI, positive) $m / z$ calcd for $\mathrm{C}_{12} \mathrm{H}_{19} \mathrm{ClN}^{+}[\mathrm{M}+\mathrm{H}]^{+}: 212.1201$, found: 212.1202 .

\section{2,6-Diethyl-3-(phthalimidomethyl)- $N, N$-dimethylaniline (1n)}

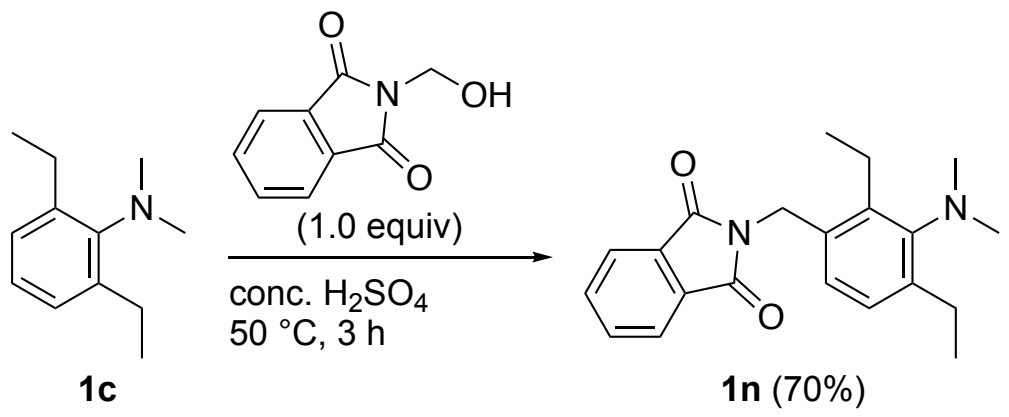

The titled compound was synthesized according to the procedure reported. ${ }^{6}$ An oven dried $200 \mathrm{~mL}$ three-neck flask, equipped with a magnetic stirring bar, a rubber septum, and a three-way stopcock, was evacuated and backfilled with nitrogen. 1c $(1.75 \mathrm{~g}, 9.87 \mathrm{mmol})$ and conc. $\mathrm{H}_{2} \mathrm{SO}_{4}$ $(10 \mathrm{~mL})$ was added to the flask. $N$-hydroxymethylphthalimide (Kanto, $1.75 \mathrm{~g}, 9.88 \mathrm{mmol}$ ) was added to the flask over $20 \mathrm{~min}$. The mixture was stirred for $3 \mathrm{~h}$ at $50{ }^{\circ} \mathrm{C}$. After cooling to room temperature, ice was added to the flask, and potassium carbonate was added until the aqueous layer was basic. The organic materials were extracted with $\mathrm{Et}_{2} \mathrm{O}(50 \mathrm{~mL}$ x 3), washed with brine (50 mL x 1), and dried over anhydrous magnesium sulfate. The crude product was purified by distillation using Kugelrohr. 1n (2.33 g, $6.93 \mathrm{mmol}, 70 \%$ yield) was as a white solid. 1n: ${ }^{1} \mathrm{H}$ NMR $\left(400 \mathrm{MHz}, \mathrm{CDCl}_{3}\right) \delta$ 7.83-7.89 (m, 2H), 7.69-7.75 (m, 2H), 7.03 [d (AB pattern), $J=8.0$ $\mathrm{Hz}, 1 \mathrm{H}$ ], 6.97 [d (AB pattern), $J=8.0 \mathrm{~Hz}, 1 \mathrm{H}], 4.89$ (s, 2H), 2.92 (q, $J=7.6 \mathrm{~Hz}, 2 \mathrm{H}), 2.84$ (s, 
$6 \mathrm{H}), 2.61(\mathrm{q}, J=7.6 \mathrm{~Hz}, 2 \mathrm{H}), 1.23(\mathrm{t}, J=7.6 \mathrm{~Hz}, 3 \mathrm{H}), 1.19(\mathrm{t}, J=7.6 \mathrm{~Hz}, 3 \mathrm{H}) .{ }^{13} \mathrm{C} \mathrm{NMR}(101$ $\left.\mathrm{MHz} \mathrm{CDCl}_{3}\right) \delta 168.4,149.3,143.9,142.9,134.1,132.4,132.3,127.1,125.5,123.4,44.0,39.1$, 24.7, 21.5, 15.7, 15.4. HRMS (APCI, positive) $m / z$ calcd for $\mathrm{C}_{21} \mathrm{H}_{25} \mathrm{~N}_{2} \mathrm{O}_{2}{ }^{+}[\mathrm{M}+\mathrm{H}]^{+}: 337.1911$, found: 337.1912 .

\section{4,4'-Methylenebis(2-ethyl- $N, N$-dimethylaniline) (10)}

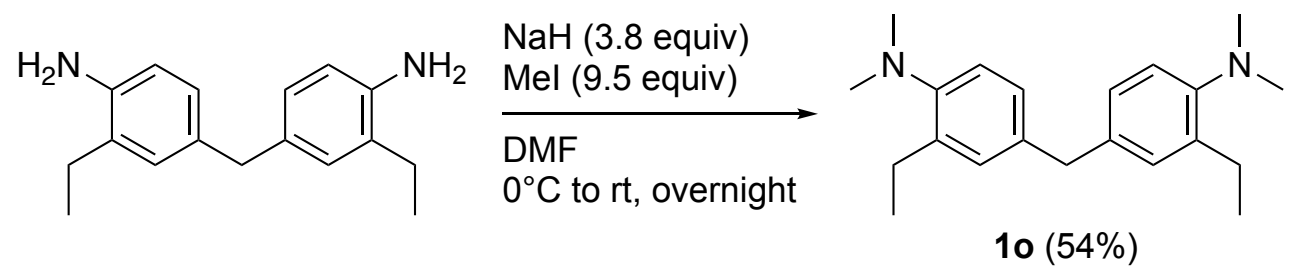

According to the procedure given for 1a, 10 (1.84 g, $5.92 \mathrm{mmol}, 54 \%$ yield) was prepared as a colorless oil from 4,4'-methylenebis(2-ethylaniline) (Combi Blocks, $2.81 \mathrm{~g}, 11.0 \mathrm{mmol}$ ). 1o: ${ }^{1} \mathrm{H}$ NMR $\left(400 \mathrm{MHz}, \mathrm{CDCl}_{3}\right) \delta 7.24(\mathrm{~d}, J=2.0,2 \mathrm{H}), 7.17(\mathrm{~d}, J=8.4 \mathrm{~Hz}, 2 \mathrm{H}), 7.13(\mathrm{dd}, J=8.4,2.0$ $\mathrm{Hz}, 2 \mathrm{H}), 4.03(\mathrm{~s}, 2 \mathrm{H}), 2.87(\mathrm{q}, J=7.6 \mathrm{~Hz}, 4 \mathrm{H}), 2.81(\mathrm{~s}, 12 \mathrm{H}), 1.40(\mathrm{t}, J=7.6 \mathrm{~Hz}, 6 \mathrm{H}) .{ }^{13} \mathrm{C} \mathrm{NMR}$ $\left(101 \mathrm{MHz}, \mathrm{CDCl}_{3}\right) \delta 150.6,138.8,136.4,129.6,126.7,119.3,45.4,41.1,23.6,15.1 . \mathrm{HRMS}$ (APCI, positive) $m / z$ calcd for $\mathrm{C}_{21} \mathrm{H}_{31} \mathrm{~N}_{2}^{+}[\mathrm{M}+\mathrm{H}]^{+}: 311.2482$, found: 311.2485 .

\section{4,4'-Methylenebis(2-ethyl- $N, N, 6$-trimethylaniline) (1p)}
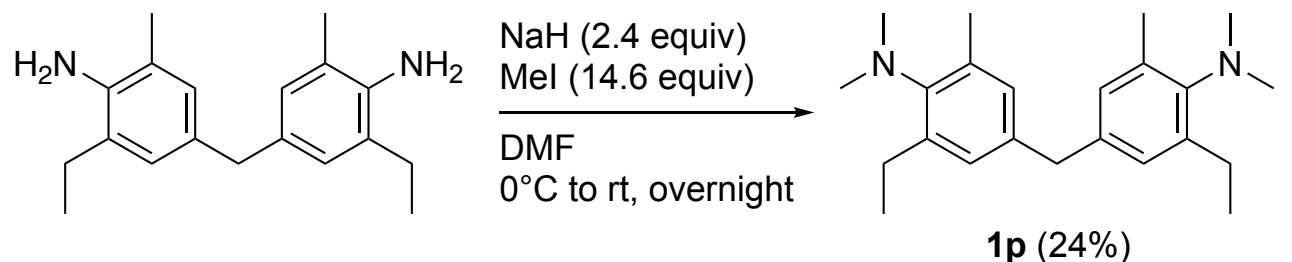

According to the procedure given for 1a, 1p (0.843 g, $2.49 \mathrm{mmol}, 24 \%$ yield $)$ was prepared as a white solid from 4,4'-methylenebis(2-ethyl-6-methylaniline) (TCI, $2.97 \mathrm{~g}, 10.5 \mathrm{mmol}) .1 \mathbf{1 p :}{ }^{1} \mathrm{H}$ NMR $\left(400 \mathrm{MHz}, \mathrm{CDCl}_{3}\right) \delta 6.94(\mathrm{~d}, J=2.0 \mathrm{~Hz}, 2 \mathrm{H}), 6.86(\mathrm{~d}, J=2.0 \mathrm{~Hz}, 2 \mathrm{H}), 3.83(\mathrm{~s}, 2 \mathrm{H}), 2.85$ (s, 12H), 2.69 (q, $J=7.6 \mathrm{~Hz}, 4 \mathrm{H}), 2.31(\mathrm{~s}, 6 \mathrm{H}), 1.24(\mathrm{t}, J=7.6 \mathrm{~Hz}, 6 \mathrm{H}) .{ }^{13} \mathrm{C} \mathrm{NMR}(101 \mathrm{MHz}$, $\left.\mathrm{CDCl}_{3}\right) \delta 147.2,143.6,138.1,137.5,129.5,127.5,43.2,41.2,25.3,19.4,15.8$. HRMS (APCI, positive) $m / z$ calcd for $\mathrm{C}_{23} \mathrm{H}_{35} \mathrm{~N}_{2}^{+}[\mathrm{M}+\mathrm{H}]^{+}: 339.2795$, found: 339.2799 . 


\section{4,4'-Methylenebis(2,6-diethyl- $N, N$-dimethylaniline) (1q)}

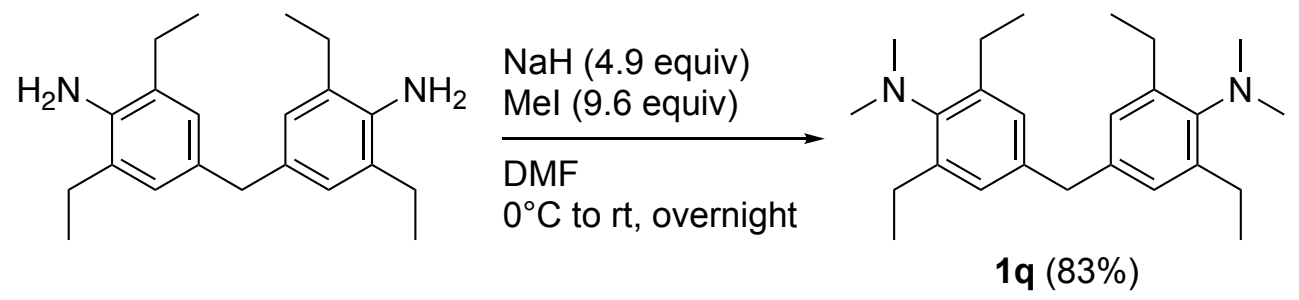

According to the procedure given for 1a, 1q $(3.14 \mathrm{~g}, 8.57 \mathrm{mmol}, 83 \%$ yield $)$ was prepared as a white solid from 4,4'-methylenebis(2,6-diethylaniline) (TCI, $3.20 \mathrm{~g}, 10.3 \mathrm{mmol}$ ). 1q: ${ }^{1} \mathrm{H}$ NMR $\left(400 \mathrm{MHz}, \mathrm{CDCl}_{3}\right) \delta 6.93(\mathrm{~s}, 4 \mathrm{H}), 3.88(\mathrm{~s}, 2 \mathrm{H}), 2.86(\mathrm{~s}, 12 \mathrm{H}), 2.67(\mathrm{q}, J=7.6 \mathrm{~Hz}, 8 \mathrm{H}), 1.24(\mathrm{t}, J$ $=7.6 \mathrm{~Hz}, 12 \mathrm{H}) .{ }^{13} \mathrm{C}$ NMR $\left(101 \mathrm{MHz}, \mathrm{CDCl}_{3}\right) \delta 146.8,144.0,138.3,127.6,43.9,41.4,25.1,15.8$. HRMS (APCI, positive) $m / z$ calcd for $\mathrm{C}_{25} \mathrm{H}_{39} \mathrm{~N}_{2}{ }^{+}[\mathrm{M}+\mathrm{H}]^{+}: 367.3108$, found: 367.3111 .

\section{$N$-Benzyl-2-ethyl- $N$-methylaniline (4)}<smiles>CCc1ccccc1Br</smiles>

(1.1 equiv)<smiles>CNCc1ccccc1</smiles>

$\mathrm{HN}$
$\mathrm{Pd}(\mathrm{dba})_{2}(4 \mathrm{~mol} \%)$

$\mathrm{P}(t-\mathrm{Bu})_{3}(4 \mathrm{~mol} \%)$

$t$-BuONa ( 1.5 equiv)

toluene

$60^{\circ} \mathrm{C}$, overnight

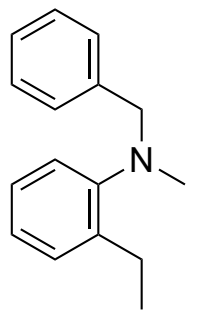

$4(29 \%)$

According to the procedure given for 11, 4 (0.168 g, $0.746 \mathrm{mmol}, 29 \%$ yield) was prepared as a colorless oil from 1-bromo-2-ethylbenzene $(0.502 \mathrm{~g}, 2.71 \mathrm{mmol})$ and $N$-methylbenzylamine (0.309 g, $2.55 \mathrm{mmol})$ using $\mathrm{Pd}(\mathrm{dba})_{2}(4 \mathrm{~mol} \%)$ instead of $\mathrm{Pd}(\mathrm{OAc})_{2} .4:{ }^{1} \mathrm{H} \mathrm{NMR}(400 \mathrm{MHz}$, $\left.\mathrm{CDCl}_{3}\right) \delta$ 7.42-7.47 (m, 2H), 7.35-7.42 (m, 2H), 7.28-7.34 (m, 2H), 7.18-7.27 (m, 2H), 7.09-7.15 $(\mathrm{m}, 1 \mathrm{H}), 4.06(\mathrm{~s}, 2 \mathrm{H}), 2.89$ (q, $J=7.6 \mathrm{~Hz}, 2 \mathrm{H}), 2.62(\mathrm{~s}, 3 \mathrm{H}), 1.34(\mathrm{t}, J=7.6 \mathrm{~Hz}, 3 \mathrm{H}) .{ }^{13} \mathrm{C} \mathrm{NMR}$ $\left(101 \mathrm{MHz}, \mathrm{CDCl}_{3}\right) \delta 152.3,139.6,139.4,129.1,128.5,128.4,127.1,126.5,124.0,121.0,61.9$, 41.9, 23.8, 15.1. HRMS (APCI, positive) $m / z$ calcd for $\mathrm{C}_{16} \mathrm{H}_{20} \mathrm{~N}^{+}[\mathrm{M}+\mathrm{H}]^{+}: 226.1590$, found: 226.1590 . 


\section{2-Ethyl- $N$-methyl- $N$-phenylaniline (6)}
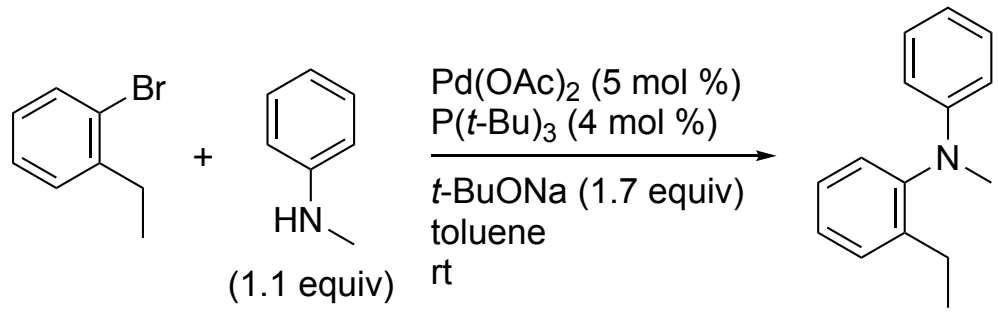

$6(44 \%)$

According to the procedure given for $11,6(0.230 \mathrm{~g}, 1.02 \mathrm{mmol}, 44 \%$ yield $)$ was prepared as a yellow oil from 1-bromo-2-ethylbenzene $(0.428 \mathrm{~g}, 2.31 \mathrm{mmol})$ and $N$-methylaniline $(0.263 \mathrm{~g}$, 2.45 mmol). 6: ${ }^{1} \mathrm{H}$ NMR (400 MHz, $\left.\mathrm{CDCl}_{3}\right) \delta$ 7.36-7.42 (m, 1H), 7.27-7.33 (m, 2H), 7.14-7.25 $(\mathrm{m}, 3 \mathrm{H}), 6.75(\mathrm{t}, J=7.2 \mathrm{~Hz}, 1 \mathrm{H}), 6.58(\mathrm{~d}, J=8.0 \mathrm{~Hz}, 2 \mathrm{H}), 3.27$ (s, 3H), 2.59 (q, $J=7.6 \mathrm{~Hz}, 2 \mathrm{H})$, $1.21(\mathrm{t}, J=7.6 \mathrm{~Hz}, 3 \mathrm{H}) .{ }^{13} \mathrm{C} \mathrm{NMR}\left(101 \mathrm{MHz}, \mathrm{CDCl}_{3}\right) \delta 149.7,146.6,143.0,129.7,129.0,128.7$, 127.7, 126.9, 116.8, 112.9, 39.7, 24.2, 14.8. HRMS (APCI, positive) $m / z$ calcd for $\mathrm{C}_{15} \mathrm{H}_{18} \mathrm{~N}^{+}[\mathrm{M}$ $+\mathrm{H}]^{+}: 212.1434$, found: 212.1433 .

\section{$N, N, 2-T r i m e t h y l a n i l i n e ~(8)$}

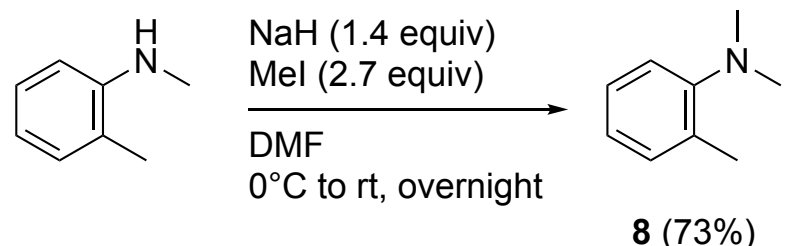

According to the procedure given for 1a, $8(1.01 \mathrm{~g}, 7.47 \mathrm{mmol}, 73 \%$ yield $)$ was prepared as a colorless oil from N,2-dimethylylaniline (TCI, $1.24 \mathrm{~g}, 10.2 \mathrm{mmol}$ ). 8: ${ }^{1} \mathrm{H}$ NMR (400 MHz, $\left.\mathrm{CDCl}_{3}\right) \delta 7.17-7.23(\mathrm{~m}, 2 \mathrm{H}), 7.08(\mathrm{dd}, J=8.4,1.2 \mathrm{~Hz}, 1 \mathrm{H}), 6.99(\mathrm{td}, J=7.2,1.2 \mathrm{~Hz}, 1 \mathrm{H}), 2.74(\mathrm{~s}$, $6 \mathrm{H}), 2.38(\mathrm{~s}, 3 \mathrm{H}) .{ }^{13} \mathrm{C} \mathrm{NMR}\left(101 \mathrm{MHz}, \mathrm{CDCl}_{3}\right) \delta 152.8,132.2,131.3,126.5,122.7,118.5,44.3$, 18.5. HRMS (APCI, positive) $m / z$ calcd for $\mathrm{C}_{9} \mathrm{H}_{14} \mathrm{~N}^{+}[\mathrm{M}+\mathrm{H}]^{+}: 136.1121$, found: 136.1120 .

\section{$N, N$-Dimethyl-2-vinylaniline (3a)}

This compound was prepared from 2-bromo- $N, N$-dimethylaniline by the method reported. ${ }^{7}$ 


\section{$N, N$-Dibenzyl-2-ethylaniline (9)}
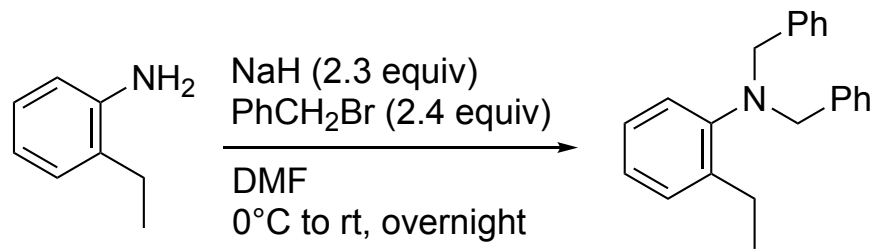

$9(16 \%)$

An oven dried $200 \mathrm{~mL}$ three-neck flask, equipped with a magnetic stirring bar, a rubber septum, and a three-way stopcock, was charged with $\mathrm{NaH}$ (ca. 60\% dispersion in mineral oil, 0.9 g, $23 \mathrm{mmol})$. The flask was evacuated and backfilled with nitrogen. DMF (20 mL) and 2-ethylaniline (Wako, $1.19 \mathrm{~g}, 9.82 \mathrm{mmol}$ ) were added to the flask, and the mixture was cooled to $0{ }^{\circ} \mathrm{C}$ by ice/water bath. Benzyl bromide $(4.04 \mathrm{~g}, 23.6 \mathrm{mmol})$ was added slowly to the flask. The ice/water bath was removed, and the mixture was stirred overnight at room temperature. Hexane $(30 \mathrm{~mL})$, AcOEt $(30 \mathrm{~mL})$, and water $(60 \mathrm{~mL})$ were added to the flask. The organic materials were washed with water $(60 \mathrm{~mL} \times 4)$ and brine $(50 \mathrm{~mL} \times 1)$, and dried over anhydrous magnesium sulfate. The crude product was purified by column chromatography on silica gel (SiliaFlash; eluent: hexane: $\left.\mathrm{Et}_{2} \mathrm{O}=97: 3\right)$ and Kugelrhor distillation. 9 (0.474 g, $1.57 \mathrm{mmol}, 16 \%$ yield $)$ was

obtained as a colorless oil. 9: ${ }^{1} \mathrm{H}$ NMR $\left(400 \mathrm{MHz}, \mathrm{CDCl}_{3}\right) \delta$ 7.34-7.47 (m, 11H), 7.17-7.28 (m, $3 \mathrm{H}), 4.24(\mathrm{~s}, 4 \mathrm{H}), 3.06(\mathrm{q}, J=7.6 \mathrm{~Hz}, 2 \mathrm{H}), 1.43(\mathrm{t}, J=7.6 \mathrm{~Hz}, 3 \mathrm{H}) .{ }^{13} \mathrm{C} \mathrm{NMR}\left(101 \mathrm{MHz}, \mathrm{CDCl}_{3}\right)$ $\delta 149.5,140.3,138.6,129.01,128.97,128.2,127.0,126.0,124.3,123.2,57.9,23.5,14.9$. HRMS (APCI, positive) $m / z$ calcd for $\mathrm{C}_{22} \mathrm{H}_{24} \mathrm{~N}^{+}[\mathrm{M}+\mathrm{H}]^{+}: 302.1903$, found: 302.1905 .

\section{N,3-Dimethylindoline (11a)}

This compound was prepared from 3-methylindole by the method reported. ${ }^{7}$

\section{Reaction Conditions (Table 1)}

General procedure: In a glovebox, a glass tube (outside diameter: $20 \mathrm{~mm}$, internal volume: $16 \mathrm{~mL}$ ) having PTFE stopcock (J. Young), equipped with a magnetic stirring bar, was charged with $\left[\mathrm{IrCl}\left(\mathrm{C}_{2} \mathrm{H}_{4}\right)_{2}\right]_{2}(0.0030 \mathrm{mmol})$, a ligand $(0.0060 \mathrm{mmol}), \mathbf{1 a}(0.10 \mathrm{mmol})$, additive $(0-0.30$ mmol), and mesitylene $(0.2 \mathrm{~mL})$. The tube was sealed by the stopcock and was taken out from the glovebox (Figure S1). The mixture was stirred at $110-150{ }^{\circ} \mathrm{C}$ by a heating magnetic stirrer 
with an aluminum heating block (hole size: $21 \mathrm{~mm}$ diameter x $33 \mathrm{~mm}$ depth). After $24 \mathrm{~h}$, the tube was cooled to room temperature. Diphenylmethane (TCI, $16.8 \mathrm{mg}, 0.10 \mathrm{mmol}$, internal standard) was added, and the resulting mixture was analyzed by ${ }^{1} \mathrm{H}$ NMR to determine the yield of $2 \mathbf{a}$.

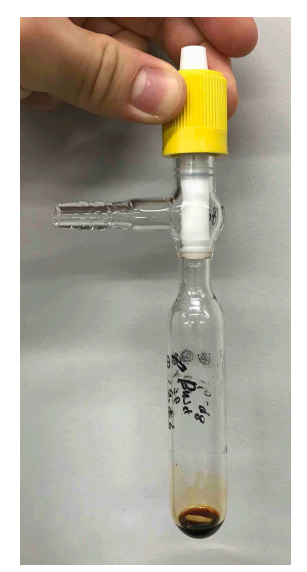

Figure S1. A Glass Tube (internal volume: $16 \mathrm{~mL}$ ) Containing a Reaction Mixture

\section{The reaction of 1a to afford 1,3-dimethyl- $1 H$-indole (2a) (entry 4, Table 1)}

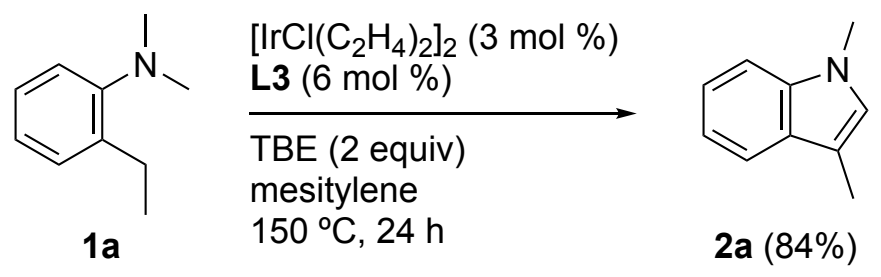

In a glovebox, a glass tube (outside diameter: $20 \mathrm{~mm}$, internal volume: $16 \mathrm{~mL}$ ) having PTFE stopcock (J. Young), equipped with a magnetic stirring bar, was charged with $\left[\operatorname{IrCl}\left(\mathrm{C}_{2} \mathrm{H}_{4}\right)_{2}\right]_{2}$ (3.42 mg, $0.00603 \mathrm{mmol}), \mathbf{L 3}(13.9 \mathrm{mg}, 0.0118 \mathrm{mmol}), 1 \mathrm{a}(29.9 \mathrm{mg}, 0.200 \mathrm{mmol})$, tert-butylethylene $(37.6 \mathrm{mg}, 0.447 \mathrm{mmol})$, and mesitylene $(0.4 \mathrm{~mL})$. The tube was sealed by the stopcock and was taken out from the glovebox. The mixture was stirred for $24 \mathrm{~h}$ at $150{ }^{\circ} \mathrm{C}$ by a heating magnetic stirrer with an aluminum heating block (hole size: $21 \mathrm{~mm}$ diameter x $33 \mathrm{~mm}$ depth). 2a (24.4 mg, $0.168 \mathrm{mmol}, 84 \%$ ) was obtained as a colorless oil after purification by column chromatography on silica gel (eluent: hexane:Et $\left.\mathrm{Et}_{2} \mathrm{O}: \mathrm{Et}_{3} \mathrm{~N}=45: 1: 1\right)$. 2a: ${ }^{1} \mathrm{H}$ NMR (400 $\left.\mathrm{MHz}, \mathrm{CDCl}_{3}\right) \delta 7.61(\mathrm{dt}, J=7.6,1.2 \mathrm{~Hz}, 1 \mathrm{H}), 7.31$ [dt (AB pattern), $J=8.0,1.2 \mathrm{~Hz}, 1 \mathrm{H}$ ], 7.22-7.28 (m, 1H), 7.14 (ddd, $J=8.0,6.8,1.2 \mathrm{~Hz}, 1 \mathrm{H}), 6.85$ (d, $J=1.2 \mathrm{~Hz}, 1 \mathrm{H}), 3.75$ (s, 3H), $2.36(\mathrm{~d}, J=1.2 \mathrm{~Hz}, 3 \mathrm{H}) .{ }^{13} \mathrm{C} \mathrm{NMR}\left(101 \mathrm{MHz}, \mathrm{CDCl}_{3}\right) \delta 137.1,128.7,126.6,121.5,119.0,118.6$, 110.2, 109.1, 32.6, 9.7. HRMS (APCI, positive) $m / z$ calcd for $\mathrm{C}_{10} \mathrm{H}_{12} \mathrm{~N}^{+}[\mathrm{M}+\mathrm{H}]^{+}: 146.0964$, found: 146.0963 . 


\section{Additional Results in Screening of Reaction Conditions}

The reaction of $\mathbf{1 a}$ proceeded efficiently to afford $\mathbf{2 a}$ in good yields when $\left[\operatorname{IrCl}\left(\mathrm{C}_{2} \mathrm{H}_{4}\right)_{2}\right]_{2}$, $\left[\operatorname{IrCl}(\mathrm{coe})_{2}\right]_{2},[\operatorname{IrCl}(\mathrm{cod})]_{2}$ were used as catalyst precursors (entries 1-3, Table S1). Addition of $\operatorname{AgOTf}(6 \mathrm{~mol} \%)$ inhibited the reaction (entry 4). No reaction took place using $\left[\mathrm{RhCl}\left(\mathrm{C}_{2} \mathrm{H}_{4}\right)_{2}\right]_{2}$ as a catalyst precursor (entry 5).

Table S1. Screening of Catalyst Precursors ${ }^{a}$

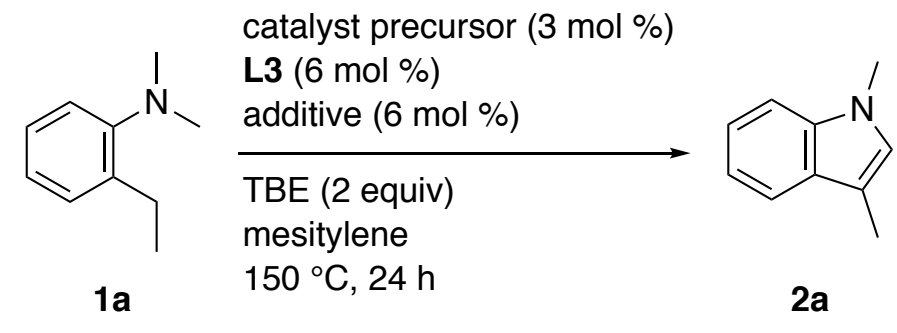

\begin{tabular}{cllc}
\hline entry & catalyst precursor & additive & $\%$ yield of $\mathbf{2 a}^{b}$ \\
\hline 1 & {$\left[\operatorname{IrCl}\left(\mathrm{C}_{2} \mathrm{H}_{4}\right)_{2}\right]_{2}$} & - & $93(84)^{c}$ \\
2 & {$\left[\operatorname{IrCl}(\mathrm{Coe})_{2}\right]_{2}$} & - & 76 \\
3 & {$[\operatorname{IrCl}(\mathrm{cod})]_{2}$} & - & 85 \\
4 & {$\left[\operatorname{IrCl}\left(\mathrm{C}_{2} \mathrm{H}_{4}\right)_{2}\right]_{2}$} & AgOTf & 0 \\
5 & {$\left[\mathrm{RhCl}\left(\mathrm{C}_{2} \mathrm{H}_{4}\right)_{2}\right]_{2}$} & - & no reaction \\
\hline
\end{tabular}

a 1a $(0.10 \mathrm{mmol})$, a catalyst precursor (3 $\mathrm{mol} \%)$, L3 $(6 \mathrm{~mol}$ $\%$ ), additive (0 or $6 \mathrm{~mol} \%$ ), TBE (2 equiv), and mesitylene $(0.2 \mathrm{~mL})$ were stirred at $150{ }^{\circ} \mathrm{C}$ for $24 \mathrm{~h}$. ${ }^{b}$ Determined by ${ }^{1} \mathrm{H}$ NMR. ${ }^{c}$ Isolated yield in the $0.2 \mathrm{mmol}$ scale reaction.

1,3-Bis[(di-tert-butylphosphaneyl)methyl]benzene and 1,3-bis[(di-tert-butylphosphaneyl)oxy]benzene, which have been known as ligands to form PCP-pincer iridium complexes, were applied to the reaction of $\mathbf{1 a}$. No reaction took place.

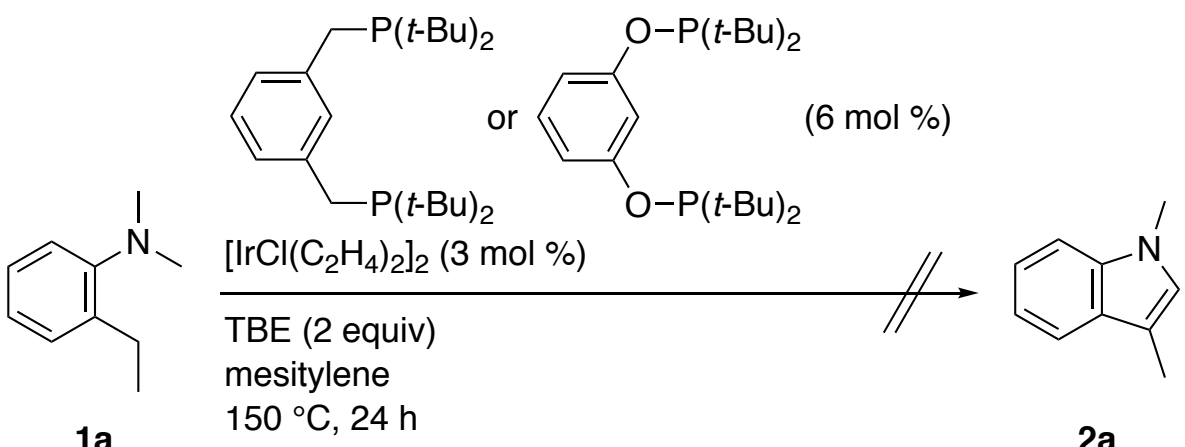

$1 a$ $150^{\circ} \mathrm{C}, 24 \mathrm{~h}$

no reation 


\section{Iridium-Catalyzed Dehydrogenation-Intramolecular Dehydrogenative $\mathbf{C}-\mathrm{H} / \mathrm{C}-\mathrm{H}$ Coupling of 1 (Scheme 2)}

General procedure A: In a glovebox, a glass tube (outside diameter: $20 \mathrm{~mm}$, internal volume: $16 \mathrm{~mL}$ ) having PTFE stopcock (J. Young), equipped with a magnetic stirring bar, was charged with $\left[\mathrm{IrCl}\left(\mathrm{C}_{2} \mathrm{H}_{4}\right)_{2}\right]_{2}(3.41 \mathrm{mg}, 0.00600 \mathrm{mmol}), \mathbf{L 3}(14.2 \mathrm{mg}, 0.0120 \mathrm{mmol}), 1$ (0.200 mmol), tert-butylethylene $(33.7 \mathrm{mg}, 0.400 \mathrm{mmol})$, and mesitylene $(0.4 \mathrm{~mL})$. The tube was sealed by the stopcock and was taken out from the glovebox (Figure S1). The mixture was stirred for $24 \mathrm{~h}$ at $150{ }^{\circ} \mathrm{C}$ by a heating magnetic stirrer with an aluminum heating block (hole size: $21 \mathrm{~mm}$ diameter x $33 \mathrm{~mm}$ depth). The product 2 was purified by column chromatography on silica gel.

General procedure B (for the reaction that afford a mixture of alkyl- and alkenyl-substituted indoles): ${ }^{8}$ In a glovebox, a glass tube (outside diameter: $20 \mathrm{~mm}$, internal volume: $16 \mathrm{~mL}$ ) having PTFE stopcock (J. Young), equipped with a magnetic stirring bar, was charged with $\left[\operatorname{IrCl}\left(\mathrm{C}_{2} \mathrm{H}_{4}\right)_{2}\right]_{2}(3.41 \mathrm{mg}, 0.00600 \mathrm{mmol}), \mathbf{L 3}(14.2 \mathrm{mg}, 0.0120 \mathrm{mmol}), 1$ (0.200 mmol), tert-butylethylene $(33.7 \mathrm{mg}, 0.400 \mathrm{mmol})$, and mesitylene $(0.4 \mathrm{~mL})$. The tube was sealed by the stopcock and was taken out from the glovebox (Figure S1). The mixture was stirred at $150{ }^{\circ} \mathrm{C}$ by a heating magnetic stirrer with an aluminum heating block (hole size: $21 \mathrm{~mm}$ diameter x $33 \mathrm{~mm}$ depth). After $24 \mathrm{~h}$, the tube was cooled to room temperature, and it was brought in a glovebox. THF $(0.4 \mathrm{~mL})$ was added to the tube. The tube was sealed by the stopcock again and was taken out from the glovebox. The mixture was stirred at $135^{\circ} \mathrm{C}$ by a heating magnetic stirrer. After $3 \mathrm{~h}$, the tube was cooled to room temperature, and the volatiles were removed under reduced pressure. The product 2 was purified by column chromatography on silica gel.

\section{The Reaction of 4 to Afford 1-Benzyl-3-methyl-1H-indole (5) (entry 1)}
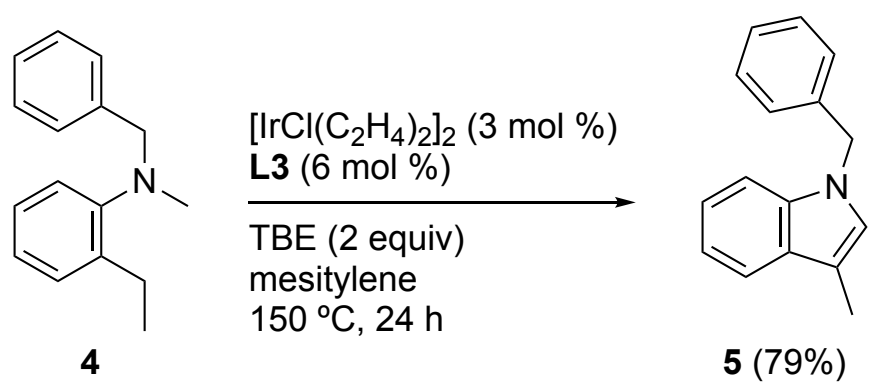
According to the general procedure $A$, the reaction was carried out at $150{ }^{\circ} \mathrm{C}$ for $24 \mathrm{~h}$ using $\left[\operatorname{IrCl}\left(\mathrm{C}_{2} \mathrm{H}_{4}\right)_{2}\right]_{2}(3.64 \mathrm{mg}, 0.00641 \mathrm{mmol}), \mathbf{L 3}(14.1 \mathrm{mg}, 0.0120 \mathrm{mmol}), 4$ (44.6 mg, $\left.0.198 \mathrm{mmol}\right)$, tert-butylethylene $(33.6 \mathrm{mg}, 0.399 \mathrm{mmol})$, and mesitylene $(0.4 \mathrm{~mL}) .5(34.5 \mathrm{mg}, 0.156 \mathrm{mmol}$, $79 \%$ ) was obtained as a white solid after purification by column chromatography on silica gel (eluent: hexane: $\left.\mathrm{Et}_{2} \mathrm{O}: \mathrm{Et}_{3} \mathrm{~N}=30: 1: 1\right)$. 5: ${ }^{1} \mathrm{H} \mathrm{NMR}\left(400 \mathrm{MHz}, \mathrm{CDCl}_{3}\right) \delta$ 7.62-7.66 (m, 1H), 7.26-7.36 (m, 4H), 7.13-7.24 (m, 4H), 6.93 (d, J=1.2 Hz, 1H), 5.29 (s, 2H), 2.39 (d, $J=1.2 \mathrm{~Hz}$, 3H). ${ }^{13} \mathrm{C} \mathrm{NMR}\left(101 \mathrm{MHz}, \mathrm{CDCl}_{3}\right) \delta 138.0,136.8,129.0,128.8,127.6,126.9,126.0,121.7,119.2$, 118.9, 111.0, 109.6, 49.9, 9.8. HRMS (APCI, positive) $m / z$ calcd for $\mathrm{C}_{16} \mathrm{H}_{16} \mathrm{~N}^{+}[\mathrm{M}+\mathrm{H}]^{+}$: 222.1277, found: 222.1276 .

\section{The Reaction of 6 to Afford 1-Phenyl-3-methyl-1H-indole (7) (entry 2)}

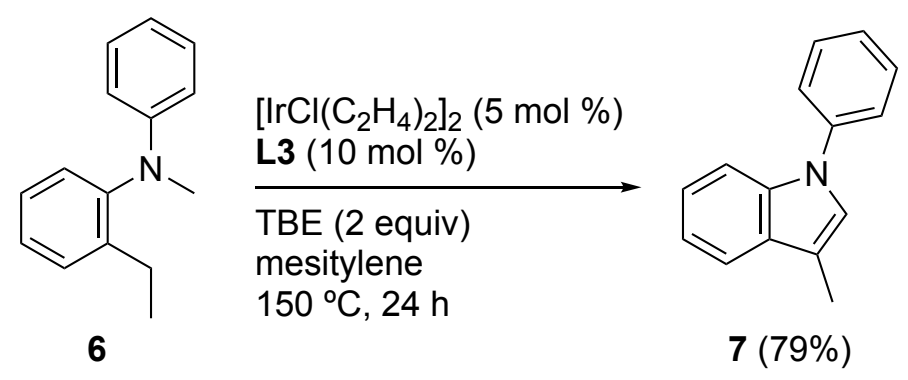

According to the general procedure $A$, the reaction was carried out at $150^{\circ} \mathrm{C}$ for $24 \mathrm{~h}$ using $\left[\operatorname{IrCl}\left(\mathrm{C}_{2} \mathrm{H}_{4}\right)_{2}\right]_{2}(5.65 \mathrm{mg}, 0.00995 \mathrm{mmol}), \mathbf{L 3}(24.2 \mathrm{mg}, 0.0205 \mathrm{mmol}), 6$ (41.5 mg, $\left.0.196 \mathrm{mmol}\right)$, tert-butylethylene $(34.0 \mathrm{mg}, 0.404 \mathrm{mmol})$, and mesitylene $(0.4 \mathrm{~mL}) .7$ (32.1 mg, $0.155 \mathrm{mmol}$, $79 \%$ ) was obtained as a green oil after purification by column chromatography on silica gel (eluent: hexane: $\left.\mathrm{Et}_{2} \mathrm{O}: \mathrm{Et}_{3} \mathrm{~N}=45: 1: 1\right) .7:{ }^{1} \mathrm{H} \mathrm{NMR}\left(400 \mathrm{MHz}, \mathrm{CDCl}_{3}\right) \delta 7.66-7.70(\mathrm{~m} 1 \mathrm{H})$, 7.59-7.62 (m, 1H), 7.50-7.56 (m, 4H), 7.32-7.38 (m, 1H), 7.19-7.30 (m, 2H), 7.18 (d, J=1.2 Hz, $1 \mathrm{H}), 2.44(\mathrm{~d}, J=1.2 \mathrm{~Hz}, 3 \mathrm{H}) .{ }^{13} \mathrm{C} \mathrm{NMR}\left(101 \mathrm{MHz}, \mathrm{CDCl}_{3}\right) \delta 140.1,136.1,129.9,129.7,126.0$, 125.6, 124.1, 122.5, 119.9, 119.3, 112.9, 110.5, 9.7. HRMS (APCI, positive) $\mathrm{m} / z$ calcd for $\mathrm{C}_{15} \mathrm{H}_{14} \mathrm{~N}^{+}[\mathrm{M}+\mathrm{H}]^{+}:$208.1121, found: 208.1121 . 
The Reaction of $1 \mathrm{~b}$ to Afford 1,3,7-Trimethyl-1H-indole (2b) (entry 3)

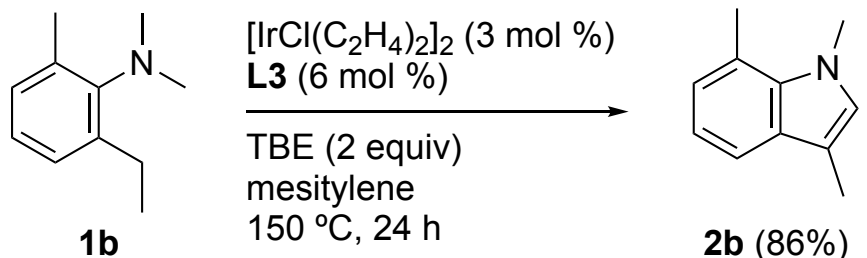

According to the general procedure $A$, the reaction was carried out at $150{ }^{\circ} \mathrm{C}$ for $24 \mathrm{~h}$ using $\left[\operatorname{IrCl}\left(\mathrm{C}_{2} \mathrm{H}_{4}\right)_{2}\right]_{2}(3.22 \mathrm{mg}, 0.00567 \mathrm{mmol}), \mathbf{L 3}(14.0 \mathrm{mg}, 0.0119 \mathrm{mmol}), \mathbf{1 b}(34.0 \mathrm{mg}, 0.208 \mathrm{mmol})$, tert-butylethylene (33.9 mg, $0.403 \mathrm{mmol})$, and mesitylene $(0.4 \mathrm{~mL}) .2 \mathbf{b}(28.5 \mathrm{mg}, 0.179 \mathrm{mmol}$, $86 \%$ including $<2 \%$ of a structural isomer) was obtained as a white solid after purification by column chromatography on silica gel (eluent: hexane: $\left.\mathrm{Et}_{2} \mathrm{O}: \mathrm{Et}_{3} \mathrm{~N}=45: 1: 1\right) .2 \mathbf{b}$ : ${ }^{1} \mathrm{H} \mathrm{NMR}(400$ $\left.\mathrm{MHz}, \mathrm{CDCl}_{3}\right) \delta 7.41-7.46(\mathrm{~m}, 1 \mathrm{H}), 7.02$ (t, $\left.J=7.2 \mathrm{~Hz}, 1 \mathrm{H}\right), 6.94$ [d (AB pattern), $J=7.2 \mathrm{~Hz}, 1 \mathrm{H}$ ], $6.74(\mathrm{~d}, J=1.2 \mathrm{~Hz}, 1 \mathrm{H}), 4.02(\mathrm{~s}, 3 \mathrm{H}), 2.79(\mathrm{~s}, 3 \mathrm{H}), 2.33(\mathrm{~d}, J=1.2 \mathrm{~Hz}, 3 \mathrm{H}) .{ }^{13} \mathrm{C}$ NMR $(101 \mathrm{MHz}$, $\left.\mathrm{CDCl}_{3}\right) \delta 135.8,129.8,128.3,124.2,121.2,118.9,117.2,109.9,36.4,19.8,9.6$. HRMS (APCI, positive) $m / z$ calcd for $\mathrm{C}_{11} \mathrm{H}_{14} \mathrm{~N}^{+}[\mathrm{M}+\mathrm{H}]^{+}: 160.1121$, found: 160.1119 .

\section{The Reaction of 1c to Afford 7-Ethyl-1,3-dimethyl-1H-indole (2c) (entry 4)}

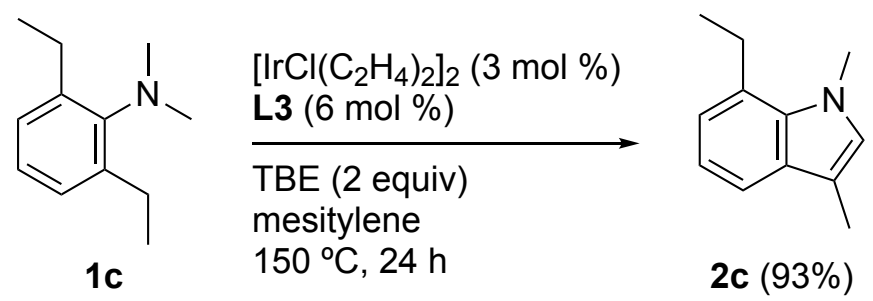

According to the general procedure $A$, the reaction was carried out at $150^{\circ} \mathrm{C}$ for $24 \mathrm{~h}$ using $\left[\operatorname{IrCl}\left(\mathrm{C}_{2} \mathrm{H}_{4}\right)_{2}\right]_{2}(3.72 \mathrm{mg}, 0.00655 \mathrm{mmol}), \mathbf{L 3}(14.6 \mathrm{mg}, 0.0124 \mathrm{mmol}), 1 \mathrm{c}(36.1 \mathrm{mg}, 0.204 \mathrm{mmol})$, tert-butylethylene (35.3 mg, $0.419 \mathrm{mmol})$, and mesitylene $(0.4 \mathrm{~mL}) .2 \mathrm{c}(32.7 \mathrm{mg}, 0.189 \mathrm{mmol}$, 93\%) was obtained as a white solid after purification by column chromatography on silica gel (eluent: hexane: $\left.\mathrm{Et}_{2} \mathrm{O}: \mathrm{Et}_{3} \mathrm{~N}=45: 1: 1\right) .2 \mathrm{c}:{ }^{1} \mathrm{H} \mathrm{NMR}\left(400 \mathrm{MHz}, \mathrm{CDCl}_{3}\right) \delta 7.45(\mathrm{dd}, J=8.0,1.2 \mathrm{~Hz}$, 1H), $7.08(\mathrm{t}, J=8.0 \mathrm{~Hz}, 1 \mathrm{H}), 7.00-7.04(\mathrm{~m}, 1 \mathrm{H}), 6.75(\mathrm{~d}, J=1.2 \mathrm{~Hz}, 1 \mathrm{H}), 4.00(\mathrm{~s}, 3 \mathrm{H}), 3.14$ (q, $J$ $=7.6 \mathrm{~Hz}, 2 \mathrm{H}), 2.34(\mathrm{~d}, J=1.2 \mathrm{~Hz}, 3 \mathrm{H}), 1.39(\mathrm{t}, J=7.6 \mathrm{~Hz}, 3 \mathrm{H}) .{ }^{13} \mathrm{C} \mathrm{NMR}\left(101 \mathrm{MHz}, \mathrm{CDCl}_{3}\right) \delta$ 135.0, 130.3, 128.6, 127.8, 122.5, 119.1, 117.1, 110.1, 36.4, 25.6, 16.9, 9.6. HRMS (APCI, positive) $m / z$ calcd for $\mathrm{C}_{12} \mathrm{H}_{16} \mathrm{~N}^{+}[\mathrm{M}+\mathrm{H}]^{+}:$174.1277, found: 174.1276 .

\section{1 mmol scale reaction}

In a glovebox, a glass tube (internal volume: $36 \mathrm{~mL}$ ) having PTFE stopcock (J. Young), 
equipped with a magnetic stirring bar, was charged with $\left[\operatorname{IrCl}\left(\mathrm{C}_{2} \mathrm{H}_{4}\right)_{2}\right]_{2}(17.2 \mathrm{mg}, 0.0303 \mathrm{mmol})$, L3 (71.4 mg, $0.0605 \mathrm{mmol})$, 1c (177 mg, $0.998 \mathrm{mmol})$, tert-butylethylene (172 mg, $2.04 \mathrm{mmol}$ ), and mesitylene $(2 \mathrm{~mL})$. The tube was sealed by the stopcock and was taken out from the glovebox. The mixture was stirred at $150{ }^{\circ} \mathrm{C}$ by an oil bath. After $24 \mathrm{~h}$, the tube was cooled to room temperature, and the volatiles were removed under reduced pressure. $2 \mathbf{c}(145 \mathrm{mg}, 0.837$ mmol, 84\%) was obtained as a colorless oil after purification by column chromatography on silica gel (eluent: hexane: $\mathrm{Et}_{2} \mathrm{O}: \mathrm{Et}_{3} \mathrm{~N}=45: 1: 1$ ).

\section{The Reaction of 1d to Afford 3-Ethyl-1-methyl-1H-indole (2d) (entry 5)}

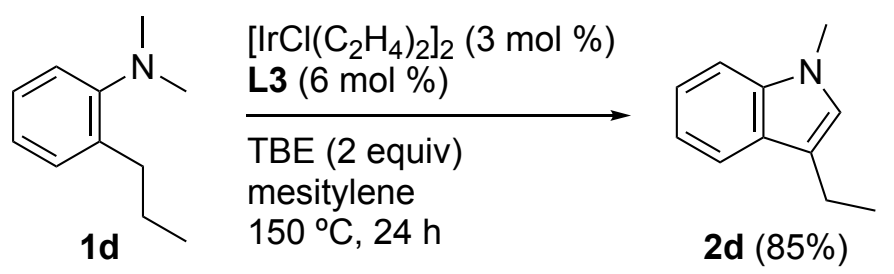

According to the general procedure $A$, the reaction was carried out at $150{ }^{\circ} \mathrm{C}$ for $24 \mathrm{~h}$ using $\left[\mathrm{IrCl}\left(\mathrm{C}_{2} \mathrm{H}_{4}\right)_{2}\right]_{2}(3.83 \mathrm{mg}, 0.00675 \mathrm{mmol}), \mathbf{L 3}(14.8 \mathrm{mg}, 0.0126 \mathrm{mmol}), \mathbf{1 d}(33.7 \mathrm{mg}, 0.206 \mathrm{mmol})$, tert-butylethylene $(33.7 \mathrm{mg}, 0.400 \mathrm{mmol})$, and mesitylene $(0.4 \mathrm{~mL}) .2 \mathrm{~d}(27.9 \mathrm{mg}, 0.175 \mathrm{mmol}$, $85 \%$ ) was obtained as a colorless oil after purification by column chromatography on silica gel (eluent: hexane: $\left.\mathrm{Et}_{2} \mathrm{O}: \mathrm{Et}_{3} \mathrm{~N}=47: 1: 1\right) . \mathbf{2 d}:{ }^{1} \mathrm{H} \mathrm{NMR}\left(400 \mathrm{MHz}, \mathrm{CDCl}_{3}\right) \delta 7.65(\mathrm{dt}, J=8.0,1.2 \mathrm{~Hz}$, 1H), 7.32 [dt (AB pattern), $J=8.0,1.2 \mathrm{~Hz}, 1 \mathrm{H}], 7.23-7.29$ (m, 1H), 7.14 (ddd, $J=7.6,6.8,1.2$ $\mathrm{Hz}, 1 \mathrm{H}), 6.86$ (s, 1H), 3.77 (s, 3H), 2.83 (q, $J=7.6 \mathrm{~Hz}, 2 \mathrm{H}), 1.37$ (t, $J=7.6 \mathrm{~Hz}, 3 \mathrm{H}) .{ }^{13} \mathrm{C} \mathrm{NMR}$ $\left(101 \mathrm{MHz}, \mathrm{CDCl}_{3}\right) \delta 137.2,127.9,125.5,121.6,119.2,118.6,117.4,109.2,32.6,18.4,14.9$. HRMS (APCI, positive) $m / z$ calcd for $\mathrm{C}_{11} \mathrm{H}_{14} \mathrm{~N}^{+}[\mathrm{M}+\mathrm{H}]^{+}: 160.1121$, found: 160.1119 . 
The Reaction of 1e to Afford 1-Methyl-3-propyl-1H-indole (2e) (entry 6)
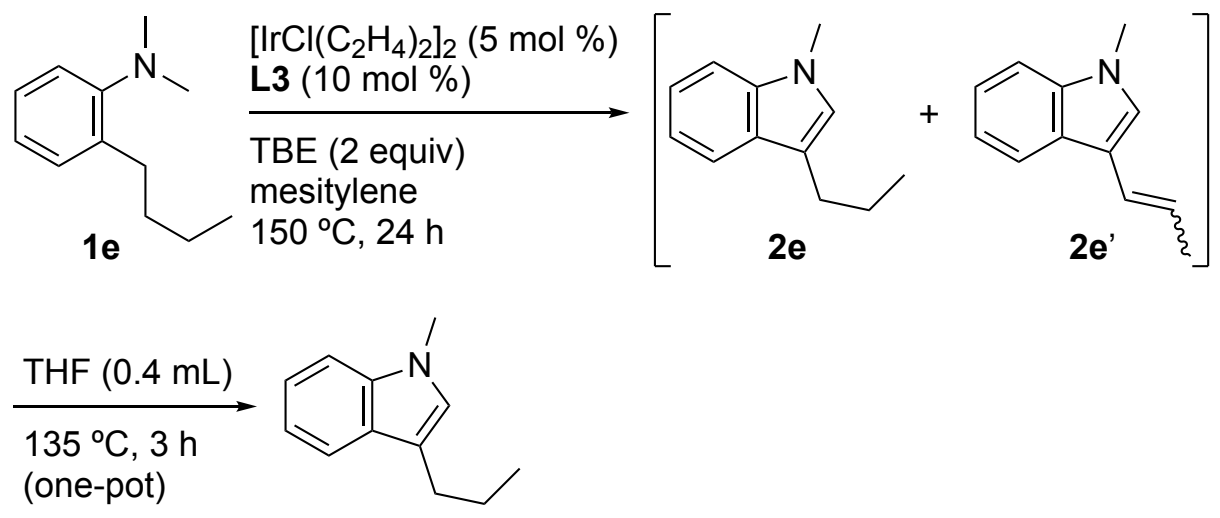

2e $(60 \%)$

According to the general procedure $B$, the reaction was carried out at $150{ }^{\circ} \mathrm{C}$ for $24 \mathrm{~h}$ using $\left[\operatorname{IrCl}\left(\mathrm{C}_{2} \mathrm{H}_{4}\right)_{2}\right]_{2}(5.33 \mathrm{mg}, 0.00939 \mathrm{mmol}), \mathbf{L 3}(23.0 \mathrm{mg}, 0.0195 \mathrm{mmol}), 1 \mathrm{e}(30.5 \mathrm{mg}, 0.172 \mathrm{mmol})$, tert-butylethylene $(31.3 \mathrm{mg}, 0.372 \mathrm{mmol})$, and mesitylene $(0.4 \mathrm{~mL})$. The reaction mixture was then treated with THF $(0.4 \mathrm{~mL})$ for $3 \mathrm{~h}$ at $135^{\circ} \mathrm{C} .2 \mathrm{e}(18.0 \mathrm{mg}, 0.104 \mathrm{mmol}, 60 \%)$ was obtained as a colorless oil after purification by column chromatography on silica gel (eluent: hexane: $\left.\mathrm{Et}_{2} \mathrm{O}: \mathrm{Et}_{3} \mathrm{~N}=40: 1: 1\right)$. 2e: ${ }^{1} \mathrm{H} \mathrm{NMR}\left(400 \mathrm{MHz}, \mathrm{CDCl}_{3}\right) \delta 7.62(\mathrm{dt}, J=8.0,1.2 \mathrm{~Hz}, 1 \mathrm{H})$, 7.30 [dt (AB pattern), $J=8.0,1.2 \mathrm{~Hz}, 1 \mathrm{H}], 7.23$ (ddd, $J=8.0,6.8,1.2 \mathrm{~Hz}, 1 \mathrm{H}), 7.11$ (ddd, $J=8.0$, 6.8, 1.2 Hz, 1H), $6.84(\mathrm{~s}, 1 \mathrm{H}), 3.75(\mathrm{~s}, 3 \mathrm{H}), 2.74$ (t, $J=7.6 \mathrm{~Hz}, 2 \mathrm{H}), 1.74$ (sextet, $J=7.6 \mathrm{~Hz}, 2 \mathrm{H})$, $1.02(\mathrm{t}, J=7.6 \mathrm{~Hz}, 3 \mathrm{H}) .{ }^{13} \mathrm{C} \mathrm{NMR}\left(101 \mathrm{MHz}, \mathrm{CDCl}_{3}\right) \delta 137.1,128.1,126.2,121.5,119.2,118.5$, 115.6, 109.2, 32.7, 27.4, 23.7, 14.3. HRMS (APCI, positive) $m / z$ calcd for $\mathrm{C}_{12} \mathrm{H}_{16} \mathrm{~N}^{+}[\mathrm{M}+\mathrm{H}]^{+}$: 174.1277, found: 174.1277 .

\section{The Reaction of 1 f to Afford 1,3,5-Trimethyl-1H-indole (2f) (entry 7)}

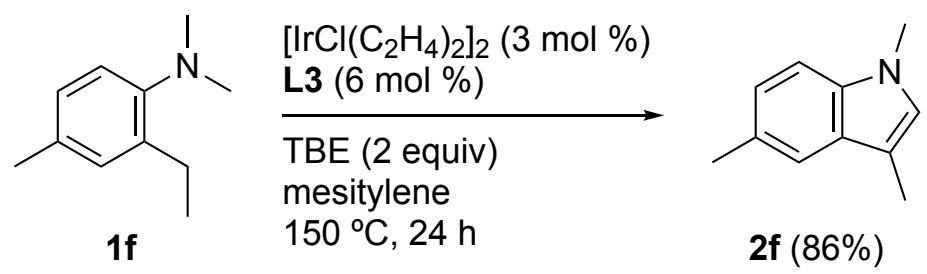

According to the general procedure $A$, the reaction was carried out at $150{ }^{\circ} \mathrm{C}$ for $24 \mathrm{~h}$ using $\left[\operatorname{IrCl}\left(\mathrm{C}_{2} \mathrm{H}_{4}\right)_{2}\right]_{2}(3.11 \mathrm{mg}, 0.00548 \mathrm{mmol}), \mathbf{L 3}(13.4 \mathrm{mg}, 0.0114 \mathrm{mmol}), \mathbf{1 f}(30.5 \mathrm{mg}, 0.187 \mathrm{mmol})$, tert-butylethylene $(31.3 \mathrm{mg}, 0.372 \mathrm{mmol})$, and mesitylene $(0.4 \mathrm{~mL}) .2 \mathrm{f}(25.7 \mathrm{mg}, 0.161 \mathrm{mmol}$, $86 \%$ ) was obtained as a colorless oil after purification by column chromatography on silica gel 
(eluent: hexane:Et $\left.{ }_{2} \mathrm{O}: \mathrm{Et}_{3} \mathrm{~N}=45: 1: 1\right) . \mathbf{2 f}:{ }^{1} \mathrm{H} \mathrm{NMR}\left(400 \mathrm{MHz}, \mathrm{CDCl}_{3}\right) \delta 7.36-7.40(\mathrm{~m}, 1 \mathrm{H}), 7.19$ [d (AB pattern), $J=8.4 \mathrm{~Hz}, 1 \mathrm{H}$ ], 7.07 [dd (AB pattern), $J=8.4,1.6 \mathrm{~Hz}, 1 \mathrm{H}$ ], 6.80 (d, $J=1.2 \mathrm{~Hz}$, 1H), $3.72(\mathrm{~s}, 3 \mathrm{H}), 2.51(\mathrm{~s}, 3 \mathrm{H}), 2.33(\mathrm{~d}, J=1.2 \mathrm{~Hz}, 3 \mathrm{H}) .{ }^{13} \mathrm{C} \mathrm{NMR}\left(101 \mathrm{MHz}, \mathrm{CDCl}_{3}\right) \delta 135.6$, 129.0, 127.8, 126.8, 123.1, 118.7, 109.6, 108.8, 32.6, 21.6, 9.7. HRMS (APCI, positive) $m / z$ calcd for $\mathrm{C}_{11} \mathrm{H}_{14} \mathrm{~N}^{+}[\mathrm{M}+\mathrm{H}]^{+}: 160.1121$, found: 160.1119 .

\section{The Reaction of $1 \mathrm{~g}$ to Afford 5-Acetyl-1,3-dimethyl-1H-indole (2g) (entry 8)}

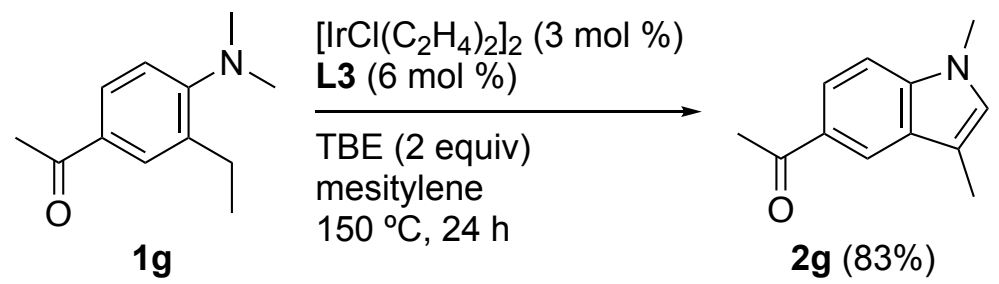

According to the general procedure $A$, the reaction was carried out at $150{ }^{\circ} \mathrm{C}$ for $24 \mathrm{~h}$ using $\left[\operatorname{IrCl}\left(\mathrm{C}_{2} \mathrm{H}_{4}\right)_{2}\right]_{2}(3.39 \mathrm{mg}, 0.00597 \mathrm{mmol}), \mathbf{L 3}(14.2 \mathrm{mg}, 0.0120 \mathrm{mmol}), \mathbf{1 g}(37.6 \mathrm{mg}, 0.197 \mathrm{mmol})$, tert-butylethylene (33.6 mg, $0.399 \mathrm{mmol})$, and mesitylene $(0.4 \mathrm{~mL}) .2 \mathrm{~g}(30.6 \mathrm{mg}, 0.163 \mathrm{mmol}$, $83 \%$ ) was obtained as a white solid after purification by column chromatography on silica gel (eluent: hexane:AcOEt = 3:1). 2g: ${ }^{1} \mathrm{H}$ NMR $\left(400 \mathrm{MHz}, \mathrm{CDCl}_{3}\right) \delta 8.24(\mathrm{~s}, 1 \mathrm{H}), 7.88(\mathrm{~d}, J=8.8 \mathrm{~Hz}$, 1H), $7.26(\mathrm{~d}, J=8.8 \mathrm{~Hz}, 1 \mathrm{H}), 6.87(\mathrm{~s}, 1 \mathrm{H}), 3.74(\mathrm{~s}, 3 \mathrm{H}), 2.68(\mathrm{~s}, 3 \mathrm{H}), 2.36(\mathrm{~s}, 3 \mathrm{H}) .{ }^{13} \mathrm{C}$ NMR $(101$ $\left.\mathrm{MHz}, \mathrm{CDCl}_{3}\right) \delta 198.5,139.5,128.8,128.3,128.1,122.0,121.3,112.5,108.9,32.8,26.7,9.6$. HRMS (APCI, positive) $m / z$ calcd for $\mathrm{C}_{12} \mathrm{H}_{14} \mathrm{NO}^{+}[\mathrm{M}+\mathrm{H}]^{+}: 188.1070$, found: 188.1068 .

\section{The Reaction of $1 \mathrm{~h}$ to Afford 5-Cyano-1,3-dimethyl-1H-indole (2h) (entry 9)}

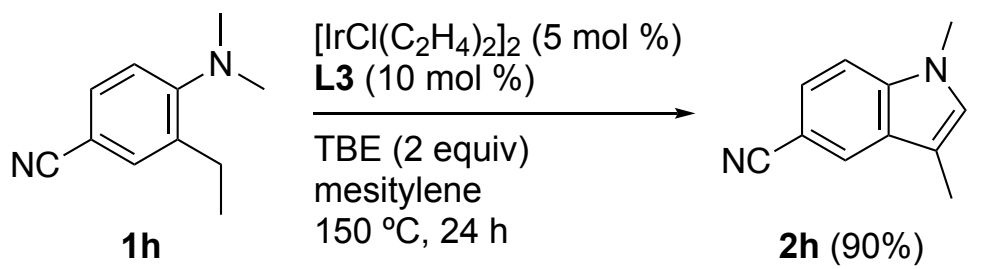

According to the general procedure $A$, the reaction was carried out at $150{ }^{\circ} \mathrm{C}$ for $24 \mathrm{~h}$ using $\left[\operatorname{IrCl}\left(\mathrm{C}_{2} \mathrm{H}_{4}\right)_{2}\right]_{2}(5.85 \mathrm{mg}, 0.0103 \mathrm{mmol}), \mathbf{L 3}(23.7 \mathrm{mg}, 0.0201 \mathrm{mmol}), \mathbf{1 h}(34.9 \mathrm{mg}, 0.200 \mathrm{mmol})$, tert-butylethylene (34.2 mg, $0.406 \mathrm{mmol})$, and mesitylene $(0.4 \mathrm{~mL}) .2 \mathrm{r}(30.4 \mathrm{mg}, 0.179 \mathrm{mmol}$, $90 \%$ ) was obtained as a white solid after purification by column chromatography on silica gel 
(eluent: hexane:AcOEt = 2:1). 2h: ${ }^{1} \mathrm{H}$ NMR (400 MHz, $\left.\mathrm{CDCl}_{3}\right) \delta 7.88(\mathrm{~d}, J=1.6 \mathrm{~Hz}, 1 \mathrm{H}), 7.41$ [dd (AB pattern), $J=8.8,1.6 \mathrm{~Hz}, 1 \mathrm{H}], 7.29$ [d (AB pattern), $J=8.8 \mathrm{~Hz}, 1 \mathrm{H}], 6.93$ (d, $J=1.2 \mathrm{~Hz}$, 1H), $3.76(\mathrm{~s}, 3 \mathrm{H}), 2.31(\mathrm{~d}, J=1.2 \mathrm{~Hz}, 3 \mathrm{H}) .{ }^{13} \mathrm{C} \mathrm{NMR}\left(101 \mathrm{MHz}, \mathrm{CDCl}_{3}\right) \delta 138.5,128.8,128.5$, 124.7, 124.4, 121.2, 111.5, 109.9, 101.5, 32.8, 9.4. HRMS (ESI, positive) $\mathrm{m} / \mathrm{z}$ calcd for $\mathrm{C}_{11} \mathrm{H}_{11} \mathrm{~N}_{2}^{+}[\mathrm{M}+\mathrm{H}]^{+}:$171.0917, found: 171.0918 .

The Reaction of $1 \mathrm{i}$ to Afford 1,3,7-Trimethyl-5-(trimethylsilyl)-1H-indole (2i) (entry 10)

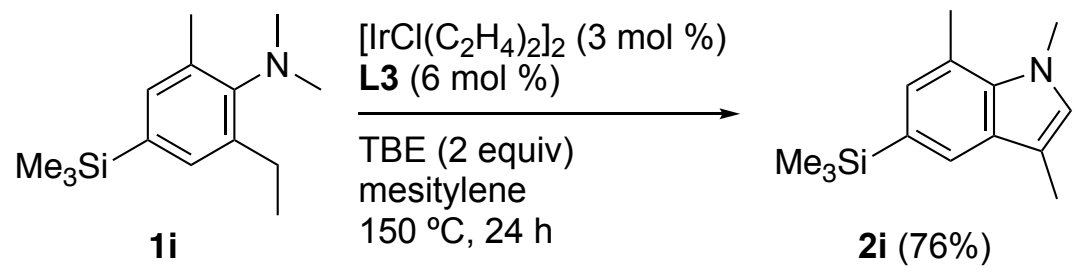

According to the general procedure $A$, the reaction was carried out at $150{ }^{\circ} \mathrm{C}$ for $24 \mathrm{~h}$ using $\left[\operatorname{IrCl}\left(\mathrm{C}_{2} \mathrm{H}_{4}\right)_{2}\right]_{2}(3.40 \mathrm{mg}, 0.00599 \mathrm{mmol}), \mathbf{L 3}(14.2 \mathrm{mg}, 0.0120 \mathrm{mmol}), 1 \mathbf{i}(47.1 \mathrm{mg}, 0.200 \mathrm{mmol})$, tert-butylethylene $(32.8 \mathrm{mg}, 0.390 \mathrm{mmol})$, and mesitylene $(0.4 \mathrm{~mL}) .2 \mathbf{i}(34.9 \mathrm{mg}, 0.151 \mathrm{mmol}$, $76 \%$ including $<2 \%$ of a structural isomer) was obtained as a white solid after purification by column chromatography on silica gel (eluent: hexane:Et $\left.{ }_{2} \mathrm{O}: \mathrm{Et}_{3} \mathrm{~N}=45: 1: 1\right)$. $2 \mathrm{i}:{ }^{1} \mathrm{H}$ NMR (400 $\left.\mathrm{MHz} \mathrm{CDCl}_{3}\right) \delta 7.60(\mathrm{~s}, 1 \mathrm{H}), 7.07(\mathrm{~s}, 1 \mathrm{H}), 6.72(\mathrm{~d}, J=1.2 \mathrm{~Hz}, 1 \mathrm{H}), 4.01(\mathrm{~s}, 3 \mathrm{H}), 2.80(\mathrm{~s}, 3 \mathrm{H})$, $2.35(\mathrm{~d}, J=1.2 \mathrm{~Hz}, 3 \mathrm{H}), 0.36(\mathrm{~s}, 9 \mathrm{H}) .{ }^{13} \mathrm{C} \mathrm{NMR}\left(101 \mathrm{MHz}, \mathrm{CDCl}_{3}\right) \delta 136.5,129.7,129.0,128.8$, 128.3, 122.7, 120.6, 110.0, 36.4, 19.9, 9.6, -0.5. HRMS (APCI, positive) $\mathrm{m} / \mathrm{z}$ calcd for $\mathrm{C}_{14} \mathrm{H}_{22} \mathrm{NSi}^{+}[\mathrm{M}+\mathrm{H}]^{+}: 232.1516$, found: 232.1516 .

\section{The Reaction of $1 \mathrm{j}$ to Afford 5-n-Butyl-1,3,7-trimethyl-1H-indole (2j) (entry 11)}

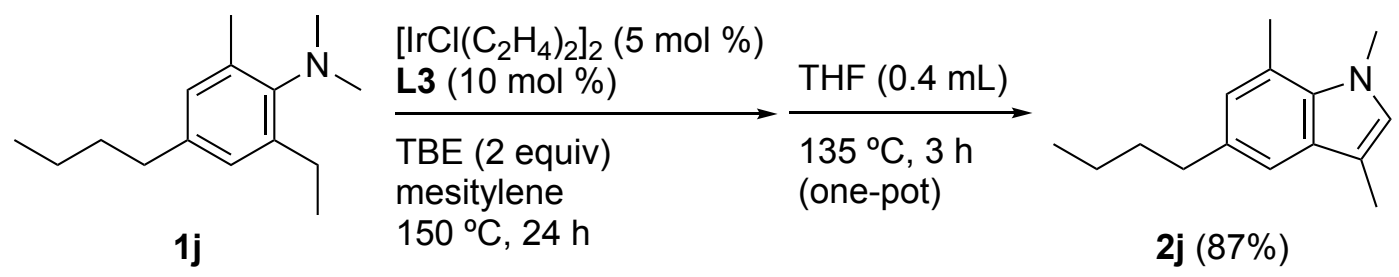

According to the general procedure $B$, the reaction was carried out at $150{ }^{\circ} \mathrm{C}$ for $24 \mathrm{~h}$ using $\left[\operatorname{IrCl}\left(\mathrm{C}_{2} \mathrm{H}_{4}\right)_{2}\right]_{2}(5.46 \mathrm{mg}, 0.00962 \mathrm{mmol}), \mathbf{L 3}(24.0 \mathrm{mg}, 0.0204 \mathrm{mmol}), \mathbf{1 j}$ (39.9 mg, $\left.0.182 \mathrm{mmol}\right)$, tert-butylethylene $(32.6 \mathrm{mg}, 0.387 \mathrm{mmol})$, and mesitylene $(0.4 \mathrm{~mL})$. The resulting reaction mixture was then treated with THF $(0.4 \mathrm{~mL})$ for $3 \mathrm{~h}$ at $135^{\circ} \mathrm{C} . \mathbf{2 j}(34.3 \mathrm{mg}, 0.159 \mathrm{mmol}, 87 \%$ 
including $<5 \%$ of a structural isomer) was obtained as a colorless oil after purification by column chromatography on silica gel (eluent: hexane:Et $\left.{ }_{2} \mathrm{O}: \mathrm{Et}_{3} \mathrm{~N}=40: 1: 1\right) . \quad 2 \mathbf{j}:{ }^{1} \mathrm{H}$ NMR $(400 \mathrm{MHz}$, $\left.\mathrm{CDCl}_{3}\right) \delta 7.20(\mathrm{~s}, 1 \mathrm{H}), 6.78(\mathrm{~s}, 1 \mathrm{H}), 6.70(\mathrm{~d}, J=1.2 \mathrm{~Hz}, 1 \mathrm{H}), 3.98(\mathrm{~s}, 3 \mathrm{H}), 2.75(\mathrm{~s}, 3 \mathrm{H}), 2.70(\mathrm{t}, J$ $=7.6 \mathrm{~Hz}, 2 \mathrm{H}), 2.30(\mathrm{~d}, J=1.2 \mathrm{~Hz}, 3 \mathrm{H}), 1.64-1.73(\mathrm{~m}, 2 \mathrm{H}), 1.36-1.49(\mathrm{~m}, 2 \mathrm{H}), 0.99(\mathrm{t}, J=7.6 \mathrm{~Hz}$, $3 \mathrm{H}) .{ }^{13} \mathrm{C}$ NMR $\left(101 \mathrm{MHz}, \mathrm{CDCl}_{3}\right) \delta 134.4,133.5,130.0,128.4,125.4,120.8,116.1,109.5,36.4$, 35.7, 34.7, 22.7, 19.8, 14.2, 9.6. HRMS (APCI, positive) $\mathrm{m} / z$ calcd for $\mathrm{C}_{15} \mathrm{H}_{22} \mathrm{~N}^{+}[\mathrm{M}+\mathrm{H}]^{+}$: 216.1747, found: 216.1747 .

The Reaction of $1 \mathrm{k}$ to Afford 7-Ethyl-5-methoxy-1,3-dimethyl-1H-indole (2k) (entry 12)

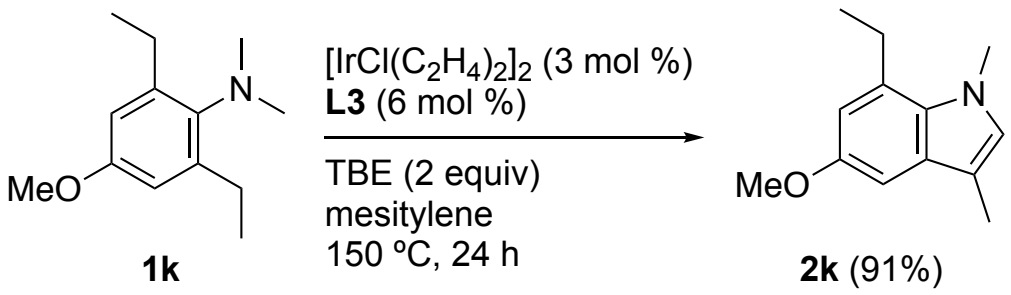

According to the general procedure A, the reaction was carried out at $150{ }^{\circ} \mathrm{C}$ for $24 \mathrm{~h}$ using $\left[\operatorname{IrCl}\left(\mathrm{C}_{2} \mathrm{H}_{4}\right)_{2}\right]_{2}(3.85 \mathrm{mg}, 0.00678 \mathrm{mmol}), \mathbf{L 3}(15.3 \mathrm{mg}, 0.0130 \mathrm{mmol}), \mathbf{1 k}(42.4 \mathrm{mg}, 0.205 \mathrm{mmol})$, tert-butylethylene (37.0 mg, $0.440 \mathrm{mmol})$, and mesitylene $(0.4 \mathrm{~mL}) . \mathbf{2 k}(37.8 \mathrm{mg}, 0.186 \mathrm{mmol}$, 91\%) was obtained as a white solid after purification by column chromatography on silica gel (eluent: hexane:Et $\left.\mathrm{Et}_{2} \mathrm{Et} \mathrm{Et}_{3} \mathrm{~N}=20: 1: 1\right) .2 \mathrm{k}:{ }^{1} \mathrm{H} \mathrm{NMR}\left(400 \mathrm{MHz}, \mathrm{CDCl}_{3}\right) \delta 6.86(\mathrm{~d}, J=2.4 \mathrm{~Hz}, 1 \mathrm{H})$, $6.72(\mathrm{~s}, 1 \mathrm{H}), 6.70(\mathrm{~d}, J=2.4 \mathrm{~Hz}, 1 \mathrm{H}), 3.94(\mathrm{~s}, 3 \mathrm{H}), 3.90$ (s, 3H), 3.08 (q, $J=7.6 \mathrm{~Hz}, 2 \mathrm{H}), 2.30$ (s, $3 \mathrm{H}), 1.37(\mathrm{t}, J=7.6 \mathrm{~Hz}, 3 \mathrm{H}) .{ }^{13} \mathrm{C} \mathrm{NMR}\left(101 \mathrm{MHz}, \mathrm{CDCl}_{3}\right) \delta 153.7,130.5$ (two non-equivalent peaks overlapped), 129.2, 129.1, 112.7, 109.6, 98.2, 55.8, 36.4, 25.5, 16.4, 9.7. ${ }^{13} \mathrm{C}$ NMR (101 $\left.\mathrm{MHz}, \mathrm{C}_{6} \mathrm{D}_{6}\right) \delta 154.6,131.4,130.9,129.0,128.9,113.4,109.6,98.6,55.3,35.7,25.6,16.5,9.9$. HRMS (APCI, positive) $m / z$ calcd for $\mathrm{C}_{13} \mathrm{H}_{18} \mathrm{NO}^{+}[\mathrm{M}+\mathrm{H}]^{+}: 204.1383$, found: 204.1382. 
The Reaction of 11 to Afford 7-Ethyl-5-(dimethylamino)-1,3-dimethyl-1H-indole (2l) (entry

13)

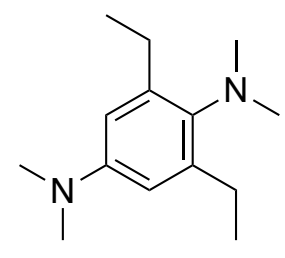

11

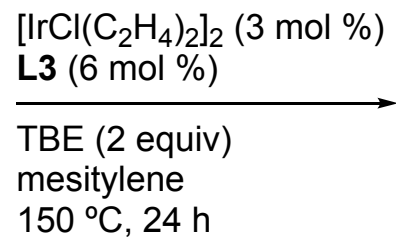

$150^{\circ} \mathrm{C}, 24 \mathrm{~h}$

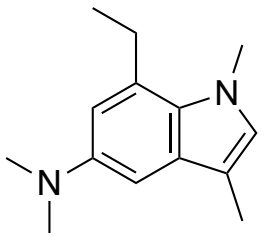

2l (89\%)

According to the general procedure $A$, the reaction was carried out at $150{ }^{\circ} \mathrm{C}$ for $24 \mathrm{~h}$ using $\left[\operatorname{IrCl}\left(\mathrm{C}_{2} \mathrm{H}_{4}\right)_{2}\right]_{2}(3.33 \mathrm{mg}, 0.00587 \mathrm{mmol}), \mathbf{L 3}(14.0 \mathrm{mg}, 0.0119 \mathrm{mmol}), 1 \mathrm{l}(44.1 \mathrm{mg}, 0.200 \mathrm{mmol})$, tert-butylethylene (34.4 mg, $0.409 \mathrm{mmol})$, and mesitylene (0.4 mL). 21 (38.2 mg, $0.177 \mathrm{mmol}$, $89 \%$ ) was obtained as a yellow oil after purification by column chromatography on silica gel (eluent: hexane: $\left.\mathrm{Et}_{2} \mathrm{O}: \mathrm{Et}_{3} \mathrm{~N}=20: 1: 1\right) .2 \mathrm{l}:{ }^{1} \mathrm{H} \mathrm{NMR}\left(400 \mathrm{MHz}, \mathrm{CDCl}_{3}\right) \delta 6.80(\mathrm{~d}, J=2.4 \mathrm{~Hz}, 1 \mathrm{H})$, $6.74(\mathrm{~d}, J=2.4 \mathrm{~Hz}, 1 \mathrm{H}), 6.69(\mathrm{~d}, J=0.8 \mathrm{~Hz}, 1 \mathrm{H}), 3.93(\mathrm{~s}, 3 \mathrm{H}), 3.08$ (q, $J=7.6 \mathrm{~Hz}, 2 \mathrm{H}), 2.96$ (s, $6 \mathrm{H}), 2.29(\mathrm{~d}, J=0.8 \mathrm{~Hz}, 3 \mathrm{H}), 1.38(\mathrm{t}, J=7.6 \mathrm{~Hz}, 3 \mathrm{H}) .{ }^{13} \mathrm{C} \mathrm{NMR}\left(101 \mathrm{MHz}, \mathrm{CDCl}_{3}\right) \delta 145.6$, 130.7, 129.8, 128.9, 128.4, 114.1, 109.3, 101.5, 43.2, 36.3, 25.9, 16.8, 9.7. HRMS (APCI, positive) $m / z$ calcd for $\mathrm{C}_{14} \mathrm{H}_{21} \mathrm{~N}_{2}^{+}[\mathrm{M}+\mathrm{H}]^{+}: 217.1699$, found: 217.1698.

\section{The Reaction of $1 \mathrm{~m}$ to Afford 6-Cloro-7-ethyl-1,3-dimethyl-1H-indole (2m) (entry 14)}
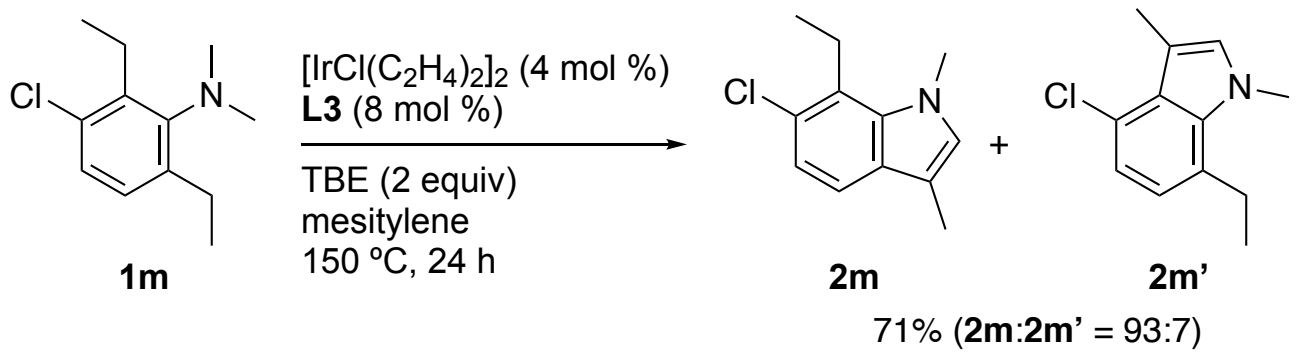

According to the general procedure $A$, the reaction was carried out at $150{ }^{\circ} \mathrm{C}$ for $24 \mathrm{~h}$ using $\left[\operatorname{IrCl}\left(\mathrm{C}_{2} \mathrm{H}_{4}\right)_{2}\right]_{2}(4.31 \mathrm{mg}, 0.00759 \mathrm{mmol}), \mathbf{L 3}(18.8 \mathrm{mg}, 0.0159 \mathrm{mmol}), \mathbf{1 m}(41.3 \mathrm{mg}, 0.195 \mathrm{mmol})$, tert-butylethylene (31.9 mg, $0.379 \mathrm{mmol})$, and mesitylene $(0.4 \mathrm{~mL}) .2 \mathrm{~m}(28.9 \mathrm{mg}, 0.139 \mathrm{mmol}$, $71 \%$, containing $7 \%$ of an isomer $\mathbf{2 m}$ ') was obtained as a yellow oil after purification by column chromatography on silica gel (eluent: hexane: $\left.\mathrm{CH}_{2} \mathrm{Cl}_{2}: \mathrm{Et}_{3} \mathrm{~N}=45: 3: 2\right) .2 \mathbf{m}:{ }^{1} \mathrm{H} \mathrm{NMR}(400 \mathrm{MHz}$, $\left.\mathrm{CDCl}_{3}\right) \delta 7.30[\mathrm{~d}(\mathrm{AB}$ pattern), $J=8.4 \mathrm{~Hz}, 1 \mathrm{H}$ ], 7.11 [d (AB pattern), $J=8.4 \mathrm{~Hz}, 1 \mathrm{H}$ ], $6.72(\mathrm{~d}, J$ $=1.2 \mathrm{~Hz}, 1 \mathrm{H}), 3.97$ (s, 3H), $3.23(\mathrm{q}, J=7.2 \mathrm{~Hz}, 2 \mathrm{H}), 2.27(\mathrm{~d}, J=1.2 \mathrm{~Hz}, 3 \mathrm{H}), 1.32(\mathrm{t}, J=7.2 \mathrm{~Hz}$, 
3H). ${ }^{13} \mathrm{C}$ NMR (101 MHz, $\left.\mathrm{CDCl}_{3}\right) \delta 135.4,129.5,129.2,128.2,125.2,120.8,117.5,110.1,36.7$, 21.8, 15.6, 9.4. HRMS (APCI, positive) $m / z$ calcd for $\mathrm{C}_{12} \mathrm{H}_{15} \mathrm{ClN}^{+}[\mathrm{M}+\mathrm{H}]^{+}: 208.0888$, found: 208.0887. A mixture of $\mathbf{2 m}$ and $\mathbf{2 m}$ ' was used for HRMS measurement. 2m': ${ }^{1} \mathrm{H}$ NMR (400 $\left.\mathrm{MHz}, \mathrm{CDCl}_{3}\right) \delta 6.95$ [d (AB pattern), $J=8.0 \mathrm{~Hz}, 1 \mathrm{H}$ ], 6.83 [d (AB pattern), $J=8.0 \mathrm{~Hz}, 1 \mathrm{H}$ ], 6.71 (d, $J=1.2 \mathrm{~Hz}, 1 \mathrm{H}), 3.94(\mathrm{~s}, 3 \mathrm{H}), 3.05$ (q, $J=7.6 \mathrm{~Hz}, 2 \mathrm{H}), 2.53$ (d, $J=1.2 \mathrm{~Hz}, 3 \mathrm{H}), 1.32$ (t, $J=$ $7.6 \mathrm{~Hz}, 3 \mathrm{H})$.

\section{The Reaction of $1 \mathrm{~m}$ to Afford 7-Ethyl-6-(phthalimidomethyl)-1,3-dimethyl-1H-indole (2n) (entry 15)}
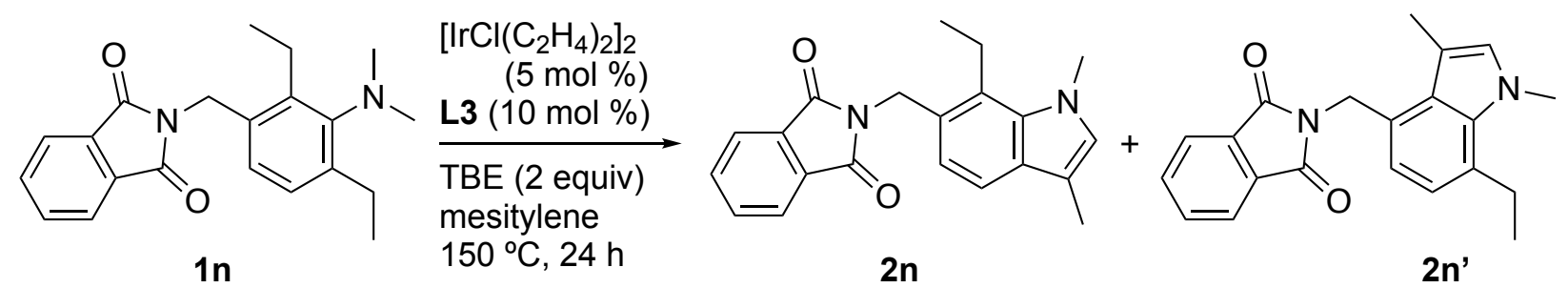

$89 \%(2 n: 2 n '=91: 9)$

According to the general procedure A, the reaction was carried out at $150{ }^{\circ} \mathrm{C}$ for $24 \mathrm{~h}$ using $\left[\operatorname{IrCl}\left(\mathrm{C}_{2} \mathrm{H}_{4}\right)_{2}\right]_{2}(5.63 \mathrm{mg}, 0.00992 \mathrm{mmol}), \mathbf{L 3}(23.7 \mathrm{mg}, 0.0201 \mathrm{mmol}), \mathbf{1 n}(67.0 \mathrm{mg}, 0.199 \mathrm{mmol})$, tert-butylethylene (34.2 mg, $0.406 \mathrm{mmol})$, and mesitylene $(0.4 \mathrm{~mL}) .2 \mathrm{n}(59.0 \mathrm{mg}, 0.177 \mathrm{mmol}$, $89 \%$, containing $9 \%$ of an isomer $\mathbf{2 n}$ ') was obtained as a yellow solid after purification by column chromatography on silica gel (eluent: hexane:AcOEt = 3:1). 2n: ${ }^{1} \mathrm{H}$ NMR (400 MHz, $\left.\mathrm{CDCl}_{3}\right) \delta 7.81-7.87(\mathrm{~m}, 2 \mathrm{H}), 7.66-7.72(\mathrm{~m}, 2 \mathrm{H}), 7.34$ [d (AB pattern), $\left.J=8.4 \mathrm{~Hz}, 1 \mathrm{H}\right], 7.17$ [d (AB pattern), $J=8.4 \mathrm{~Hz}, 1 \mathrm{H}], 6.71(\mathrm{~d}, J=1.2 \mathrm{~Hz}, 1 \mathrm{H}), 5.05$ (s, 2H), 3.99 (s, 3H), 3.40 (q, $J=7.6$ $\mathrm{Hz}, 2 \mathrm{H}), 2.25(\mathrm{~d}, J=1.2 \mathrm{~Hz}, 3 \mathrm{H}), 1.36(\mathrm{t}, J=7.6 \mathrm{~Hz}, 3 \mathrm{H}) .{ }^{13} \mathrm{C} \mathrm{NMR}\left(101 \mathrm{MHz}, \mathrm{CDCl}_{3}\right) \delta 168.5$, 135.0, 134.0, 132.3, 130.1, 129.8, 127.4, 126.5, 123.3, 120.9, 116.9, 109.7, 39.0, 36.8, 20.2, 17.1, 9.4. HRMS (APCI, positive) $\mathrm{m} / z$ calcd for $\mathrm{C}_{21} \mathrm{H}_{21} \mathrm{~N}_{2} \mathrm{O}_{2}{ }^{+}[\mathrm{M}+\mathrm{H}]^{+}: 333.1598$, found: 333.1601. A mixture of $\mathbf{2 n}$ and $2 \mathbf{n}$ ' was used for HRMS measurement. 2n': ${ }^{1} \mathrm{H}$ NMR (400 $\mathrm{MHz}, \mathrm{CDCl}_{3}$ ) $\delta 7.87-7.91(\mathrm{~m}, 2 \mathrm{H}), 7.71-7.75(\mathrm{~m}, 2 \mathrm{H}), 6.82$ [d (AB pattern), $J=7.6 \mathrm{~Hz}, 1 \mathrm{H}], 6.73(\mathrm{~d}, J=1.2 \mathrm{~Hz}$, 1H), 6.68 [d (AB pattern), $J=7.6 \mathrm{~Hz}, 1 \mathrm{H}], 5.43$ (s, 2H), 3.94 (s, 3H), 3.04 (q, $J=7.6 \mathrm{~Hz}, 2 \mathrm{H})$, $2.65(\mathrm{~d}, J=1.2 \mathrm{~Hz}, 3 \mathrm{H}), 1.30(\mathrm{t}, J=7.6 \mathrm{~Hz}, 3 \mathrm{H}) .{ }^{13} \mathrm{C} \mathrm{NMR}\left(101 \mathrm{MHz}, \mathrm{CDCl}_{3}\right) \delta 168.5,135.8$, 134.0, 132.3, 130.2, 127.2, 127.1, 127.0, 123.4, 122.5, 116.6, 109.9, 38.9, 36.6, 25.4, 16.7, 13.7. 
The Reaction of 1n to Afford Bis(1,3-dimethyl-1H-indol-5-yl)methane (2o) (entry 16)

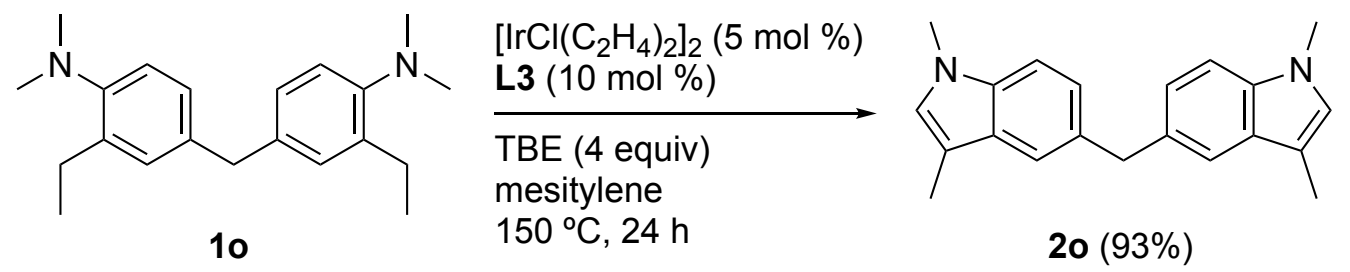

According to the general procedure $A$, the reaction was carried out at $150{ }^{\circ} \mathrm{C}$ for $24 \mathrm{~h}$ using $\left[\mathrm{IrCl}\left(\mathrm{C}_{2} \mathrm{H}_{4}\right)_{2}\right]_{2}(5.37 \mathrm{mg}, 0.00946 \mathrm{mmol}), \mathbf{L 3}(22.6 \mathrm{mg}, 0.0192 \mathrm{mmol}), 10$ (58.1 mg, $\left.0.187 \mathrm{mmol}\right)$, tert-butylethylene $(67.7 \mathrm{mg}, 0.804 \mathrm{mmol})$, and mesitylene $(0.4 \mathrm{~mL}) .20(52.3 \mathrm{mg}, 0.173 \mathrm{mmol}$, 93\%) was obtained as a white solid after purification by column chromatography on silica gel (eluent: hexane: $\left.\mathrm{CH}_{2} \mathrm{Cl}_{2}: \mathrm{Et}_{3} \mathrm{~N}=10: 1: 1\right) .20:{ }^{1} \mathrm{H} \mathrm{NMR}\left(400 \mathrm{MHz}, \mathrm{CDCl}_{3}\right) \delta 7.52(\mathrm{~d}, J=1.2 \mathrm{~Hz}$, 2H), 7.24 [d (AB pattern), $J=8.4 \mathrm{~Hz}, 2 \mathrm{H}$ ], 7.17 [dd (AB pattern), $J=8.4,1.2 \mathrm{~Hz}, 2 \mathrm{H}$ ], 6.83 (d, $J$ $=1.2 \mathrm{~Hz}, 2 \mathrm{H}), 4.31(\mathrm{~s}, 2 \mathrm{H}), 3.74(\mathrm{~s}, 6 \mathrm{H}), 2.37(\mathrm{~d}, J=1.2 \mathrm{~Hz}, 6 \mathrm{H}) .{ }^{13} \mathrm{C} \mathrm{NMR}\left(101 \mathrm{MHz}, \mathrm{CDCl}_{3}\right) \delta$ 135.8, 132.7, 128.8, 126.7, 123.2, 118.9, 109.9, 109.0, 42.4, 32.6, 9.7. HRMS (APCI, positive) $m / z$ calcd for $\mathrm{C}_{21} \mathrm{H}_{23} \mathrm{~N}_{2}^{+}[\mathrm{M}+\mathrm{H}]^{+}: 303.1856$, found: 303.1860 .

\section{The Reaction of 10 to Afford Bis(1,3,7-trimethyl-1H-indol-5-yl)methane (2p) (entry 17)}
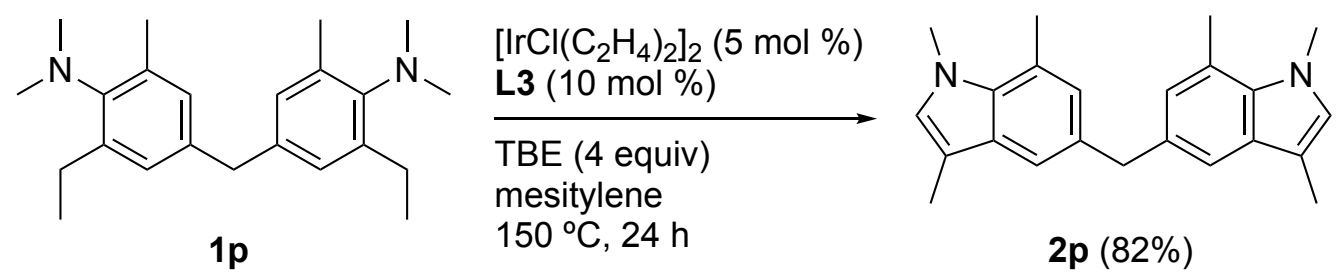

According to the general procedure $A$, the reaction was carried out at $150{ }^{\circ} \mathrm{C}$ for $24 \mathrm{~h}$ using $\left[\mathrm{IrCl}\left(\mathrm{C}_{2} \mathrm{H}_{4}\right)_{2}\right]_{2}(5.61 \mathrm{mg}, 0.00988 \mathrm{mmol}), \mathbf{L 3}(23.9 \mathrm{mg}, 0.0203 \mathrm{mmol}), \mathbf{1 p}(66.5 \mathrm{mg}, 0.196 \mathrm{mmol})$, tert-butylethylene $(67.9 \mathrm{mg}, 0.807 \mathrm{mmol})$, and mesitylene $(0.4 \mathrm{~mL}) .2 p(53.2 \mathrm{mg}, 0.161 \mathrm{mmol}$, $82 \%$ including $<10 \%$ of a structural isomer) was obtained as a white crystal after purification by column chromatography on silica gel (eluent: hexane: $\left.\mathrm{CH}_{2} \mathrm{Cl}_{2}: \mathrm{Et}_{3} \mathrm{~N}=10: 1: 1\right)$. 2p: ${ }^{1} \mathrm{H} \mathrm{NMR}(400$ $\left.\mathrm{MHz} \mathrm{CDCl}_{3}\right) \delta 7.25(\mathrm{~s}, 2 \mathrm{H}), 6.78(\mathrm{~s}, 2 \mathrm{H}), 6.67(\mathrm{~d}, J=1.2 \mathrm{~Hz}, 2 \mathrm{H}), 4.09(\mathrm{~s}, 2 \mathrm{H}), 3.96(\mathrm{~s}, 6 \mathrm{H})$, $2.68(\mathrm{~s}, 6 \mathrm{H}), 2.26(\mathrm{~d}, J=1.2 \mathrm{~Hz}, 6 \mathrm{H}) .{ }^{13} \mathrm{C} \mathrm{NMR}\left(101 \mathrm{MHz}, \mathrm{CDCl}_{3}\right) \delta 134.5,133.0,129.9,128.3$, 125.9, 121.0, 116.9, 109.7, 42.0, 36.4, 19.8, 9.7. HRMS (APCI, positive) $m / z$ calcd for $\mathrm{C}_{23} \mathrm{H}_{27} \mathrm{~N}_{2}{ }^{+}$ $[\mathrm{M}+\mathrm{H}]^{+}:$331.2169, found: 331.2173 . 
The Reaction of 1p to Afford Bis(1,3,7-trimethyl-1H-indol-5-yl)methane (2q) (entry 18)

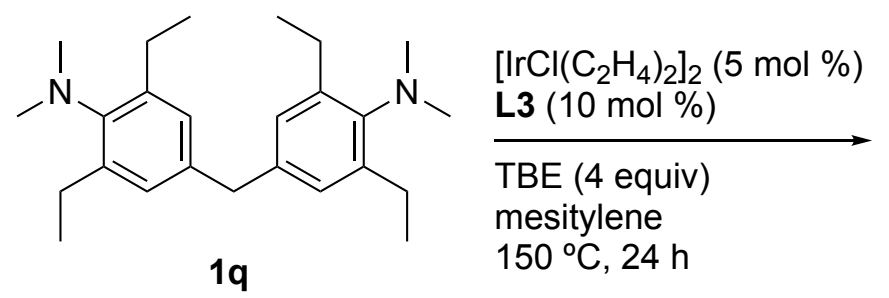

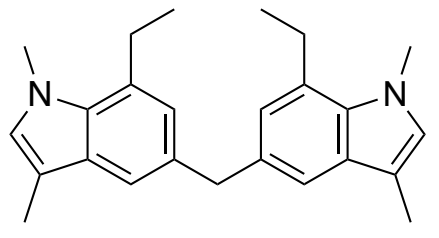

$2 q(93 \%)$

According to the general procedure $A$, the reaction was carried out at $150{ }^{\circ} \mathrm{C}$ for $24 \mathrm{~h}$ using $\left[\operatorname{IrCl}\left(\mathrm{C}_{2} \mathrm{H}_{4}\right)_{2}\right]_{2}(5.68 \mathrm{mg}, 0.0100 \mathrm{mmol}), \mathbf{L 3}(24.0 \mathrm{mg}, 0.0204 \mathrm{mmol}), \mathbf{1 q}(72.6 \mathrm{mg}, 0.198 \mathrm{mmol})$, tert-butylethylene $(67.8 \mathrm{mg}, 0.806 \mathrm{mmol})$, and mesitylene $(0.4 \mathrm{~mL}) .2 q(66.0 \mathrm{mg}, 0.184 \mathrm{mmol}$, 93\%) was obtained as a white solid after purification by column chromatography on silica gel (eluent: hexane: $\left.\mathrm{CH}_{2} \mathrm{Cl}_{2}: \mathrm{Et}_{3} \mathrm{~N}=10: 1: 1\right) .2 q:{ }^{1} \mathrm{H} \mathrm{NMR}\left(400 \mathrm{MHz}, \mathrm{CDCl}_{3}\right) \delta 7.27(\mathrm{~d}, J=1.6 \mathrm{~Hz}$, 2H), $6.89(\mathrm{~d}, J=1.6 \mathrm{~Hz}, 2 \mathrm{H}), 6.70(\mathrm{~d}, J=0.8 \mathrm{~Hz}, 2 \mathrm{H}), 4.17(\mathrm{~s}, 2 \mathrm{H}), 3.94(\mathrm{~s}, 6 \mathrm{H}), 3.04$ (q, $J=7.6$ $\mathrm{Hz}, 4 \mathrm{H}), 2.27(\mathrm{~d}, J=0.8 \mathrm{~Hz}, 6 \mathrm{H}), 1.32(\mathrm{t}, J=7.6 \mathrm{~Hz}, 6 \mathrm{H}) .{ }^{13} \mathrm{C} \mathrm{NMR}\left(101 \mathrm{MHz}, \mathrm{CDCl}_{3}\right) \delta 133.6$, $132.9,130.4,128.5,127.6,124.4,116.9,109.9,42.0,36.3,25.6,17.1,9.7$. HRMS (APCI, positive) $m / z$ calcd for $\mathrm{C}_{25} \mathrm{H}_{31} \mathrm{~N}_{2}^{+}[\mathrm{M}+\mathrm{H}]^{+}: 359.2482$, found: 359.2486 .

\section{Preliminary Investigations for Functional Group Compatibility}

To check functional group compatibility, we carried out the reaction of $\mathbf{1 a}$ in the presence of functionalized benzenes (1 equiv) (Table S2). No reaction took place with bromobenzene (entry 2) probably due to the loss of catalytic activity by oxidative addition of the $\mathrm{C}-\mathrm{Br}$ bond into $\operatorname{Ir}(\mathrm{I})$. Similar deactivation of the catalyst was also observed in the reaction with 2-bromotoluene and chlorobenzene (entries 3 and 4), whereas 2-chlorotoluene did not interfere the reaction (entry 5). These results indicate that sterically shielded halogen is less likely to affect the catalyst. The reaction with nitrobenzene did not proceed probably due to strong coordination of the nitro group to the iridium center prevent the coordination of 1a (entry 6). 
Table S2. The Reaction of 1a in the presence of a Functionalized Benzenes ${ }^{a}$

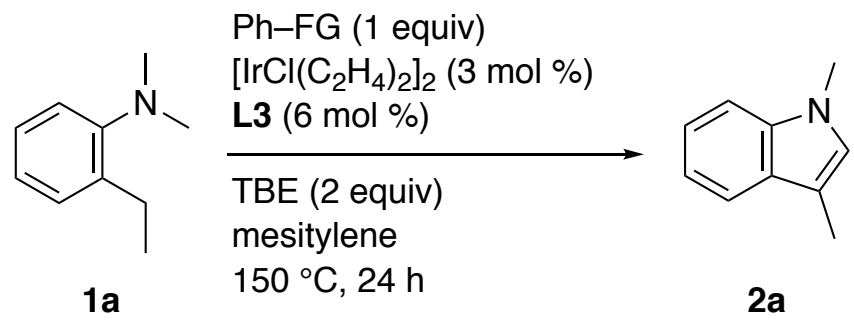

\begin{tabular}{clc}
\hline entry & Ph-FG & \% yield of $2 \mathbf{a}^{b}$ \\
\hline 1 & none & $93(84)^{c}$ \\
2 & Bromobenzene & no reaction \\
3 & 2-Bromotoluene & 49 \\
4 & Chlorobenzene & 16 \\
5 & 2-Chlorotoluene & 92 \\
6 & Nitrobenzene & no reaction \\
\hline
\end{tabular}

a 1 a $(0.10 \mathrm{mmol}),\left[\mathrm{IrCl}\left(\mathrm{C}_{2} \mathrm{H}_{4}\right)_{2}\right]_{2}(3 \mathrm{~mol} \%)$, L3 $(6 \mathrm{~mol} \%)$, TBE (2 equiv), and mesitylene $(0.2 \mathrm{~mL})$ were stirred at $150{ }^{\circ} \mathrm{C}$ for $24 \mathrm{~h} .{ }^{b}$ Determined by ${ }^{1} \mathrm{H}$ NMR. ${ }^{c}$ Isolated yield in the 0.2 mmol scale reaction.

\section{The Reaction of $1 r$}
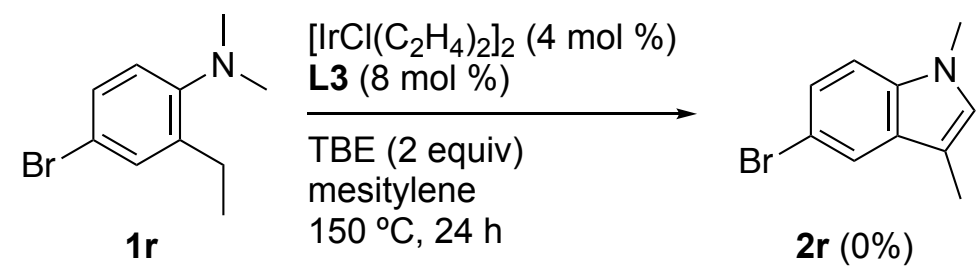

According to the general procedure $A$, the reaction was carried out at $150{ }^{\circ} \mathrm{C}$ for $24 \mathrm{~h}$ using $\left[\operatorname{IrCl}\left(\mathrm{C}_{2} \mathrm{H}_{4}\right)_{2}\right]_{2}(2.41 \mathrm{mg}, 0.00425 \mathrm{mmol}), \mathbf{L 3}(8.92 \mathrm{mg}, 0.0756 \mathrm{mmol}), \mathbf{1 r}(26.9 \mathrm{mg}, 0.118 \mathrm{mmol})$, tert-butylethylene $(18.8 \mathrm{mg}, 0.223 \mathrm{mmol})$, and mesitylene $(0.2 \mathrm{~mL})$. No reaction took place.

\section{Reactions of Other Anilines and an Indoline (Figure 1)}

\section{The Reaction of 8 (entry 1)}

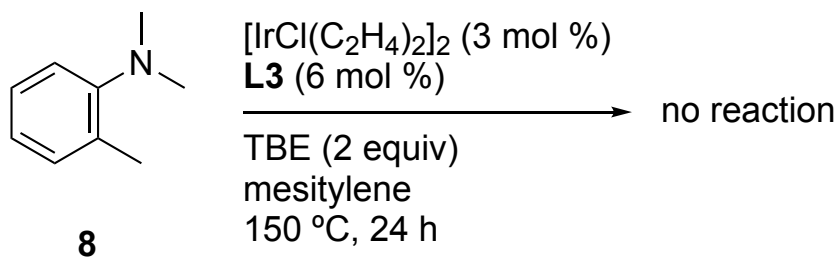

According to the general procedure $A$ described in section 5 , the reaction was carried out at $150{ }^{\circ} \mathrm{C}$ for $24 \mathrm{~h}$ using $\left[\mathrm{IrCl}\left(\mathrm{C}_{2} \mathrm{H}_{4}\right)_{2}\right]_{2}(1.60 \mathrm{mg}, 0.00282 \mathrm{mmol}), \mathbf{L 3}(7.38 \mathrm{mg}, 0.00626 \mathrm{mmol}), 8$ 
(14.7 mg, $0.109 \mathrm{mmol})$, tert-butylethylene $(18.2 \mathrm{mg}, 0.216 \mathrm{mmol})$, and mesitylene $(0.2 \mathrm{~mL})$. No reaction took place.

\section{The Reaction of 3a to Afford 2a (entry 2)}

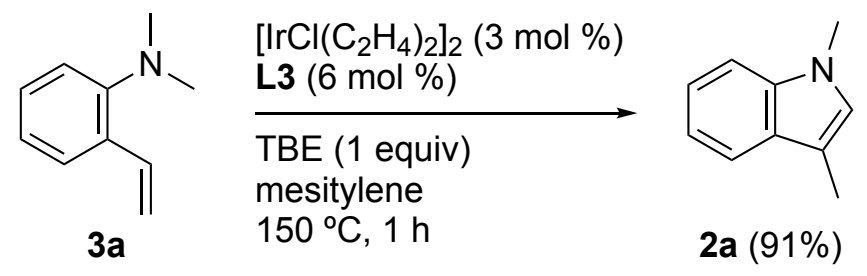

According to the general procedure $A$ described in section 5 , the reaction was carried out at $150{ }^{\circ} \mathrm{C}$ for $1 \mathrm{~h}$ using $\left[\operatorname{IrCl}\left(\mathrm{C}_{2} \mathrm{H}_{4}\right)_{2}\right]_{2}(4.20 \mathrm{mg}, 0.00740 \mathrm{mmol}), \mathbf{L 3}(17.1 \mathrm{mg}, 0.0145 \mathrm{mmol}), 3 \mathbf{a}$ (37.7 mg, $0.256 \mathrm{mmol})$, tert-butylethylene $(21.2 \mathrm{mg}, 0.252 \mathrm{mmol})$, and mesitylene $(0.4 \mathrm{~mL}) .2 \mathrm{a}$ (33.8 mg, 91\%) was obtained as a colorless oil after purification by column chromatography on silica gel (eluent: hexane: $\mathrm{Et}_{2} \mathrm{O}: \mathrm{Et}_{3} \mathrm{~N}=45: 1: 1$ ).

\section{The Reaction of 9 to Afford 2-Ethenyl- $N, N$-dibenzylaniline (10) (entry 3)}<smiles>CCc1ccccc1N(Cc1ccccc1)Cc1ccccc1</smiles>

9
$\left[\mathrm{IrCl}\left(\mathrm{C}_{2} \mathrm{H}_{4}\right)_{2}\right]_{2}(3 \mathrm{~mol} \%)$

L3 $(6 \mathrm{~mol} \%)$

TBE (2 equiv) mesitylene $150^{\circ} \mathrm{C}, 51 \mathrm{~h}$

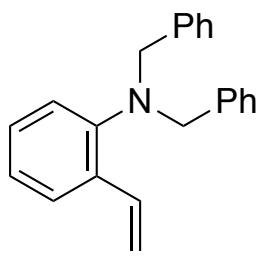

$10(44 \%, N M R)$

According to the general procedure $A$ described in section 5, the reaction was carried out at $150{ }^{\circ} \mathrm{C}$ for $51 \mathrm{~h}$ using $\left[\mathrm{IrCl}\left(\mathrm{C}_{2} \mathrm{H}_{4}\right)_{2}\right]_{2}(1.70 \mathrm{mg}, 0.00300 \mathrm{mmol}), \mathbf{L 3}(7.02 \mathrm{mg}, 0.00600 \mathrm{mmol}), 9$ (26.5 mg, $0.0879 \mathrm{mmol})$, tert-butylethylene (19.9 mg, $0.236 \mathrm{mmol})$, and mesitylene $(0.2 \mathrm{~mL}) .{ }^{1} \mathrm{H}$ NMR analysis of the resulting solution (internal standard: diphenylmethane) indicated that no cyclization took place, although formation of the transfer dehydrogenation product 10 (44\%) was observed. 10 was isolated as a colorless oil after purification by HPLC [column: LiChlosorb CN ( $\phi 25 \mathrm{~mm}$ x $250 \mathrm{~mm}, 7 \mu \mathrm{m}$ ); eluent: hexane only] and Kugelrohr distillation. 10: ${ }^{1} \mathrm{H}$ NMR (400 $\left.\mathrm{MHz} \mathrm{CDCl}_{3}\right) \delta 7.54(\mathrm{dd}, J=8.0,1.6 \mathrm{~Hz}, 1 \mathrm{H}), 7.39(\mathrm{dd}, J=17.6,10.8 \mathrm{~Hz}, 1 \mathrm{H}), 7.20-7.32(\mathrm{~m}$, $10 \mathrm{H}), 7.12(\mathrm{td}, J=7.6,1.6 \mathrm{~Hz}, 1 \mathrm{H}), 7.00-7.06(\mathrm{~m}, 1 \mathrm{H}), 6.86(\mathrm{dd}, J=8.0,0.8 \mathrm{~Hz}, 1 \mathrm{H}), 5.74(\mathrm{dd}, J$ $=17.6,1.6 \mathrm{~Hz}, 1 \mathrm{H}), 5.32(\mathrm{dd}, J=10.8,1.6 \mathrm{~Hz}, 1 \mathrm{H}), 4.13(\mathrm{~s}, 4 \mathrm{H}) \cdot{ }^{13} \mathrm{C} \mathrm{NMR}\left(101 \mathrm{MHz}, \mathrm{CDCl}_{3}\right) \delta$ 
$149.1,138.2,134.7,133.2,128.8,128.3,128.1,127.1,126.9,123.2,122.1,113.9,56.7$. HRMS (APCI, positive) $m / z$ calcd for $\mathrm{C}_{22} \mathrm{H}_{22} \mathrm{~N}^{+}[\mathrm{M}+\mathrm{H}]^{+}: 300.1747$, found: 300.1751 .

\section{The Reaction of 11a to Afford 2a (entry 4)}<smiles>CC1CN(C)c2ccccc21</smiles>

$11 \mathrm{a}$

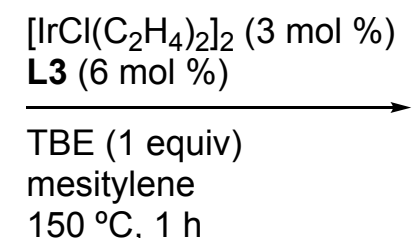

$150^{\circ} \mathrm{C}, 1 \mathrm{~h}$

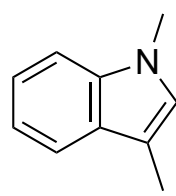

$2 a(92 \%)$

According to the general procedure $A$ described in section 5 , the reaction was carried out at $150{ }^{\circ} \mathrm{C}$ for $1 \mathrm{~h}$ using $\left[\operatorname{IrCl}\left(\mathrm{C}_{2} \mathrm{H}_{4}\right)_{2}\right]_{2}(3.74 \mathrm{mg}, 0.00659 \mathrm{mmol}), \mathbf{L 3}(14.6 \mathrm{mg}, 0.0124 \mathrm{mmol}), 11 \mathrm{a}$ (29.1 mg, $0.198 \mathrm{mmol})$, tert-butylethylene (17.5 mg, $0.208 \mathrm{mmol})$, and mesitylene (0.4 mL). 2a (26.5 mg, $0.182 \mathrm{mmol}, 92 \%$ ) was obtained as a colorless oil after purification by column chromatography on silica gel (eluent: hexane: $\mathrm{Et}_{2} \mathrm{O}: \mathrm{Et}_{3} \mathrm{~N}=45: 1: 1$ ).

\section{An Additional Result involving Reaction Mechanism (ref. 20 in the text)}

The reaction of $\mathbf{1 a}$ was examined using an isolated $[(\mathbf{L 3}) \mathrm{IrCl}]_{2}$ complex $^{8 \mathrm{c}}$ as a catalyst. The reaction afforded $\mathbf{2 a}$ in $83 \%$ yield, which is almost comparable to the result obtained by $\left[\operatorname{IrCl}\left(\mathrm{C}_{2} \mathrm{H}_{4}\right)_{2}\right]_{2}$ with $\mathbf{L 3}$.<smiles>CCc1ccccc1N(C)C</smiles>

$1 \mathrm{a}$
$[(\mathrm{L} 3) \mathrm{IrCl}]_{2}(3 \mathrm{~mol} \%)$

TBE (2 equiv) mesitylene $150^{\circ} \mathrm{C}, 24 \mathrm{~h}$

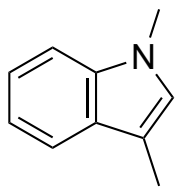

$2 a$

$83 \%$ yield (NMR)

cf. $\left[\mathrm{IrCl}\left(\mathrm{C}_{2} \mathrm{H}_{4}\right)_{2}\right]_{2}(3 \mathrm{~mol} \%) / \mathrm{L3}(6 \mathrm{~mol} \%)$ afforded $2 \mathrm{a}$ in 93\% yield (NMR). 


\section{Reaction of D-Labeled Substrates (Figure 2)}

\section{7-1. Preparation of D-Labeled Substrates}

2-(Ethyl-1,1-d $)-N, N$-dimethylaniline (1a-d $)$

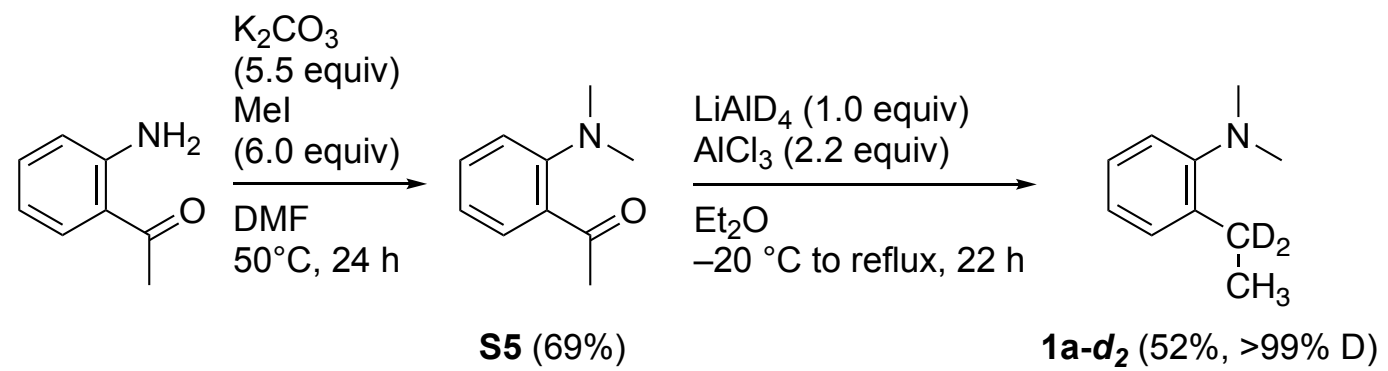

An oven dried $500 \mathrm{~mL}$ three-neck flask, equipped with a magnetic stirring bar, a rubber septum, and a three-way stopcock, was charged with potassium carbonate $(11.5 \mathrm{~g}, 83.2 \mathrm{mmol})$. The flask was evacuated and backfilled with nitrogen. DMF (30 mL), 2-acetylaniline (TCI, 2.05 g, $15.2 \mathrm{mmol})$, and methyl iodide $(13.7 \mathrm{~g}, 96.5 \mathrm{mmol})$ were added to the flask. The mixture was stirred for $24 \mathrm{~h}$ at $50{ }^{\circ} \mathrm{C}$. Hexane $(40 \mathrm{~mL})$, AcOEt $(40 \mathrm{~mL})$, and water $(50 \mathrm{~mL})$ were added to the flask. The organic materials were collected, washed with water $(50 \mathrm{~mL} \mathrm{x} \mathrm{4)}$ and brine $(50 \mathrm{~mL} \mathrm{x}$ 2), and dried over anhydrous magnesium sulfate. The crude product was purified by column chromatography on silica gel (SiliaFlash; eluent: hexane: $\left.\mathrm{Et}_{2} \mathrm{O}=90: 10\right)$ and Kugelrhor distillation. S5 (1.71 g, 10.5 mmol, 69\% yield) was obtained as a yellow liquid. S5: ${ }^{1} \mathrm{H}$ NMR (400 MHz, $\left.\mathrm{CDCl}_{3}\right) \delta 7.41(\mathrm{dd}, J=7.6,1.6 \mathrm{~Hz}, 1 \mathrm{H}), 7.34(\mathrm{ddd}, J=8.4,7.6,1.6 \mathrm{~Hz}, 1 \mathrm{H}), 6.98(\mathrm{~d}, J=8.4 \mathrm{~Hz}$, $1 \mathrm{H}), 6.91(\mathrm{td}, J=7.6,0.8 \mathrm{~Hz}, 1 \mathrm{H}), 2.78(\mathrm{~s}, 6 \mathrm{H}), 2.58(\mathrm{~s}, 3 \mathrm{H}) \cdot{ }^{13} \mathrm{C}$ NMR $\left(101 \mathrm{MHz}, \mathrm{CDCl}_{3}\right) \delta$ 203.7, 151.9, 132.6, 131.9, 129.5, 120.2, 117.1, 44.4, 28.6. HRMS (APCI, positive) $\mathrm{m} / z$ calcd for $\mathrm{C}_{10} \mathrm{H}_{14} \mathrm{NO}^{+}[\mathrm{M}+\mathrm{H}]^{+}:$164.1070, found: 164.1068 .

The conversion of $\mathbf{S 5}$ into $\mathbf{1} \mathbf{a}-\boldsymbol{d}_{2}$ was carried out according to the procedure reported. ${ }^{9}$ An oven dried $50 \mathrm{~mL}$ two-neck flask, equipped with a magnetic stirring bar, a condenser with a three-way stopcock, and a rubber septum, was charged with lithium aluminum deuteride (86.0 $\mathrm{mg}, 2.05 \mathrm{mmol})$. The flask was evacuated and backfilled with nitrogen. $\mathrm{Et}_{2} \mathrm{O}(5 \mathrm{~mL})$ was added to the flask, and it was cooled to $-20{ }^{\circ} \mathrm{C}$ by dry ice/acetone bath. Aluminum chloride (TCI, 597 $\mathrm{mg}, 4.48 \mathrm{mmol}$ ) was added to the flask and the mixture was stirred for $20 \mathrm{~min}$ at $-20{ }^{\circ} \mathrm{C} . \mathrm{A} \mathrm{Et}_{2} \mathrm{O}$ (1 mL) solution of S5 (322 mg, $1.97 \mathrm{mmol})$ was added slowly to the flask. The cooling bath was removed, and the mixture was stirred for $22 \mathrm{~h}$ under reflux. The mixture was cooled to $0{ }^{\circ} \mathrm{C}$ by 
ice/water bath, and ethyl acetate $(10 \mathrm{~mL})$ and $\mathrm{H}_{2} \mathrm{SO}_{4}(20 \%$ aqueous solution, $8 \mathrm{~mL})$ were added slowly to the flask. The cooling bath was removed, and sodium hydroxide ( $5 \mathrm{~N}$ aqueous solution) and sodium carbonate was added until the aqueous layer was basic. The organic materials were extracted with $\mathrm{Et}_{2} \mathrm{O}(10 \mathrm{~mL} \times 3)$, washed with brine $(50 \mathrm{~mL} \times 2)$, and dried over anhydrous magnesium sulfate. The crude product was purified by column chromatography on silica gel (eluent: hexane:Et $\left.{ }_{2} \mathrm{O}=97: 3\right)$ and Kugelrhor distillation. 1a-d $\boldsymbol{d}_{2}(155 \mathrm{mg}, 1.02 \mathrm{mmol}, 52 \%$ yield, $>99 \%$ D) was obtained as a colorless oil. 1a- $\boldsymbol{d}_{2}:{ }^{1} \mathrm{H}$ NMR $\left(400 \mathrm{MHz}, \mathrm{CDCl}_{3}\right) \delta 7.25(\mathrm{dd}, J=8.0$, $1.6 \mathrm{~Hz}, 1 \mathrm{H}), 7.19$ (ddd, $J=8.0,7.2,1.6 \mathrm{~Hz}, 1 \mathrm{H}), 7.11$ [dd (AB pattern), $J=8.0,1.6 \mathrm{~Hz}, 1 \mathrm{H}$ ], 7.05 $(\mathrm{td}, J=7.2,1.6 \mathrm{~Hz}, 1 \mathrm{H}), 2.71(\mathrm{~s}, 6 \mathrm{H}), 1.27(\mathrm{~s}, 3 \mathrm{H}) .{ }^{13} \mathrm{C} \mathrm{NMR}\left(101 \mathrm{MHz}, \mathrm{CDCl}_{3}\right) \delta 152.6,138.8$, 129.1, 126.4, 123.4, 119.2, 45.3, 22.9 (quintet, $J=19 \mathrm{~Hz}, C D_{2}$ ), 14.8. HRMS (APCI, posirive) $m / z$ calcd for $\mathrm{C}_{10} \mathrm{H}_{14} \mathrm{D}_{2} \mathrm{~N}^{+}[\mathrm{M}+\mathrm{H}]^{+}:$152.1403, found: 152.1402 .

\section{2-(Ethyl-2,2,2-d $\left.d_{3}\right)-N, N$-dimethylaniline (1a- $\left.d_{3}\right)$}<smiles>CC(=O)c1ccccc1N(C)C</smiles>

S5 pyrrolidine (1.3 equiv)

$\mathrm{D}_{2} \mathrm{O} / 1,4$-dioxane rt, $24 \mathrm{~h}$<smiles>CN(C)c1ccccc1C(=O)O</smiles>

S5- $\boldsymbol{d}_{3}(89 \%, 95 \% \mathrm{D})$

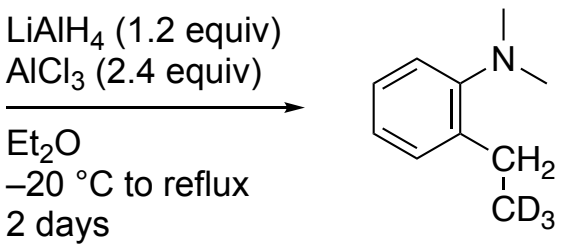

1a-d $_{3}(54 \%, 95 \%$ D)

The conversion of S5 into S5- $\boldsymbol{d}_{3}$ was carried out according to the procedure reported. ${ }^{10}$ An oven dried $100 \mathrm{~mL}$ two-neck flask, equipped with a magnetic stirring bar, a rubber septum, and a three-way stopcock, was evacuated and backfilled with nitrogen. 1,4-Dioxane (4.5 mL), deuterium oxide (Wako, $4.5 \mathrm{~mL})$, pyrrolidine (0.3 mL, $3.7 \mathrm{mmol})$, and $\mathbf{S 5}$ (480 mg, $2.94 \mathrm{mmol})$ were added to the flask. The mixture was stirred for $24 \mathrm{~h}$ at room temperature. The organic materials were extracted with $\mathrm{Et}_{2} \mathrm{O}(20 \mathrm{~mL}$ x 3), washed with brine $(30 \mathrm{~mL} \times 2)$, and dried over anhydrous magnesium sulfate. The crude product was purified by Kugelrhor distillation. S5- $\boldsymbol{d}_{3}$ (435 mg, 2.62 mmol, 89\% yield, 95\% D) was obtained as a yellow liquid. S5- $\boldsymbol{d}_{3}:{ }^{1} \mathrm{H}$ NMR (400 $\left.\mathrm{MHz} \mathrm{CDCl}_{3}\right) \delta 7.43(\mathrm{dd}, J=7.6,1.6 \mathrm{~Hz}, 1 \mathrm{H}), 7.35(\mathrm{ddd}, J=8.4,7.6,1.6 \mathrm{~Hz}, 1 \mathrm{H}), 6.99(\mathrm{~d}, J=$ $8.4 \mathrm{~Hz}, 1 \mathrm{H}), 6.92(\mathrm{td}, J=7.6,0.8 \mathrm{~Hz}, 1 \mathrm{H}), 2.79(\mathrm{~s}, 6 \mathrm{H}) .{ }^{13} \mathrm{C} \mathrm{NMR}\left(101 \mathrm{MHz}, \mathrm{CDCl}_{3}\right) \delta 203.9$, 152.0, 132.6, 132.0, 129.7, 120.3, 117.2, 44.5, 28.0 (septet, $J=20 \mathrm{~Hz}, C_{3}$ ). HRMS (APCI, positive) $m / z$ calcd for $\mathrm{C}_{10} \mathrm{H}_{11} \mathrm{D}_{3} \mathrm{NO}^{+}[\mathrm{M}+\mathrm{H}]^{+}: 167.1258$, found: 167.1257. 
An oven dried $50 \mathrm{~mL}$ two-neck flask, equipped with a magnetic stirring bar, a condenser with a three-way stopcock, and a rubber septum, was charged with lithium aluminum hydride (87.0 $\mathrm{mg}, 2.29 \mathrm{mmol})$. The flask was evacuated and backfilled with nitrogen. $\mathrm{Et}_{2} \mathrm{O}(5 \mathrm{~mL})$ was added to the flask, and it was cooled to $-20^{\circ} \mathrm{C}$ by dry ice/acetone bath. Aluminum chloride (TCI, 640 $\mathrm{mg}, 4.80 \mathrm{mmol}$ ) was added to the flask and the mixture was stirred for $20 \mathrm{~min}$ at $-20{ }^{\circ} \mathrm{C}$. S5- $\boldsymbol{d}_{3}$ (325 mg, $1.96 \mathrm{mmol}$ ) was added slowly to the flask. The cooling bath was removed, and the mixture was stirred for 2 days under reflux. The mixture was cooled to $0{ }^{\circ} \mathrm{C}$ by ice/water bath, and ethyl acetate $(10 \mathrm{~mL})$ and $\mathrm{H}_{2} \mathrm{SO}_{4}(20 \%$ aqueous solution, $8 \mathrm{~mL})$ were added slowly to the flask. The cooling bath was removed, and potassium carbonate was added until the aqueous layer was basic. The organic materials were extracted with $\mathrm{Et}_{2} \mathrm{O}(20 \mathrm{~mL}$ x 3), washed with brine (50 $\mathrm{mL} \times 2$ ), and dried over anhydrous magnesium sulfate. The crude product was purified by column chromatography on silica gel (eluent: hexane: $\mathrm{Et}_{2} \mathrm{O}=97: 3$ ) and Kugelrhor distillation. 1a-d $\boldsymbol{d}_{3}\left(160 \mathrm{mg}, 1.05 \mathrm{mmol}, 54 \%\right.$ yield, 95\% D) was obtained as a colorless oil. 1a- $\boldsymbol{d}_{3}:{ }^{1} \mathrm{H}$ NMR $\left(400 \mathrm{MHz}, \mathrm{CDCl}_{3}\right) \delta 7.24-7.29(\mathrm{~m}, 1 \mathrm{H}), 7.17-7.23(\mathrm{~m}, 1 \mathrm{H}), 7.13$ [dd (AB pattern), $J=8.0,1.6$ $\mathrm{Hz}, 1 \mathrm{H}], 7.07(\mathrm{td}, J=7.2,1.2 \mathrm{~Hz}, 1 \mathrm{H}), 2.77(\mathrm{~s}, 2 \mathrm{H}), 2.73(\mathrm{~s}, 6 \mathrm{H}) .{ }^{13} \mathrm{C} \mathrm{NMR}\left(101 \mathrm{MHz}, \mathrm{CDCl}_{3}\right) \delta$ 152.6, 138.8, 129.0, 126.4, 123.4, 119.2, 45.3, 23.3, 13.6-14.8 (m, $\left.C \mathrm{D}_{3}\right)$. HRMS (APCI, positive) $m / z$ calcd for $\mathrm{C}_{10} \mathrm{H}_{13} \mathrm{D}_{3} \mathrm{~N}^{+}[\mathrm{M}+\mathrm{H}]^{+}:$153.1466, found: 153.1465 .

\section{2-Ethyl- $N, N$-bis(methyl- $\left.d_{3}\right)$ aniline (1a- $\left.d_{6}\right)$}

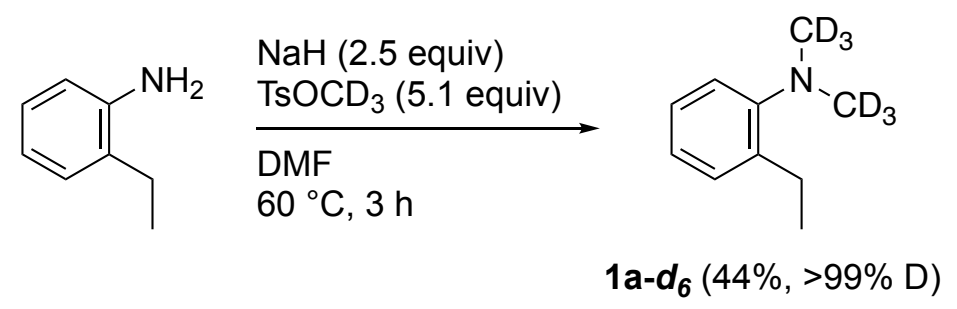

An oven dried $50 \mathrm{~mL}$ two-neck flask, equipped with a magnetic stirring bar, a rubber septum, and a condenser, was charged with $\mathrm{NaH}$ (ca. 60\% dispersion in mineral oil, $0.3 \mathrm{~g}, 7.5 \mathrm{mmol}$ ). The flask was evacuated and backfilled with nitrogen. DMF (9 mL), 2-ethylaniline (Wako, $0.364 \mathrm{~g}$, $3.00 \mathrm{mmol})$, and $\mathrm{TsOCD}_{3}(2.91 \mathrm{~g}, 15.4 \mathrm{mmol})^{11}$ were added to the flask. The mixture was stirred for $3 \mathrm{~h}$ at $60^{\circ} \mathrm{C}$. After cooling to room temperature, hexane $(10 \mathrm{~mL})$, AcOEt $(10 \mathrm{~mL})$, and water $(20 \mathrm{~mL})$ were added to the flask. The organic materials were washed with water $(20 \mathrm{~mL} \times 4)$ and 
brine (20 mL x 1), and dried over anhydrous magnesium sulfate. The crude product was purified by column chromatography on silica gel (SiliaFlash; eluent: hexane: $\mathrm{Et}_{2} \mathrm{O}=97: 3$ ) and Kugelrhor distillation. 1a-d $\boldsymbol{d}_{\boldsymbol{6}}(0.203 \mathrm{~g}, 1.31 \mathrm{mmol}, 44 \%$ yield $)$ was obtained as a colorless oil. $1 \mathbf{1 a}-\boldsymbol{d}_{\boldsymbol{6}}:{ }^{1} \mathrm{H}$ NMR (400 MHz, $\left.\mathrm{CDCl}_{3}\right) \delta 7.25(\mathrm{dd}, J=7.6,1.2 \mathrm{~Hz}, 1 \mathrm{H}), 7.15-7.21(\mathrm{~m}, 1 \mathrm{H}), 7.10$ [dd (AB pattern), $J=7.6,1.2 \mathrm{~Hz}, 1 \mathrm{H}], 7.05(\mathrm{td}, J=7.6,1.2 \mathrm{~Hz}, 1 \mathrm{H}), 2.76(\mathrm{q}, J=7.6 \mathrm{~Hz}, 2 \mathrm{H}), 1.28(\mathrm{t}, J=$ 7.6 Hz, 3H). ${ }^{13} \mathrm{C} \mathrm{NMR}\left(101 \mathrm{MHz}, \mathrm{CDCl}_{3}\right) \delta 152.6,138.8,129.0,126.4,123.4,119.1,44.3$ (septet, $\left.J=20 \mathrm{~Hz}, C \mathrm{D}_{3}\right), 23.6,14.9$. HRMS $(\mathrm{APCI}) \mathrm{m} / z$ calcd for $\mathrm{C}_{10} \mathrm{H}_{10} \mathrm{D}_{6} \mathrm{~N}^{+}[\mathrm{M}+\mathrm{H}]^{+}: 156.1654$, found: 156.1652 .

\section{7-2. Reaction of D-Labeled Substrates (Figure 2)}

\section{The Reaction of $1 \mathrm{a}-d_{2}$ (entry 1)}

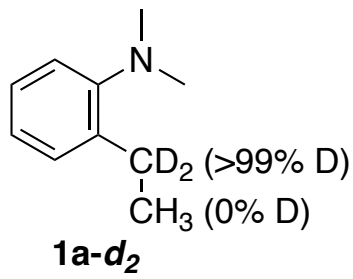

\begin{tabular}{l}
{$\left[\mathrm{IrCl}\left(\mathrm{C}_{2} \mathrm{H}_{4}\right)_{2}\right]_{2}$} \\
$\mathrm{~L} 3(6 \mathrm{~mol} \%)$ \\
\hline $\operatorname{TBE}(2$ equiv) \\
mesitylene \\
$150{ }^{\circ} \mathrm{C}, 24 \mathrm{~h}$
\end{tabular}

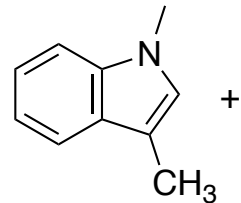

$2 a$

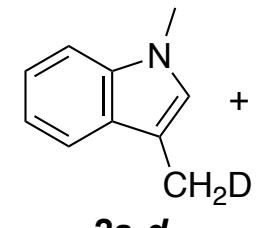

2a-d $80 \%(50: 46: 4)$

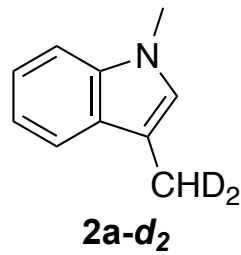

$2 \mathrm{a}-\mathrm{d}_{2}$

According to the general procedure $A$ described in section 5, the reaction was carried out at $150{ }^{\circ} \mathrm{C}$ for $24 \mathrm{~h}$ using $\left[\operatorname{IrCl}\left(\mathrm{C}_{2} \mathrm{H}_{4}\right)_{2}\right]_{2}(3.41 \mathrm{mg}, 0.00601 \mathrm{mmol}), \mathbf{L 3}$ (14.0 mg, $\left.0.0119 \mathrm{mmol}\right)$, 1a-d 2 (31.1 mg, $0.206 \mathrm{mmol})$, tert-butylethylene (34.2 $\mathrm{mg}, 0.406 \mathrm{mmol})$, and mesitylene $(0.4$ $\mathrm{mL})$. A mixture of $\mathbf{2 a}$ and $\mathbf{2 a}-\boldsymbol{d}_{\boldsymbol{1}}(24.0 \mathrm{mg}, 0.164 \mathrm{mmol}, 80 \%)$ was obtained as a white solid after purification by column chromatography on silica gel (eluent: hexane: $\mathrm{Et}_{2} \mathrm{O}: \mathrm{Et}_{3} \mathrm{~N}=45: 1: 1$ ). $\mathbf{2 a}$ and

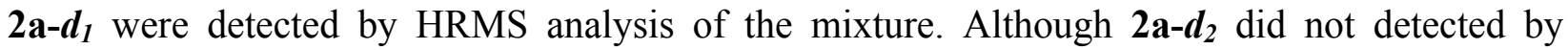
HRMS analysis, ${ }^{1} \mathrm{H}$ NMR analysis indicated formation of it. The ratio of $\mathbf{2} \mathbf{a}, \mathbf{2} \mathbf{a}-\boldsymbol{d}_{\mathbf{1}}$, and $\mathbf{2} \mathbf{a}-\boldsymbol{d}_{2}$ was determined as 50:46:4 by ${ }^{2} \mathrm{H}$-decoupled ${ }^{1} \mathrm{H}$ NMR measurement. This ratio did not change after 48 $\mathrm{h}$ under the reaction conditions. Followings are the peaks observed in NMR analyses of a mixture of 2a, 2a-d $\mathbf{d}_{1}$, and $\mathbf{2 a - d _ { 2 } :}{ }^{1} \mathrm{H}$ NMR $\left(400 \mathrm{MHz}, \mathrm{CDCl}_{3}\right) \delta 7.61(\mathrm{~d}, J=8.0 \mathrm{~Hz}, 1 \mathrm{H}), 7.31[\mathrm{~d}(\mathrm{AB}$ pattern), $J=8.0 \mathrm{~Hz}, 1 \mathrm{H}], 7.26(\mathrm{t}, J=8.0 \mathrm{~Hz}, 1 \mathrm{H}), 7.15$ (t, $J=8.0 \mathrm{~Hz}, 1 \mathrm{H}), 6.85(\mathrm{~s}, 1 \mathrm{H}), 3.76$ (s, 3H), 2.34-2.39 (m, 2.45H). ${ }^{13} \mathrm{C}$ NMR (101 MHz, $\left.\mathrm{CDCl}_{3}\right) \delta 137.1,128.8,126.6,121.5,119.0$, 118.6, 110.2, 109.1, 32.6, $9.7\left(\mathrm{CH}_{3}\right), 9.4$ (t, $\left.J=20 \mathrm{~Hz}, \mathrm{CH}_{2} \mathrm{D}\right) .2 \mathrm{2a}$ : HRMS (APCI, positive) $m / z$ 


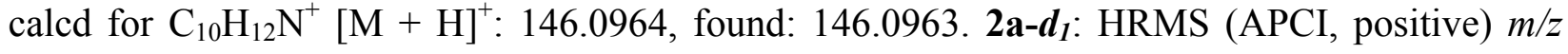
calcd for $\mathrm{C}_{10} \mathrm{H}_{11} \mathrm{DN}^{+}[\mathrm{M}+\mathrm{H}]^{+}:$147.1027, found: 147.1022 .

\section{The Reaction of $1 \mathrm{a}-d_{3}$ (entry 2 )}

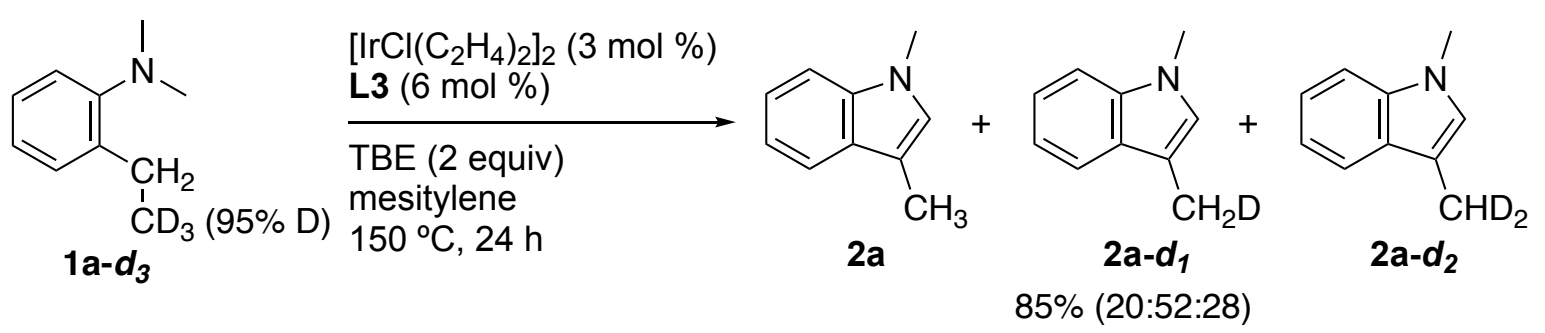

According to the general procedure $A$ described in section 5 , the reaction was carried out at $150{ }^{\circ} \mathrm{C}$ for $24 \mathrm{~h}$ using $\left[\operatorname{IrCl}\left(\mathrm{C}_{2} \mathrm{H}_{4}\right)_{2}\right]_{2}(4.01 \mathrm{mg}, 0.00707 \mathrm{mmol}), \mathbf{L 3}$ (15.3 mg, $\left.0.0130 \mathrm{mmol}\right)$, 1a-d 3 (28.7 mg, $0.188 \mathrm{mmol})$, tert-butylethylene $(32.8 \mathrm{mg}, 0.390 \mathrm{mmol})$, and mesitylene $(0.4$ $\mathrm{mL})$. A mixture of $\mathbf{2 a}, \mathbf{2 a}-\boldsymbol{d}_{1}$, and $\mathbf{2 a -} \boldsymbol{d}_{2}(24.4 \mathrm{mg}, 0.160 \mathrm{mmol}, 85 \%)$ was obtained as a white solid after purification by column chromatography on silica gel (eluent: hexane: $\mathrm{Et}_{2} \mathrm{O}: \mathrm{Et}_{3} \mathrm{~N}=$ 45:1:1). 2a, 2a- $\boldsymbol{d}_{1}$, and $\mathbf{2 a}-\boldsymbol{d}_{2}$ were detected by HRMS analysis of the mixture. The ratio of $\mathbf{2 a}$, 2a- $\boldsymbol{d}_{1}$, and $\mathbf{2 a - \boldsymbol { d } _ { 2 }}$ was determined as 20:52:28 by ${ }^{2} \mathrm{H}$-decoupled ${ }^{1} \mathrm{H}$ NMR measurement. This ratio did not change after $48 \mathrm{~h}$ under the reaction conditions. Followings are the peaks observed in

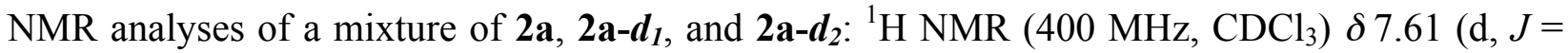
$7.6 \mathrm{~Hz}, 1 \mathrm{H}), 7.31$ [d (AB pattern), $J=7.6 \mathrm{~Hz}, 1 \mathrm{H}], 7.26(\mathrm{t}, J=7.6 \mathrm{~Hz}, 1 \mathrm{H}), 7.14$ (t, $J=7.6 \mathrm{~Hz}$, $1 \mathrm{H}), 6.85(\mathrm{~s}, 1 \mathrm{H}), 3.75(\mathrm{~s}, 3 \mathrm{H}), 2.31-2.39(\mathrm{~m}, 1.91 \mathrm{H}) .{ }^{13} \mathrm{C} \mathrm{NMR}\left(101 \mathrm{MHz}, \mathrm{CDCl}_{3}\right) \delta 137.1$, 128.8, 126.6, 121.5, 119.1, 118.6, 110.2, 109.1, 32.6, $9.7\left(\mathrm{CH}_{3}\right), 9.4$ (t, $\left.J=20 \mathrm{~Hz}, \mathrm{CH}_{2} \mathrm{D}\right), 9.2$ (quintet, $J=20 \mathrm{~Hz}, C \mathrm{HD}_{2}$ ). 2a: HRMS (APCI, positive) $m / z$ calcd for $\mathrm{C}_{10} \mathrm{H}_{12} \mathrm{~N}^{+}[\mathrm{M}+\mathrm{H}]^{+}$: 146.0964, found: 146.0966. 2a- $\boldsymbol{d}_{1}$ : HRMS (APCI, positive) $m / z$ calcd for $\mathrm{C}_{10} \mathrm{H}_{11} \mathrm{DN}^{+}[\mathrm{M}+\mathrm{H}]^{+}$: 147.1027, found: 147.1027. 2a- $\boldsymbol{d}_{2}$ : HRMS (APCI, positive) $m / z$ calcd for $\mathrm{C}_{10} \mathrm{H}_{10} \mathrm{D}_{2} \mathrm{~N}^{+}[\mathrm{M}+\mathrm{H}]^{+}$: 148.1090, found: 148.1088 . 
The Reaction of $1 \mathrm{a}-d_{6}$ (ref. 22 in the text)

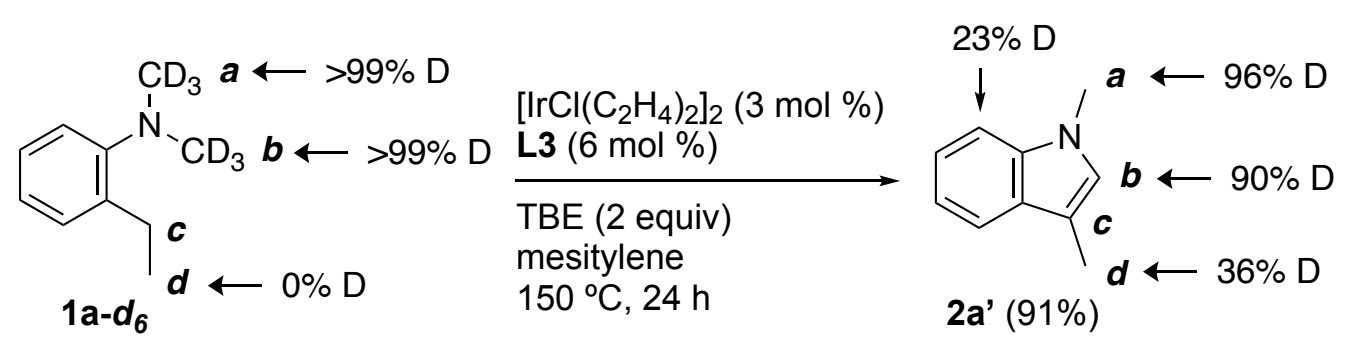

According to the general procedure $A$ described in section 5 , the reaction was carried out at $150{ }^{\circ} \mathrm{C}$ for $24 \mathrm{~h}$ using $\left[\operatorname{IrCl}\left(\mathrm{C}_{2} \mathrm{H}_{4}\right)_{2}\right]_{2}(4.48 \mathrm{mg}, 0.00789 \mathrm{mmol}), \mathbf{L 3}$ (15.1 mg, $\left.0.0128 \mathrm{mmol}\right)$, 1a-d $\boldsymbol{d}_{\mathbf{6}}(28.7 \mathrm{mg}, 0.185 \mathrm{mmol})$, tert-butylethylene $(34.9 \mathrm{mg}, 0.415 \mathrm{mmol})$, and mesitylene $(0.4$ $\mathrm{mL}$ ). 2a' (25.5 mg, $0.169 \mathrm{mmol}, 91 \%$ ) was obtained as a white solid after purification by column chromatography on silica gel (eluent: hexane: $\mathrm{Et}_{2} \mathrm{O}: \mathrm{Et}_{3} \mathrm{~N}=45: 1: 1$ ). ${ }^{1} \mathrm{H} \mathrm{NMR}$ analysis of $\mathbf{2 a}$ ' indicated that $36 \%$ of $\mathrm{H}$ on $\mathrm{C}^{d}$ was replaced by $\mathrm{D}$. The exact mass for $\mathrm{C}_{10} \mathrm{H}_{6} \mathrm{D}_{5} \mathrm{~N}\left(\boldsymbol{d}_{5}\right)$ and $\mathrm{C}_{10} \mathrm{H}_{5} \mathrm{D}_{6} \mathrm{~N}\left(\boldsymbol{d}_{\boldsymbol{\sigma}}\right)$ were detected by HRMS analysis of 2a'. 2a': ${ }^{1} \mathrm{H}$ NMR (400 MHz, $\left.\mathrm{CDCl}_{3}\right) \delta 7.62$ (dt, $J=8.0,1.2 \mathrm{~Hz}, 1.00 \mathrm{H}), 7.32$ [dt (AB pattern), $J=8.4,1.2 \mathrm{~Hz}, 0.77 \mathrm{H}], 7.24-7.29$ (m, 1.00H), 7.13-7.18 (m, 1.00H), $6.85(\mathrm{~s}, 0.10 \mathrm{H}), 3.71-3.76(\mathrm{~m}, 0.11 \mathrm{H}), 2.32-2.38(\mathrm{~m}, 1.91 \mathrm{H}) .{ }^{13} \mathrm{C}$ NMR $\left(101 \mathrm{MHz}, \mathrm{CDCl}_{3}\right) \delta 137.1,128.8,126.6,121.5,121.4,119.0,118.6,110.2,110.0,109.1$, 31.2-32.2 (m), 30.5, 8.89-9.8 (m). HRMS (APCI, positive) $m / z$ calcd for $\mathrm{C}_{10} \mathrm{H}_{7} \mathrm{D}_{5} \mathrm{~N}^{+}[\mathrm{M}+\mathrm{H}]^{+}$: 151.1278, found: $151.1277 ; \mathrm{m} / z$ calcd for $\mathrm{C}_{10} \mathrm{H}_{6} \mathrm{D}_{6} \mathrm{~N}^{+}[\mathrm{M}+\mathrm{H}]^{+}:$152.1341, found: 152.1338 .

\section{7-3. Discussion for the H/D Scrambling}

Assuming that H/D scrambling through elimination-addition of $\mathrm{H}-\mathrm{Ir}-\mathrm{D}$ does not occur, the reaction pathways for the conversion of $\mathbf{1 a - d _ { 2 }}$ are proposed as shown in Scheme S1. Based on the

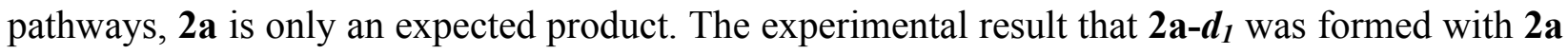
reasonably explained by existence of fast equilibrium between $\mathbf{A}$ and $\mathbf{B}$ and slow ligand exchange of $\mathbf{B}$ with tert-butylethylene to give $\mathbf{C}$ and $\mathbf{3}$ (Scheme 4). 


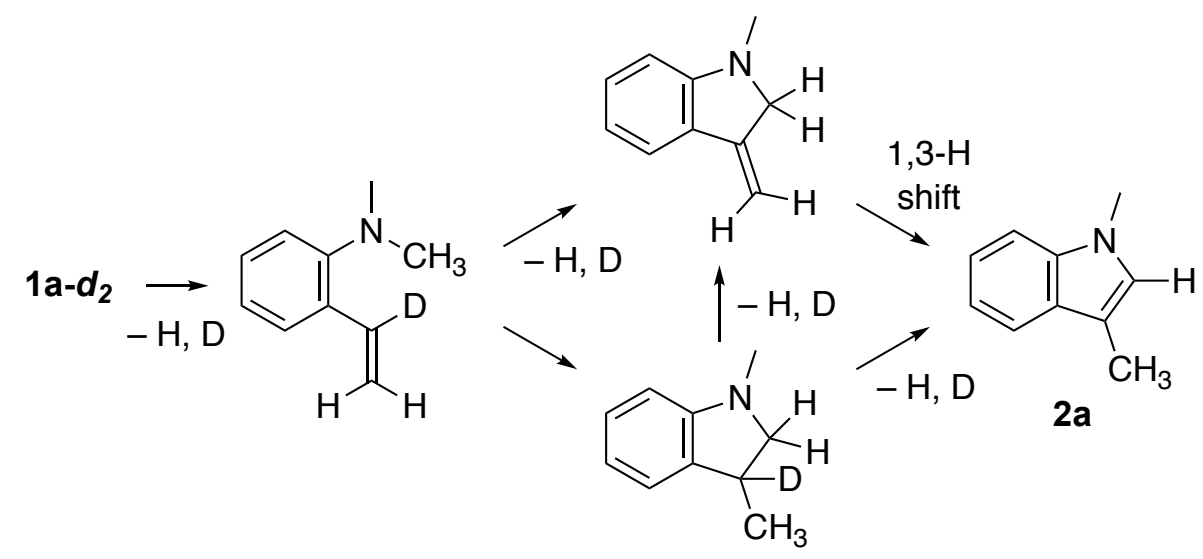

Scheme S1. Possible Reaction Pathways of $\mathbf{1 a}_{\mathbf{2}} \boldsymbol{d}_{\mathbf{2}}$ Assuming no H/D Scrambling through Elimination-Addition of $\mathrm{H}-\mathrm{Ir}-\mathrm{D}$

Under the same assumption, the reaction pathways for the conversion of $\mathbf{1 a}-\boldsymbol{d}_{3}$ are proposed as shown in Scheme S2. Based on the pathways, 2a- $\boldsymbol{d}_{1}$ and $\mathbf{2 a - \boldsymbol { d } _ { 2 }}$ are expected products. The experimental result that $\mathbf{2 a}$ was formed with $\mathbf{2 a}-\boldsymbol{d}_{\mathbf{1}}$ and $\mathbf{2 a - \boldsymbol { d } _ { 2 }}$ also reasonably explained by existence of fast equilibrium between $\mathbf{A}$ and $\mathbf{B}$ and slow ligand exchange of $\mathbf{B}$ with tert-butylethylene to give $\mathbf{C}$ and $\mathbf{3}$ (Scheme 4).

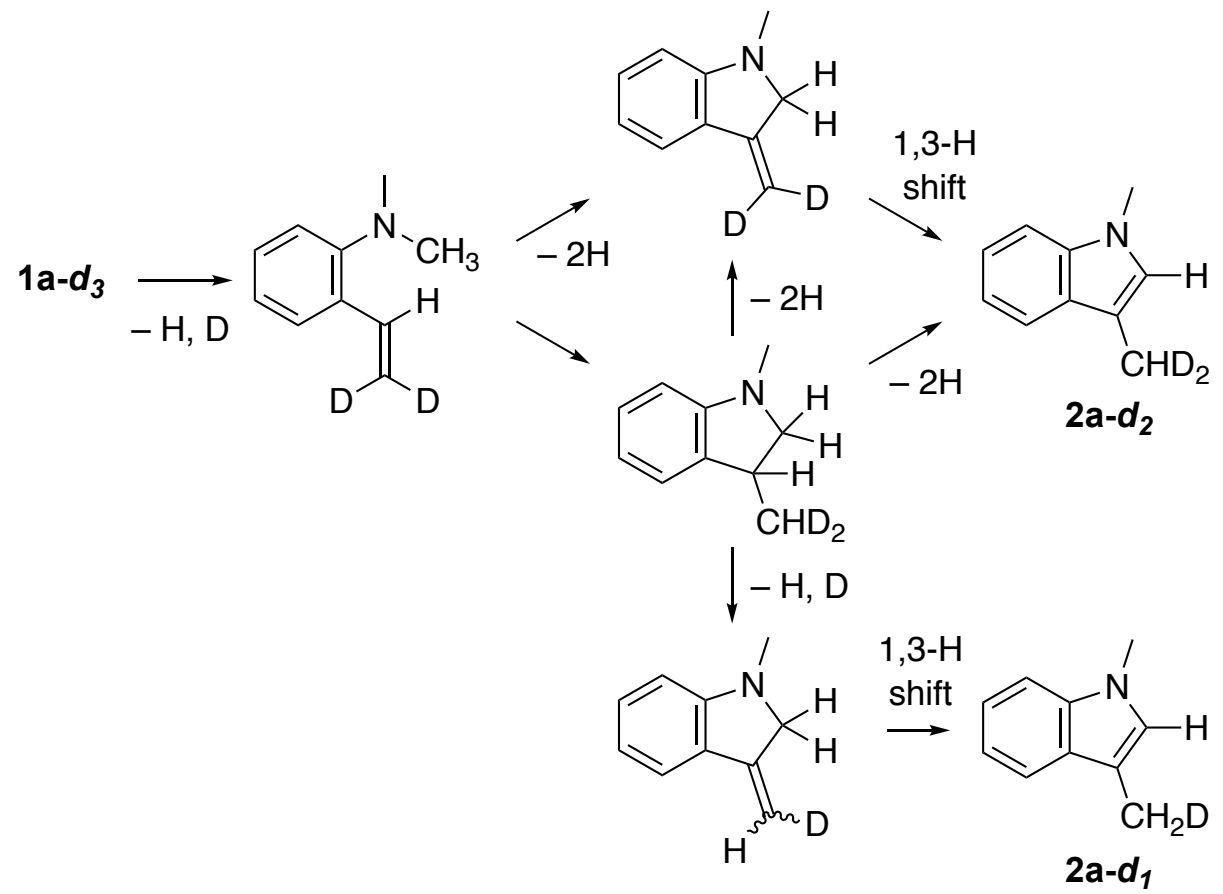

Scheme S2 Possible Reaction Pathways of 1a- $\boldsymbol{d}_{3}$ Assuming no H/D Scrambling through Elimination-Addition of $\mathrm{H}-\mathrm{Ir}-\mathrm{D}$ 
Under the same assumption, the reaction pathways for the conversion of $\mathbf{1 a - d _ { 6 }}$ are proposed as shown in Scheme S3. Based on the pathways, the percentage of D at the $\mathrm{C}^{d}$ of the product is expected to be $33-67 \%$. The experimental result $(36 \% \mathrm{D})$ is within this range.

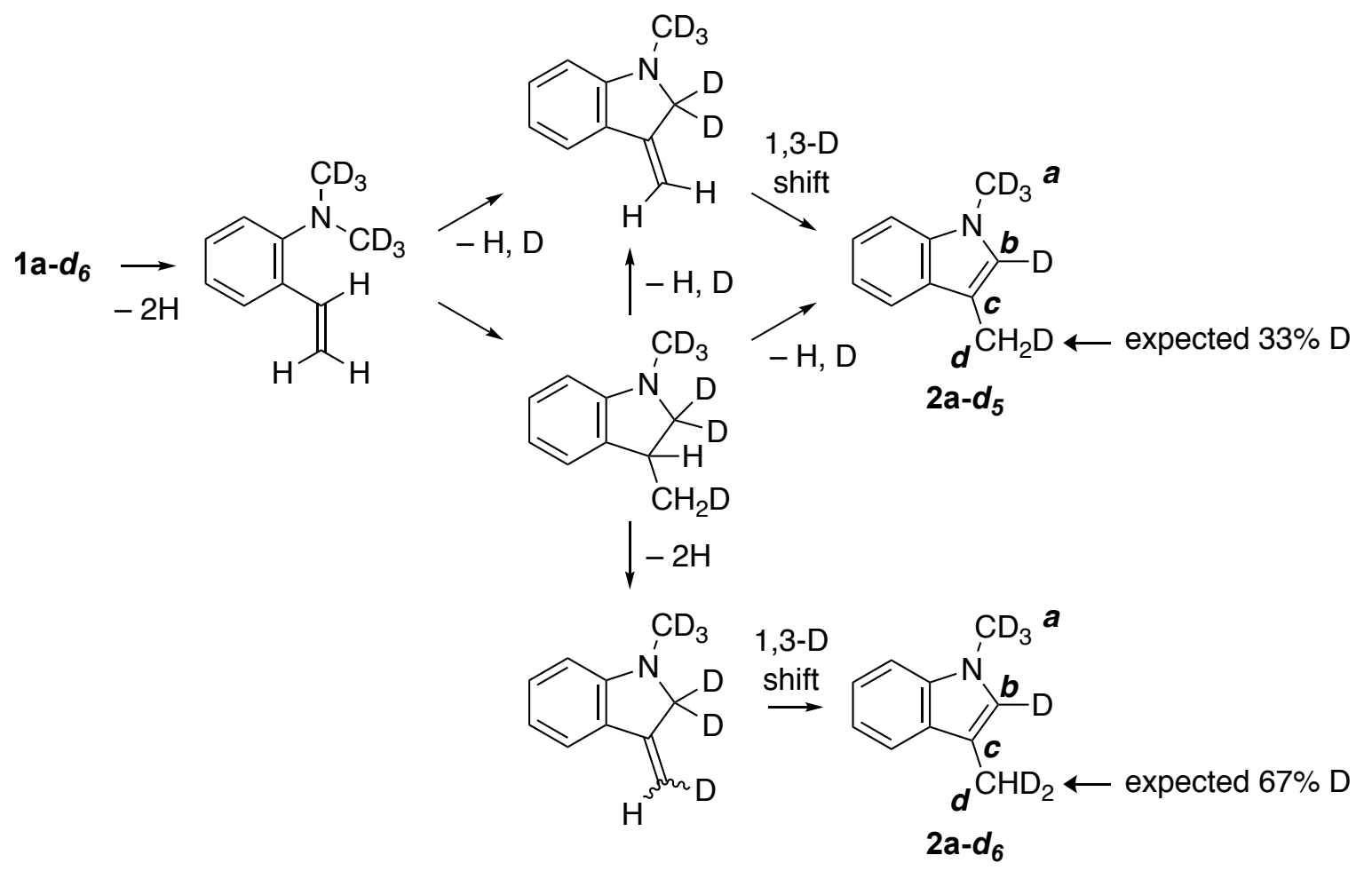

Scheme S3 Possible Reaction Pathways of 1a-d $\boldsymbol{d}_{6}$ Assuming no H/D Scrambling through Elimination-Addition of $\mathrm{H}-\mathrm{Ir}-\mathrm{D}$

\section{Application for the Isomer-Selective Synthesis of Borylindoles (Scheme 4-i)}

\section{8-1. Synthesis of 1,3,7-Trimethyl-2-(4,4,5,5-tetramethyl-1,3,2-dioxaborolan-2-yl)-1H-indole} (13)

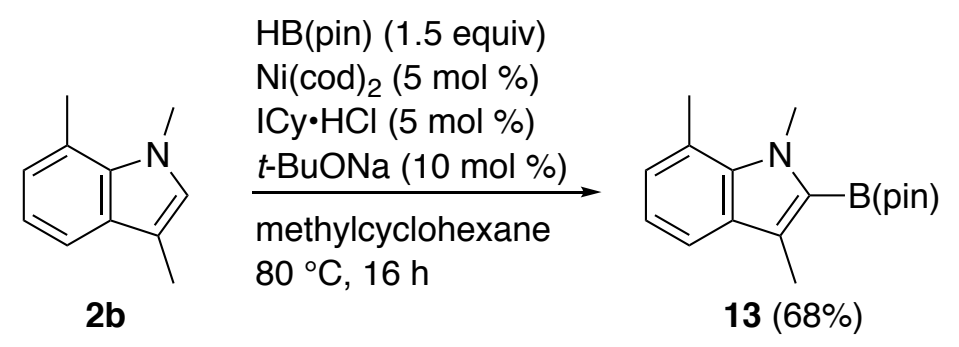

The nickel-catalyzed C-H borylation was carried out according to the procedure reported. ${ }^{12}$ In a glovebox, a glass tube (outside diameter: $20 \mathrm{~mm}$ ) having PTFE stopcock (J. Young), equipped with a magnetic stirring bar, was charged with $\mathrm{Ni}(\mathrm{cod})_{2}(1.28 \mathrm{mg}, 0.00465 \mathrm{mmol}), \mathrm{ICy} \cdot \mathrm{HCl}(1.28$ mg, $0.00476 \mathrm{mmol}), t$-BuONa (0.97 mg, $0.0101 \mathrm{mmol}), \mathrm{HB}($ pin) $(17.5 \mathrm{mg}, 0.137 \mathrm{mmol}), 2 \mathbf{b}$ 
(14.9 $\mathrm{mg}, 0.0936 \mathrm{mmol})$, and methylcyclohexane $(0.125 \mathrm{~mL})$. The tube was sealed by the stopcock and was taken out from the glovebox. The mixture was stirred at $80{ }^{\circ} \mathrm{C}$ by a heating magnetic stirrer with an aluminum heating block (hole size: $21 \mathrm{~mm}$ diameter x $33 \mathrm{~mm}$ depth). After $16 \mathrm{~h}$, the tube was cooled to room temperature, and the volatiles were removed under reduced pressure. $13(18.2 \mathrm{mg}, 0.0638 \mathrm{mmol}, 68 \%)$ was obtained as a white solid after purification by column chromatography on silica gel (eluent: hexane:Et ${ }_{2} \mathrm{O}=20: 1$ ). 13: ${ }^{1} \mathrm{H} \mathrm{NMR}$ $\left(400 \mathrm{MHz}, \mathrm{CDCl}_{3}\right) \delta$ 7.41-7.47 (m, 1H), 6.91-6.97 (m, 2H), $4.22(\mathrm{~s}, 3 \mathrm{H}), 2.79(\mathrm{~s}, 3 \mathrm{H}), 2.53(\mathrm{~s}$, 3H), $1.38(\mathrm{~s}, 12 \mathrm{H}) .{ }^{13} \mathrm{C} \mathrm{NMR}\left(101 \mathrm{MHz}, \mathrm{CDCl}_{3}\right) \delta 139.1,129.6,126.3,125.1,121.5,118.8$, 118.0, 83.3, 35.6, 25.0, 20.8, 10.4. The boron-bound carbon was not detected due to quadrupolar relaxation. ${ }^{11} \mathrm{~B} \mathrm{NMR}\left(101 \mathrm{MHz}, \mathrm{CDCl}_{3}\right) \delta$ 28.6. HRMS (APCI, positive) $\mathrm{m} / \mathrm{z}$ calcd for $\mathrm{C}_{17} \mathrm{H}_{25} \mathrm{BNO}_{2}^{+}[\mathrm{M}+\mathrm{H}]^{+}: 286.1973$, found: 286.1978 .

\section{8-2. Synthesis of 1,3,7-Trimethyl-5-(4,4,5,5-tetramethyl-1,3,2-dioxaborolan-2-yl)-1H-indole}

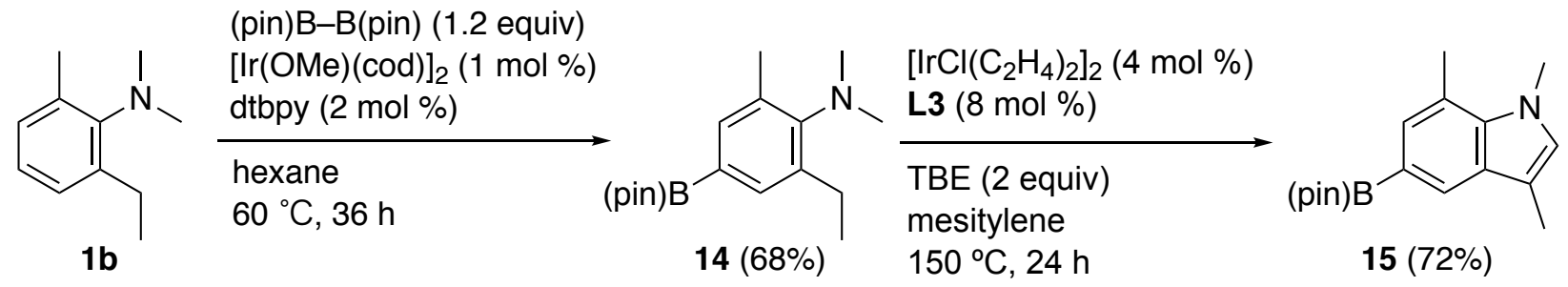

The iridium-catalyzed $\mathrm{C}-\mathrm{H}$ borylation was carried out according to the procedure reported. ${ }^{13}$ In a glovebox, a glass tube (outside diameter: $20 \mathrm{~mm}$ ) having PTFE stopcock (J. Young), equipped with a magnetic stirring bar, was charged with $[\operatorname{Ir}(\mathrm{OMe})(\mathrm{cod})]_{2}(26.1 \mathrm{mg}, 0.0394$ mmol), 4,4'-di-tert-butyl-2,2'-bipyridine (dtbpy, $22.4 \mathrm{mg}, 0.0835 \mathrm{mmol}$ ), bis(pinacolato)diboron (1.20 g, $4.73 \mathrm{mmol})$, hexane $(6 \mathrm{~mL})$, and $1 \mathbf{b}(0.634 \mathrm{~g}, 3.88 \mathrm{mmol})$. The tube was sealed by the stopcock and was taken out from the glovebox. The mixture was stirred at $60{ }^{\circ} \mathrm{C}$ by a heating magnetic stirrer with an aluminum heating block (hole size: $21 \mathrm{~mm}$ diameter x $33 \mathrm{~mm}$ depth). After $36 \mathrm{~h}$, the tube was cooled to room temperature, and the volatiles were removed under reduced pressure. $14(0.762 \mathrm{~g}, 2.63 \mathrm{mmol}, 68 \%)$ was obtained as a white solid after purification by column chromatography on silica gel (eluent: hexane: $\mathrm{Et}_{2} \mathrm{O}=20: 1$ ) and Kugelrhor distillation. 14: ${ }^{1} \mathrm{H}$ NMR (400 MHz, $\left.\mathrm{CDCl}_{3}\right) \delta 7.59(\mathrm{~s}, 1 \mathrm{H}), 7.55$ (s, 1H), $2.90(\mathrm{~s}, 6 \mathrm{H}), 2.75$ (q, J= 7.6 Hz, 
2H), $2.38(\mathrm{~s}, 3 \mathrm{H}), 1.40(\mathrm{~s}, 12 \mathrm{H}), 1.30(\mathrm{t}, J=7.6 \mathrm{~Hz}, 3 \mathrm{H}) .{ }^{13} \mathrm{C} \mathrm{NMR}\left(101 \mathrm{MHz}, \mathrm{CDCl}_{3}\right) \delta 152.5$, 142.8, 136.7, 135.8, 134.0, 125.3 (broad, $C-\mathrm{B}), 83.6,42.9,25.3,24.9,19.2,15.8 .{ }^{11} \mathrm{~B}$ NMR $(128$ $\left.\mathrm{MHz}, \mathrm{CDCl}_{3}\right) \delta 30.4$. HRMS (APCI, positive) $m / z$ calcd for $\mathrm{C}_{17} \mathrm{H}_{29} \mathrm{BNO}_{2}^{+}[\mathrm{M}+\mathrm{H}]^{+}: 290.2286$, found: 290.2288 .

According to the general procedure $A$ described in section 5 , the reaction was carried out at $150{ }^{\circ} \mathrm{C}$ for $24 \mathrm{~h}$ using $\left[\mathrm{IrCl}\left(\mathrm{C}_{2} \mathrm{H}_{4}\right)_{2}\right]_{2}(4.63 \mathrm{mg}, 0.00816 \mathrm{mmol}), \mathbf{L 3}(17.7 \mathrm{mg}, 0.0150 \mathrm{mmol}), 14$ (57.9 mg, $0.200 \mathrm{mmol})$, tert-butylethylene $(32.3 \mathrm{mg}, 0.384 \mathrm{mmol})$, and mesitylene $(0.4 \mathrm{~mL}) .15$ (40.8 $\mathrm{mg}, 0.143 \mathrm{mmol}, 72 \%$ including $<2 \%$ of a structural isomer) was obtained as a white solid after purification by column chromatography on silica gel (eluent: toluene: $\mathrm{Et}_{3} \mathrm{~N}=30: 1$ ). 15: ${ }^{1} \mathrm{H}$ NMR (400 MHz, $\left.\mathrm{CDCl}_{3}\right) \delta 7.92(\mathrm{~s}, 1 \mathrm{H}), 7.35(\mathrm{~s}, 1 \mathrm{H}), 6.69(\mathrm{~d}, J=1.2 \mathrm{~Hz}, 1 \mathrm{H}), 3.98(\mathrm{~s}, 3 \mathrm{H}), 2.75$ (s, 3H), $2.30(\mathrm{~d}, J=1.2 \mathrm{~Hz}, 3 \mathrm{H}), 1.38(\mathrm{~s}, 12 \mathrm{H}) .{ }^{13} \mathrm{C} \mathrm{NMR}\left(101 \mathrm{MHz}, \mathrm{CDCl}_{3}\right) \delta$ 137.8, 130.0, $129.5,128.2,125.1,120.2,110.8,83.3,36.3,24.9,19.5,9.6$. The boron-bound carbon was not detected due to quadrupolar relaxation. ${ }^{11} \mathrm{~B} \mathrm{NMR}\left(128 \mathrm{MHz}, \mathrm{CDCl}_{3}\right) \delta 30.7$. HRMS (APCI, positive) $m / z$ calcd for $\mathrm{C}_{17} \mathrm{H}_{25} \mathrm{BNO}_{2}^{+}[\mathrm{M}+\mathrm{H}]^{+}: 286.1973$, found: 286.1977 .

\section{Application for the Isomer-Selective Synthesis of Bromoindoles (Scheme 4-ii) \\ 9-1. Synthesis of 2-Bromo-7-ethyl-1,3-dimethyl-1H-indole (16)}

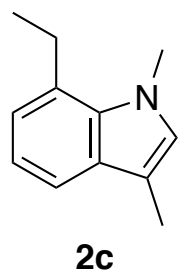

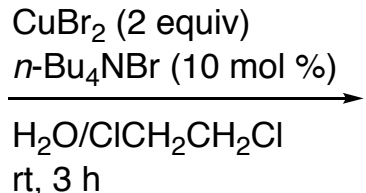

$\mathrm{rt}, 3 \mathrm{~h}$

2c

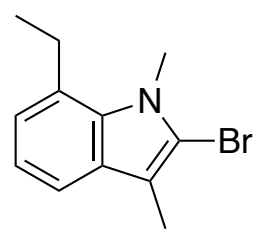

$16(78 \%)$

The bromination using $\mathrm{CuBr}_{2}$ and $n-\mathrm{Bu}_{4} \mathrm{NBr}$ was carried out according to the procedure reported. ${ }^{14}$ In a glovebox, a glass tube (outside diameter: $20 \mathrm{~mm}$ ) having PTFE stopcock (J. Young), equipped with a magnetic stirring bar, was charged with $\mathrm{CuBr}_{2}$ (97.4 mg, $0.436 \mathrm{mmol}$ ), tetrabutylammonium bromide $(7.56 \mathrm{mg}, 0.0235 \mathrm{mmol}), \mathbf{2 c}(36.3 \mathrm{mg}, 0.210 \mathrm{mmol})$, and 1,2-dichloroethane $(2 \mathrm{~mL})$. The tube was sealed by the stopcock and was taken out from the glovebox. Water $(0.080 \mathrm{~mL})$ was added to the tube under the nitrogen stream, and the tube was sealed by the stopcock again. The mixture was stirred for $3 \mathrm{~h}$ at room temperature. 16 (41.3 $\mathrm{mg}$, $0.164 \mathrm{mmol}, 78 \%$ ) was obtained as a white solid after purification by column chromatography on 
silica gel (eluent: hexane:Et $\left.{ }_{2} \mathrm{O}=80: 1\right) .16:{ }^{1} \mathrm{H}$ NMR $\left(400 \mathrm{MHz}, \mathrm{CDCl}_{3}\right) \delta 7.38(\mathrm{dd}, J=7.6,1.2$ $\mathrm{Hz}, 1 \mathrm{H}), 7.06$ (t, $J=7.6 \mathrm{~Hz}, 1 \mathrm{H}), 6.98-7.02(\mathrm{~m}, 1 \mathrm{H}), 4.00(\mathrm{~s}, 3 \mathrm{H}), 3.12$ (q, $J=7.6 \mathrm{~Hz}, 2 \mathrm{H}), 2.31$ $(\mathrm{s}, 3 \mathrm{H}), 1.37(\mathrm{t}, J=7.6 \mathrm{~Hz}, 3 \mathrm{H}) .{ }^{13} \mathrm{C} \mathrm{NMR}\left(101 \mathrm{MHz}, \mathrm{CDCl}_{3}\right) \delta 135.3,129.0,127.4,123.2,119.8$, 116.4, 114.8, 111.0, 34.6, 26.1, 16.7, 10.4. HRMS (APCI, positive) $m / z$ calcd for $\mathrm{C}_{12} \mathrm{H}_{15} \mathrm{BrN}^{+}[\mathrm{M}$ $+\mathrm{H}]^{+}:$252.0382, found: 252.0384 .

\section{9-2. Synthesis of 6-Bromo-7-ethyl-1,3-dimethyl-1H-indole (19)}

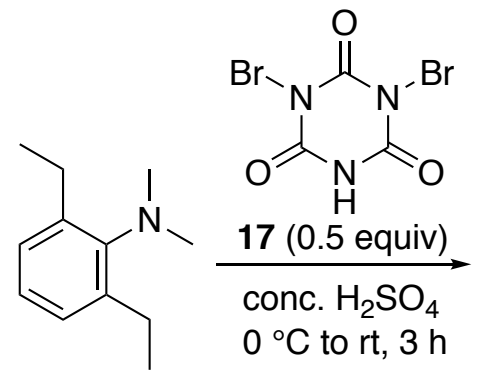

$1 \mathrm{c}$

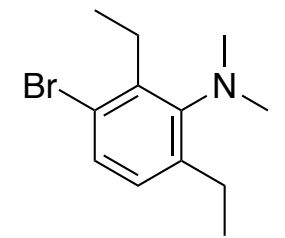

$18(81 \%)$

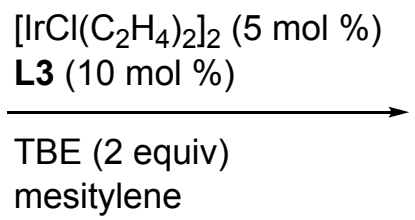

$150{ }^{\circ} \mathrm{C}, 24 \mathrm{~h}$

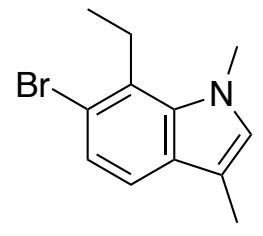

$19(75 \%)$

The bromination using dibromoisocyanuric acid (17) was carried out according to the procedure reported. $^{15}$ A $100 \mathrm{~mL}$ three-neck flask, equipped with a magnetic stirring bar, a dropping funnel, a rubber septum, and a three-way stopcock, was evacuated and backfilled with nitrogen. 1c $(0.340 \mathrm{~g}, 1.92 \mathrm{mmol})$ and conc. $\mathrm{H}_{2} \mathrm{SO}_{4}(2 \mathrm{~mL})$ were added to the flask, and the mixture was cooled to $0{ }^{\circ} \mathrm{C}$ by ice/water bath. A suspension of 17 (0.296 g, $\left.1.03 \mathrm{mmol}\right)$ in conc. $\mathrm{H}_{2} \mathrm{SO}_{4}(4 \mathrm{~mL})$ was added slowly to the flask through the dropping funnel. The cooling bath was removed, and the mixture was stirred for $3 \mathrm{~h}$ at room temperature. Ice was added to the flask, and then potassium carbonate were added until the aqueous layer was basic. The organic materials were extracted with $\mathrm{Et}_{2} \mathrm{O}(60 \mathrm{~mL} \times 3)$, washed with brine $(100 \mathrm{~mL} \times 1)$, and dried over anhydrous magnesium sulfate. The crude product was purified by column chromatography on silica gel (eluent: hexane) and Kugelrhor distillation. 18 (0.396 g, $1.55 \mathrm{mmol}, 81 \%)$ was obtained as a colorless oil. 18: ${ }^{1} \mathrm{H}$ NMR $\left(400 \mathrm{MHz}, \mathrm{CDCl}_{3}\right) \delta 7.33(\mathrm{~d}, J=8.0 \mathrm{~Hz}, 1 \mathrm{H}), 6.91(\mathrm{~d}, J=8.0 \mathrm{~Hz}$, 1H), $2.84(\mathrm{~s}, 6 \mathrm{H}), 2.83(\mathrm{q}, J=7.6 \mathrm{~Hz}, 2 \mathrm{H}), 2.61$ (q, $J=7.6 \mathrm{~Hz}, 2 \mathrm{H}), 1.21(\mathrm{t}, J=7.6 \mathrm{~Hz}, 3 \mathrm{H}), 1.20$ $(\mathrm{t}, J=7.6 \mathrm{~Hz}, 3 \mathrm{H}) .{ }^{13} \mathrm{C} \mathrm{NMR}\left(101 \mathrm{MHz}, \mathrm{CDCl}_{3}\right) \delta 150.5,144.2,144.1,130.5,128.2,122.4,44.0$, 25.8, 24.8, 15.5, 14.4. HRMS (APCI, positive) $m / z$ calcd for $\mathrm{C}_{12} \mathrm{H}_{18} \mathrm{BrN}^{+}[\mathrm{M}+\mathrm{H}]^{+}: 256.0695$, found: 256.0697 . 
According to the general procedure $A$ described in section 5, the reaction was carried out at $150{ }^{\circ} \mathrm{C}$ for $24 \mathrm{~h}$ using $\left[\mathrm{IrCl}\left(\mathrm{C}_{2} \mathrm{H}_{4}\right)_{2}\right]_{2}(5.49 \mathrm{mg}, 0.00967 \mathrm{mmol}), \mathbf{L 3}(23.8 \mathrm{mg}, 0.0202 \mathrm{mmol}), 18$ (51.9 mg, $0.203 \mathrm{mmol})$, tert-butylethylene (35.0 mg, $0.416 \mathrm{mmol})$, and mesitylene $(0.4 \mathrm{~mL}) .19$ (38.7 $\mathrm{mg}, 0.153 \mathrm{mmol}, 75 \%$ ) was obtained as a colorless oil after purification by column chromatography on silica gel (eluent: hexane: $\mathrm{CH}_{2} \mathrm{Cl}_{2}: \mathrm{Et}_{3} \mathrm{~N}=45: 3: 2$ ). 19: ${ }^{1} \mathrm{H} \mathrm{NMR}$ (400 MHz, $\left.\mathrm{CDCl}_{3}\right) \delta 7.28$ [d (AB pattern), $J=8.4 \mathrm{~Hz}, 1 \mathrm{H}$ ] 7.23 [d (AB pattern), $\left.J=8.4 \mathrm{~Hz}, 1 \mathrm{H}\right], 6.71(\mathrm{~d}, J$ $=0.8 \mathrm{~Hz}, 1 \mathrm{H}), 3.96(\mathrm{~s}, 3 \mathrm{H}), 3.25(\mathrm{q}, J=7.6 \mathrm{~Hz}, 2 \mathrm{H}), 2.29(\mathrm{~d}, J=0.8 \mathrm{~Hz}, 3 \mathrm{H}), 1.32(\mathrm{t}, J=7.6 \mathrm{~Hz}$, $3 \mathrm{H}) .{ }^{13} \mathrm{C}$ NMR $\left(101 \mathrm{MHz}, \mathrm{CDCl}_{3}\right) \delta 135.4,129.7,129.5,126.8,123.9,119.0,118.0,110.1,36.8$, 24.7, 15.6, 9.4. HRMS (APCI, positive) $m / z$ calcd for $\mathrm{C}_{12} \mathrm{H}_{15} \mathrm{BrN}^{+}[\mathrm{M}+\mathrm{H}]^{+}: 252.0382$, found: 252.0385 .

\section{Asymmetric Conversion of 20 Affording Enantioenriched Indolines 21 (Table 2)}

\section{0-1. Preparation of 20a-e}

\section{2-Isopropyl- $N, N$-dimethylaniline (20a)}

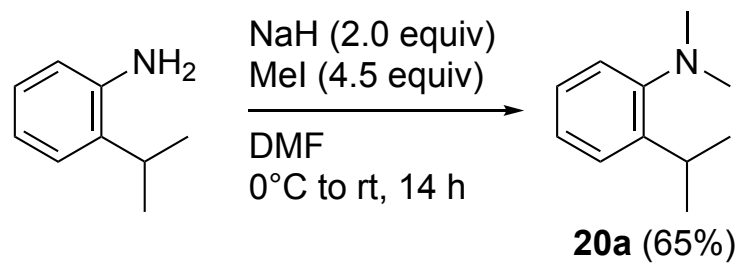

According to the procedure given for 1a, 20a $(1.19 \mathrm{~g}, 7.29 \mathrm{mmol}, 65 \%$ yield) was prepared as a colorless oil from 2-isopropylaniline (TCI, $1.53 \mathrm{~g}, 11.3 \mathrm{mmol}$ ). 20a: ${ }^{1} \mathrm{H}$ NMR (400 MHz, $\left.\mathrm{CDCl}_{3}\right) \delta 7.29(\mathrm{dd}, J=7.6,1.6 \mathrm{~Hz}, 1 \mathrm{H}), 7.07-7.20(\mathrm{~m}, 3 \mathrm{H}), 3.58$ (septet, $\left.J=6.8 \mathrm{~Hz}, 1 \mathrm{H}\right), 2.71(\mathrm{~s}$, $6 \mathrm{H}), 1.25(\mathrm{~d}, J=6.8 \mathrm{~Hz}, 6 \mathrm{H}) .{ }^{13} \mathrm{C} \mathrm{NMR}\left(101 \mathrm{MHz}, \mathrm{CDCl}_{3}\right) \delta 151.9,144.3,126.6,126.3,124.1$, 119.7, 46.0, 26.8, 24.3. HRMS (APCI, positive) $m / z$ calcd for $\mathrm{C}_{11} \mathrm{H}_{18} \mathrm{~N}^{+}[\mathrm{M}+\mathrm{H}]^{+}:$164.1434, found: 164.1432 . 


\section{$N, N$-Dimethyl-2-(1-phenylethyl)aniline (20b)}<smiles>C=C(c1ccccc1)c1ccccc1N(C)C</smiles>

S6
$\mathrm{Pd} / \mathrm{C}(10 \mathrm{~mol} \%$ of $\mathrm{Pd})$ $\mathrm{H}_{2}$ (balloon)

EtOAc

$\mathrm{rt}, 9 \mathrm{~h}$<smiles>CC(c1ccccc1)c1ccccc1N(C)C</smiles>

$20 \mathrm{~b}(89 \%)$

A $100 \mathrm{~mL}$ two-neck flask, equipped with a magnetic stirring bar, a rubber septum, and a three-way stopcock, was charged with Pd/C (Aldrich, 5 wt\% Pd, 3.30 g, 0.75 mmol of Pd). The flask was evacuated and backfilled with nitrogen. $\mathrm{H}_{2}$ balloon was attached, and the flask was evacuated and backfilled with $\mathrm{H}_{2}$. EtOAc $(45 \mathrm{~mL})$ and $\mathbf{S 6}^{7}(1.67 \mathrm{~g}, 7.48 \mathrm{mmol})$ were added to the flask, and the mixture was stirred for $9 \mathrm{~h}$ at room temperature. Insoluble materials were removed by filtration thought a pad of celite. $20 \mathrm{~b}(1.57 \mathrm{~g}, 6.97 \mathrm{mmol}, 89 \%)$ was obtained as a colorless oil after purification by Kugelrhor distillation $\left(110{ }^{\circ} \mathrm{C}, 0.3\right.$ Torr). $20 b^{7}:{ }^{1} \mathrm{H}$ NMR (400 $\left.\mathrm{MHz}, \mathrm{CDCl}_{3}\right) \delta 7.11-7.28(\mathrm{~m}, 8 \mathrm{H}), 7.03-7.08(\mathrm{~m}, 1 \mathrm{H}), 4.86(\mathrm{q}, J=7.2 \mathrm{~Hz}, 1 \mathrm{H}), 2.62(\mathrm{~s}, 6 \mathrm{H}), 1.59$ $(\mathrm{d}, J=7.2 \mathrm{~Hz}, 3 \mathrm{H}) .{ }^{13} \mathrm{C} \mathrm{NMR}\left(101 \mathrm{MHz}, \mathrm{CDCl}_{3}\right) \delta 152.4,147.0,142.1,128.4,128.1,127.6$, $126.7,125.5,124.1,120.4,45.8,37.4,22.3$.

\section{$N, N$-Dimethyl-2-[1-(4-ethylphenyl)ethyl]aniline (20c)}

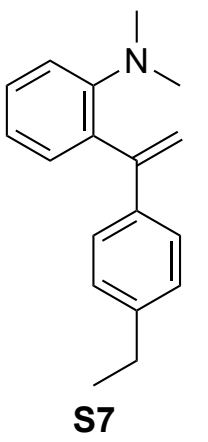

$\mathrm{Pd} / \mathrm{C}(10 \mathrm{~mol} \%$ of $\mathrm{Pd})$ $\mathrm{H}_{2}$ (balloon)

\section{EtOAc}

rt, $17 \mathrm{~h}$

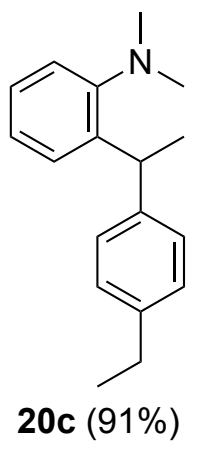

According to the procedure given for 20b, 20c (0.920 g, $3.63 \mathrm{mmol}, 91 \%$ yield) was prepared as a colorless oil from $\mathbf{S 7}{ }^{7}$ (1.00 g, $\left.3.98 \mathrm{mmol}\right) .20 \mathrm{c}:{ }^{1} \mathrm{H} \mathrm{NMR}\left(400 \mathrm{MHz}, \mathrm{CDCl}_{3}\right) \delta 7.13-7.24(\mathrm{~m}$, $5 \mathrm{H}), 7.02-7.12(\mathrm{~m}, 3 \mathrm{H}), 4.84(\mathrm{q}, J=7.2 \mathrm{~Hz}, 1 \mathrm{H}), 2.64(\mathrm{~s}, 6 \mathrm{H}), 2.61$ (q, J=7.6 Hz, 2H), 1.59 (d, $J$ $=7.2 \mathrm{~Hz}, 3 \mathrm{H}), 1.21(\mathrm{t}, J=7.6 \mathrm{~Hz}, 3 \mathrm{H}) .{ }^{13} \mathrm{C} \mathrm{NMR}\left(101 \mathrm{MHz}, \mathrm{CDCl}_{3}\right) \delta 152.5,144.3,142.5,141.5$, 
128.6, 127.73, 127.66, 126.7, 124.2, 120.5, 46.0, 37.1, 28.5, 22.6, 15.7. HRMS (ESI) $\mathrm{m} / z$ calcd for $\mathrm{C}_{18} \mathrm{H}_{24} \mathrm{~N}^{+}[\mathrm{M}+\mathrm{H}]^{+}:$254.1903, found: 254.1905 .

\section{$N, N$-Dimethyl-2-[1-(4-methoxyphenyl)ethyl]aniline (20d)}<smiles>C=C(c1ccc(OC)cc1)c1ccccc1N(C)C</smiles>

S8
$\mathrm{Pd} / \mathrm{C}(10 \mathrm{~mol} \%$ of $\mathrm{Pd})$ $\mathrm{H}_{2}$ (balloon)

EtOAc

rt, $17 \mathrm{~h}$<smiles>COc1ccc(C(C)c2ccccc2N(C)C)cc1</smiles>

20d (92\%)

According to the procedure given for 20b, 20d (1.17 g, $4.58 \mathrm{mmol}, 92 \%$ yield) was prepared as a colorless oil from $\mathbf{S 8}^{7}$ (1.26 g, $\left.4.97 \mathrm{mmol}\right) .20 \mathrm{~d}:{ }^{1} \mathrm{H}$ NMR (400 MHz, $\left.\mathrm{CDCl}_{3}\right) \delta$ 7.15-7.22 (m, 5H), 7.03-7.10 (m, 1H), 6.79-6.84 (m, 2H), 4.82 (q, J= 7.2 Hz, 1H), 3.78 (s, 3H), $2.64(\mathrm{~s}, 6 \mathrm{H})$, $1.57(\mathrm{~d}, J=7.2 \mathrm{~Hz}, 3 \mathrm{H}) .{ }^{13} \mathrm{C} \mathrm{NMR}\left(101 \mathrm{MHz}, \mathrm{CDCl}_{3}\right) \delta 157.6,152.5,142.5,139.3,128.6,128.5$, 126.7, 124.2, 120.5, 113.6, 55.3, 46.0, 36.7, 22.7. HRMS (ESI) $m / z$ calcd for $\mathrm{C}_{17} \mathrm{H}_{22} \mathrm{NO}^{+}[\mathrm{M}+$ $\mathrm{H}]^{+}: 256.1696$, found: 256.1697 .

\section{$N, N$-Dimethyl-2-[1-(2-naphthyl)ethyl]aniline (20e)}<smiles>C=C(c1ccc2ccccc2c1)c1ccccc1N(C)C</smiles>

S9
$\mathrm{Pd} / \mathrm{C}(10 \mathrm{~mol} \%$ of $\mathrm{Pd})$ $\mathrm{H}_{2}$ (balloon)

EtOAc rt, $2 \mathrm{~h}$<smiles>CC(c1ccc2ccccc2c1)c1ccccc1N(C)C</smiles>

20e (65\%)

According to the procedure given for $20 \mathrm{~b}, \mathbf{2 0 e}(0.417 \mathrm{~g}, 1.11 \mathrm{mmol}, 65 \%$ yield $)$ was prepared as a colorless oil from $\mathbf{S 9}{ }^{7}$ (0.465 g, $\left.1.70 \mathrm{mmol}\right)$. 20e: ${ }^{1} \mathrm{H}$ NMR $\left(400 \mathrm{MHz}, \mathrm{CDCl}_{3}\right) \delta 7.78-7.84$ (m, 2H), 7.72-7.77 (m, 2H), 7.37-7.50 (m, 3H), 7.21-7.28 (m, 3H), 7.06-7.13 (m, 1H), $5.06(\mathrm{q}, J$

$=7.2 \mathrm{~Hz}, 1 \mathrm{H}), 2.69(\mathrm{~s}, 6 \mathrm{H}), 1.73(\mathrm{~d}, J=7.2 \mathrm{~Hz}, 3 \mathrm{H}) .{ }^{13} \mathrm{C} \mathrm{NMR}\left(101 \mathrm{MHz}, \mathrm{CDCl}_{3}\right) \delta 152.6,144.7$, 
$142.0,133.6,132.0,128.8,127.9,127.8,127.6,127.5,126.9,125.9,125.3,125.0,124.3,120.6$, 46.0, 37.7, 22.3. HRMS (ESI) $m / z$ calcd for $\mathrm{C}_{20} \mathrm{H}_{22} \mathrm{~N}^{+}[\mathrm{M}+\mathrm{H}]^{+}: 276.1747$, found: 276.1750 .

\section{$N, N$-Dimethyl-2-(1-phenylpropan-2-yl)aniline (20f)}<smiles>C=C(Cc1ccccc1)c1ccccc1N(C)C</smiles>

S10
$\mathrm{Pd} / \mathrm{C}(20 \mathrm{~mol} \%$ of $\mathrm{Pd})$ $\mathrm{H}_{2}$ (balloon)

EtOAc

$\mathrm{rt}, 7 \mathrm{~h}$<smiles>CC(Cc1ccccc1)c1ccccc1N(C)C</smiles>

$20 f(87 \%)$

According to the procedure given for $\mathbf{2 0 b}, \mathbf{2 0 f}$ (1.98 g, $8.27 \mathrm{mmol}, 87 \%$ yield) was prepared as a colorless oil from $\mathbf{S 1 0}^{7}$ (2.25 g, $\left.9.48 \mathrm{mmol}\right) .20 f:{ }^{1} \mathrm{H}$ NMR (400 MHz, $\left.\mathrm{CDCl}_{3}\right) \delta$ 7.30-7.48 (m, 1H), 7.20-7.62 (m, 2H), 6.98-7.20 (m, 6H), 3.69-3.80 (m, 1H), 2.92 [dd (AB pattern), $J=13.6$, $6.4 \mathrm{~Hz}, 1 \mathrm{H}$ ], 2.72 [dd (AB pattern), $J=13.6,8.8 \mathrm{~Hz}, 1 \mathrm{H}$ ], 2.60 (s, 6H), 1.19 (d, $J=6.8 \mathrm{~Hz}, 3 \mathrm{H})$.

${ }^{13} \mathrm{C}$ NMR $\left(101 \mathrm{MHz}, \mathrm{CDCl}_{3}\right) \delta 152.5,142.9,141.5,129.3,128.1,126.9,126.5,125.8,124.1$, 120.2, 45.9, 45.0, 34.0, 21.2. HRMS (ESI) $m / z$ calcd for $\mathrm{C}_{17} \mathrm{H}_{22} \mathrm{~N}^{+}[\mathrm{M}+\mathrm{H}]^{+}: 240.1747$, found: 240.1747 .

\section{0-2. Reaction of 20a-e (Table 2)}

The Reaction of 20a to Afford 1,3,3-Trimethylindoline (21a) (entry 1)<smiles>CC(C)c1ccccc1N(C)C</smiles>

20a

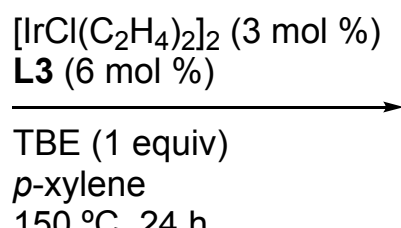

$150^{\circ} \mathrm{C}, 24 \mathrm{~h}$

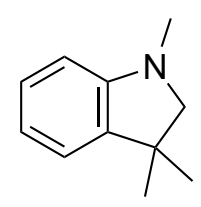

21a $(77 \%)$

According to the general procedure $A$ described in section 5, the reaction was carried out at $150{ }^{\circ} \mathrm{C}$ for $24 \mathrm{~h}$ using $\left[\operatorname{IrCl}\left(\mathrm{C}_{2} \mathrm{H}_{4}\right)_{2}\right]_{2}(3.55 \mathrm{mg}, 0.00625 \mathrm{mmol}), \mathbf{L 3}(14.2 \mathrm{mg}, 0.0120 \mathrm{mmol}), \mathbf{2 0 a}$ (34.0 mg, $0.208 \mathrm{mmol})$, tert-butylethylene $(17.6 \mathrm{mg}, 0.209 \mathrm{mmol})$, and $p$-xylene $(0.4 \mathrm{~mL})$. Since mesitylene was difficult to separate from 21a, $p$-xylene was used as a solvent. 21a (26.0 mg, $0.161 \mathrm{mmol}, 77 \%$ ) was obtained as a colorless oil after purification by column chromatography on silica gel (eluent: hexane: $\left.\mathrm{Et}_{2} \mathrm{O}: \mathrm{Et}_{3} \mathrm{~N}=45: 1: 1\right) .21 \mathrm{a}:{ }^{1} \mathrm{H} \mathrm{NMR}\left(400 \mathrm{MHz}, \mathrm{CDCl}_{3}\right) \delta 7.12(\mathrm{t}, J=$ $7.6 \mathrm{~Hz}, 1 \mathrm{H}), 7.03$ (d, $J=7.2 \mathrm{~Hz}, 1 \mathrm{H}), 6.73$ (t, $J=7.6 \mathrm{~Hz}, 1 \mathrm{H}), 6.51$ (d, $J=7.6 \mathrm{~Hz}, 1 \mathrm{H}), 3.09$ (s, 
2H), $2.77(\mathrm{~s}, 3 \mathrm{H}), 1.32(\mathrm{~s}, 6 \mathrm{H}) .{ }^{13} \mathrm{C} \mathrm{NMR}\left(101 \mathrm{MHz}, \mathrm{CDCl}_{3}\right) \delta 152.1,139.4,127.6,121.7,118.0$, 107.5, 70.4, 40.4, 36.2, 27.5. HRMS (APCI, positive) $m / z$ calcd for $\mathrm{C}_{11} \mathrm{H}_{16} \mathrm{~N}^{+}[\mathrm{M}+\mathrm{H}]^{+}: 162.1277$, found: 162.1276 .

The Reaction of 20b to Afford 1,3-Dimethyl-3-phenylindoline (21b) (entry 2)<smiles>CC(c1ccccc1)c1ccccc1N(C)C</smiles>

$20 \mathrm{~b}$
$\left[\mathrm{IrCl}\left(\mathrm{C}_{2} \mathrm{H}_{4}\right)_{2}\right]_{2}(3 \mathrm{~mol} \%)$

(S)-L3 (6 mol \%)

TBE (1 equiv)

mesitylene

$150^{\circ} \mathrm{C}, 24 \mathrm{~h}$

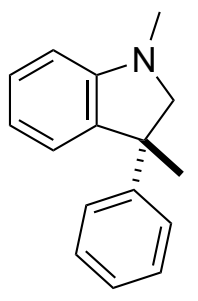

$(R)-21 b(91 \%, 91 \%$ ee $)$

According to the general procedure $A$ described in section 5, the reaction was carried out at $150{ }^{\circ} \mathrm{C}$ for $24 \mathrm{~h}$ using $\left[\operatorname{IrCl}\left(\mathrm{C}_{2} \mathrm{H}_{4}\right)_{2}\right]_{2}(3.51 \mathrm{mg}, 0.00618 \mathrm{mmol}),(S)-\mathbf{L 3}(15.1 \mathrm{mg}, 0.0128 \mathrm{mmol})$, 20c (46.1 mg, $0.205 \mathrm{mmol})$, tert-butylethylene (18.7 mg, $0.222 \mathrm{mmol})$, and mesitylene $(0.4 \mathrm{~mL})$. 21c (41.8 mg, $0.187 \mathrm{mmol}, 91 \%)$ was obtained as a colorless oil after purification by column chromatography on silica gel (eluent: hexane: $\mathrm{Et}_{2} \mathrm{O}=10: 1$ ). Enantiomeric excess of 21c was determined to be $91 \%$ by SFC analysis [column: Daicel Chiralcel OD-H (4.6 mm x $250 \mathrm{~mm})$; eluent: $\mathrm{CO}_{2}: 2-\mathrm{PrOH}=100: 2$; flow rate: $3.06 \mathrm{~mL} / \mathrm{min}$; detection wavelength: $220 \mathrm{~nm} ; \mathrm{T}_{\mathrm{R}}=6.7$ (major), 7.5 (minor) min]. The absolute configuration of the major enantiomer was assigned as $R$ on the basis of the previous study. ${ }^{7}(R)-\mathbf{2 1 b ^ { 7 }}{ }^{7}{ }^{1} \mathrm{H}$ NMR $\left(400 \mathrm{MHz}, \mathrm{CDCl}_{3}\right) \delta 7.25-7.34(\mathrm{~m}, 4 \mathrm{H})$, 7.16-7.24 (m, 1H), $7.15(\mathrm{dd}, J=7.6,1.6 \mathrm{~Hz}, 1 \mathrm{H}), 6.96(\mathrm{dd}, J=7.2,1.6 \mathrm{~Hz}, 1 \mathrm{H}), 6.74(\mathrm{td}, J=7.6$, $0.8 \mathrm{~Hz}, 1 \mathrm{H}), 6.58(\mathrm{~d}, J=8.0 \mathrm{~Hz}, 1 \mathrm{H}), 3.53$ (d, $J=8.8 \mathrm{~Hz}, 1 \mathrm{H}), 3.31$ (d, $J=8.8 \mathrm{~Hz}, 1 \mathrm{H}), 2.78$ (s, $3 \mathrm{H}), 1.71(\mathrm{~s}, 3 \mathrm{H}) .{ }^{13} \mathrm{C} \mathrm{NMR}\left(101 \mathrm{MHz}, \mathrm{CDCl}_{3}\right) \delta 152.7,147.7,137.8,128.3,128.0,126.7,126.3$, 123.9, 118.3, 107.8, 72.3, 48.4, 36.1, 26.4. $[\alpha]^{26}+31.2$ (c $0.52, \mathrm{CHCl}_{3}, 91 \%$ ee). 


\section{The Reaction of 20c to Afford 3-(4-Ethylphenyl)-1,3-dimethylindoline (21c) (entry 3)}

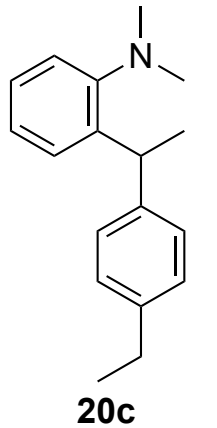

$\left[\mathrm{IrCl}\left(\mathrm{C}_{2} \mathrm{H}_{4}\right)_{2}\right]_{2}(3 \mathrm{~mol} \%)$ (S)-L3 $(6 \mathrm{~mol} \%)$

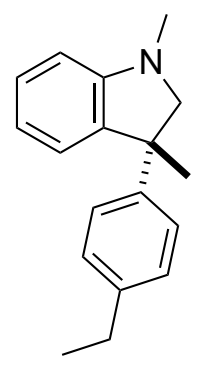

TBE (1 equiv) mesitylene $150^{\circ} \mathrm{C}, 24 \mathrm{~h}$

$(R)-21 \mathrm{c}(81 \%, 92 \%$ ee $)$

According to the general procedure $A$ described in section 5, the reaction was carried out at $150{ }^{\circ} \mathrm{C}$ for $24 \mathrm{~h}$ using $\left[\operatorname{IrCl}\left(\mathrm{C}_{2} \mathrm{H}_{4}\right)_{2}\right]_{2}(3.74 \mathrm{mg}, 0.00659 \mathrm{mmol}),(S)-\mathbf{L 3}(14.4 \mathrm{mg}, 0.0122 \mathrm{mmol})$, 20c (52.7 mg, $0.208 \mathrm{mmol})$, tert-butylethylene $(20.8 \mathrm{mg}, 0.247 \mathrm{mmol})$, and mesitylene $(0.4 \mathrm{~mL})$. 21c (42.6 mg, $0.169 \mathrm{mmol}, 81 \%$ ) was obtained as a colorless oil after purification by column chromatography on silica gel (eluent: hexane: $\mathrm{Et}_{2} \mathrm{O}=10: 1$ ). Enantiomeric excess of 21c was determined to be $92 \%$ by SFC analysis [column: Daicel Chiralcel OD-H (4.6 mm x $250 \mathrm{~mm})$; eluent: $\mathrm{CO}_{2}: 2-\mathrm{PrOH}=100: 3$; flow rate: $3.09 \mathrm{~mL} / \mathrm{min}$; detection wavelength: $220 \mathrm{~nm} ; \mathrm{T}_{\mathrm{R}}=5.5$ (major), 6.3 (minor) min]. The absolute configuration of the major enantiomer was assigned as $R$ on the basis of the previous study. ${ }^{7}(R)-21 \mathrm{c}^{7}:{ }^{1} \mathrm{H}$ NMR (400 MHz, $\left.\mathrm{CDCl}_{3}\right) \delta 7.22-7.26(\mathrm{~m}, 2 \mathrm{H})$, $7.16(\mathrm{td}, J=7.6,1.2 \mathrm{~Hz}, 1 \mathrm{H}), 7.10-7.14(\mathrm{~m}, 2 \mathrm{H}), 6.98(\mathrm{dd}, J=7.2,0.8 \mathrm{~Hz}, 1 \mathrm{H}), 6.75(\mathrm{td}, J=7.2$, $0.8 \mathrm{~Hz}, 1 \mathrm{H}), 6.58(\mathrm{~d}, J=8.0 \mathrm{~Hz}, 1 \mathrm{H}), 3.52(\mathrm{~d}, J=8.8 \mathrm{~Hz}, 1 \mathrm{H}), 3.30(\mathrm{~d}, J=8.8 \mathrm{~Hz}, 1 \mathrm{H}), 2.78(\mathrm{~s}$, $3 \mathrm{H}), 2.62(\mathrm{q}, J=7.6 \mathrm{~Hz}, 2 \mathrm{H}), 1.71(\mathrm{~s}, 3 \mathrm{H}), 1.22(\mathrm{t}, J=7.6 \mathrm{~Hz}, 3 \mathrm{H}) .{ }^{13} \mathrm{C} \mathrm{NMR}\left(101 \mathrm{MHz}, \mathrm{CDCl}_{3}\right)$ $\delta 152.7,144.9,142.1,137.9,127.9,127.8,126.7,123.8,118.2,107.8,72.3,48.1,36.2,28.4,26.5$, 15.6. $[\alpha]_{\mathrm{D}}^{28}+27.7$ (c $0.49, \mathrm{CHCl}_{3}, 92 \%$ ee).

The Reaction of 20d to Afford 3-(4-Methoxyphenyl)-1,3-dimethylindoline (21d) (entry 4)<smiles>COc1ccc(C(C)c2ccccc2N(C)C)cc1</smiles>

20d
$\left[\mathrm{IrCl}\left(\mathrm{C}_{2} \mathrm{H}_{4}\right)_{2}\right]_{2}(3 \mathrm{~mol} \%)$ (S)-L5 (6 mol \%)

TBE (1 equiv) mesitylene $150^{\circ} \mathrm{C}, 24 \mathrm{~h}$<smiles>COc1ccc([C@@]2(C)CN(C)c3ccccc32)cc1</smiles>

(R)-21d (86\%, 94\% ee) 
According to the general procedure $A$ described in section 5, the reaction was carried out at $150{ }^{\circ} \mathrm{C}$ for $24 \mathrm{~h}$ using $\left[\operatorname{IrCl}\left(\mathrm{C}_{2} \mathrm{H}_{4}\right)_{2}\right]_{2}(3.66 \mathrm{mg}, 0.00645 \mathrm{mmol}),(S)-\mathbf{L 5}(15.1 \mathrm{mg}, 0.0131 \mathrm{mmol})$, 20d (53.5 mg, $0.210 \mathrm{mmol})$, tert-butylethylene (19.4 mg, $0.231 \mathrm{mmol})$, and mesitylene $(0.4 \mathrm{~mL})$. 21d (45.7 mg, $0.180 \mathrm{mmol}, 86 \%$ ) was obtained as a colorless oil after purification by column chromatography on silica gel (eluent: hexane: $\mathrm{Et}_{2} \mathrm{O}=5: 1$ ). Enantiomeric excess of 21d was determined to be $94 \%$ by SFC analysis [column: Daicel Chiralcel OD-H (4.6 mm x $250 \mathrm{~mm})$; eluent: $\mathrm{CO}_{2}: 2-\mathrm{PrOH}=100: 5$; flow rate: $3.15 \mathrm{~mL} / \mathrm{min}$; detection wavelength: $220 \mathrm{~nm} ; \mathrm{T}_{\mathrm{R}}=6.1$ (major), 11.1 (minor) min]. The absolute configuration of the major enantiomer was assigned as $R$ on the basis of the previous study. ${ }^{7}$ The reaction using $(S)$ - $\mathbf{L 3}$ afforded also $R$ isomer as the major enantiomer (68\% yield, 89\% ee). (R)-21d ${ }^{7}:{ }^{1} \mathrm{H}$ NMR (400 MHz, $\left.\mathrm{CDCl}_{3}\right) \delta$ 7.21-7.25 (m, 2H), $7.15(\mathrm{td}, J=7.6,1.2 \mathrm{~Hz}, 1 \mathrm{H}), 6.93-6.97$ (m, 1H), 6.80-6.84 (m, 2H), 6.73 (td, $J=7.6,0.8 \mathrm{~Hz}$, $1 \mathrm{H}), 6.57(\mathrm{~d}, J=8.0 \mathrm{~Hz}, 1 \mathrm{H}), 3.78(\mathrm{~s}, 3 \mathrm{H}), 3.48(\mathrm{~d}, J=8.8 \mathrm{~Hz}, 1 \mathrm{H}), 3.28(\mathrm{~d}, J=8.8 \mathrm{~Hz}, 1 \mathrm{H})$, $2.77(\mathrm{~s}, 3 \mathrm{H}), 1.69(\mathrm{~s}, 3 \mathrm{H}) .{ }^{13} \mathrm{C} \mathrm{NMR}\left(101 \mathrm{MHz}, \mathrm{CDCl}_{3}\right) \delta 158.0,152.6,139.8,138.0,127.9$, $127.8,123.7,118.2,113.6,107.8,72.4,55.3,47.8,36.2,26.5 .[\alpha]^{26}{ }_{\mathrm{D}}+25.2\left(\mathrm{c} 0.51, \mathrm{CHCl}_{3}, 94 \%\right.$ ee).

The Reaction of 20e to Afford 1,3-Dimethyl-3-(naphthalen-2-yl)indoline (21e) (entry 5)

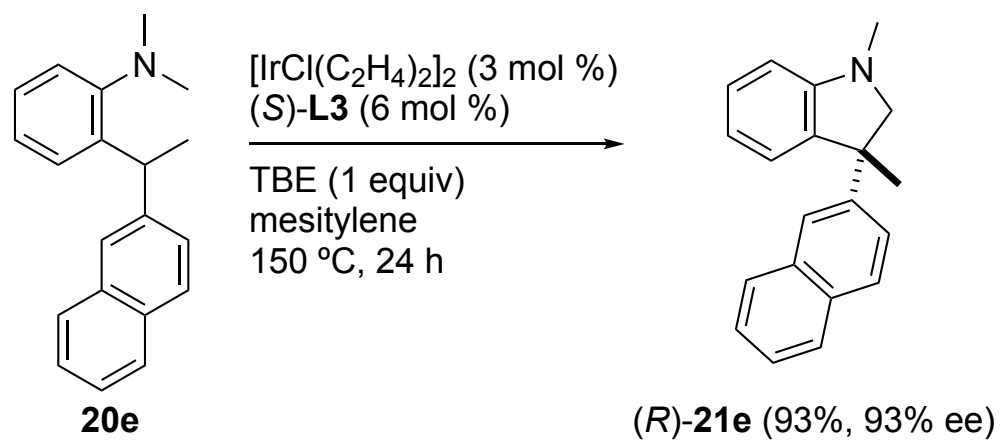

According to the general procedure $A$ described in section 5, the reaction was carried out at $150{ }^{\circ} \mathrm{C}$ for $24 \mathrm{~h}$ using $\left[\operatorname{IrCl}\left(\mathrm{C}_{2} \mathrm{H}_{4}\right)_{2}\right]_{2}(3.46 \mathrm{mg}, 0.00610 \mathrm{mmol}),(S)-\mathbf{L 3}(14.6 \mathrm{mg}, 0.0124 \mathrm{mmol})$, 20e (55.6 mg, $0.202 \mathrm{mmol})$, tert-butylethylene $(18.1 \mathrm{mg}, 0.215 \mathrm{mmol})$, and mesitylene $(0.4 \mathrm{~mL})$. 21e (51.3 mg, $0.188 \mathrm{mmol}, 93 \%)$ was obtained as a colorless oil after purification by column chromatography on silica gel (eluent: hexane: $\mathrm{Et}_{2} \mathrm{O}=10: 1$ ). Enantiomeric excess of 21e was determined to be $93 \%$ by SFC analysis [column: Daicel Chiralcel OD-H (4.6 mm x $250 \mathrm{~mm})$; 
eluent: $\mathrm{CO}_{2}: 2-\mathrm{PrOH}=100: 5$; flow rate: $3.15 \mathrm{~mL} / \mathrm{min}$; detection wavelength: $220 \mathrm{~nm} ; \mathrm{T}_{\mathrm{R}}=8.8$ (major), 10.6 (minor) min]. The absolute configuration of the major enantiomer was assigned as $R$ on the basis of the previous study. ${ }^{7}(R)-21 e^{7}:{ }^{1} \mathrm{H}$ NMR $\left(400 \mathrm{MHz}, \mathrm{CDCl}_{3}\right) \delta 7.72-7.80(\mathrm{~m}, 4 \mathrm{H})$, $7.48(\mathrm{dd}, J=8.8,1.6 \mathrm{~Hz}, 1 \mathrm{H}), 7.40-7.46(\mathrm{~m}, 2 \mathrm{H}), 7.19$ (t, $J=7.6 \mathrm{~Hz}, 1 \mathrm{H}), 6.98(\mathrm{~d}, J=7.2 \mathrm{~Hz}$, 1H), 6.75 (t, $J=7.2 \mathrm{~Hz}, 1 \mathrm{H}), 6.61(\mathrm{~d}, J=7.6 \mathrm{~Hz}, 1 \mathrm{H}), 3.63(\mathrm{~d}, J=8.8 \mathrm{~Hz}, 1 \mathrm{H}), 3.38(\mathrm{~d}, J=8.8$ $\mathrm{Hz}, 1 \mathrm{H}), 2.81$ (s, 3H), $1.82(\mathrm{~s}, 3 \mathrm{H}) .{ }^{13} \mathrm{C} \mathrm{NMR}\left(101 \mathrm{MHz}, \mathrm{CDCl}_{3}\right) \delta 152.7,144.9,137.7,133.2$, $132.2,128.2,128.1,128.0,127.5,126.0,125.9,125.7,124.7,123.9,118.3,107.9,72.0,48.6$, 36.1, 26.4. $[\alpha]^{27}-30.2$ (c $0.49, \mathrm{CHCl}_{3}, 93 \%$ ee).

\section{The Reaction of 20 fo Afford 3-Benzyl-1,3-dimethylindoline (21f) (entry 6)}

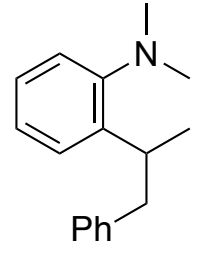

$20 f$
$\left[\mathrm{IrCl}\left(\mathrm{C}_{2} \mathrm{H}_{4}\right)_{2}\right]_{2}(3 \mathrm{~mol} \%)$ (S)-L5 (6 mol \%)

TBE (1 equiv) mesitylene $150{ }^{\circ} \mathrm{C}, 24 \mathrm{~h}$

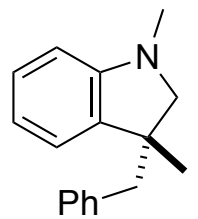

(R)-21f (87\%, 77\% ee)

According to the general procedure $A$ described in section 5, the reaction was carried out at $150{ }^{\circ} \mathrm{C}$ for $24 \mathrm{~h}$ using $\left[\operatorname{IrCl}\left(\mathrm{C}_{2} \mathrm{H}_{4}\right)_{2}\right]_{2}(3.53 \mathrm{mg}, 0.00622 \mathrm{mmol}),(S)-\mathbf{L 5}(14.8 \mathrm{mg}, 0.0129 \mathrm{mmol})$, $20 f(49.2 \mathrm{mg}, 0.206 \mathrm{mmol})$, tert-butylethylene (19.1 mg, $0.227 \mathrm{mmol})$, and mesitylene $(0.4 \mathrm{~mL})$. $21 \mathrm{f}(42.6 \mathrm{mg}, 0.180 \mathrm{mmol}, 87 \%$ ) was obtained as a colorless oil after purification by column chromatography on silica gel (eluent: hexane: $\mathrm{Et}_{2} \mathrm{O}=10: 1$ ). Enantiomeric excess of $21 f$ was determined to be $77 \%$ by SFC analysis [column: Daicel Chiralcel OJ-H (4.6 mm x $250 \mathrm{~mm})$; eluent: $\mathrm{CO}_{2}: 2-\mathrm{PrOH}=100: 3$; flow rate: $3.09 \mathrm{~mL} / \mathrm{min}$; detection wavelength: $220 \mathrm{~nm} ; \mathrm{T}_{\mathrm{R}}=3.5$ (minor), 4.8 (major) min]. The absolute configuration of the major enantiomer was assigned as $R$ on the basis of the previous study. ${ }^{7}$ The reaction using $(S)$ - $\mathbf{L 3}$ afforded also $R$ isomer as the major enantiomer (74\% yield, 74\% ee). (R)-21f ${ }^{7}:{ }^{1} \mathrm{H}$ NMR (400 MHz, $\left.\mathrm{CDCl}_{3}\right) \delta$ 7.19-7.29 (m, 3H), $7.12(\mathrm{td}, J=8.0,1.2 \mathrm{~Hz}, 1 \mathrm{H}), 7.05-7.09(\mathrm{~m}, 2 \mathrm{H}), 6.82(\mathrm{dd}, J=7.6,1.2 \mathrm{~Hz}, 1 \mathrm{H}), 6.68(\mathrm{td}, J=7.6$, $0.8 \mathrm{~Hz}, 1 \mathrm{H}), 6.51(\mathrm{~d}, J=7.6 \mathrm{~Hz}, 1 \mathrm{H}), 3.34(\mathrm{~d}, J=8.8 \mathrm{~Hz}, 1 \mathrm{H}), 2.89$ [d (AB pattern), $J=13.2 \mathrm{~Hz}$, $1 \mathrm{H}$ ], $2.842(\mathrm{~d}, J=8.8 \mathrm{~Hz}, 1 \mathrm{H}), 2.841$ [d (AB pattern), $J=13.2 \mathrm{~Hz}, 1 \mathrm{H}$ ], 2.75 (s, 3H), 1.28 (s, 3H).

${ }^{13} \mathrm{C}$ NMR $\left(101 \mathrm{MHz}, \mathrm{CDCl}_{3}\right) \delta 152.4,138.6,137.8,130.7,127.9,127.8,126.3,122.8,117.7$, 107.5, 68.1, 45.9, 44.7, 36.1, 24.3. $[\alpha]_{\mathrm{D}}^{25}-70.8$ (c $0.52, \mathrm{CHCl}_{3}, 77 \%$ ee). 
We previously revealed in the $\operatorname{Ir} /(S)$-L3-catalyzed intramolecular $\mathrm{C}\left(\mathrm{sp}^{3}\right)-\mathrm{H}$ addition reaction, $N, N$-dimethyl-2-(3-phenylprop-1-en-2-yl)aniline (22) afforded $(R)$-21f, whereas $(S)$-21f was formed from $N, N$-dimethyl-2-(1-phenylprop-1-en-2-yl)aniline (23) (Scheme S4). ${ }^{7}$ In the reaction of 20f with $\operatorname{Ir} /(S)$-L3 and $\operatorname{Ir} /(S)$-L5, $(R)$-21f was formed as a major enantiomer (74 and 77\% ees, respectively). The absolute configuration of the product $21 \mathbf{f}$ suggests that the $\mathrm{C}-\mathrm{H}$ addition took place through terminal alkene $\mathbf{2 2}$ generated by dehydrogenation rather than the stilbene-type internal alkene $\mathbf{2 3}$.

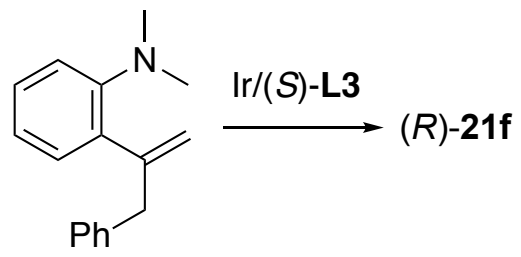

22

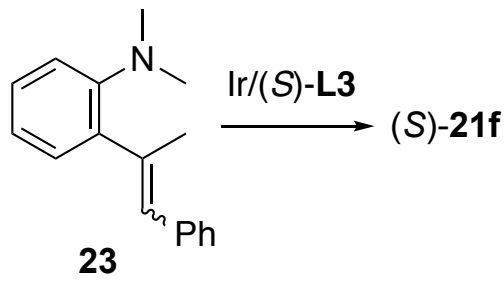

Scheme S4. Previous Findings in Enantioselective Cyclization of 22 and 23

\section{References}

(1) Arthurs, M. A.; Bickerton, J.; Stobart, S. R.; Wang, J. Pyrazolyl-Bridged Iridium Dimers. 17. Tetrakis(alkene)diiridium(I) Complexes: $\left[\operatorname{Ir}\left(\eta^{2}-\mathrm{C}_{2} \mathrm{H}_{4}\right)_{2}(\mu-\mathrm{Cl})\right]_{2}$ as a Precursor to $\left[\operatorname{Ir}\left(\eta^{2}-\mathrm{C}_{2} \mathrm{H}_{4}\right)_{2}(\mu-\mathrm{pz})\right]_{2}$. Stereochemically Nonrigid Behavior of the Analogue $\left[\operatorname{Ir}\left(\eta^{2}-\mathrm{C}_{2} \mathrm{H}_{4}\right)\left(\eta^{2}-\mathrm{C}_{2} \mathrm{~F}_{4}\right)(\mu-\mathrm{Cl})\right]_{2}$, Organometallics 1998, 17, 2743-2750.

(2) Yi, Y.-Q.-Q.; Yang, W.-C.; Zhai, D.-D.; Zhang, X.-Y.; Li, S.-Q.; Guan, B.-T. Nickel-catalyzed $\mathrm{C}-\mathrm{N}$ bond reduction of aromatic and benzylic quaternary ammonium triflates, Chem. Commun. 2016, 52, 10894-10897.

(3) Song, S.; Sun, X.; Li, X.; Yuan, Y.; Jiao, N. Efficient and Practical Oxidative Bromination and Iodination of Arenes and Heteroarenes with DMSO and Hydrogen Halide: A Mild Protocol for Late-Stage Functionalization , Org. Lett. 2015, 17, 2886-2889. 
(4) Altman, R. A.; Shafir, A.; Choi, A.; Lichtor, P. A.; Buchwald, S. L. An Improved Cu-Based Catalyst System for the Reactions of Alcohols with Aryl Halides, J. Org. Chem. 2008, 73, 284-286.

(5) Hartwig, J. F.; Kawatsura, M.; Hauck, S. I.; Shaughnessy, K. H.; Alcazar-Roman, L. M. Room-Temperature Palladium-Catalyzed Amination of Aryl Bromides and Chlorides and Extended Scope of Aromatic C-N Bond Formation with a Commercial Ligand, J. Org. Chem. 1999, 64, 5575-5580.

(6) Gale, D. J.; Wilshire, J. F. K. The amidomethylation of some $N, N$-dialkylanilines (Tscherniac-Einhorn reaction), Aust. J. Chem. 1975, 28, 2447-2458.

(7) Torigoe, T.; Ohmura, T.; Suginome, M. Asymmetric Cycloisomerization of $o$-Alkenyl- $N$-methylanilines to Indolines through Iridium-Catalyzed $\mathrm{C}\left(\mathrm{sp}^{3}\right)-\mathrm{H}$ Addition to Carbon-Carbon Double Bonds, Angew. Chem. Int. Ed. 2017, 56, 14272-14276.

(8) For transfer hydrogenation of C-C unsaturated bonds by Ir/DTBM-SEGPHOS catalyst with THF as a hydrogen donor, see: (a) Torigoe, T.; Ohmura, T.; Suginome, M. Iridium-Catalyzed Intramolecular Methoxy C-H Addition to Carbon-Carbon Triple Bonds: Direct Synthesis of 3-Substituted Benzofurans from o-Methoxyphenylalkynes, Chem. Eur. J. 2016, 22, 1041510419. (b) Ref. 7. (c) Ohmura, T.; Kusaka, S.; Torigoe, T.; Suginome, M. Iridium-Catalyzed $\mathrm{C}\left(\mathrm{sp}^{3}\right)-\mathrm{H}$ Addition of Methyl Ethers across Intramolecular Carbon-Carbon Double Bonds Giving 2,3-Dihydrobenzofurans, Adv. Synth. Catal. 2019, 361, 4448-4453.

(9) Muchalski, H.; Levonyak, A. L.; Xu, L.; Ingold, K. U.; Porter, N. A. Competition H(D) Kinetic Isotope Effects in the Autoxidation of Hydrocarbons, J. Am. Chem. Soc. 2015, 137, 94-97.

(10) Zhan, M.; Zhang, T.; Huang, H.; Xie, Y.; Chen, Y. A simple method for $\alpha$-position deuterated carbonyl compounds with pyrrolidine as catalyst, J. Label. Compd. Radiopharm. 2014, 57, 533-539.

(11) Yamamoto, C.; Takamatsu, K.; Hirano, K.; Miura, M. Copper-Catalyzed Intramolecular Benzylic C-H Amination for the Synthesis of Isoindolinones, J. Org. Chem. 2016, 81, 76757684.

(12) Furukawa, T.; Tobisu, M.; Chatani, N. Nickel-catalyzed borylation of arenes and indoles via C-H bond cleavage, Chem. Commun. 2015, 51, 6508-6511. 
(13) Ishiyama, T.; Takagi, J.; Hartwig, J. F.; Miyaura, N. A Stoichiometric Aromatic C-H Borylation Catalyzed by Iridium(I)/2,2'-Bipyridine Complexes at Room Temperature, Angew. Chem. Int. Ed. 2002, 41, 3056-3058.

(14) Tang, S.; Li, J.-H.; Xie, Y.-X.; Wang, N.-X. Halogenation of Indoles with Copper(II) Halides: Selective Synthesis of 2-Halo-, 3-Halo-, and 2,3-Dibromoindoles, Synthesis 2007, $1535-1541$.

(15) Rosevear, J.; Wilshire, J. F. K. The bromination of some $N, N$-dimethylanilines with dibromoisocyanuric acid, Aust. J. Chem. 1977, 30, 1561-1572.

\section{2. ${ }^{1} \mathrm{H}$ and ${ }^{13} \mathrm{C}$ NMR Spectra of New Compounds and Key Compounds}

${ }^{1} \mathrm{H}$ and ${ }^{13} \mathrm{C}$ NMR spectra of $\mathbf{1 a}-\mathbf{e}, \mathbf{1 r}, \mathbf{1}-\mathbf{h}, \mathbf{S 1}, \mathbf{S 2}, \mathbf{1 i}-\mathbf{j}, \mathbf{S 3}, \mathbf{S 4}, \mathbf{1 k}-\mathbf{q}, \mathbf{4}, \mathbf{6}, \mathbf{8}, \mathbf{9}, \mathbf{2 a}, \mathbf{5}, \mathbf{7}$, 2b-q, 10, S5, 1a-d 2, S5- $_{3}, 1 \mathrm{a}-d_{3}, 1 \mathrm{a}-d_{6}, 2 \mathrm{a}, 13-16,18,19,20 \mathrm{a}, \mathbf{2 0 c}-\mathbf{f}$, and $21 \mathrm{a}$ are given in following pages. ${ }^{1} \mathrm{H}$ and ${ }^{13} \mathrm{C}$ NMR spectra for the reaction of $\mathbf{1} \mathbf{a}-\boldsymbol{d}_{2}$ and $\mathbf{1 a - d _ { 3 }}$ are also provided. 


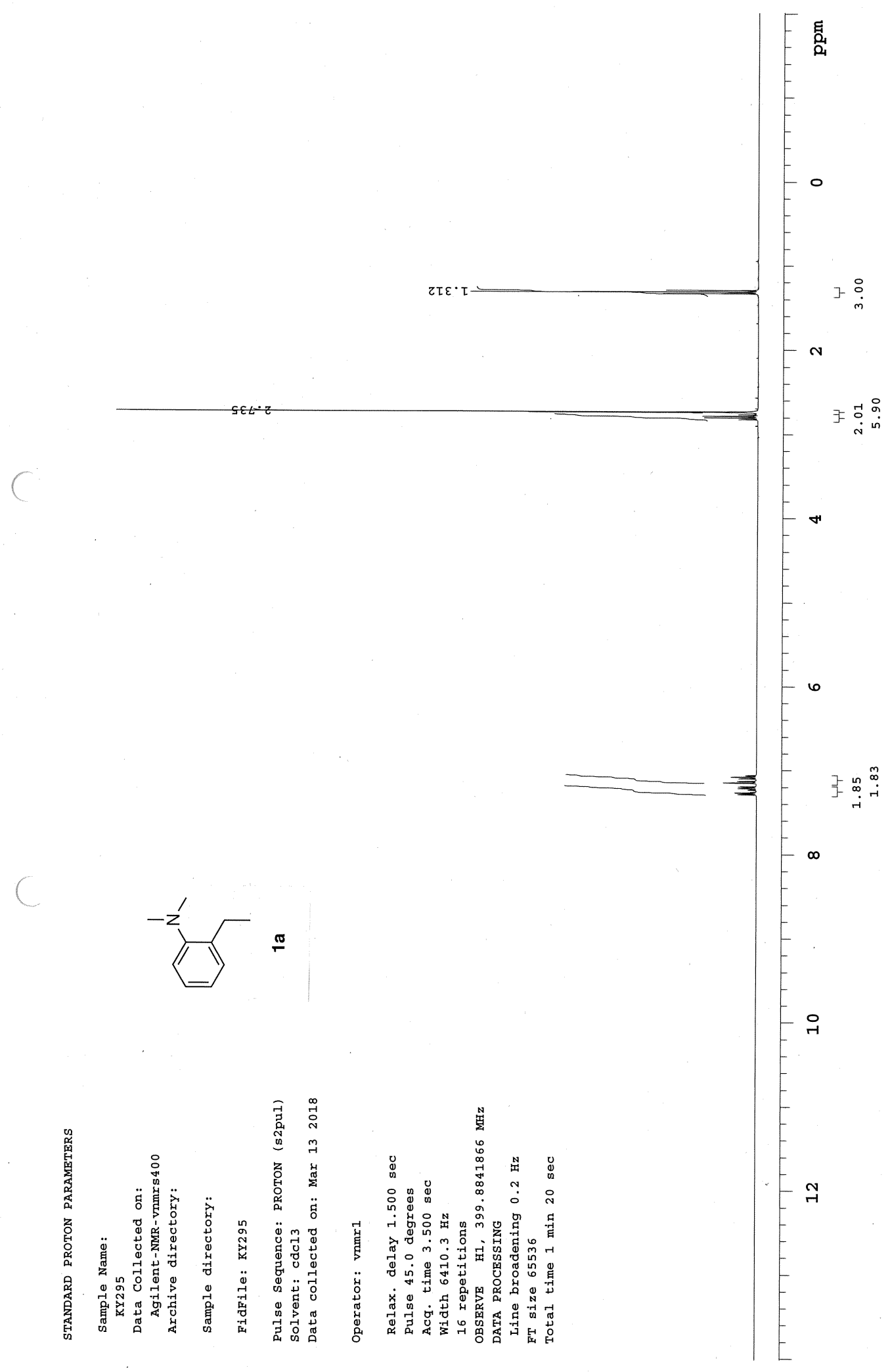

Figure S2. ${ }^{1} \mathrm{H}$ NMR Spectrum of $1 \mathbf{a}\left(400 \mathrm{MHz}, \mathrm{CDCl}_{3}\right)$ 


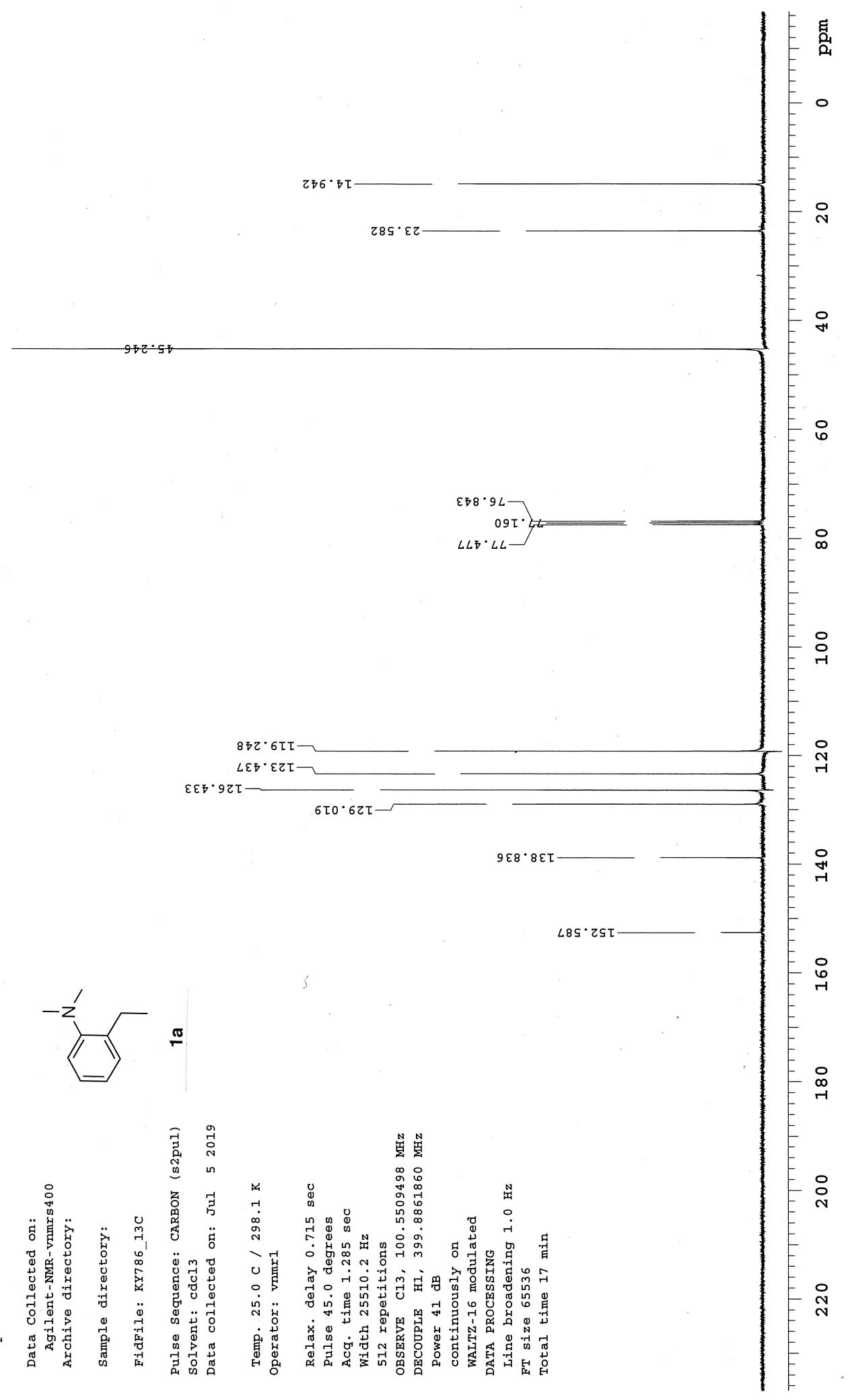

Figure S3. ${ }^{13} \mathrm{C}$ NMR Spectrum of $\mathbf{1 a}\left(101 \mathrm{MHz}, \mathrm{CDCl}_{3}\right)$ 


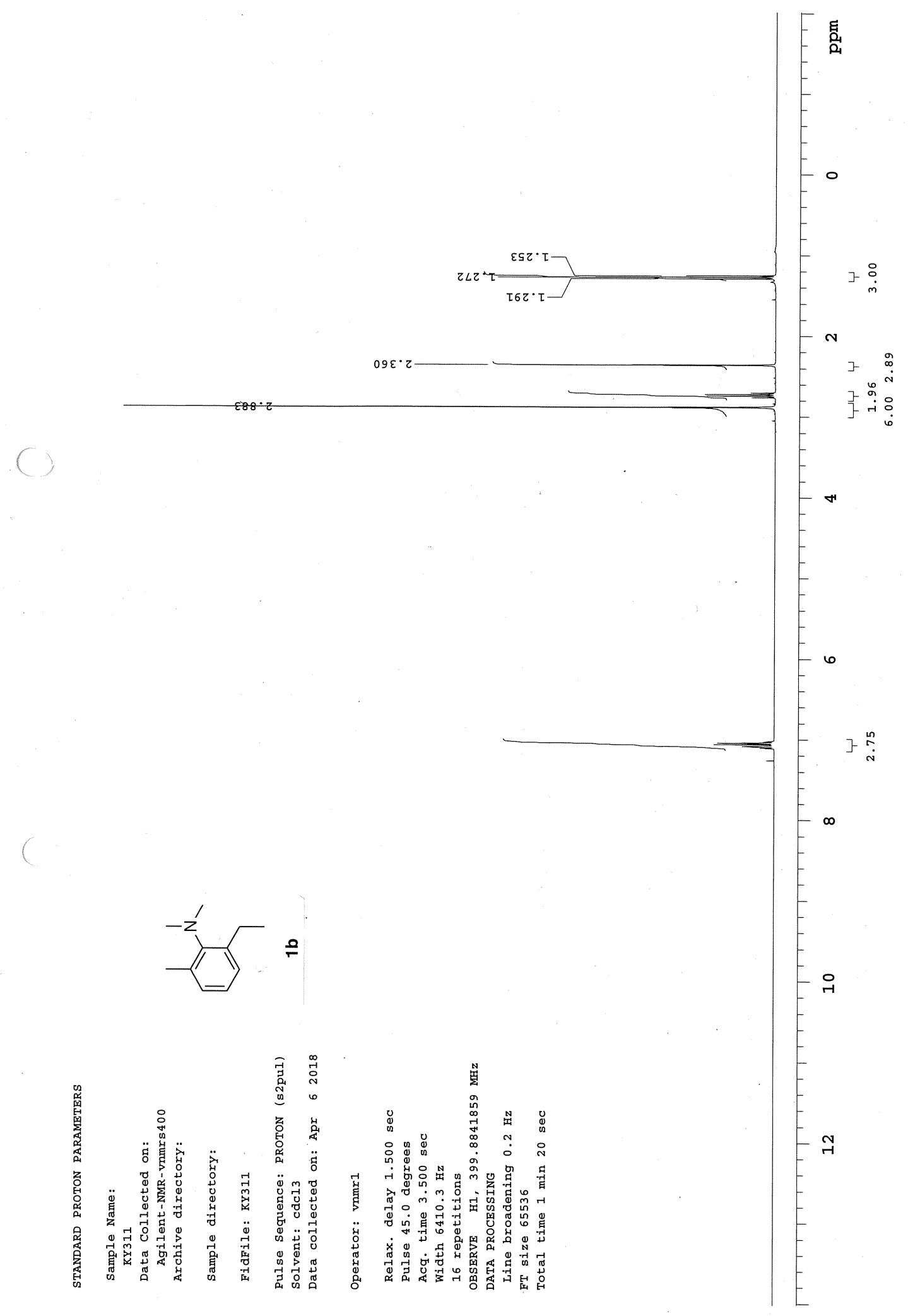

Figure S4. ${ }^{1} \mathrm{H}$ NMR Spectrum of $1 \mathbf{b}\left(400 \mathrm{MHz}, \mathrm{CDCl}_{3}\right)$ 


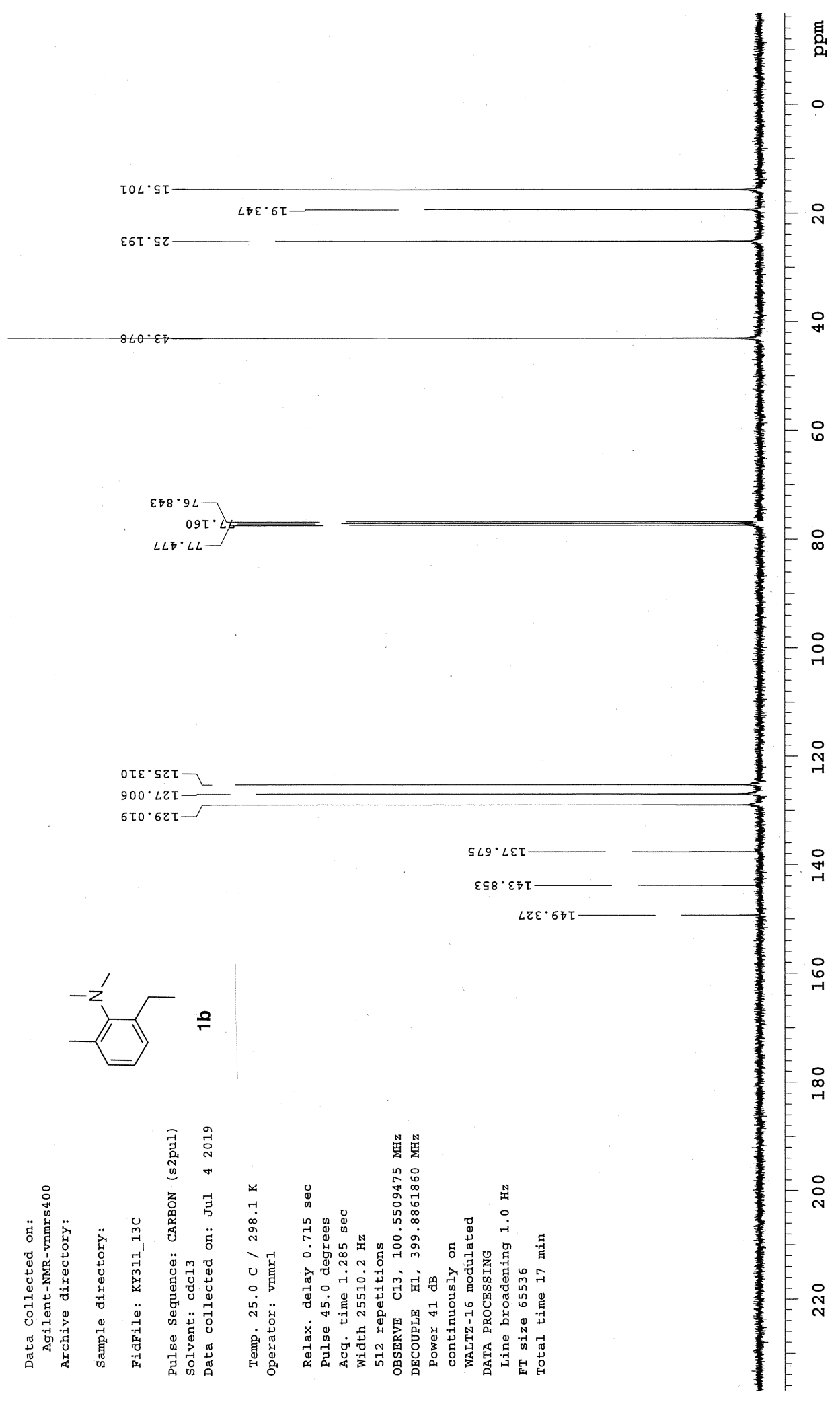

Figure S5. ${ }^{13} \mathrm{C}$ NMR Spectrum of $\mathbf{1 b}\left(101 \mathrm{MHz}, \mathrm{CDCl}_{3}\right)$ 


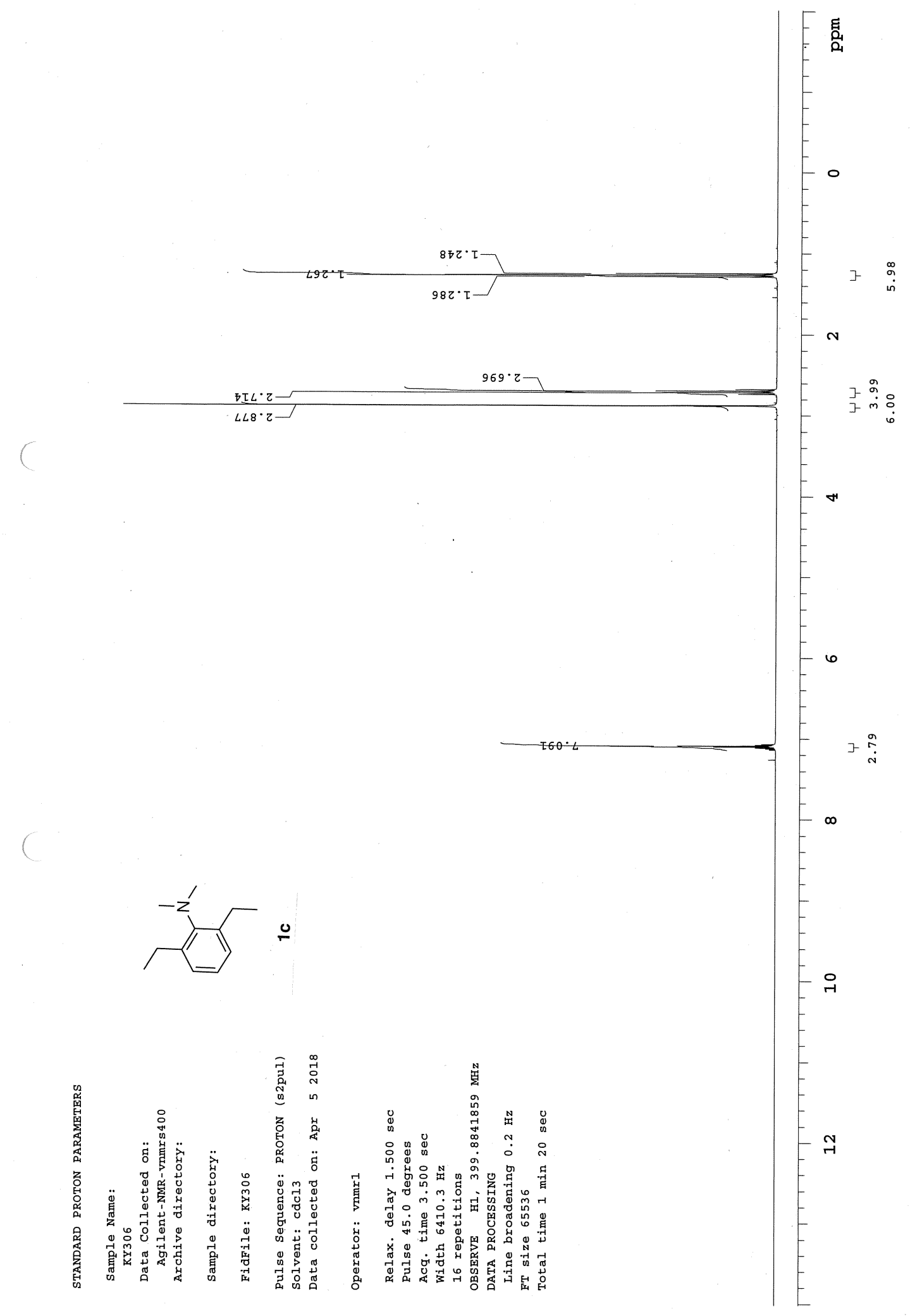

Figure S6. ${ }^{1} \mathrm{H}$ NMR Spectrum of 1 c $\left(400 \mathrm{MHz}, \mathrm{CDCl}_{3}\right)$ 


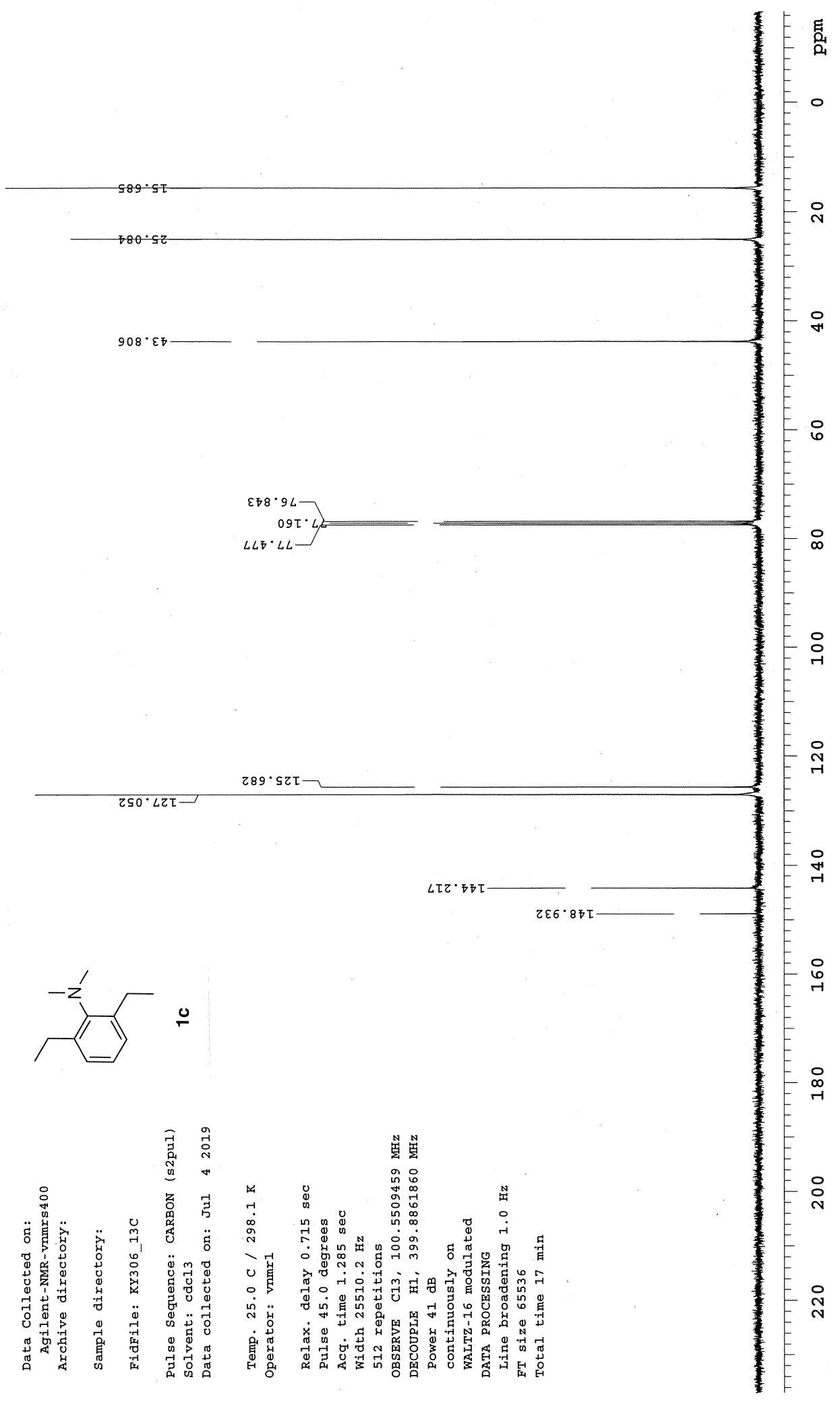

Figure S7. ${ }^{13} \mathrm{C}$ NMR Spectrum of $1 \mathbf{c}\left(101 \mathrm{MHz}, \mathrm{CDCl}_{3}\right)$ 


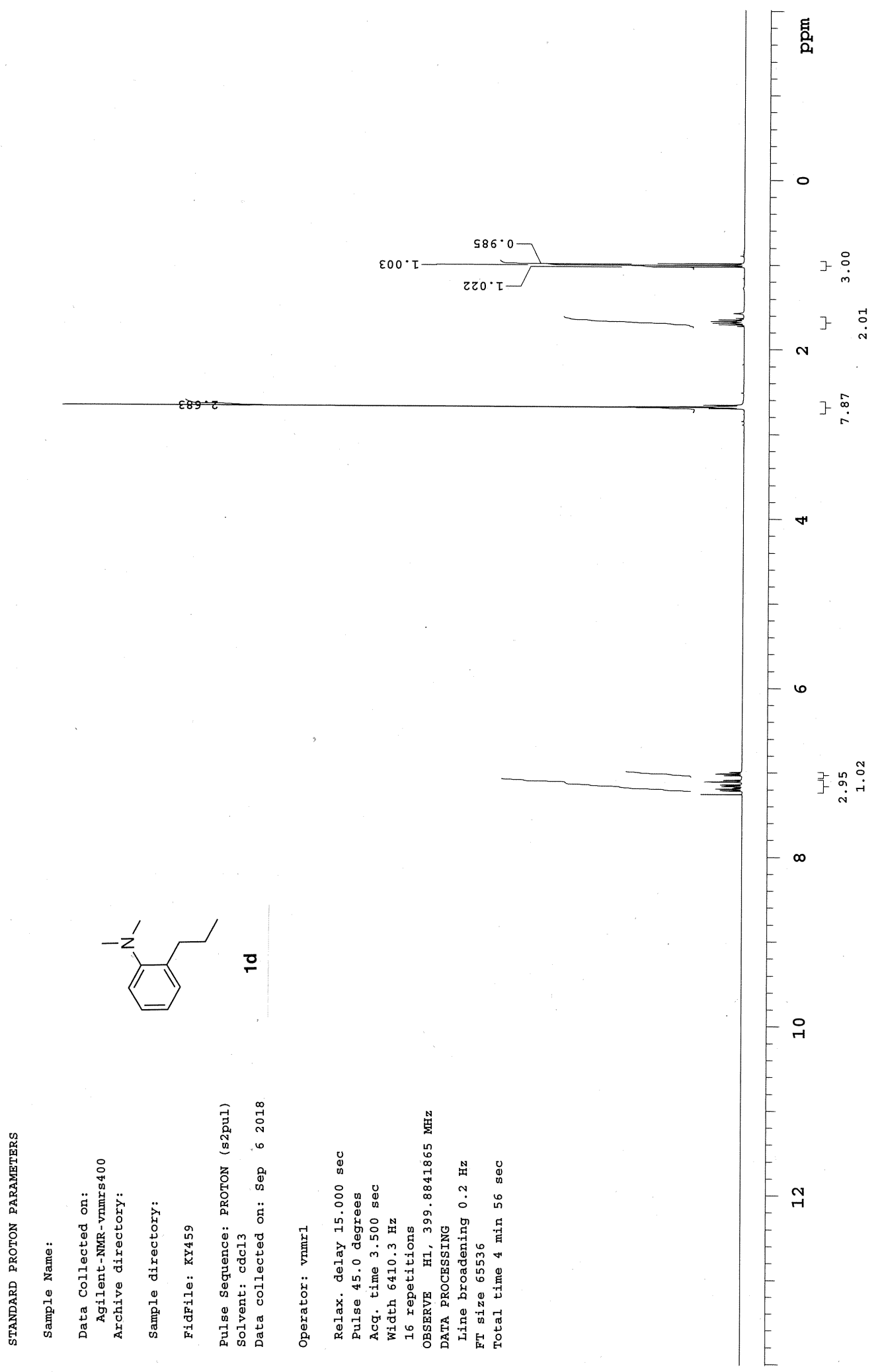

Figure S8. ${ }^{1} \mathrm{H}$ NMR Spectrum of $1 d\left(400 \mathrm{MHz}, \mathrm{CDCl}_{3}\right)$ 


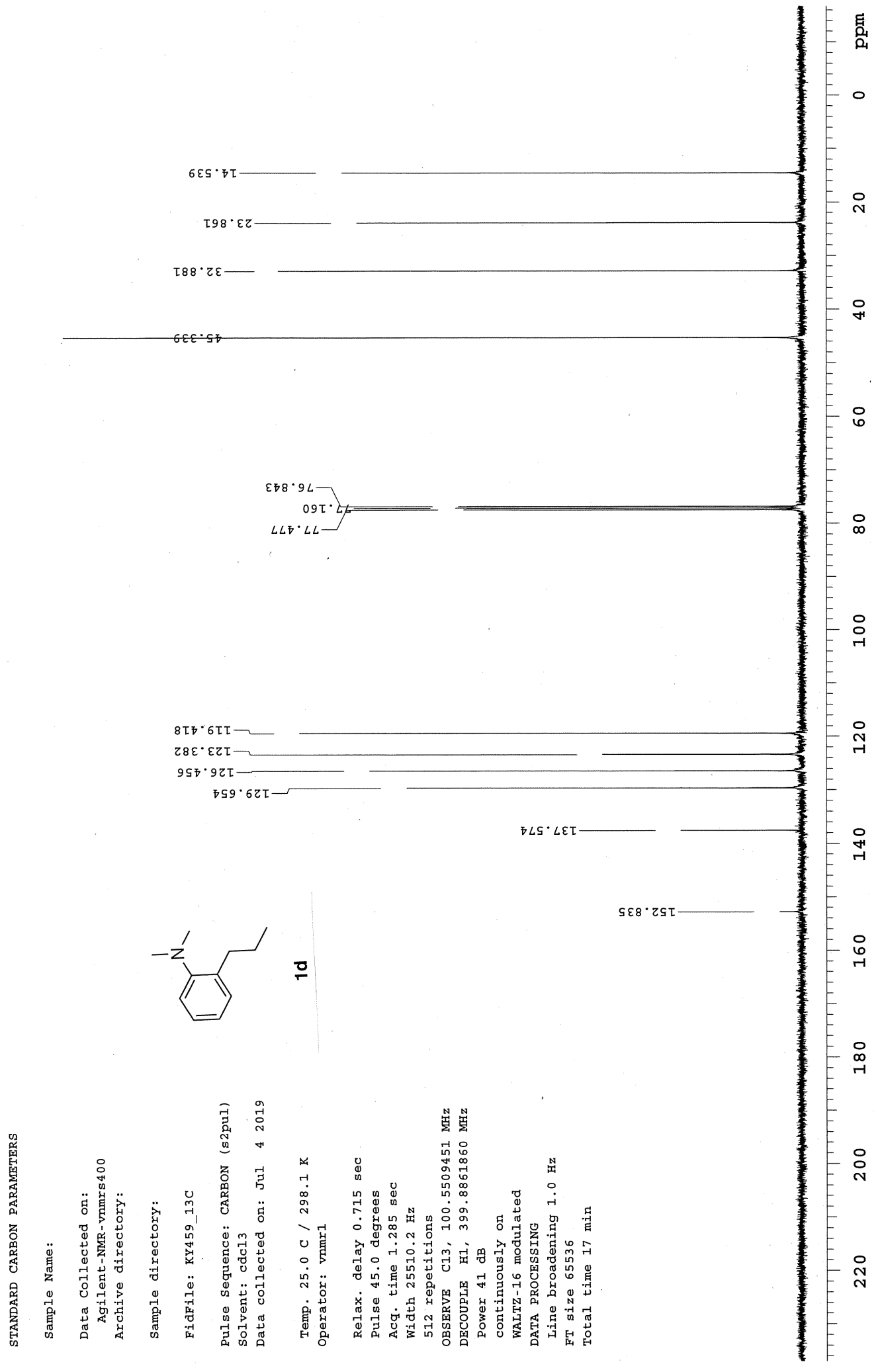

Figure S9. ${ }^{13} \mathrm{C}$ NMR Spectrum of $1 \mathbf{d}\left(101 \mathrm{MHz}, \mathrm{CDCl}_{3}\right)$ 


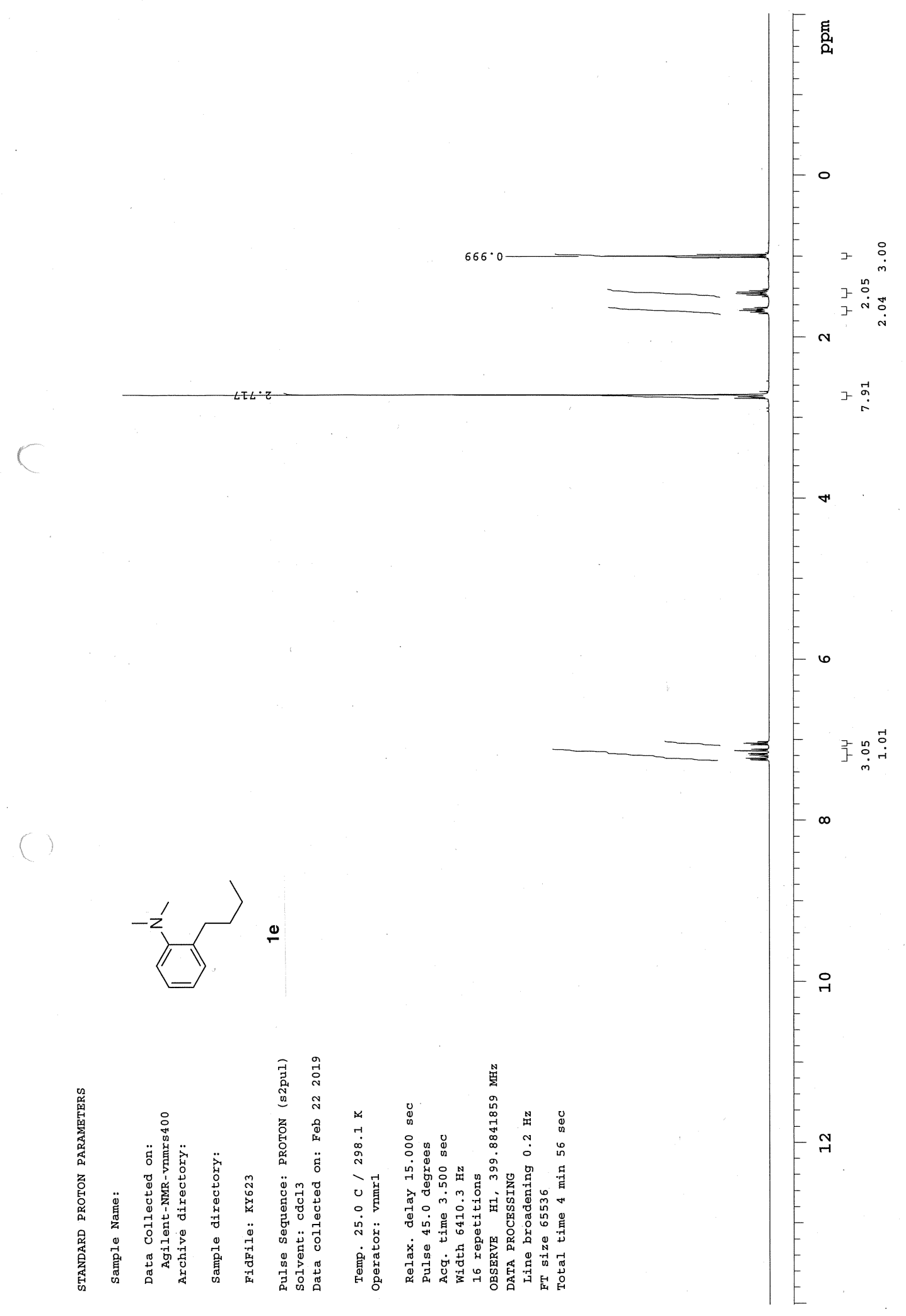

Figure S10. ${ }^{1} \mathrm{H}$ NMR Spectrum of 1 e $\left(400 \mathrm{MHz}, \mathrm{CDCl}_{3}\right)$ 


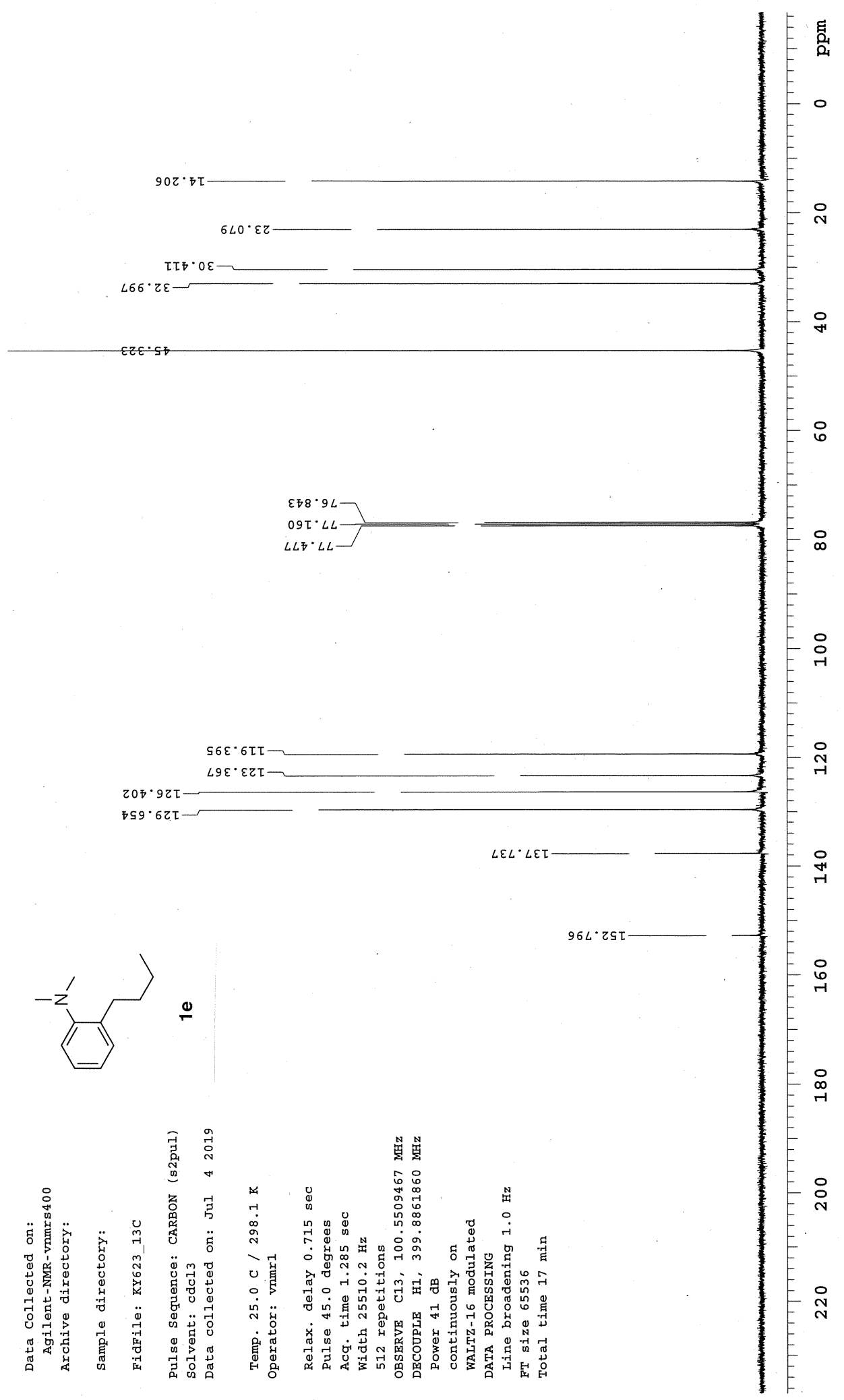

Figure S11. ${ }^{13} \mathrm{C}$ NMR Spectrum of 1 e $\left(101 \mathrm{MHz}, \mathrm{CDCl}_{3}\right)$ 


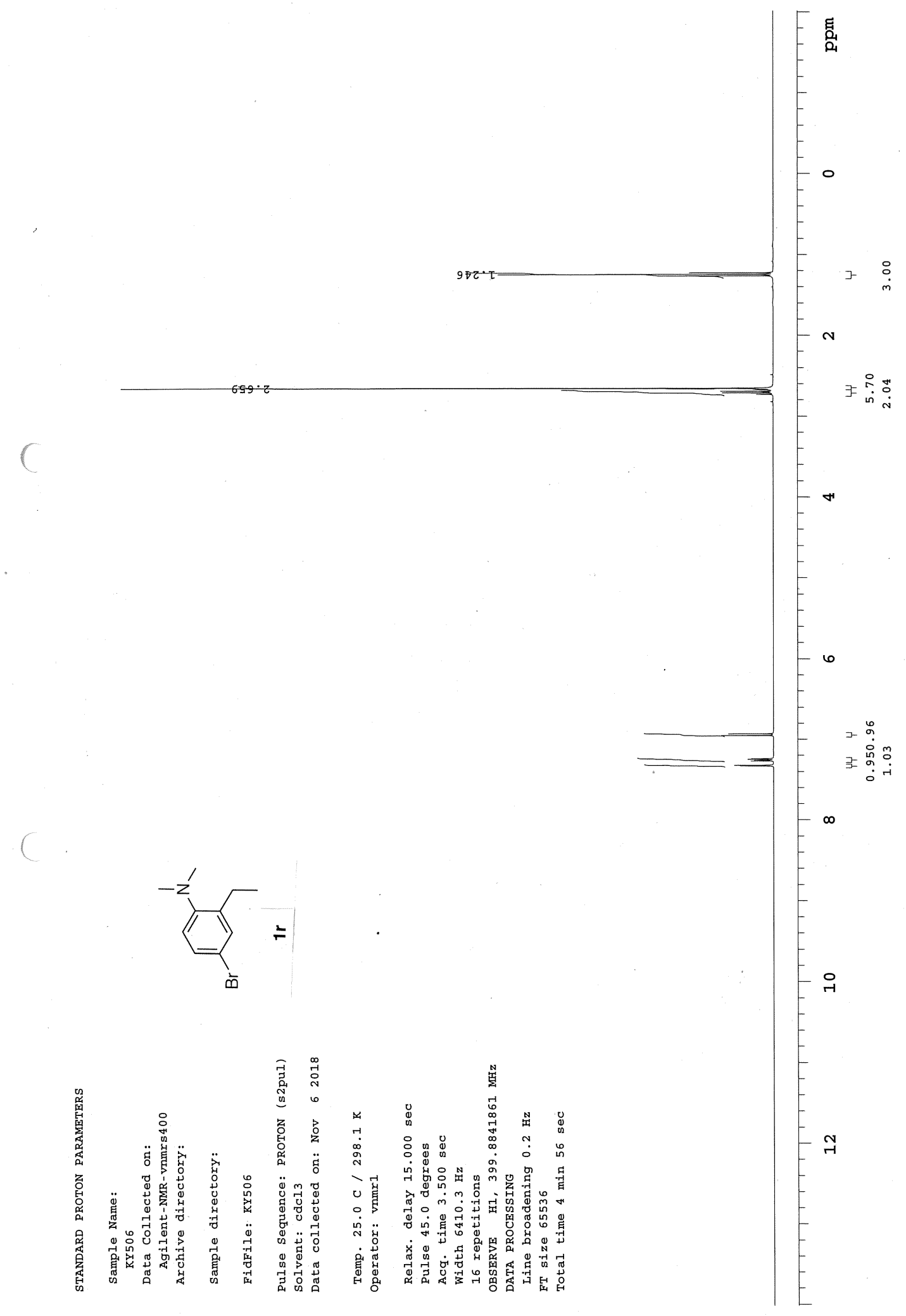

Figure S12. ${ }^{1} \mathrm{H}$ NMR Spectrum of $1 \mathbf{r}\left(400 \mathrm{MHz}, \mathrm{CDCl}_{3}\right)$ 


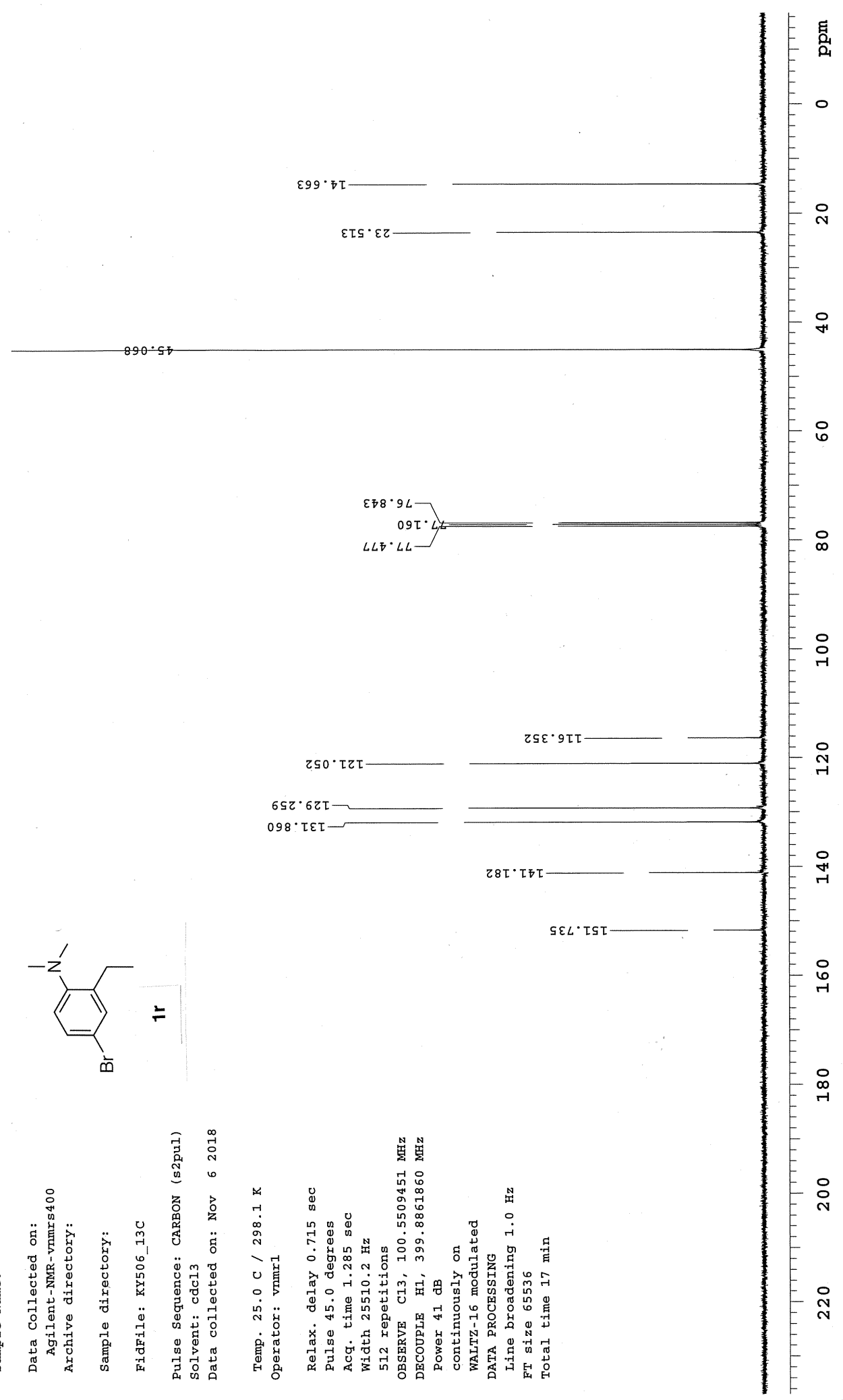

Figure S13. ${ }^{13} \mathrm{C}$ NMR Spectrum of $1 \mathbf{r}\left(101 \mathrm{MHz}, \mathrm{CDCl}_{3}\right)$ 


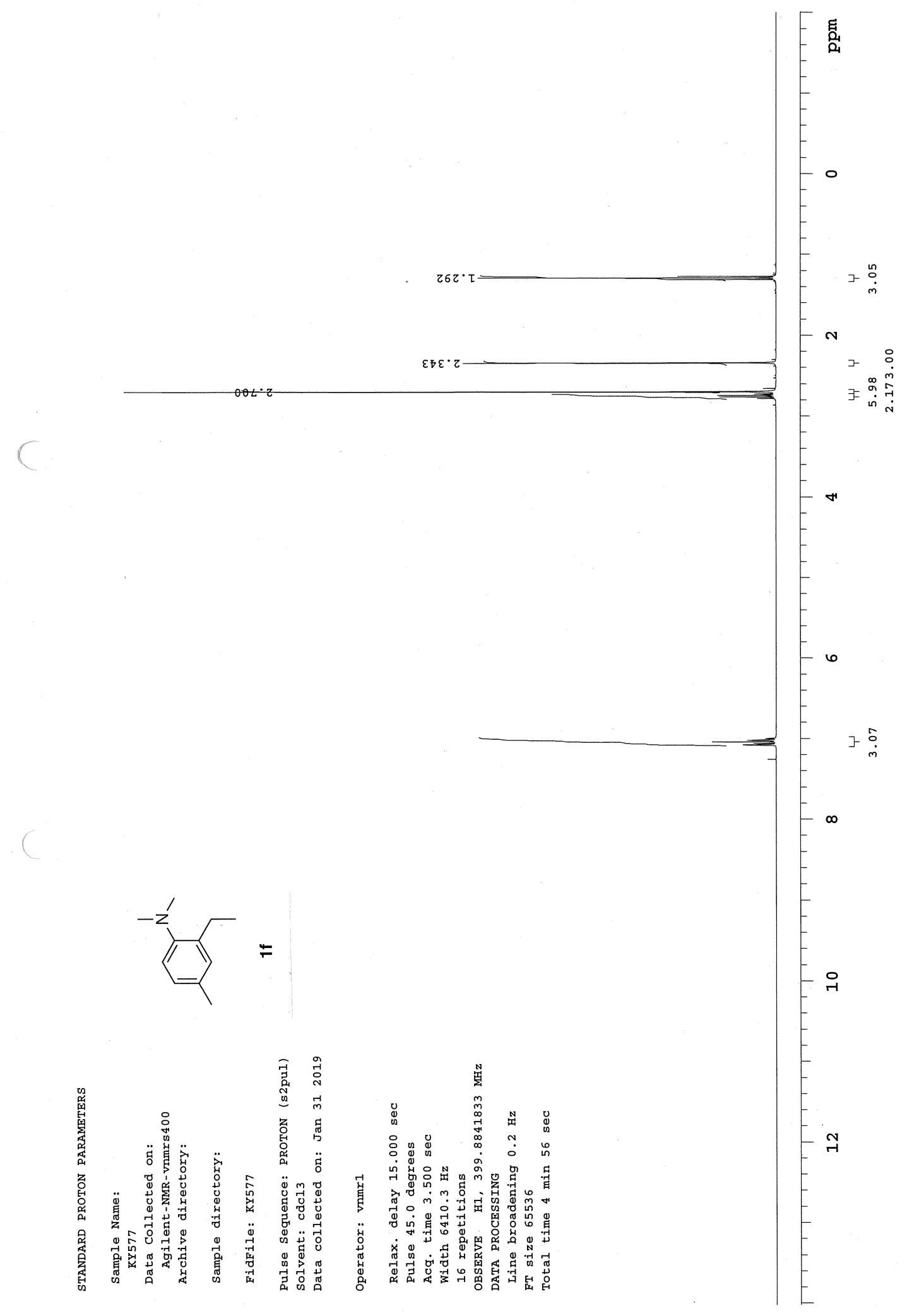

Figure S14. ${ }^{1} \mathrm{H}$ NMR Spectrum of $1 f\left(400 \mathrm{MHz}, \mathrm{CDCl}_{3}\right)$ 


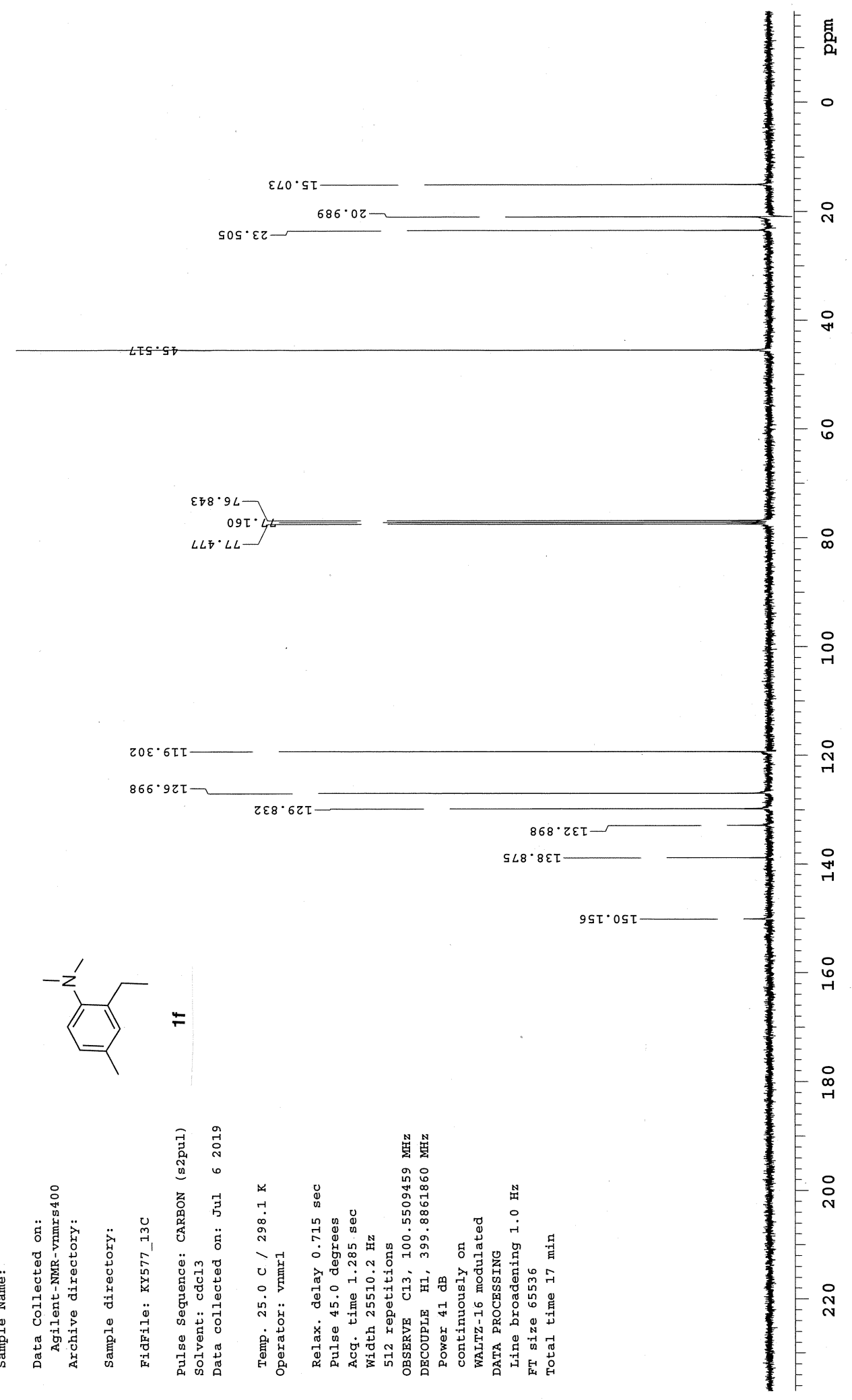

Figure S15. ${ }^{13} \mathrm{C}$ NMR Spectrum of $\mathbf{1 f}\left(101 \mathrm{MHz}, \mathrm{CDCl}_{3}\right)$ 


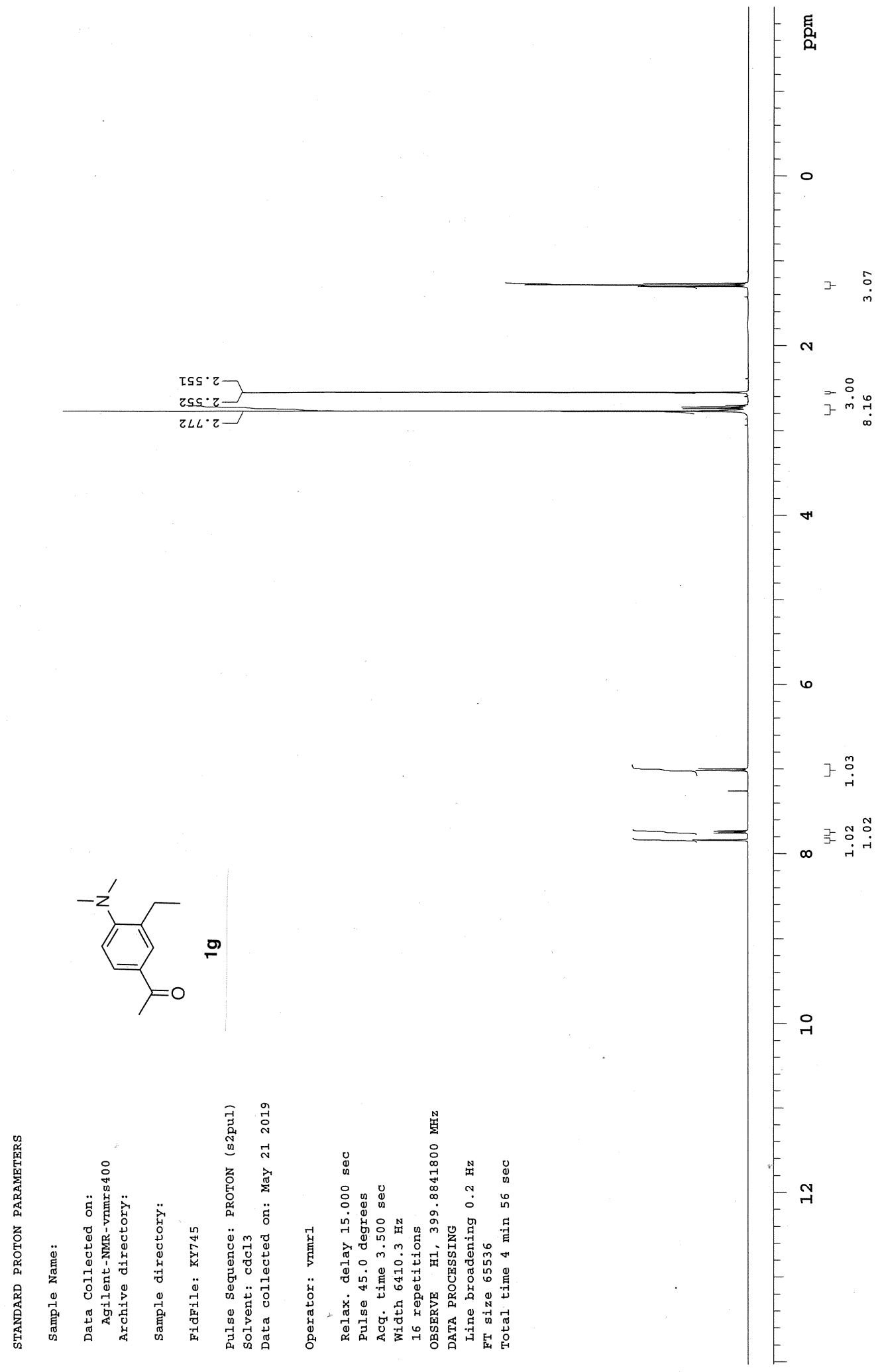

Figure S16. ${ }^{1} \mathrm{H}$ NMR Spectrum of 1 g $\left(400 \mathrm{MHz}, \mathrm{CDCl}_{3}\right)$ 


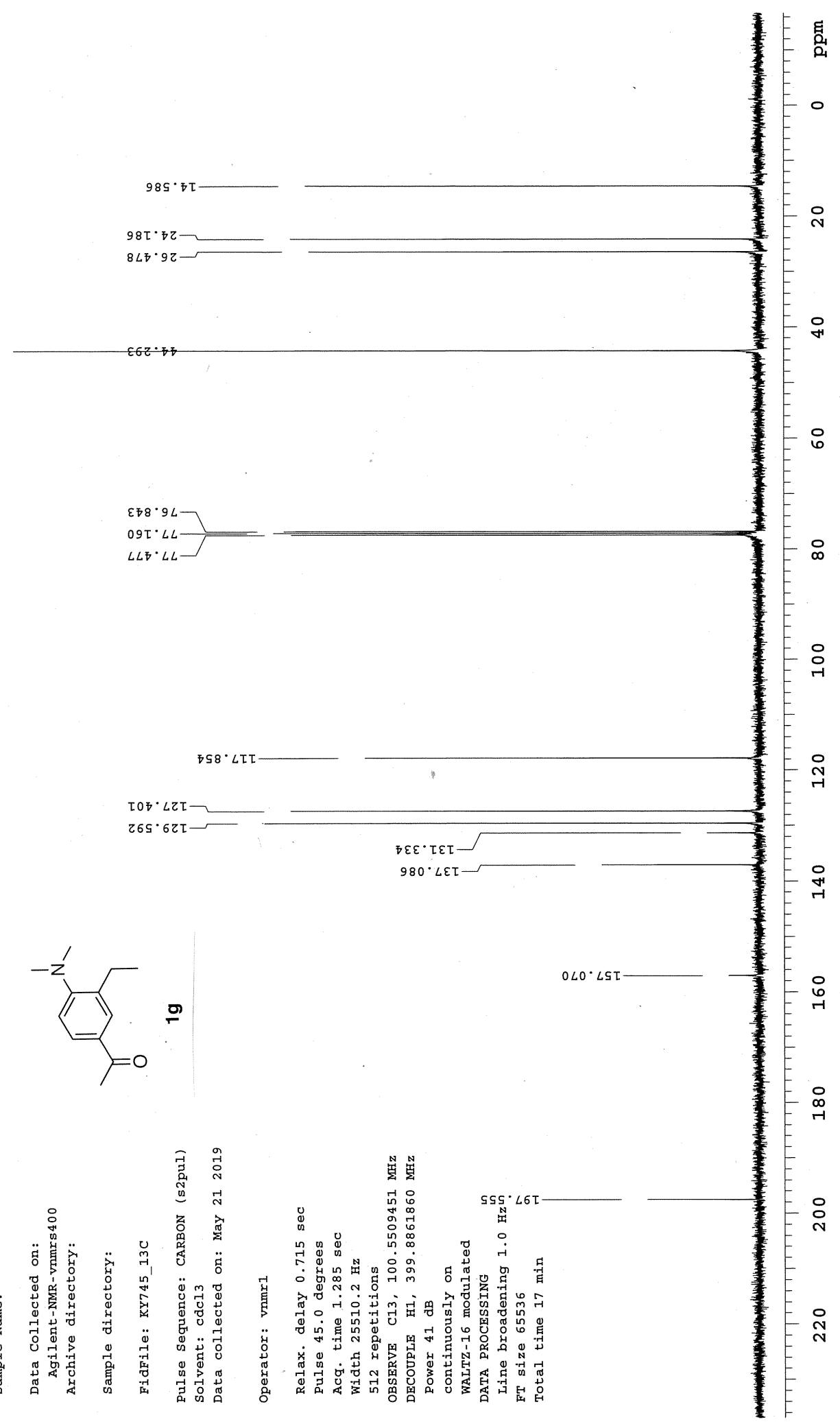

Figure S17. ${ }^{13} \mathrm{C}$ NMR Spectrum of 1 g $\left(101 \mathrm{MHz}, \mathrm{CDCl}_{3}\right)$ 


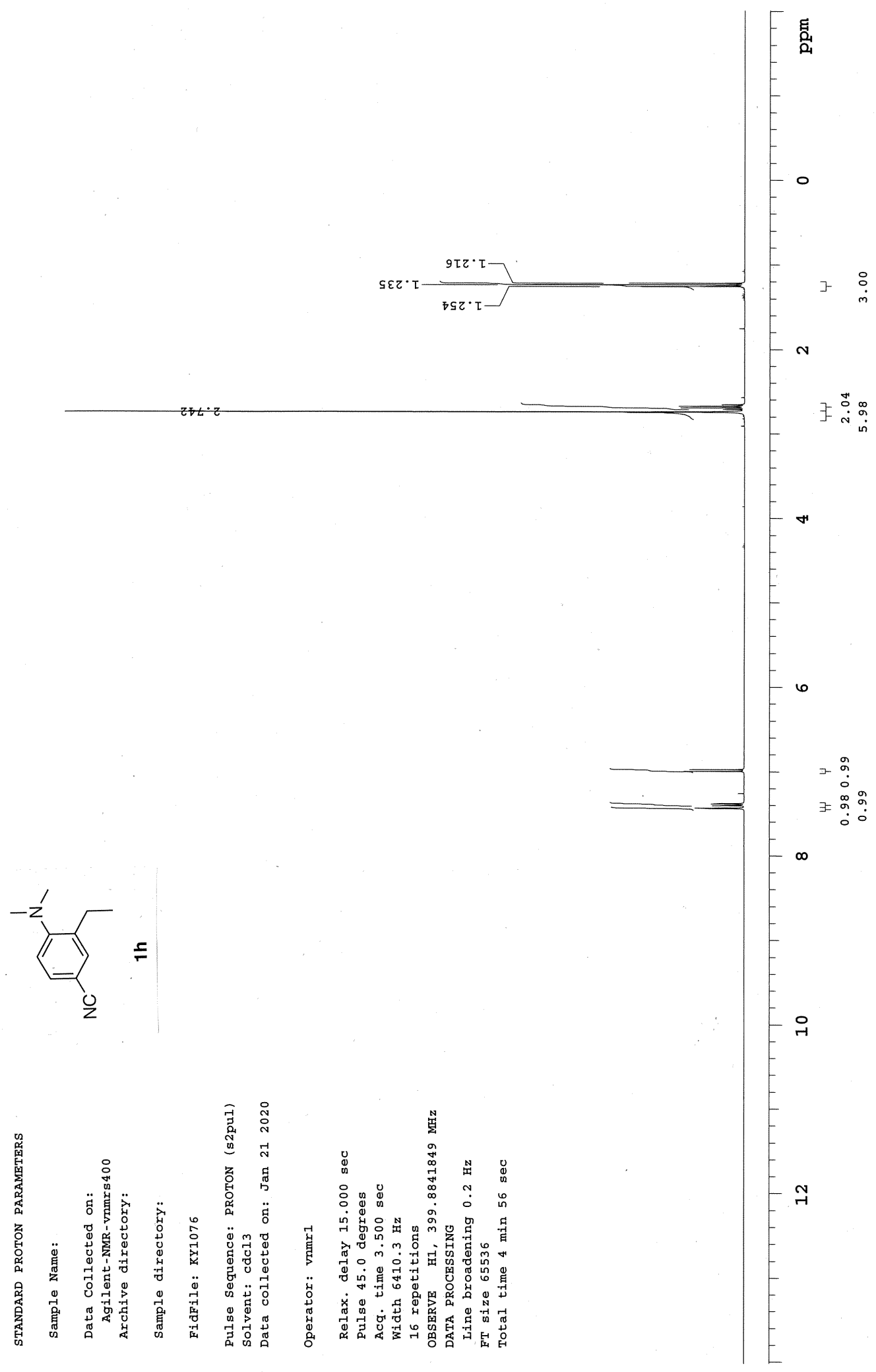

Figure S18. ${ }^{1} \mathrm{H}$ NMR Spectrum of $\mathbf{1 h}\left(400 \mathrm{MHz}, \mathrm{CDCl}_{3}\right)$ 


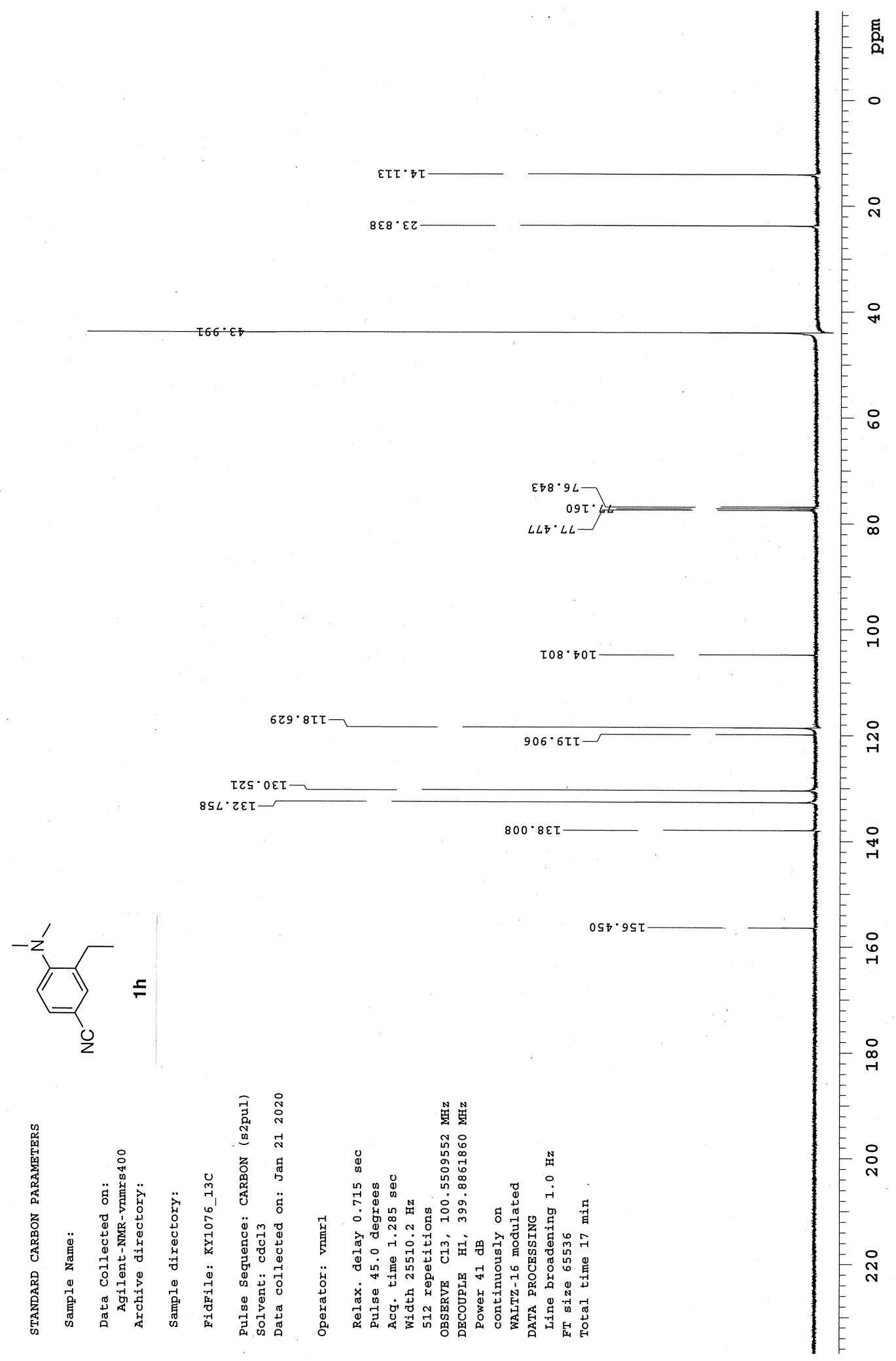

Figure S19. ${ }^{13} \mathrm{C}$ NMR Spectrum of $\mathbf{1 h}\left(101 \mathrm{MHz}, \mathrm{CDCl}_{3}\right)$ 


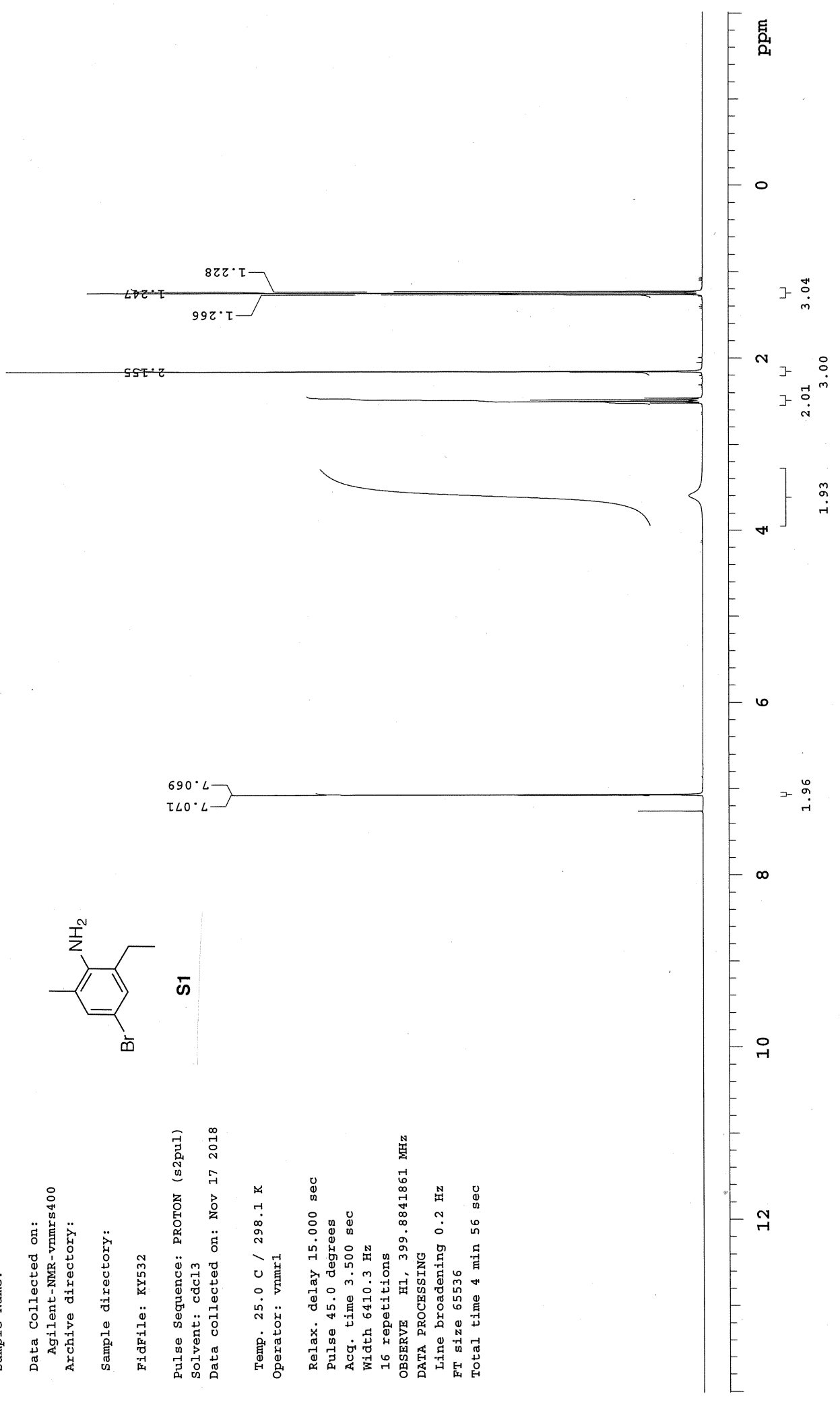

Figure S20. ${ }^{1} \mathrm{H}$ NMR Spectrum of S1 $\left(400 \mathrm{MHz}, \mathrm{CDCl}_{3}\right)$ 


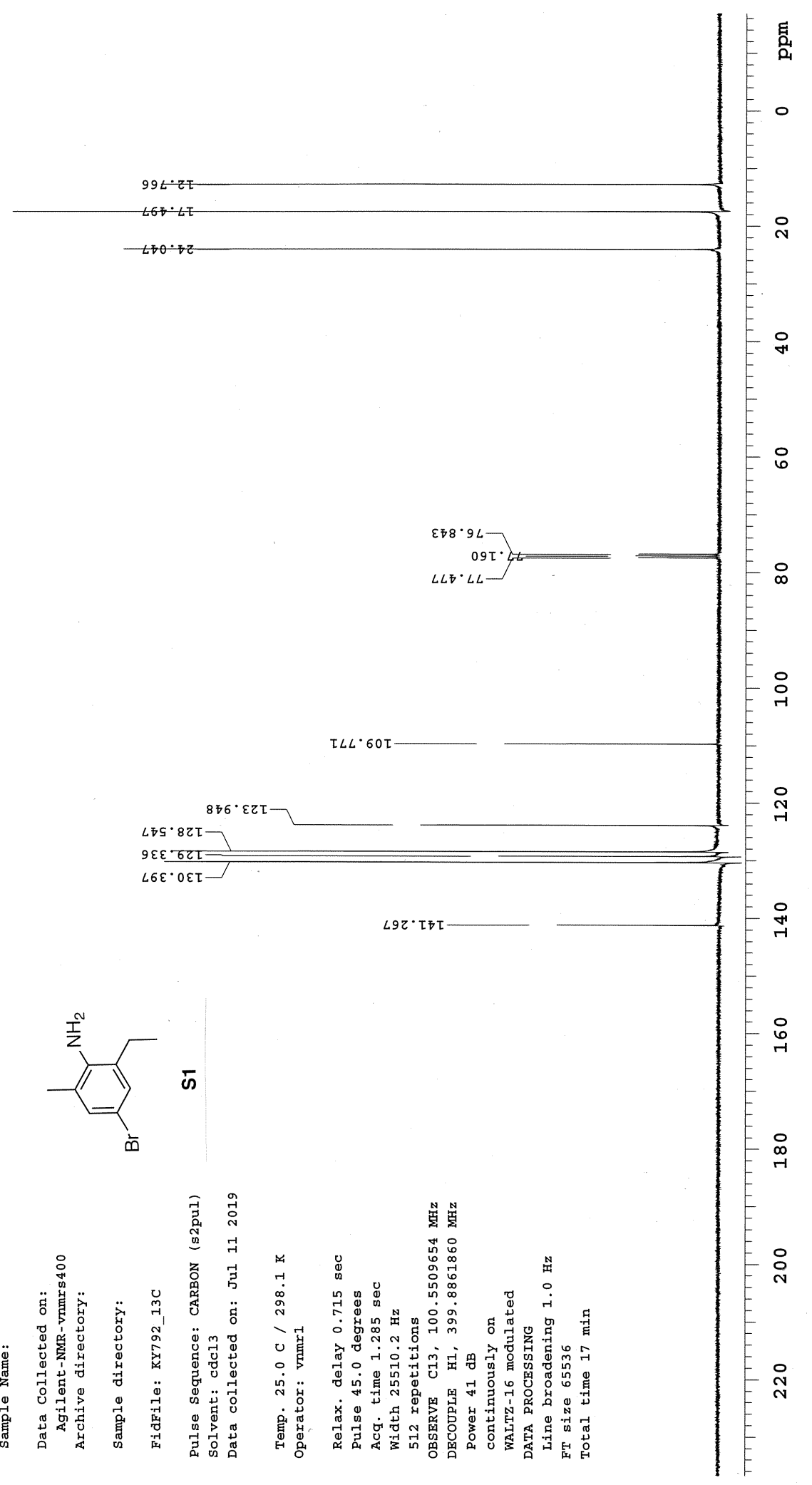

Figure S21. ${ }^{13} \mathrm{C}$ NMR Spectrum of $\mathbf{S 1}\left(101 \mathrm{MHz}, \mathrm{CDCl}_{3}\right)$ 


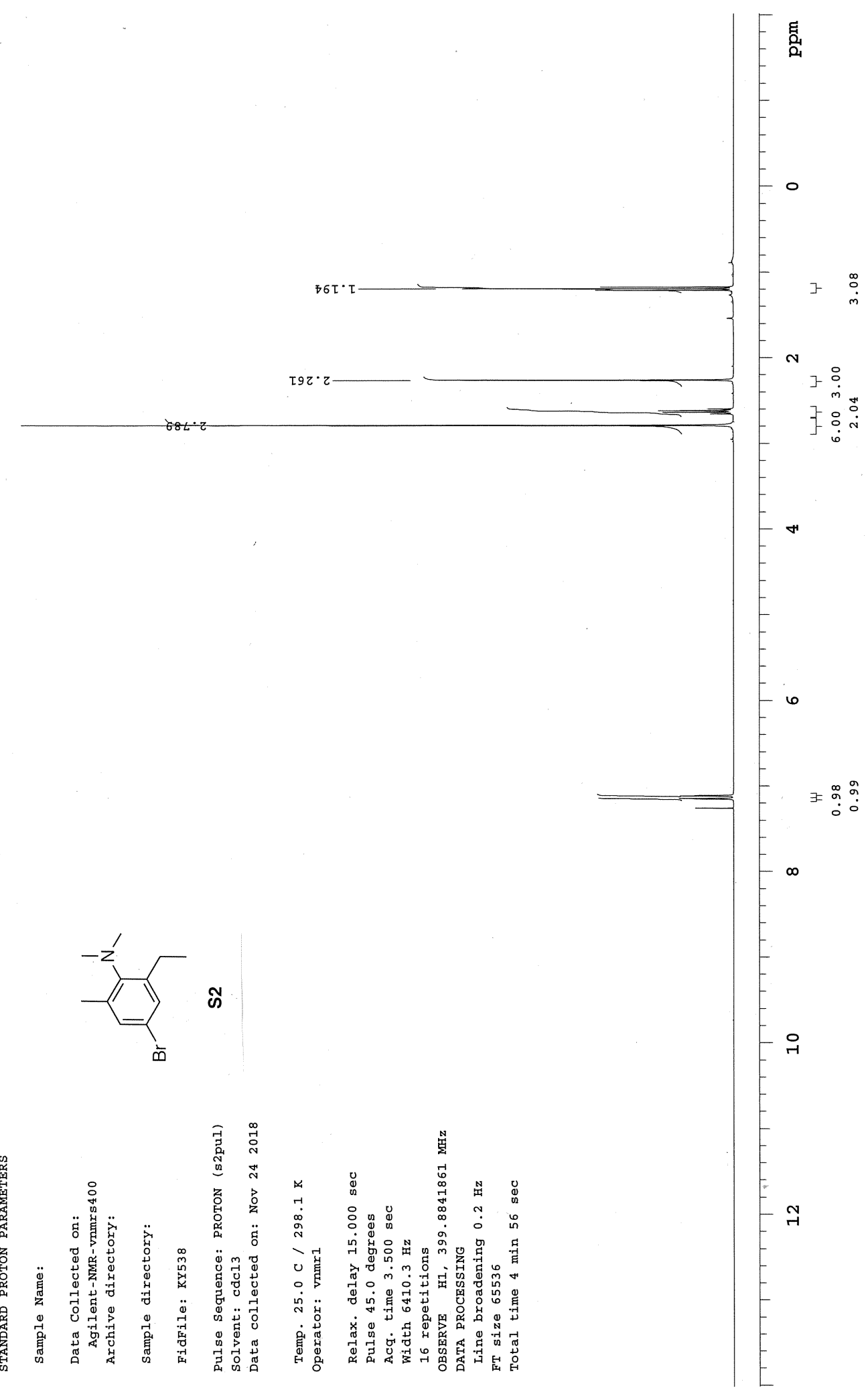

Figure S22. ${ }^{1} \mathrm{H}$ NMR Spectrum of $\mathbf{S 2}\left(400 \mathrm{MHz}, \mathrm{CDCl}_{3}\right)$ 


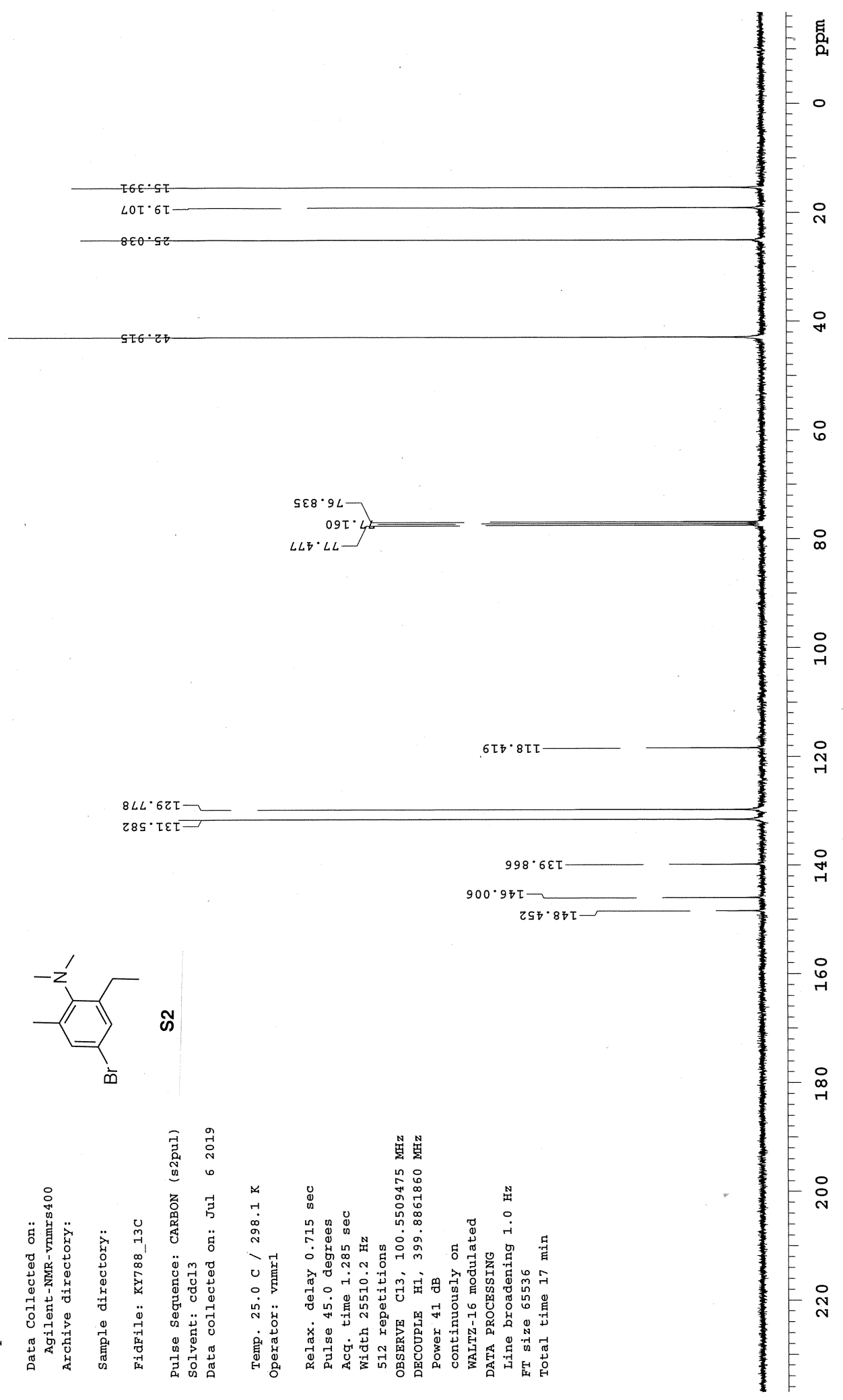

Figure S23. ${ }^{13} \mathrm{C}$ NMR Spectrum of $\mathbf{S 2}\left(101 \mathrm{MHz}, \mathrm{CDCl}_{3}\right)$ 


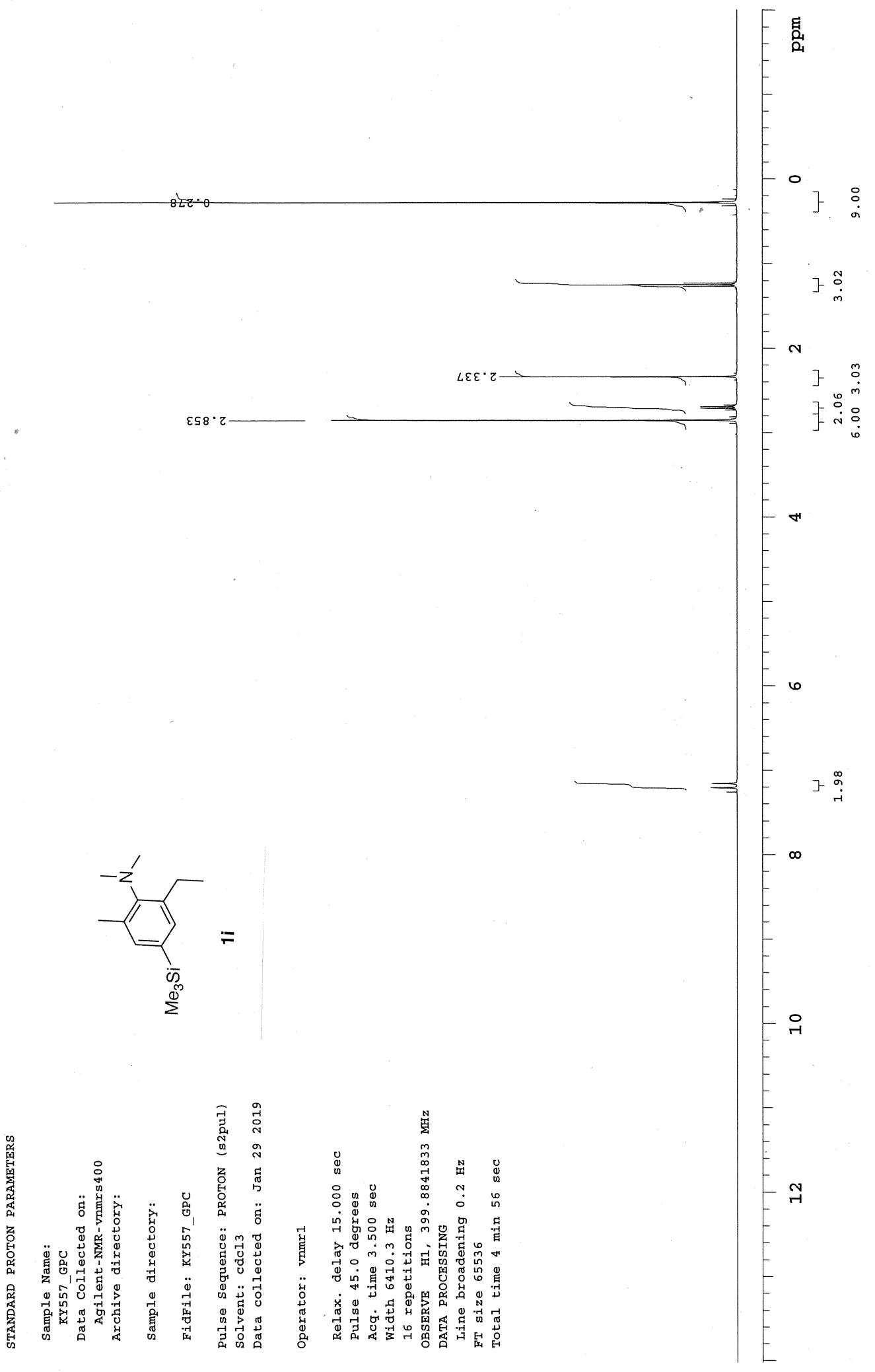

Figure S24. ${ }^{1} \mathrm{H}$ NMR Spectrum of $1 \mathbf{i}\left(400 \mathrm{MHz}, \mathrm{CDCl}_{3}\right)$ 


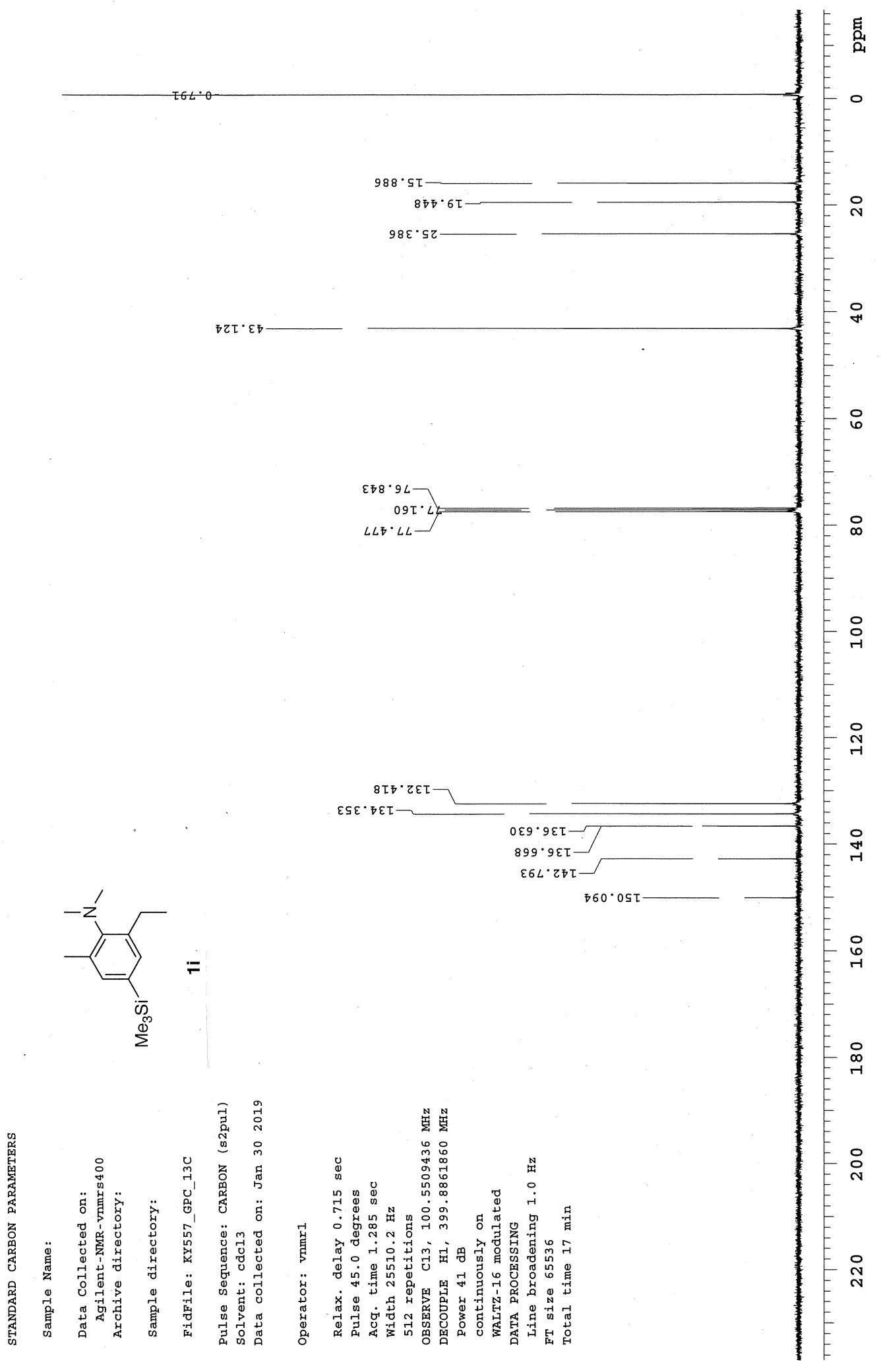

Figure S25. ${ }^{13} \mathrm{C}$ NMR Spectrum of $1 \mathbf{i}\left(101 \mathrm{MHz}, \mathrm{CDCl}_{3}\right)$ 


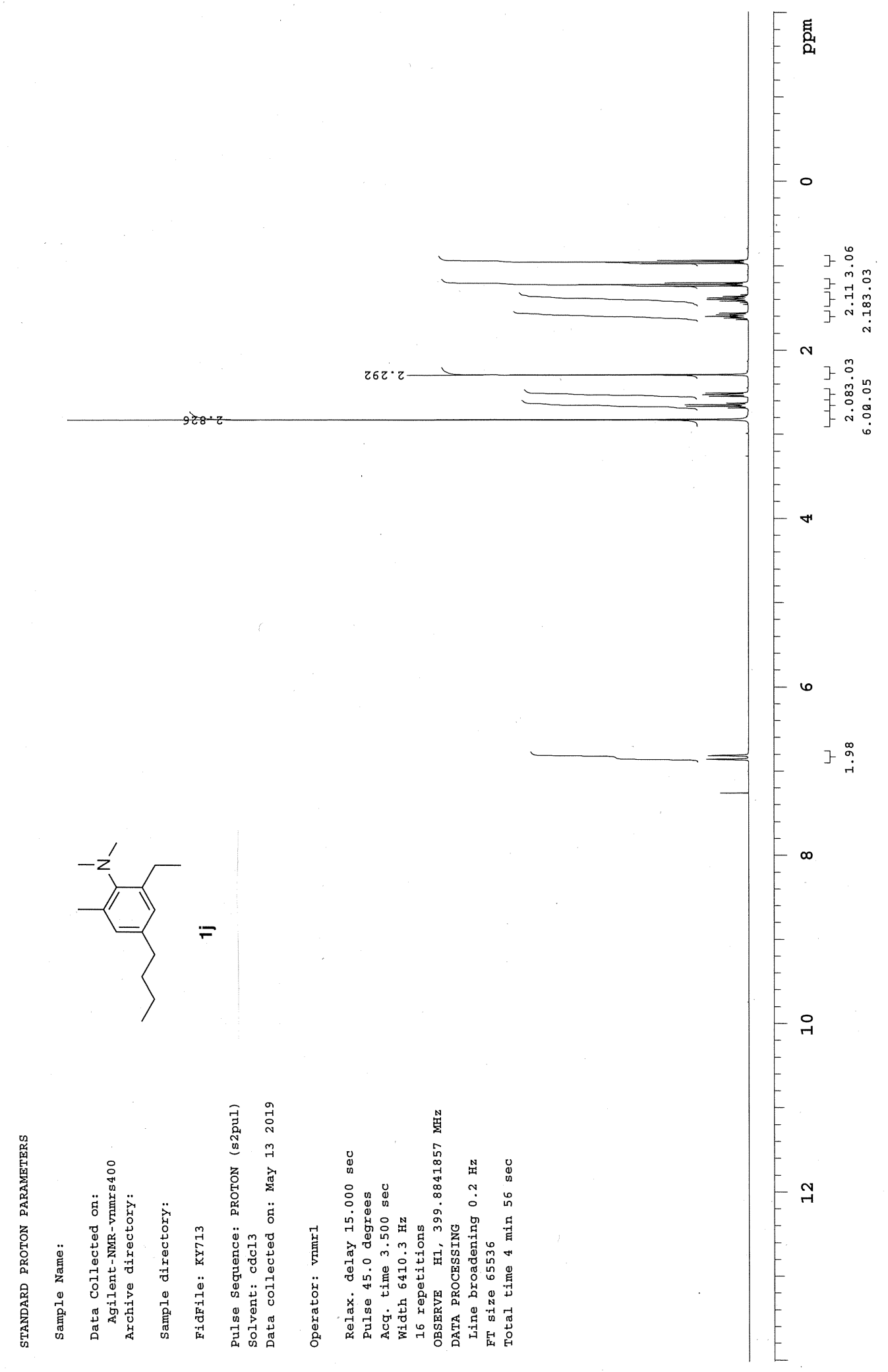

Figure S26. ${ }^{1} \mathrm{H}$ NMR Spectrum of $\mathbf{1 j}\left(400 \mathrm{MHz}, \mathrm{CDCl}_{3}\right)$ 


$$
\pm
$$




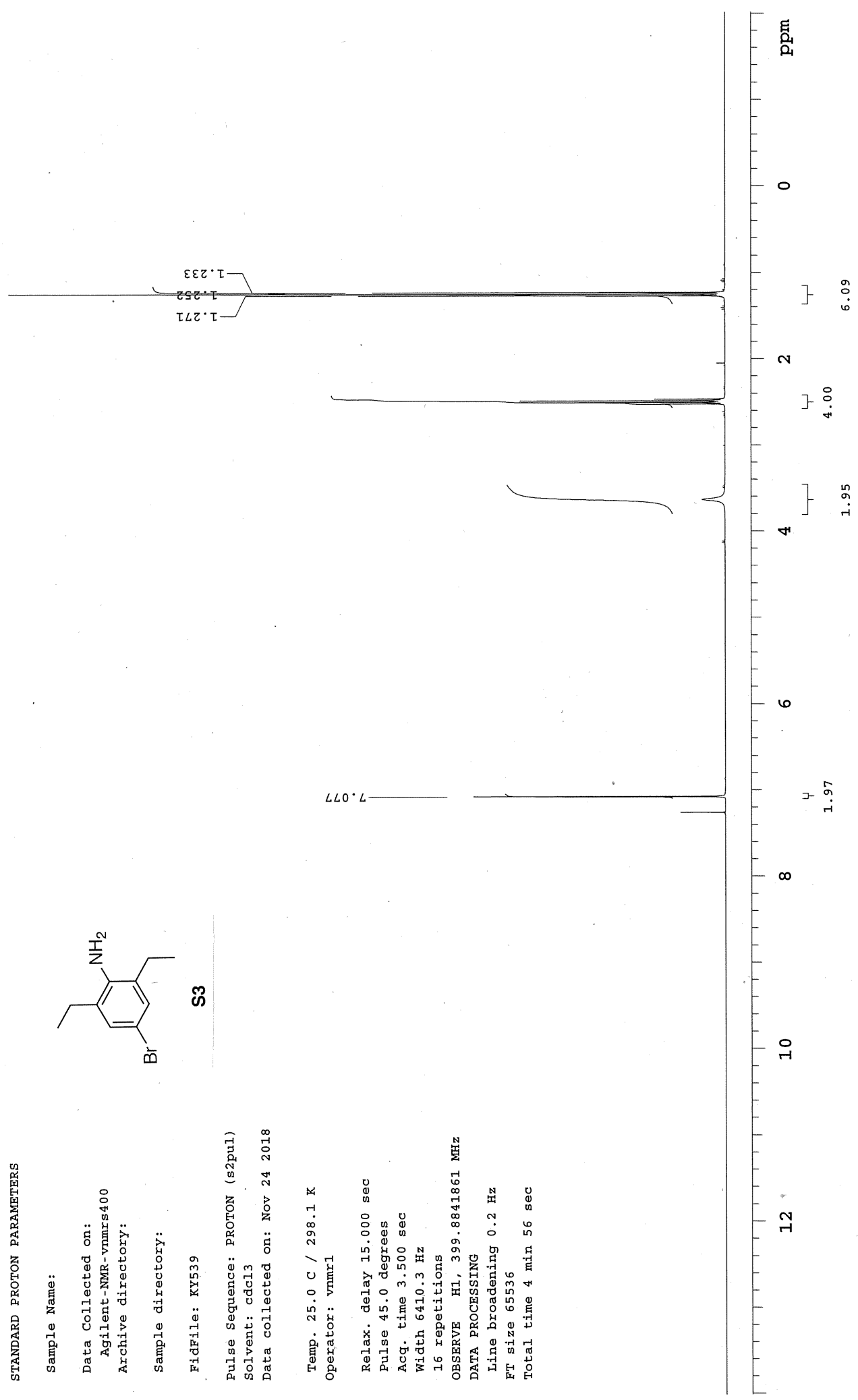

Figure S28. ${ }^{1} \mathrm{H}$ NMR Spectrum of $\mathbf{S 3}\left(400 \mathrm{MHz}, \mathrm{CDCl}_{3}\right)$ 


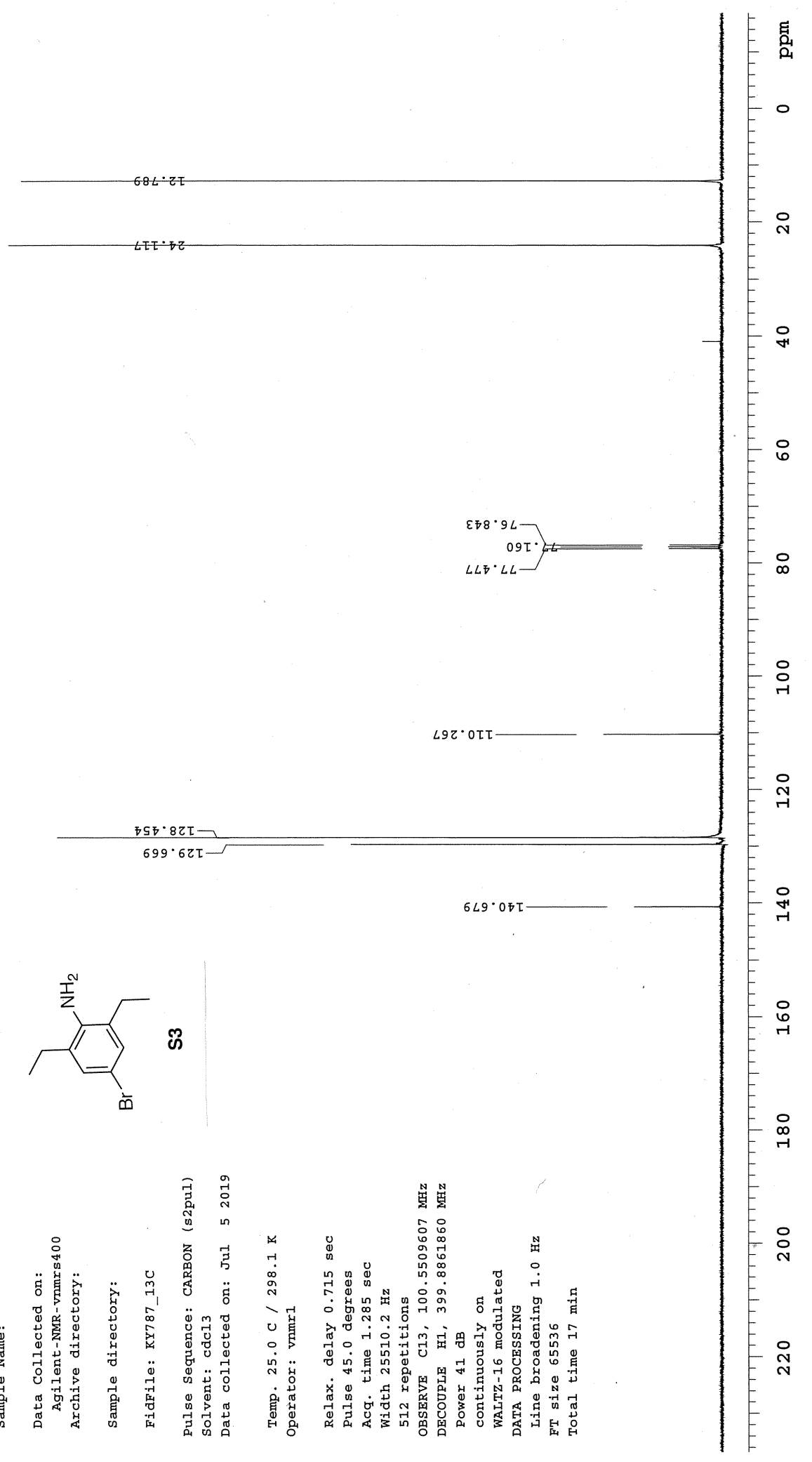

Figure S29. ${ }^{13} \mathrm{C}$ NMR Spectrum of $\mathbf{S 3}\left(101 \mathrm{MHz}, \mathrm{CDCl}_{3}\right)$ 


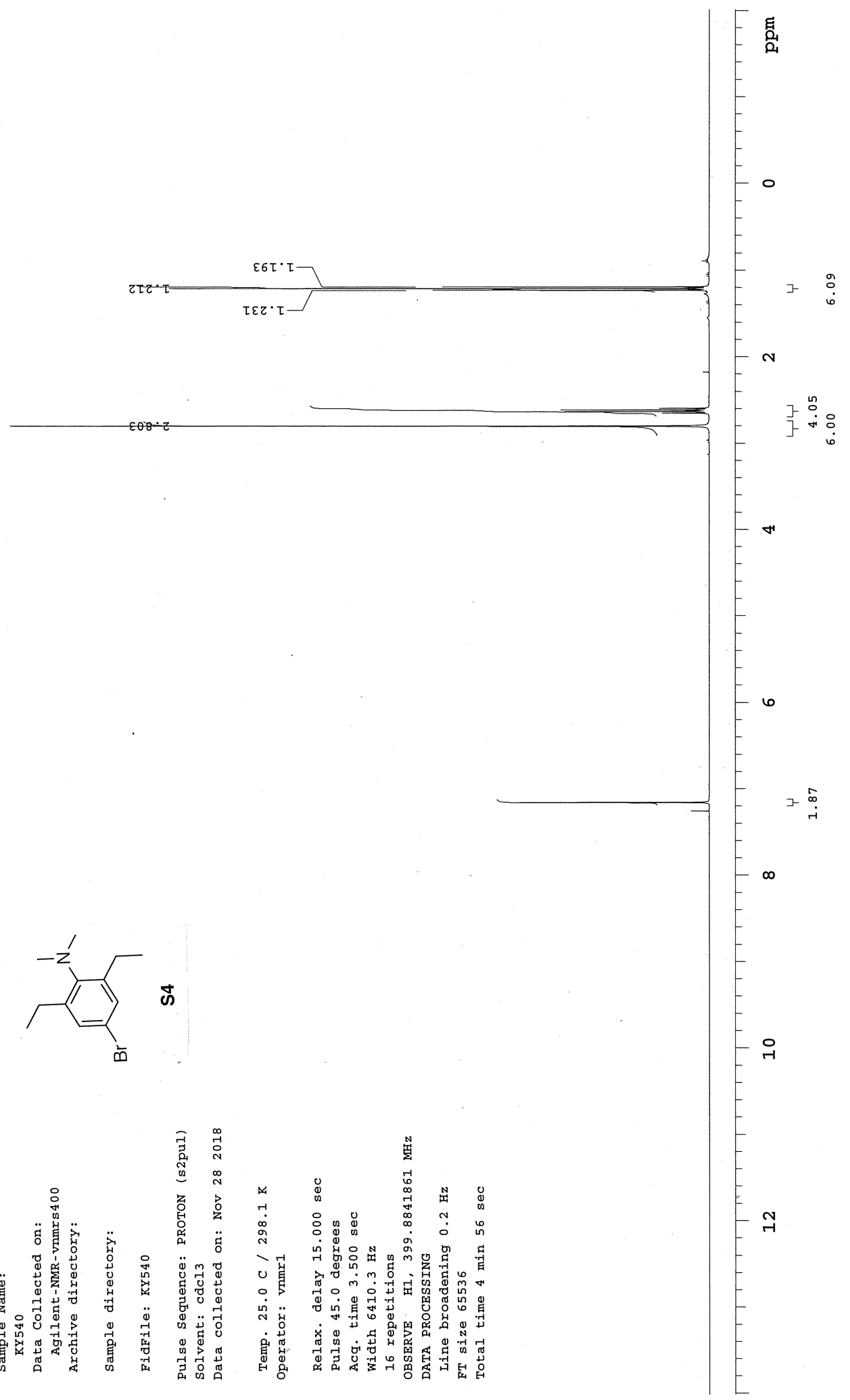

Figure S30. ${ }^{1} \mathrm{H}$ NMR Spectrum of $\mathbf{S 4}\left(400 \mathrm{MHz}, \mathrm{CDCl}_{3}\right)$ 


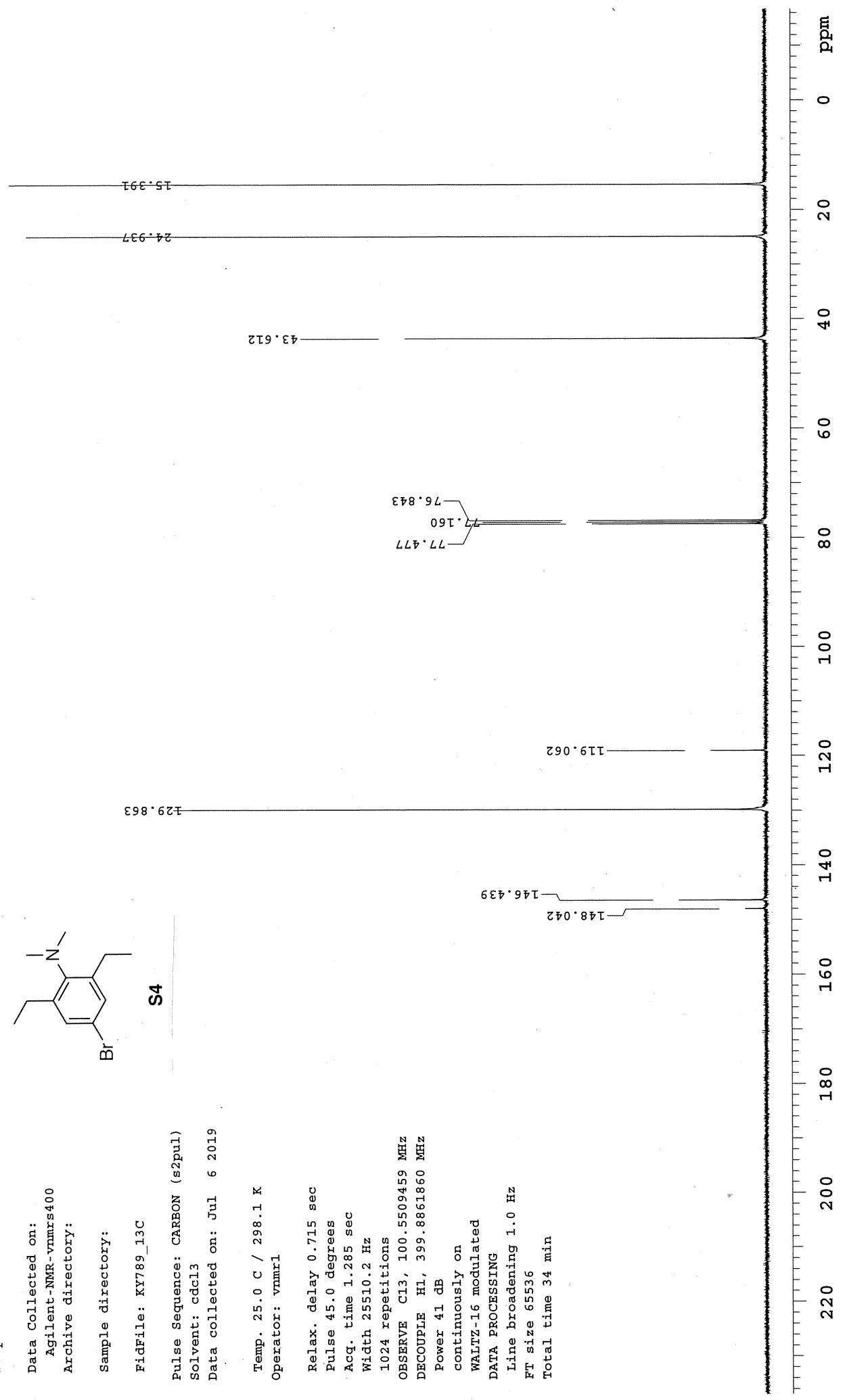

Figure S31. ${ }^{13} \mathrm{C}$ NMR Spectrum of $\mathbf{S 4}\left(101 \mathrm{MHz}, \mathrm{CDCl}_{3}\right)$ 


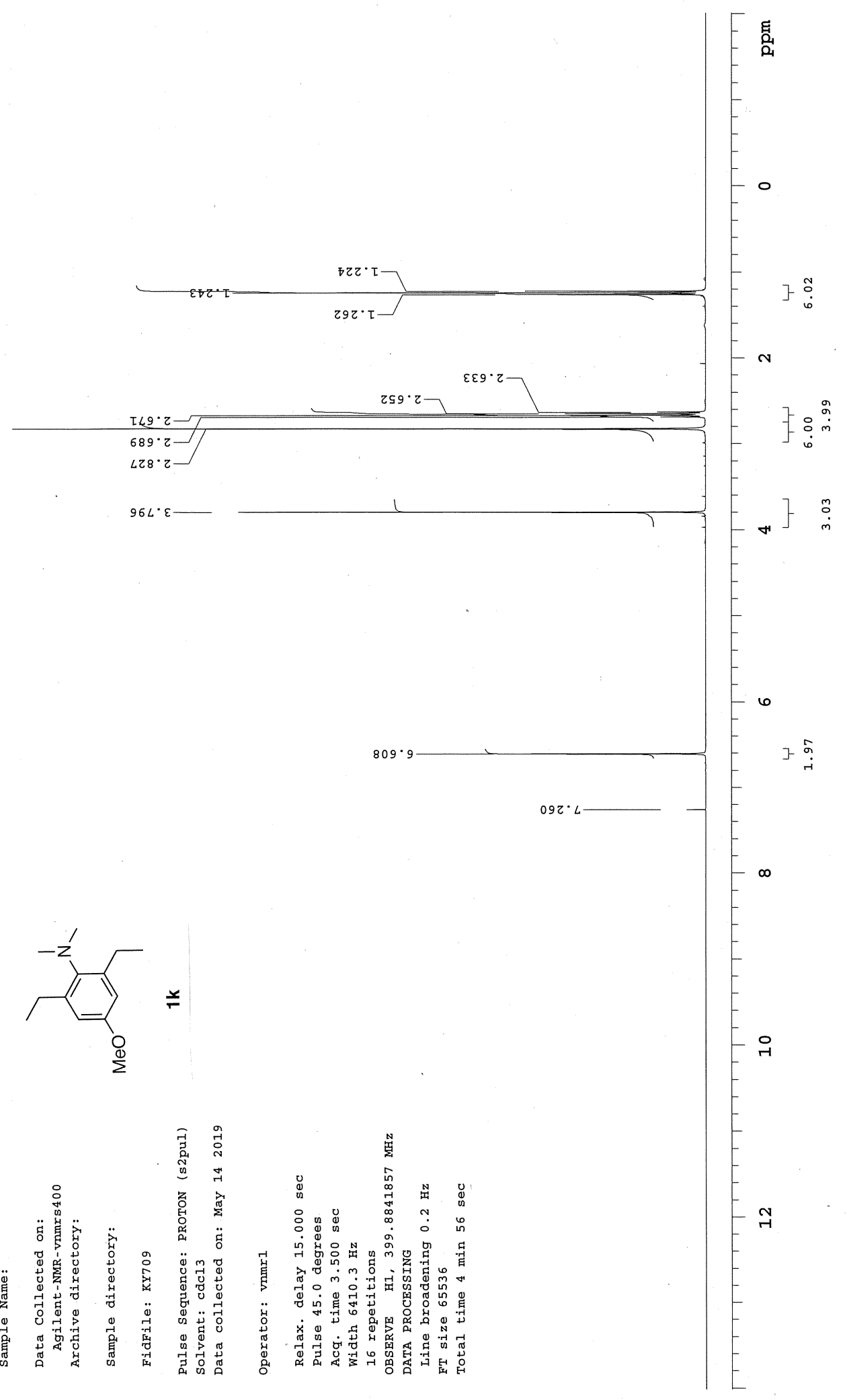

Figure S32. ${ }^{1} \mathrm{H}$ NMR Spectrum of $\mathbf{1 k}\left(400 \mathrm{MHz}, \mathrm{CDCl}_{3}\right)$ 


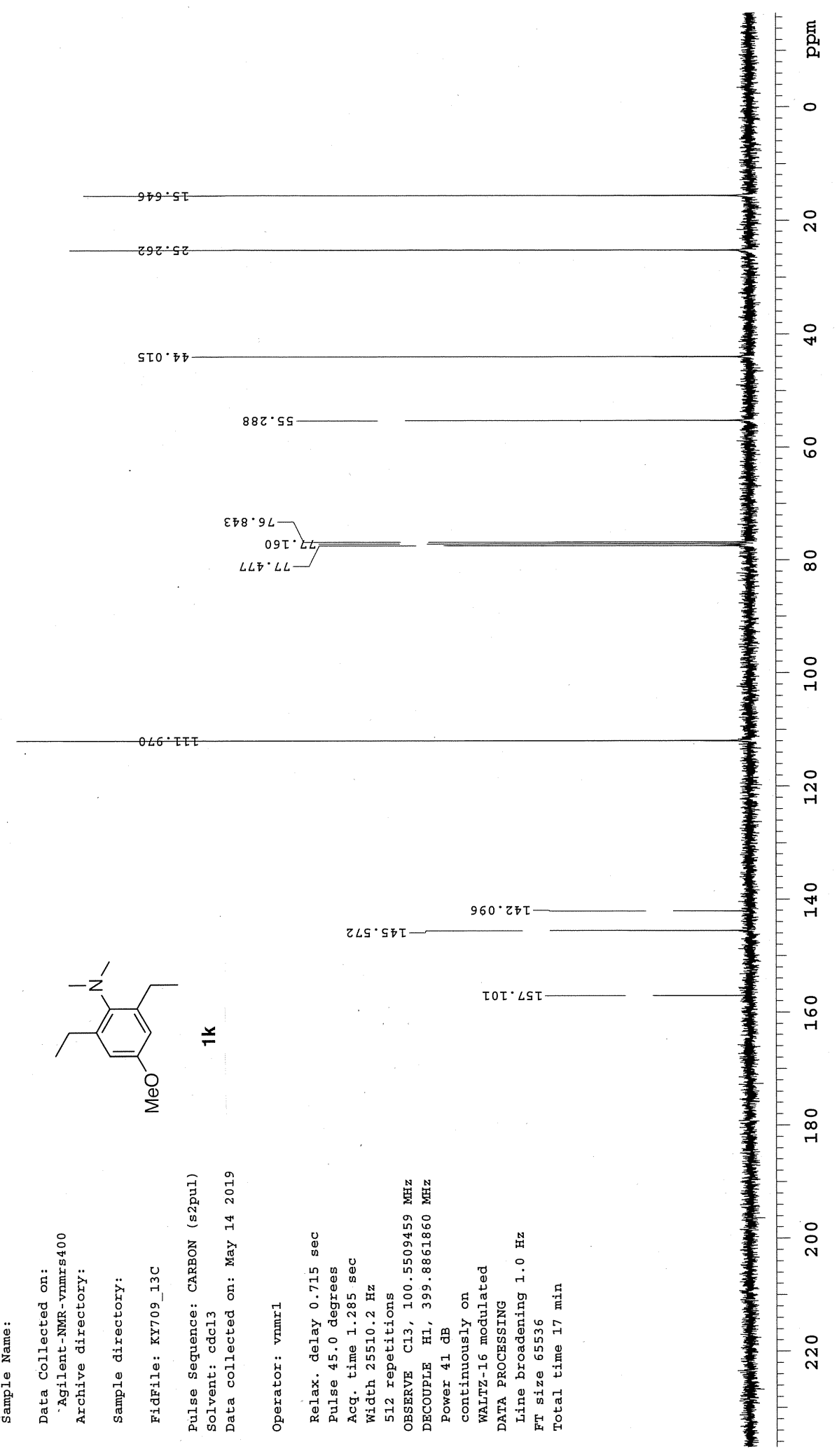

Figure S33. ${ }^{13} \mathrm{C}$ NMR Spectrum of $\mathbf{1 k}\left(101 \mathrm{MHz}, \mathrm{CDCl}_{3}\right)$ 


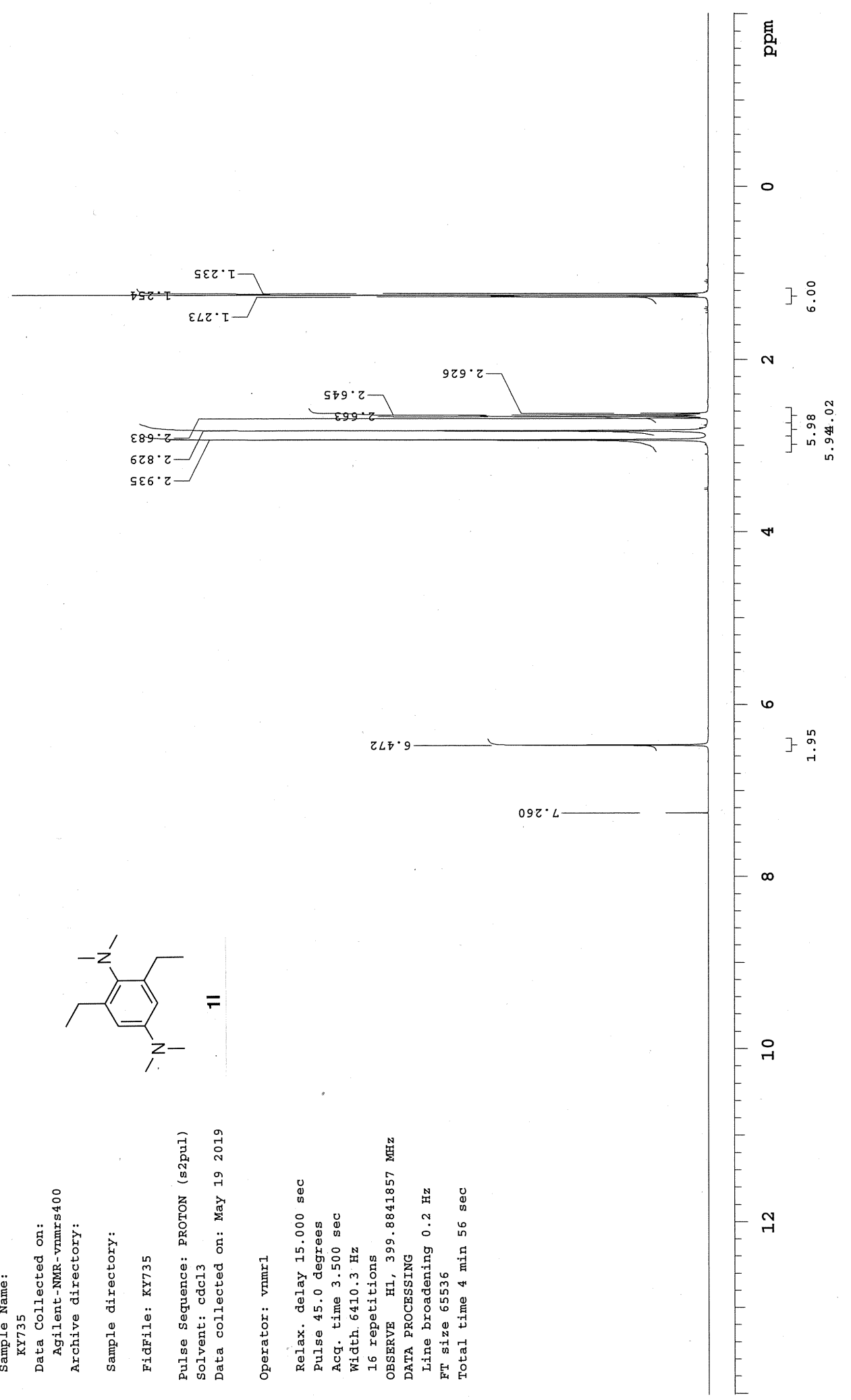

Figure S34. ${ }^{1} \mathrm{H}$ NMR Spectrum of $11\left(400 \mathrm{MHz}, \mathrm{CDCl}_{3}\right)$ 


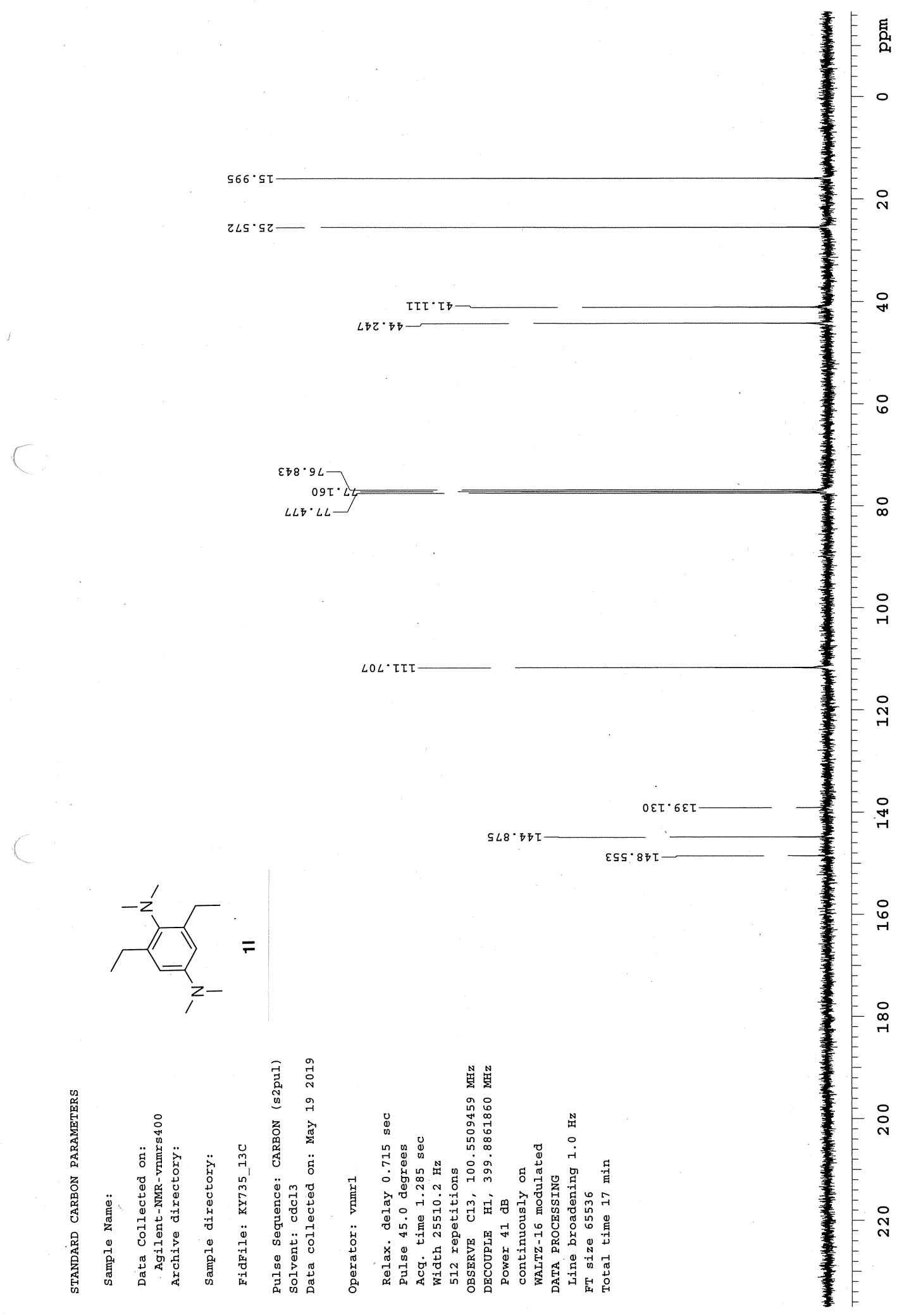

Figure S35. ${ }^{13} \mathrm{C}$ NMR Spectrum of $11\left(101 \mathrm{MHz}, \mathrm{CDCl}_{3}\right)$

S89 


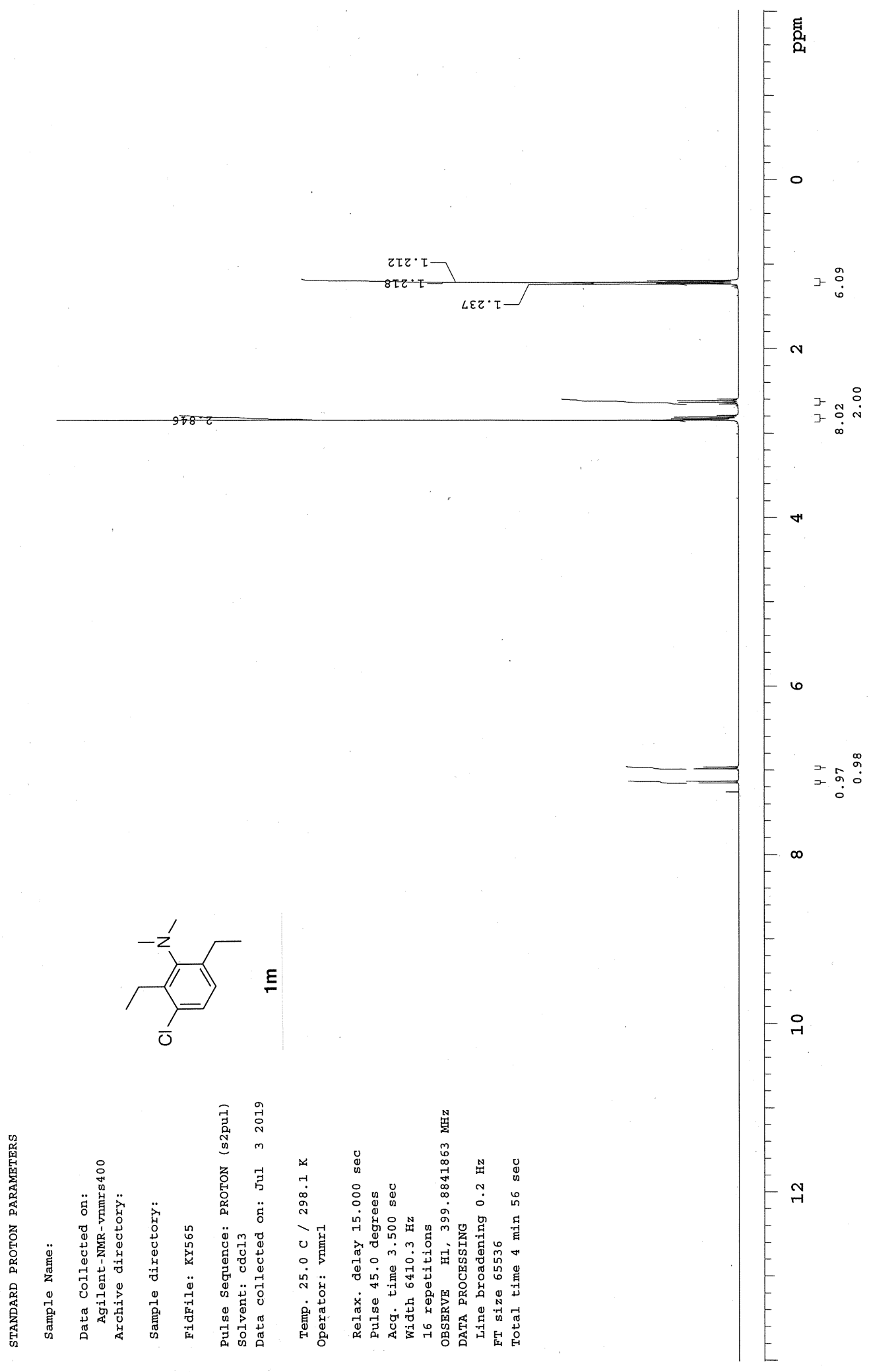

Figure S36. ${ }^{1} \mathrm{H}$ NMR Spectrum of $1 \mathbf{m}\left(400 \mathrm{MHz}, \mathrm{CDCl}_{3}\right)$ 


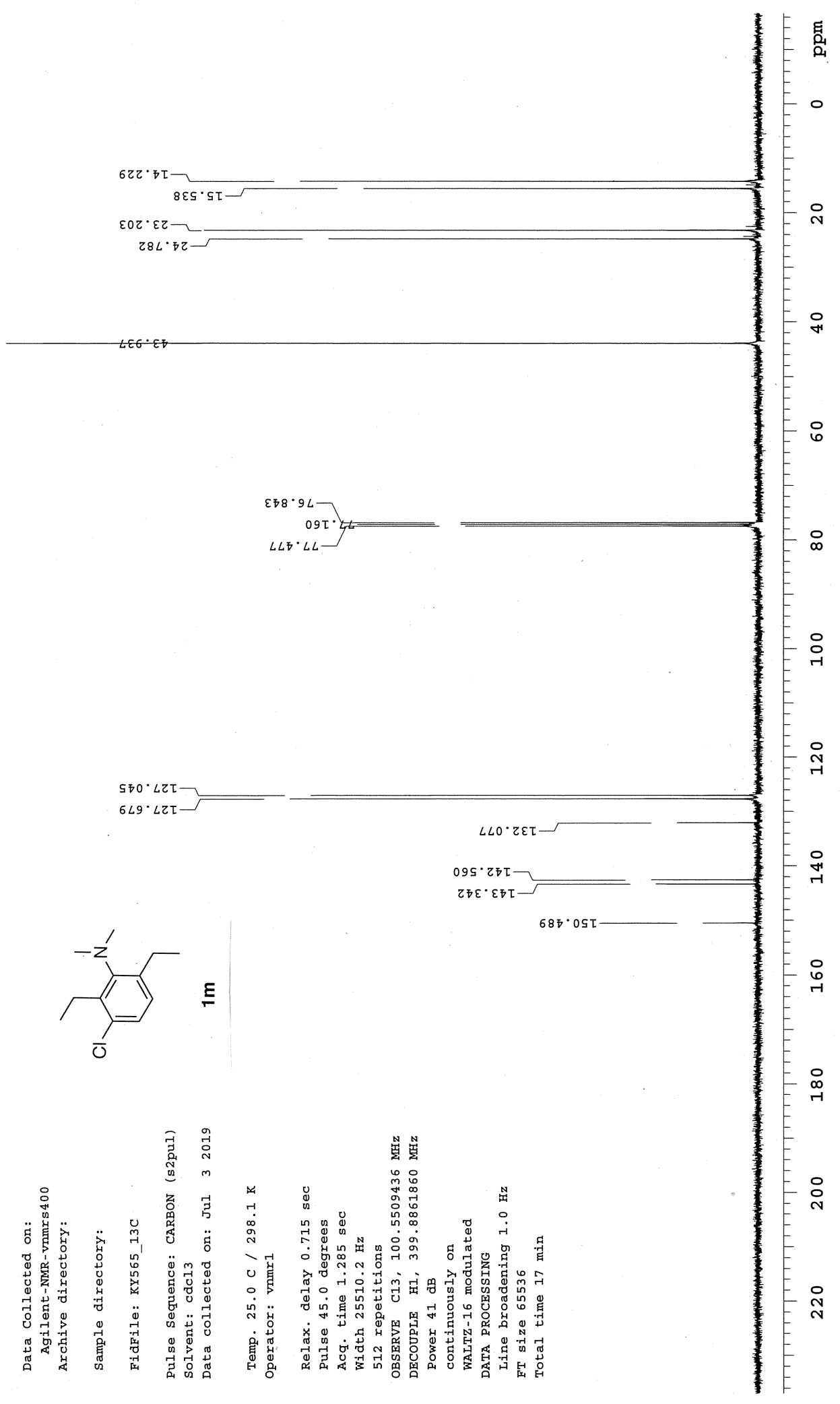

Figure S37. ${ }^{13} \mathrm{C}$ NMR Spectrum of $\mathbf{1 m}\left(101 \mathrm{MHz}, \mathrm{CDCl}_{3}\right)$ 


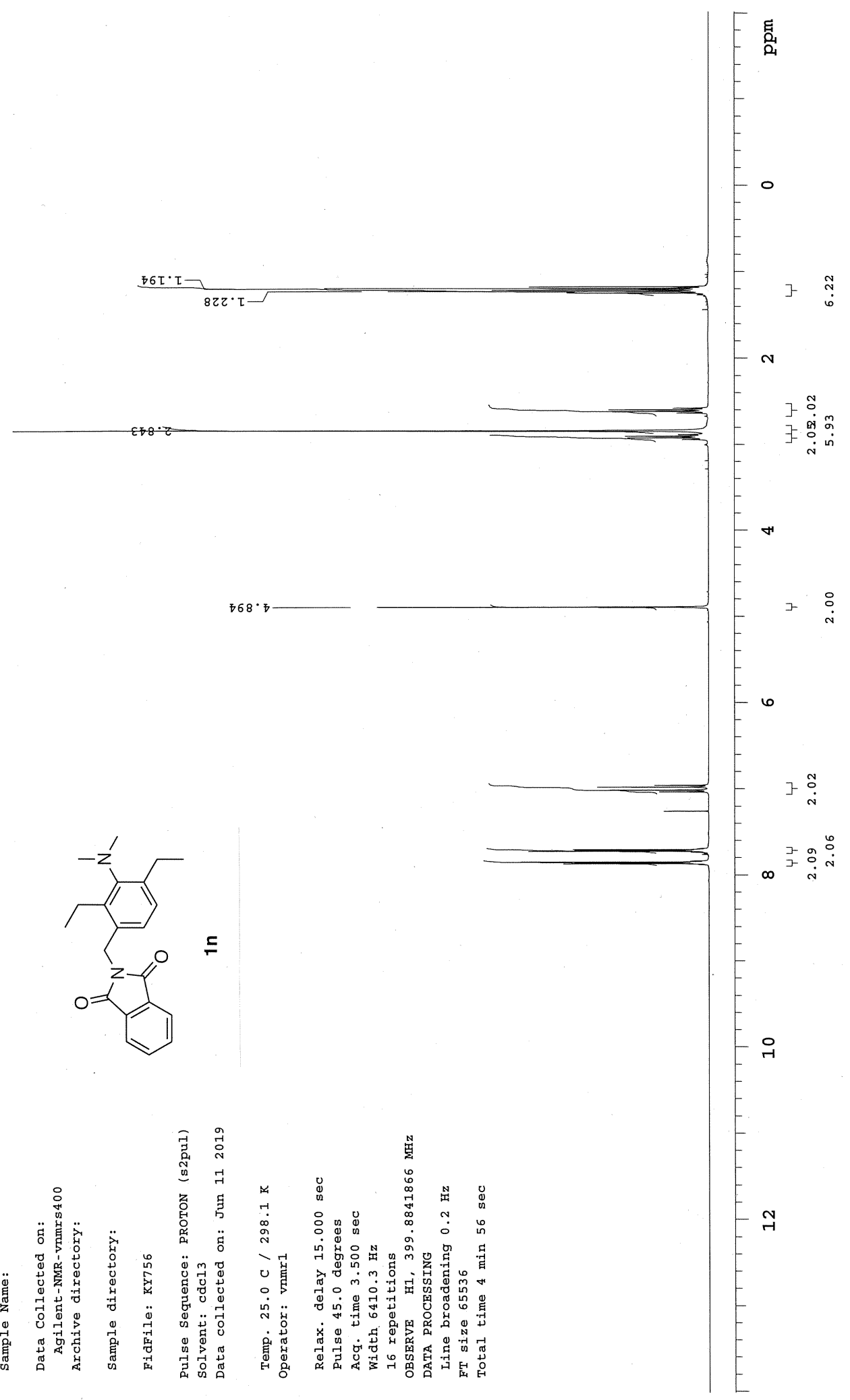

Figure S38. ${ }^{1} \mathrm{H}$ NMR Spectrum of 1 n $\left(400 \mathrm{MHz}, \mathrm{CDCl}_{3}\right)$ 


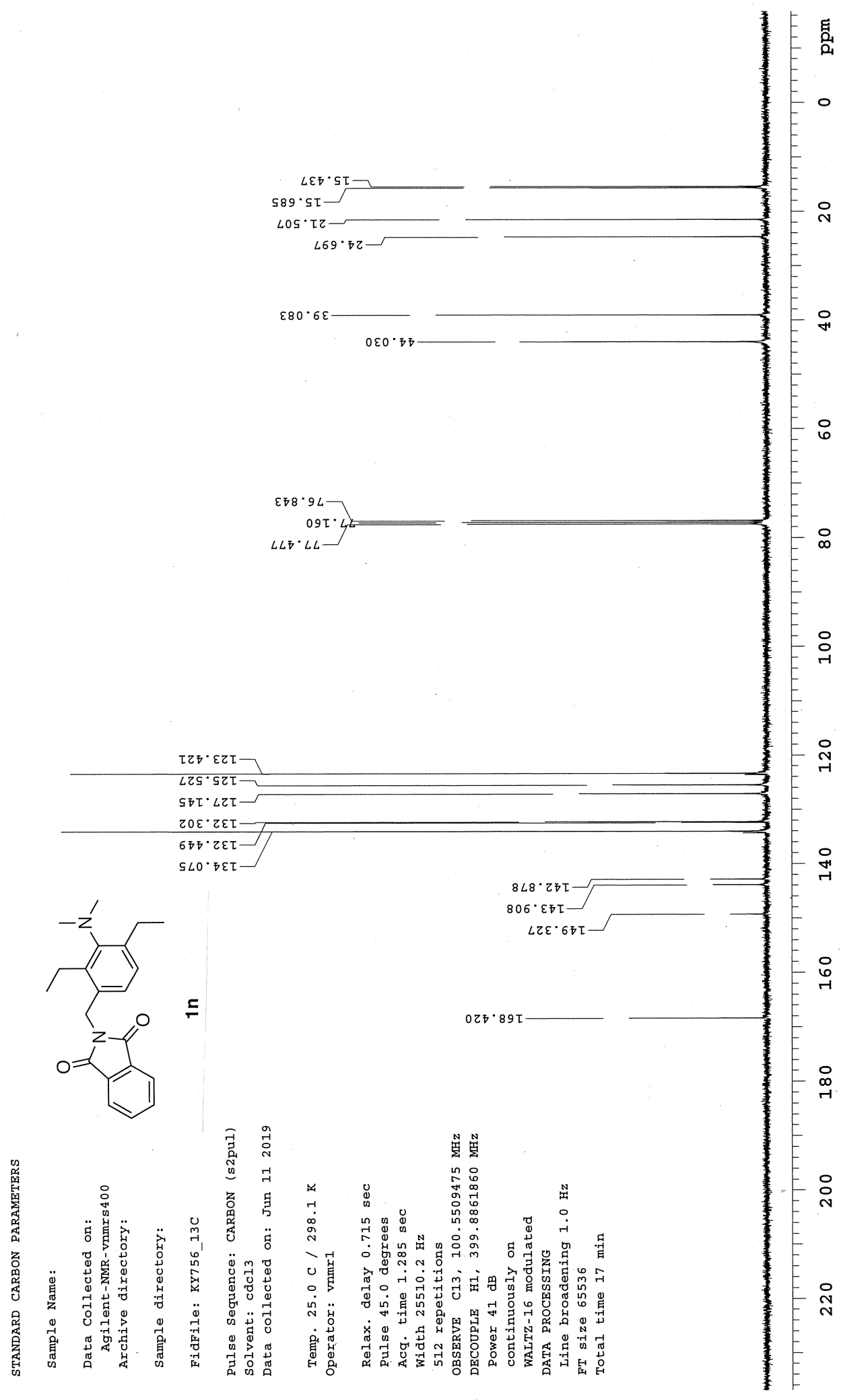

Figure S39. ${ }^{13} \mathrm{C}$ NMR Spectrum of 1 n $\left(101 \mathrm{MHz}, \mathrm{CDCl}_{3}\right)$ 


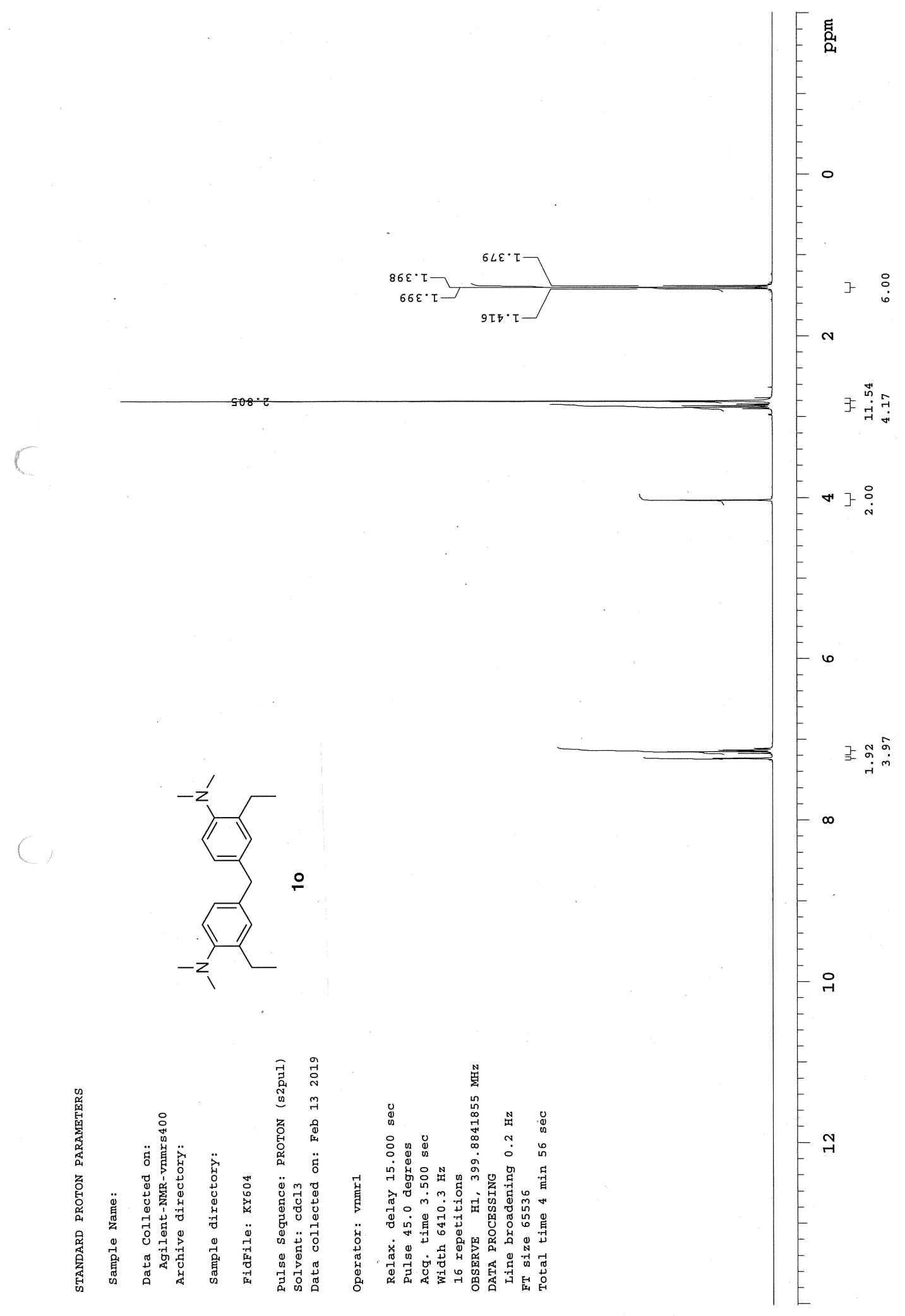

Figure S40. ${ }^{1} \mathrm{H}$ NMR Spectrum of $10\left(400 \mathrm{MHz}, \mathrm{CDCl}_{3}\right)$ 


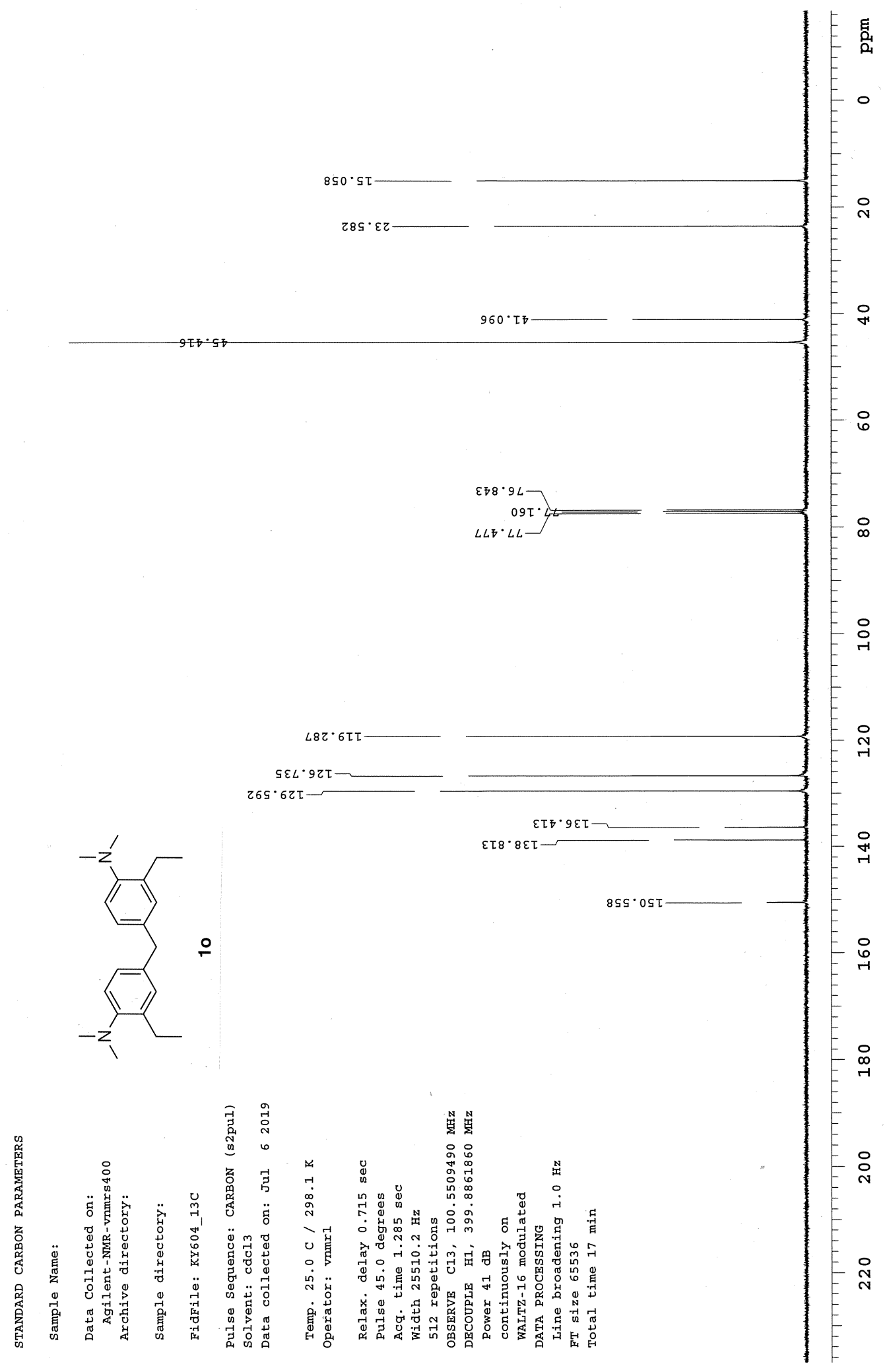

Figure S41. ${ }^{13} \mathrm{C}$ NMR Spectrum of $10\left(101 \mathrm{MHz}, \mathrm{CDCl}_{3}\right)$ 


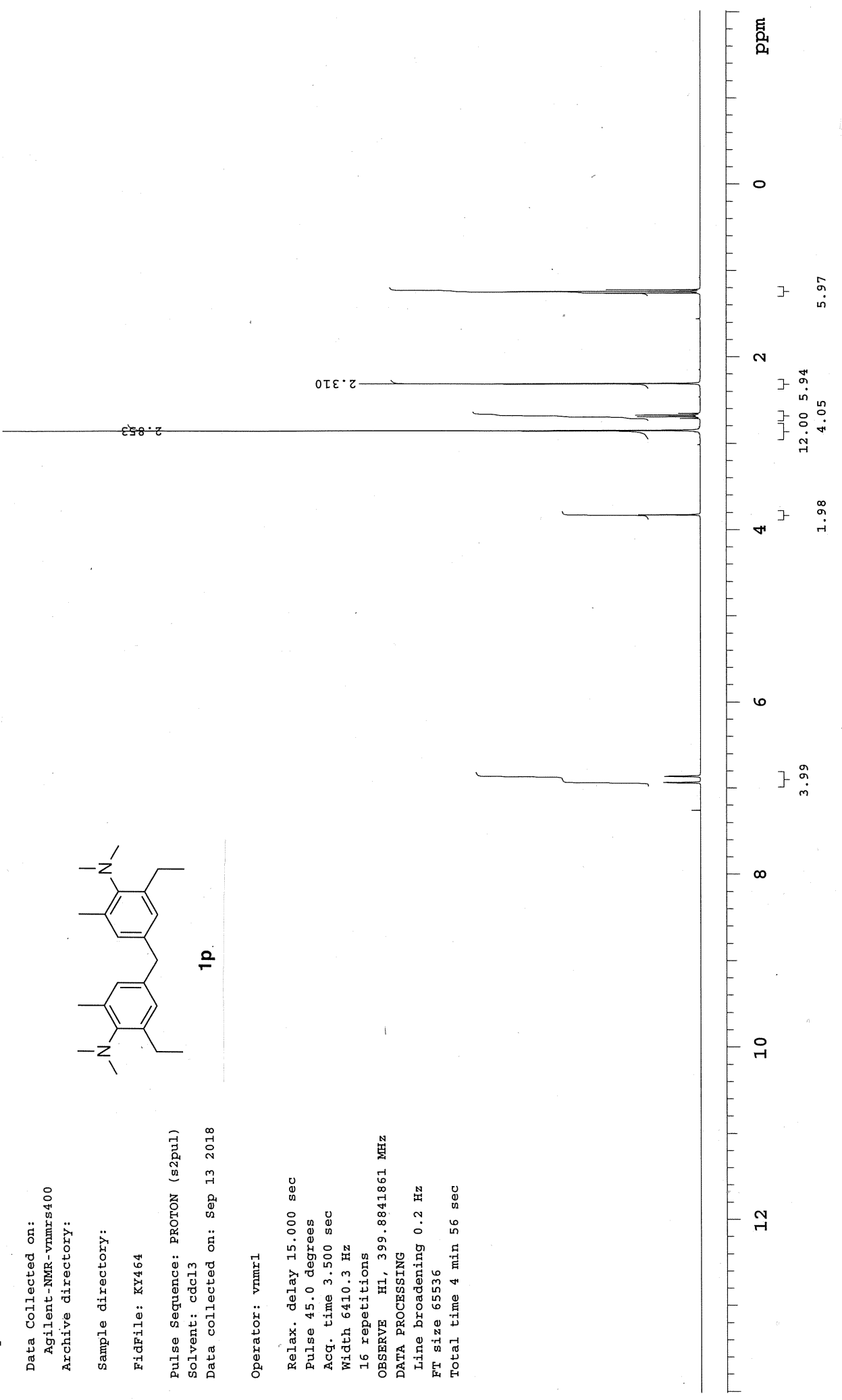

Figure S42. ${ }^{1} \mathrm{H}$ NMR Spectrum of $1 p\left(400 \mathrm{MHz}, \mathrm{CDCl}_{3}\right)$ 


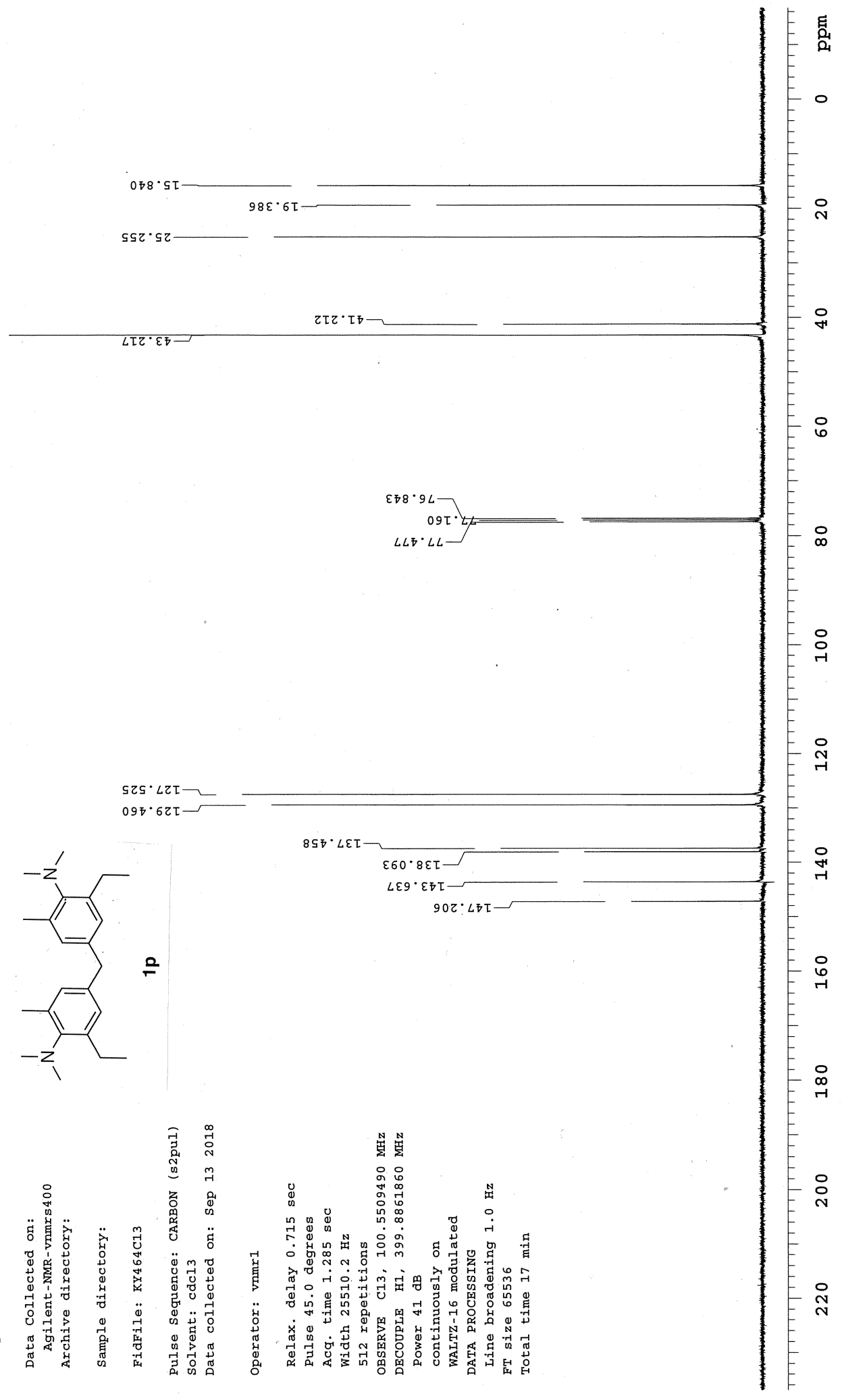

Figure S43. ${ }^{13} \mathrm{C}$ NMR Spectrum of $\mathbf{1 p}\left(101 \mathrm{MHz}, \mathrm{CDCl}_{3}\right)$ 


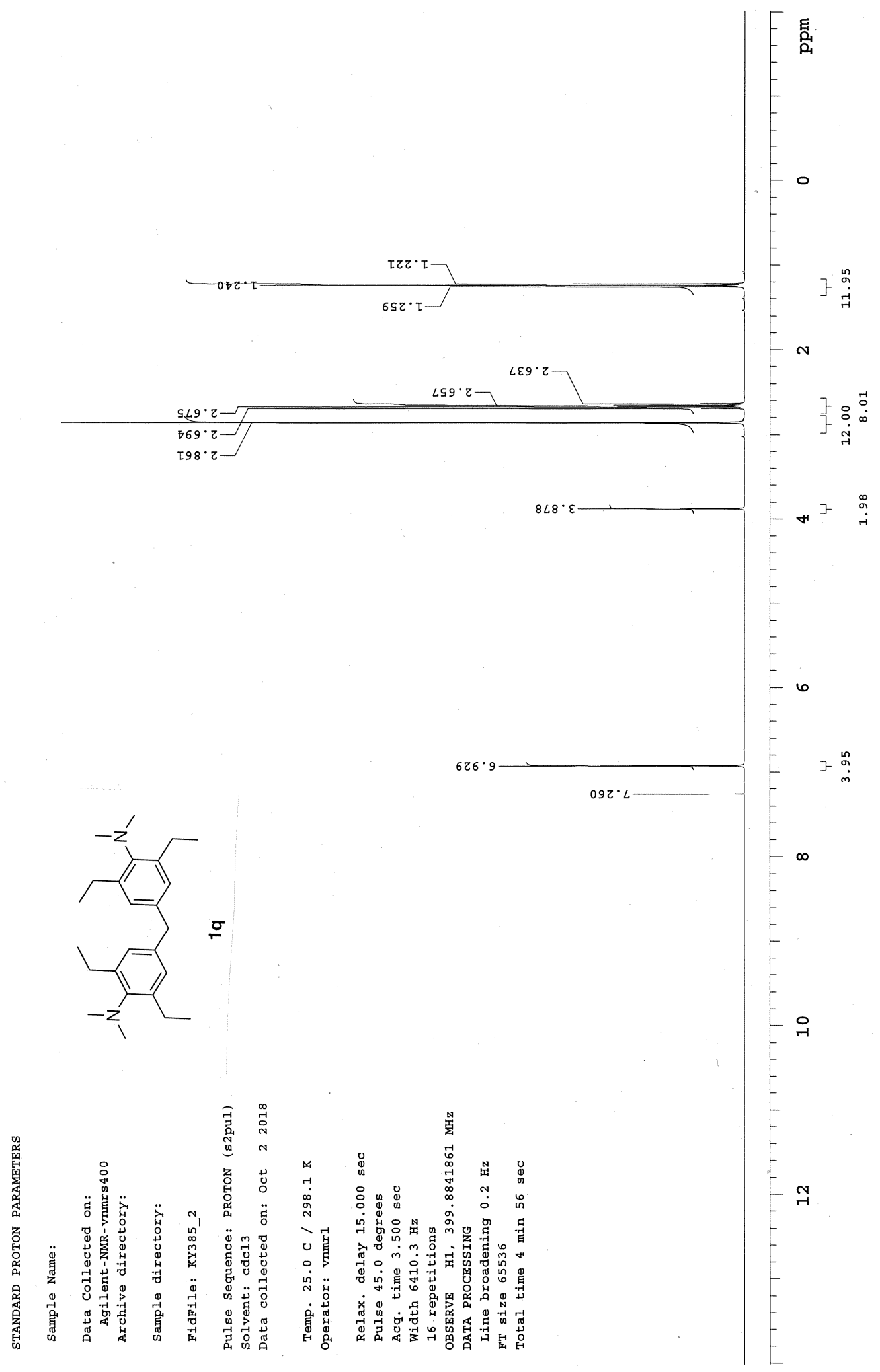

Figure S44. ${ }^{1} \mathrm{H}$ NMR Spectrum of $1 q\left(400 \mathrm{MHz}, \mathrm{CDCl}_{3}\right)$ 


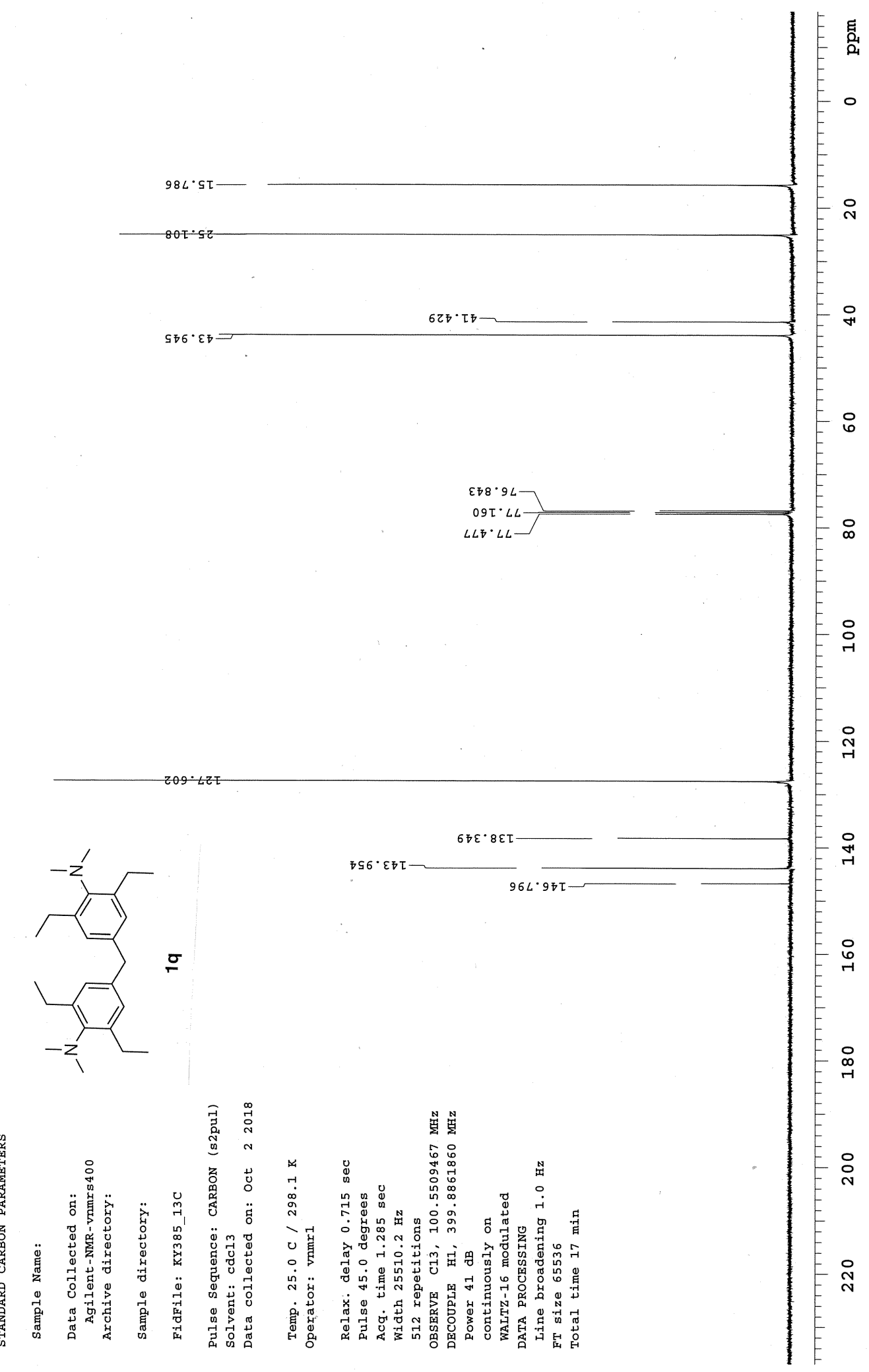

Figure S45. ${ }^{13} \mathrm{C}$ NMR Spectrum of $1 q\left(101 \mathrm{MHz}, \mathrm{CDCl}_{3}\right)$ 


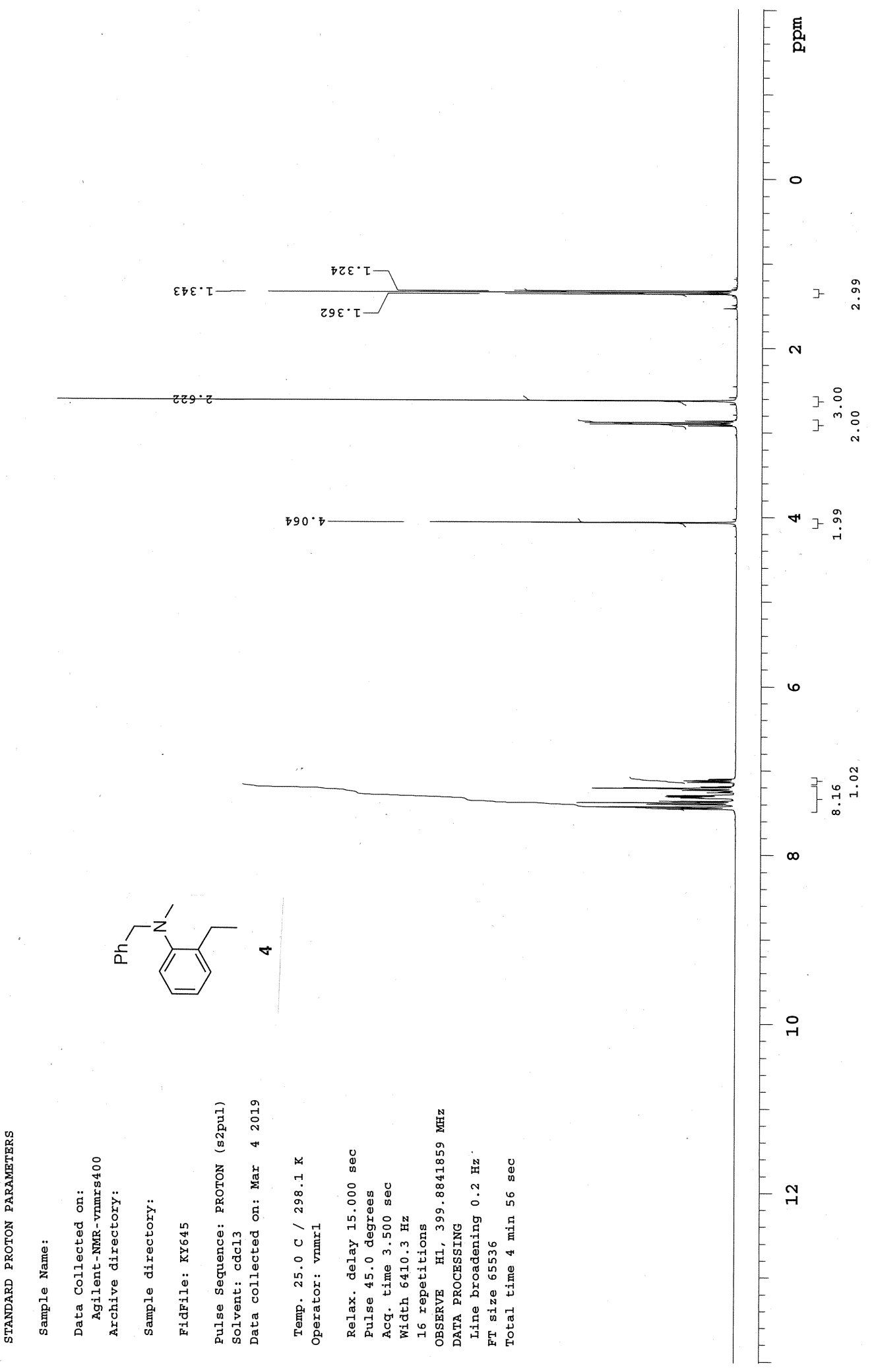

Figure S46. ${ }^{1} \mathrm{H}$ NMR Spectrum of $4\left(400 \mathrm{MHz}, \mathrm{CDCl}_{3}\right)$ 


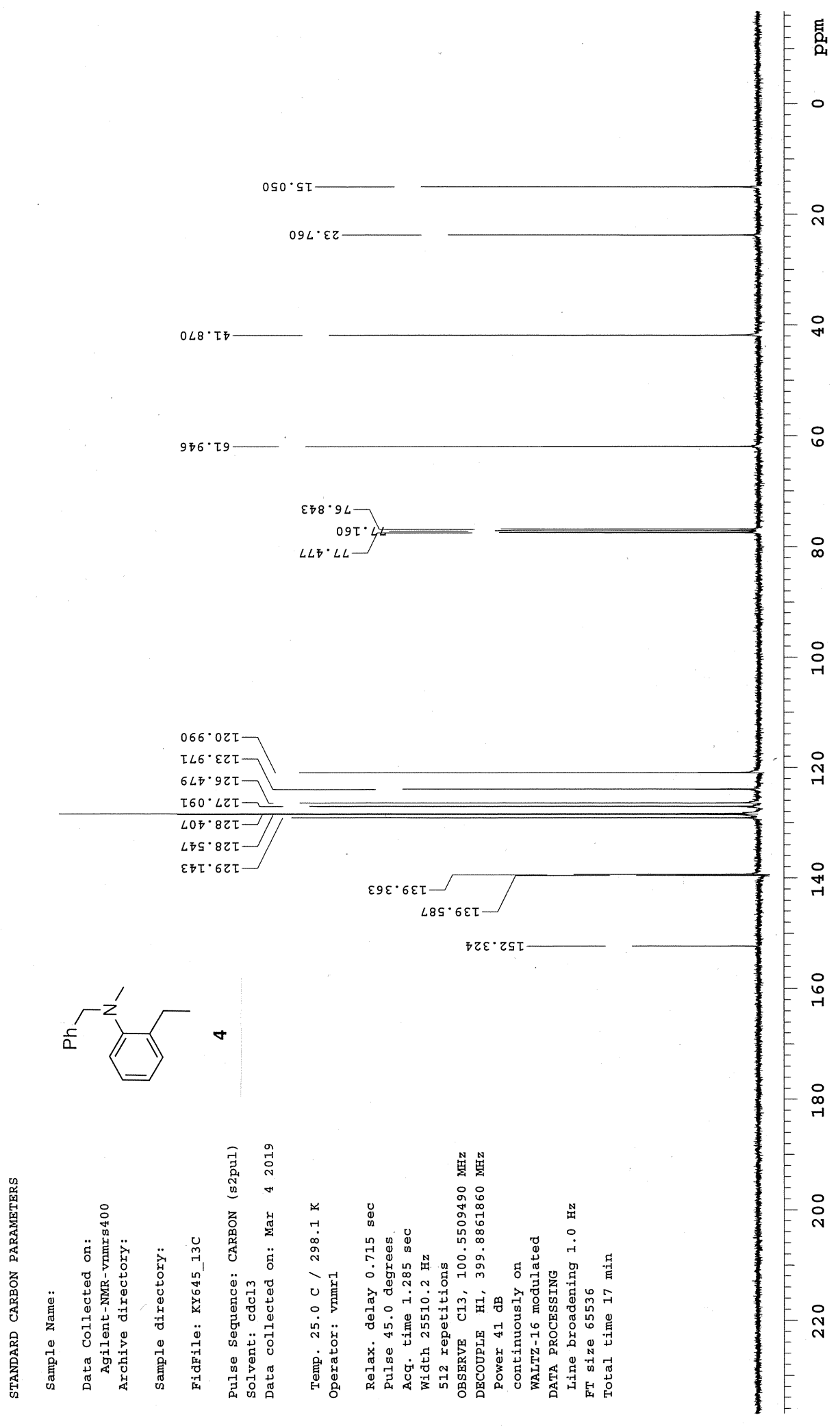

Figure S47. ${ }^{13} \mathrm{C}$ NMR Spectrum of $4\left(101 \mathrm{MHz}, \mathrm{CDCl}_{3}\right)$ 


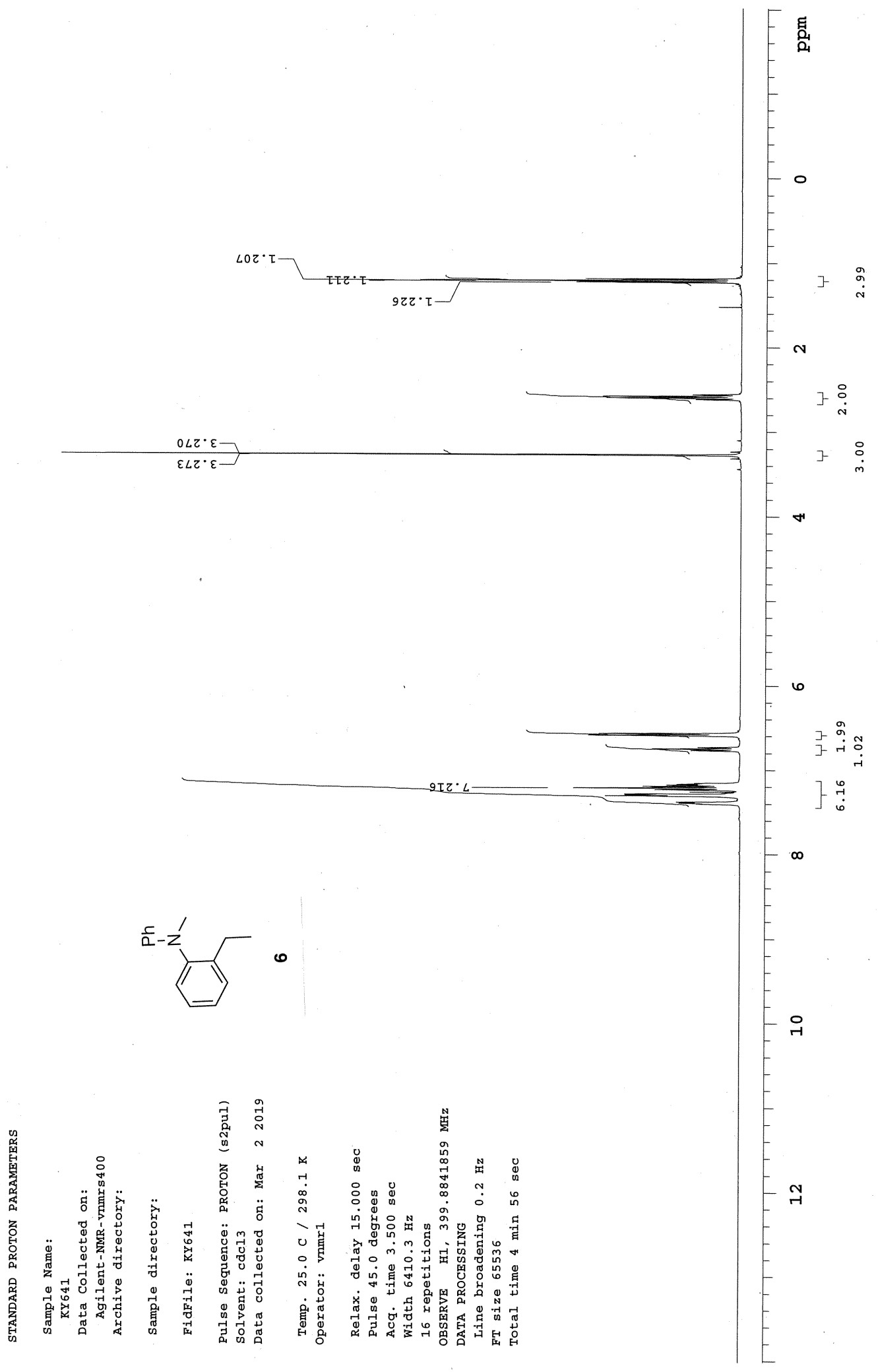

Figure S48. ${ }^{1} \mathrm{H}$ NMR Spectrum of $6\left(400 \mathrm{MHz}, \mathrm{CDCl}_{3}\right)$ 


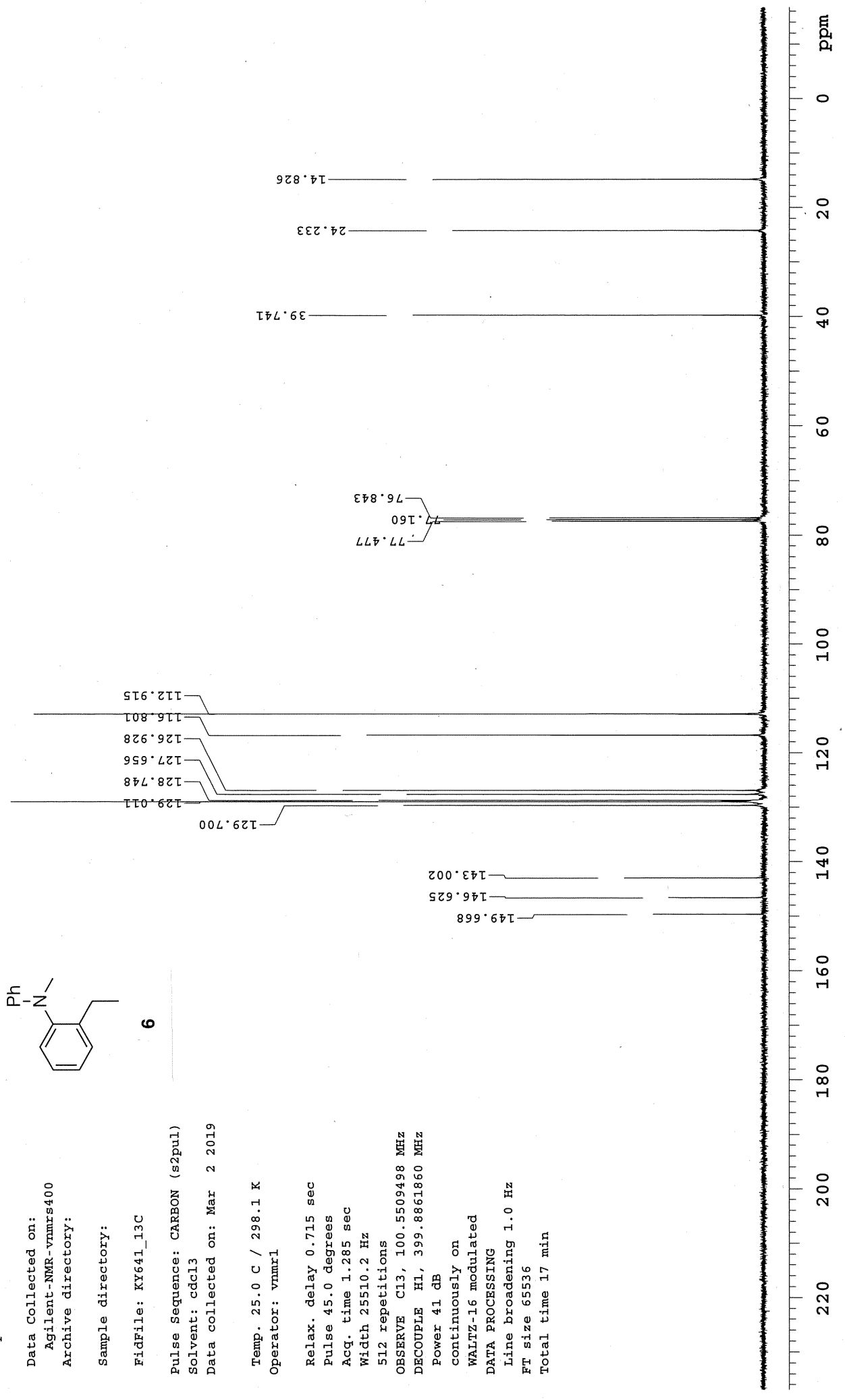

Figure S49. ${ }^{13} \mathrm{C}$ NMR Spectrum of $6\left(101 \mathrm{MHz}, \mathrm{CDCl}_{3}\right)$ 


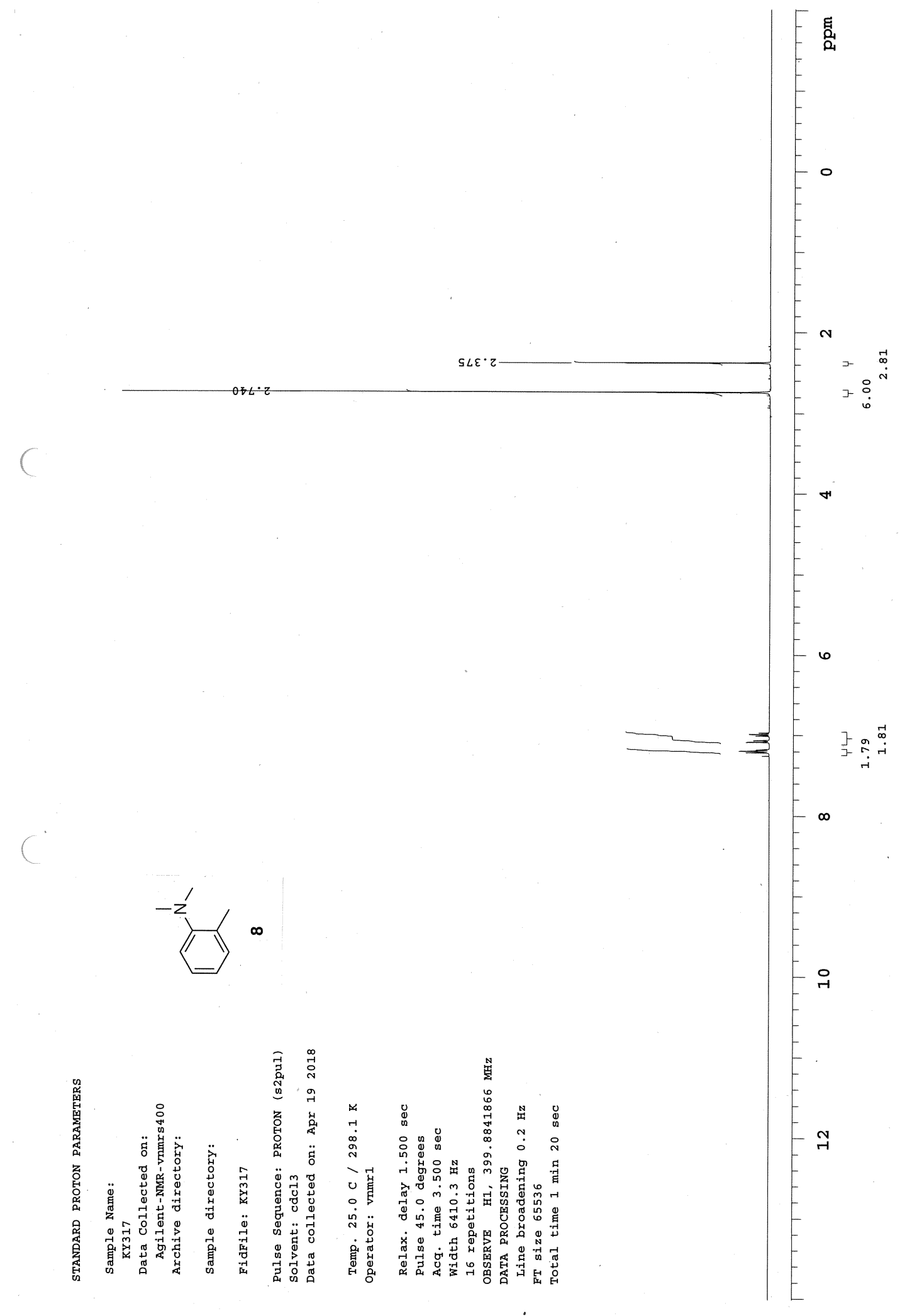

Figure S50. ${ }^{1} \mathrm{H}$ NMR Spectrum of $8\left(400 \mathrm{MHz}, \mathrm{CDCl}_{3}\right)$ 


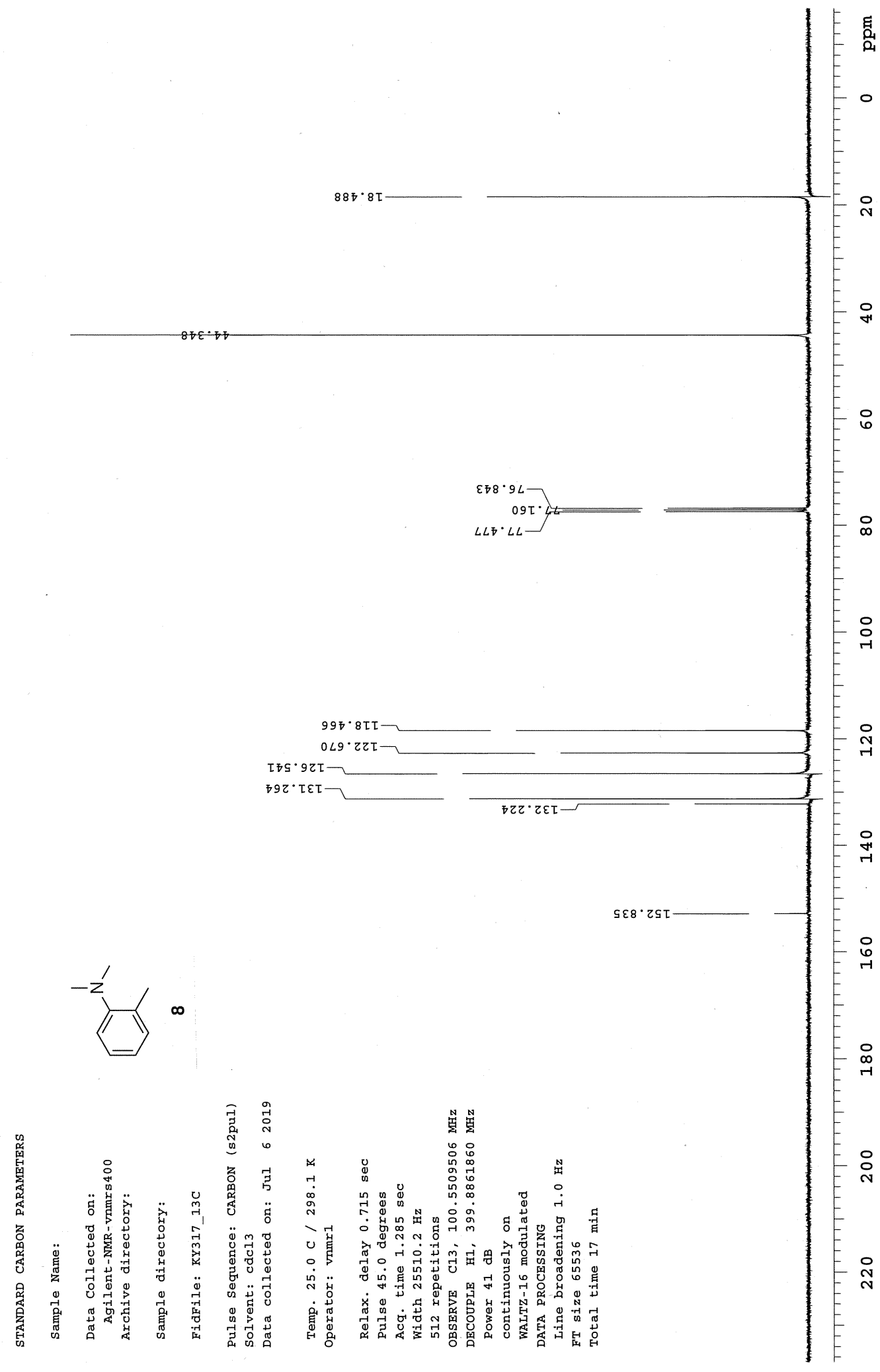

Figure S51. ${ }^{13} \mathrm{C}$ NMR Spectrum of $8\left(101 \mathrm{MHz}, \mathrm{CDCl}_{3}\right)$ 


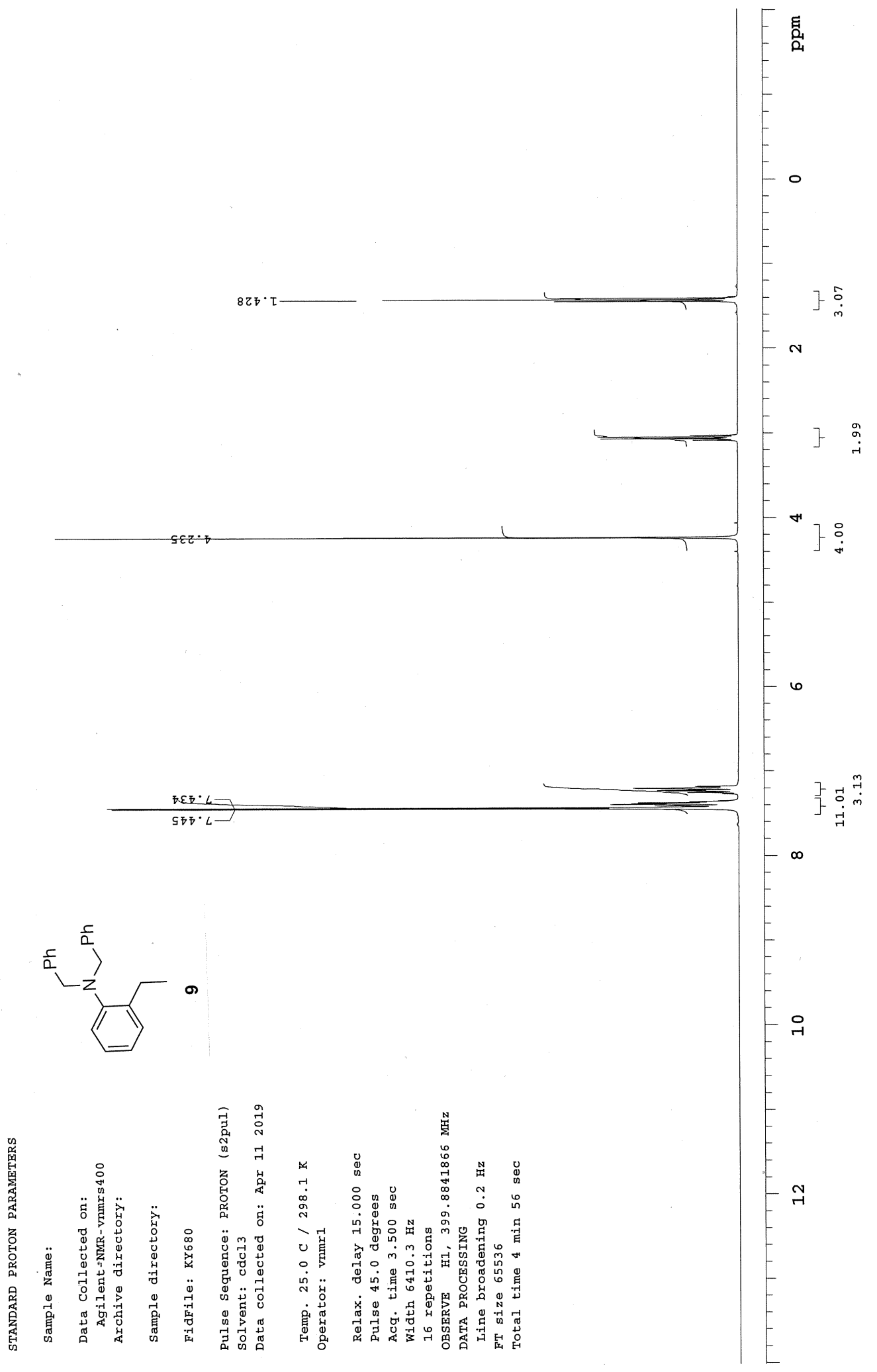

Figure S52. ${ }^{1} \mathrm{H}$ NMR Spectrum of $9\left(400 \mathrm{MHz}, \mathrm{CDCl}_{3}\right)$ 


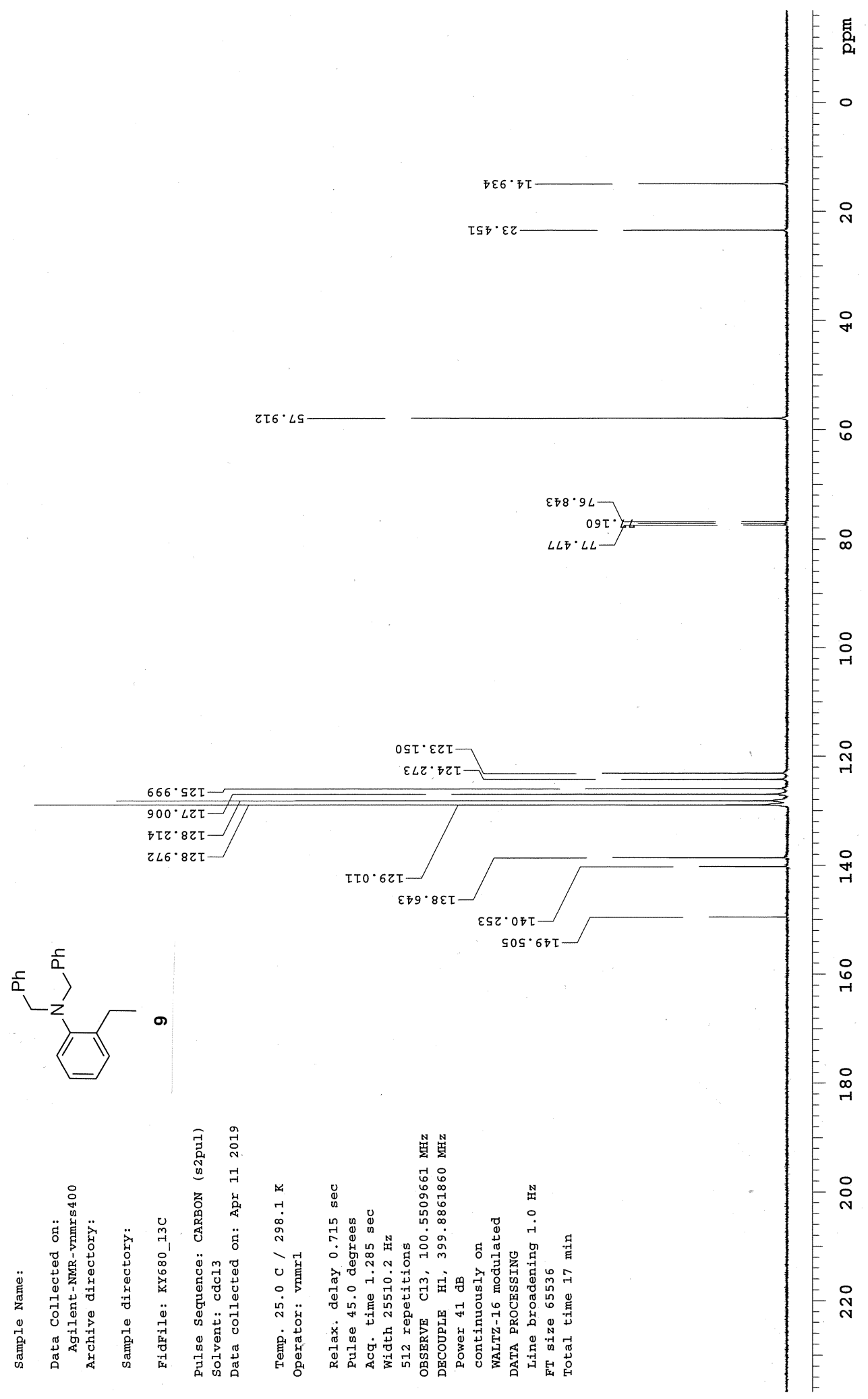

Figure S53. ${ }^{13} \mathrm{C}$ NMR Spectrum of $9\left(101 \mathrm{MHz}, \mathrm{CDCl}_{3}\right)$ 


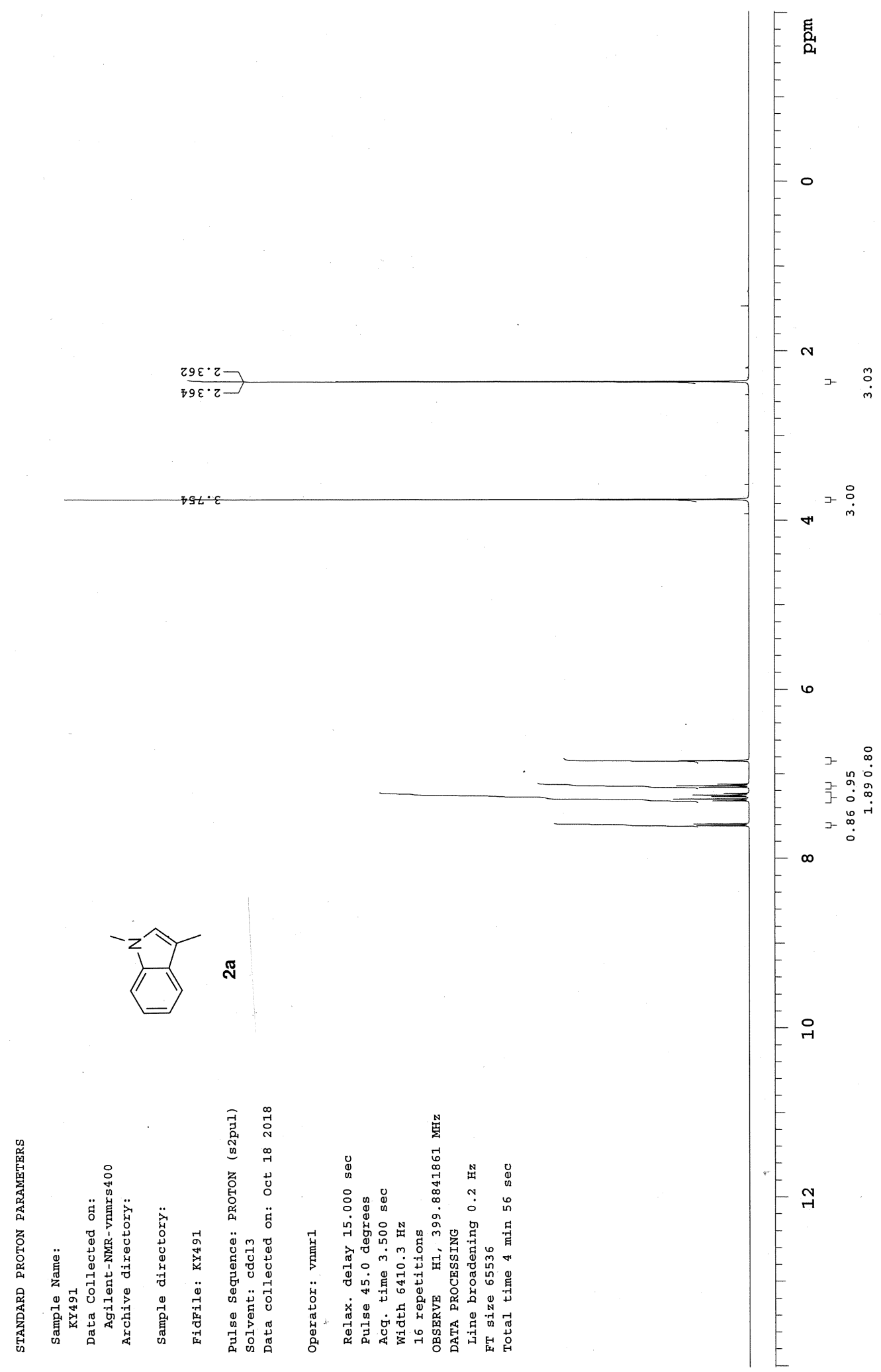

Figure S54. ${ }^{1} \mathrm{H}$ NMR Spectrum of $\mathbf{2 a}\left(400 \mathrm{MHz}, \mathrm{CDCl}_{3}\right)$ 


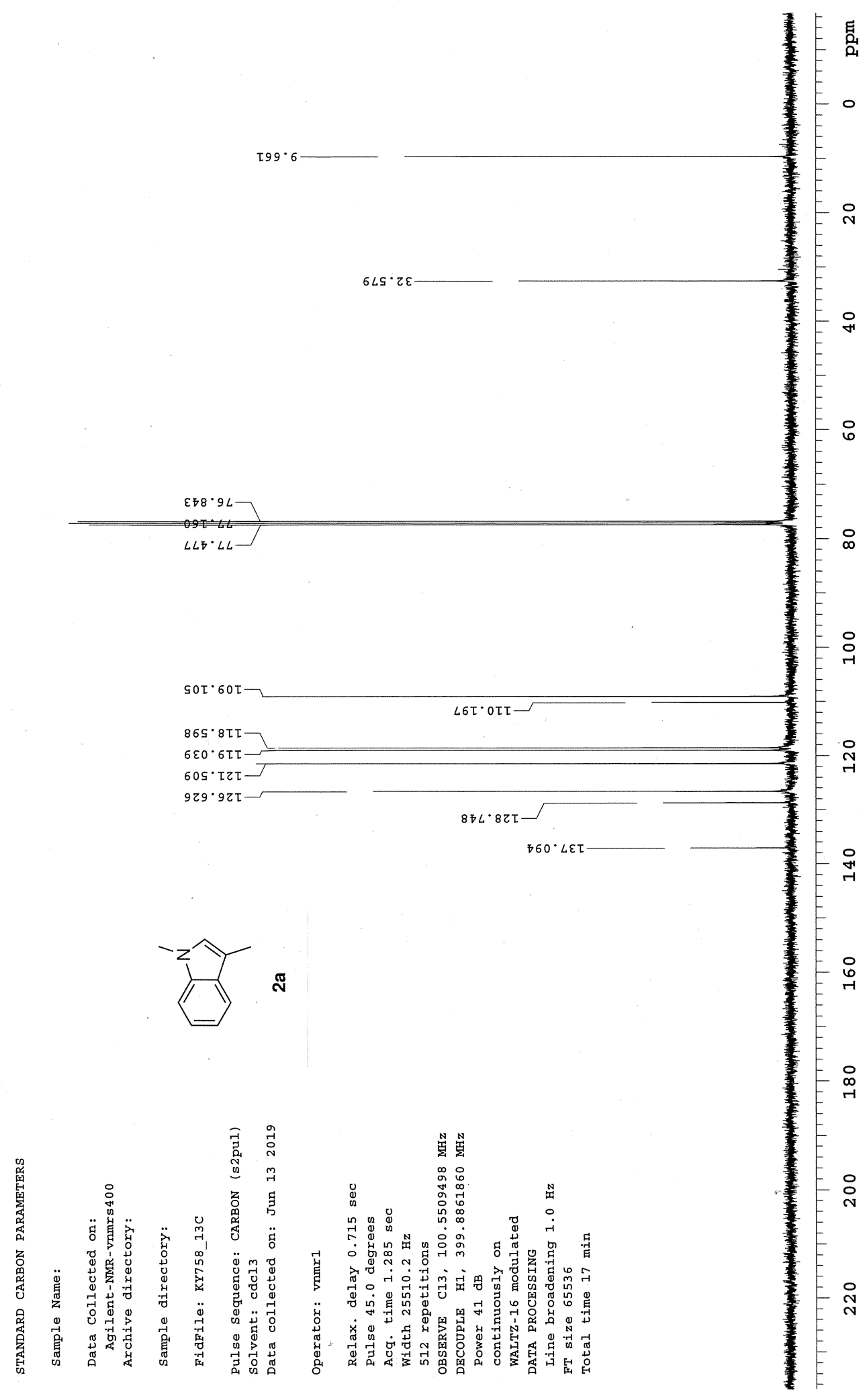

Figure S55. ${ }^{13} \mathrm{C}$ NMR Spectrum of $2 \mathbf{a}\left(101 \mathrm{MHz}, \mathrm{CDCl}_{3}\right)$ 


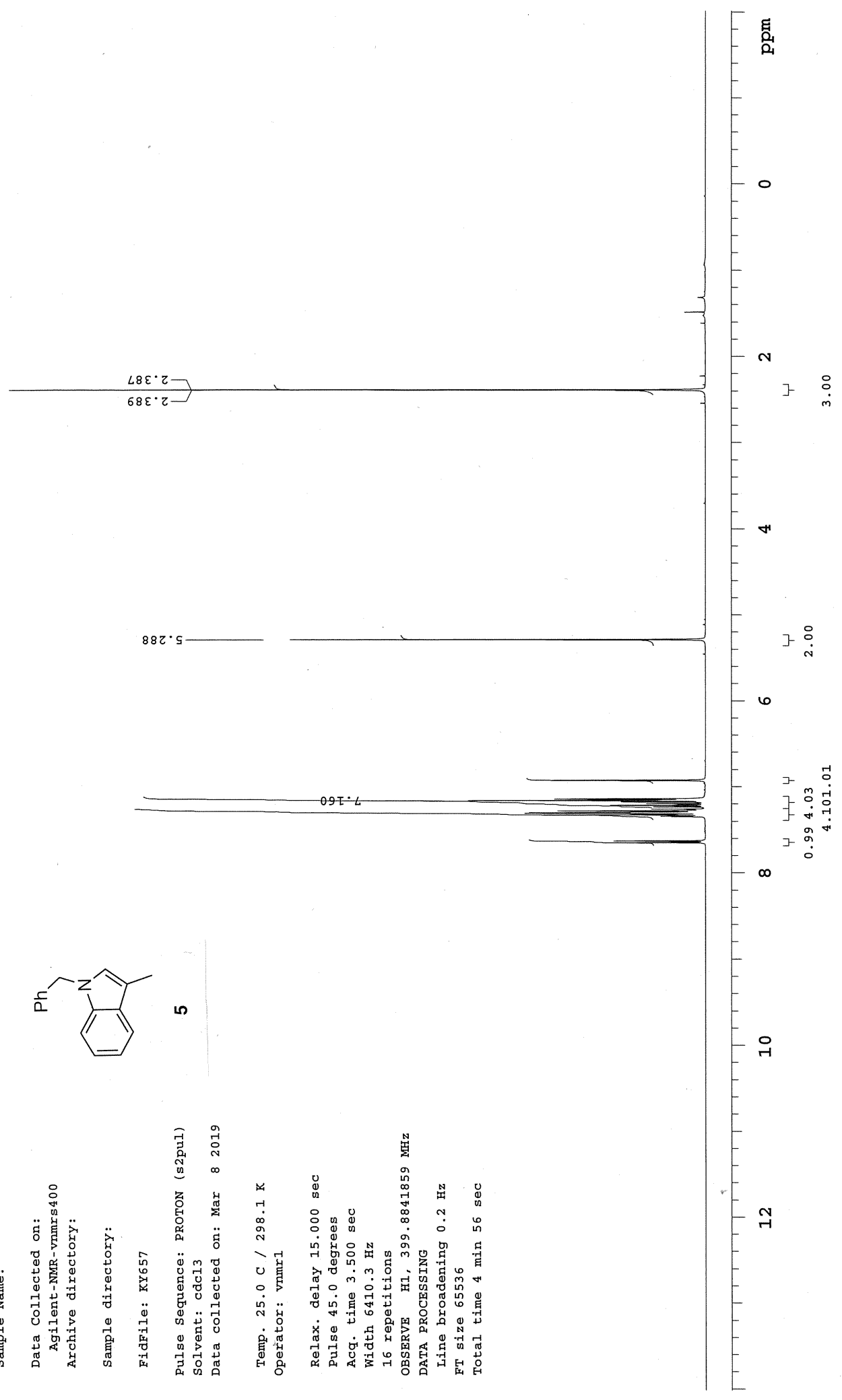

Figure S56. ${ }^{1} \mathrm{H}$ NMR Spectrum of $5\left(400 \mathrm{MHz}, \mathrm{CDCl}_{3}\right)$ 


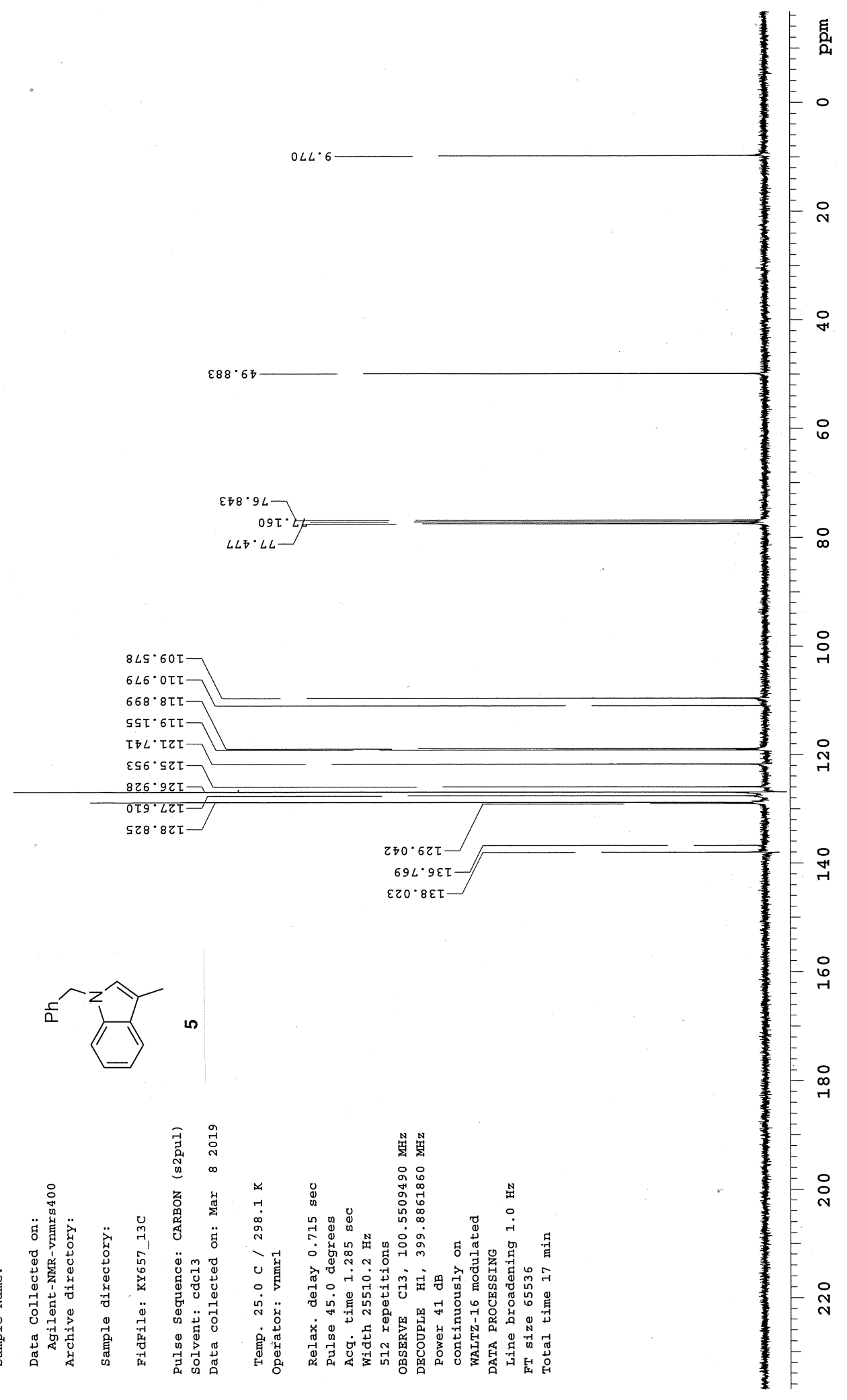

Figure S57. ${ }^{13} \mathrm{C}$ NMR Spectrum of $5\left(101 \mathrm{MHz}, \mathrm{CDCl}_{3}\right)$ 


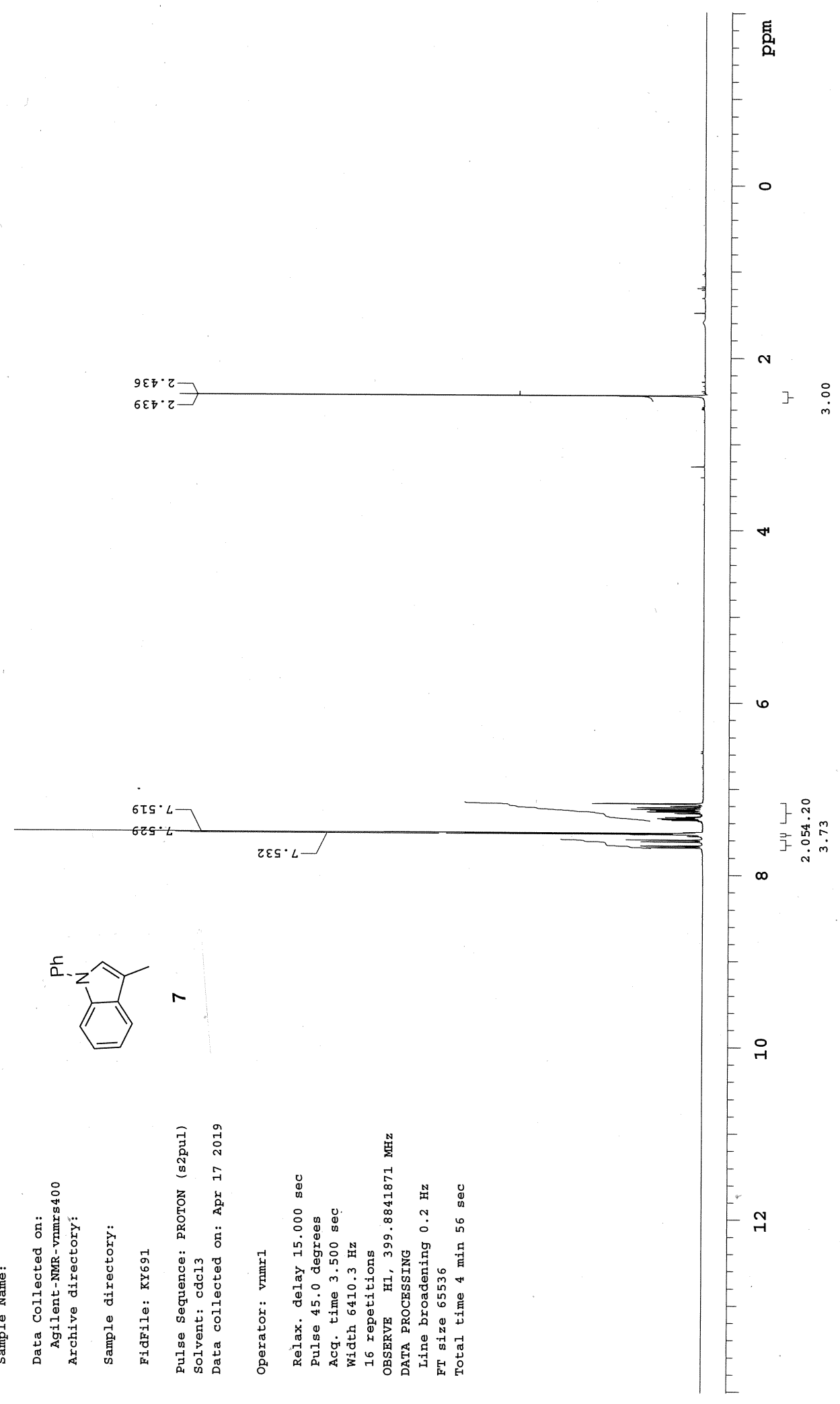

Figure S58. ${ }^{1} \mathrm{H}$ NMR Spectrum of $7\left(400 \mathrm{MHz}, \mathrm{CDCl}_{3}\right)$ 


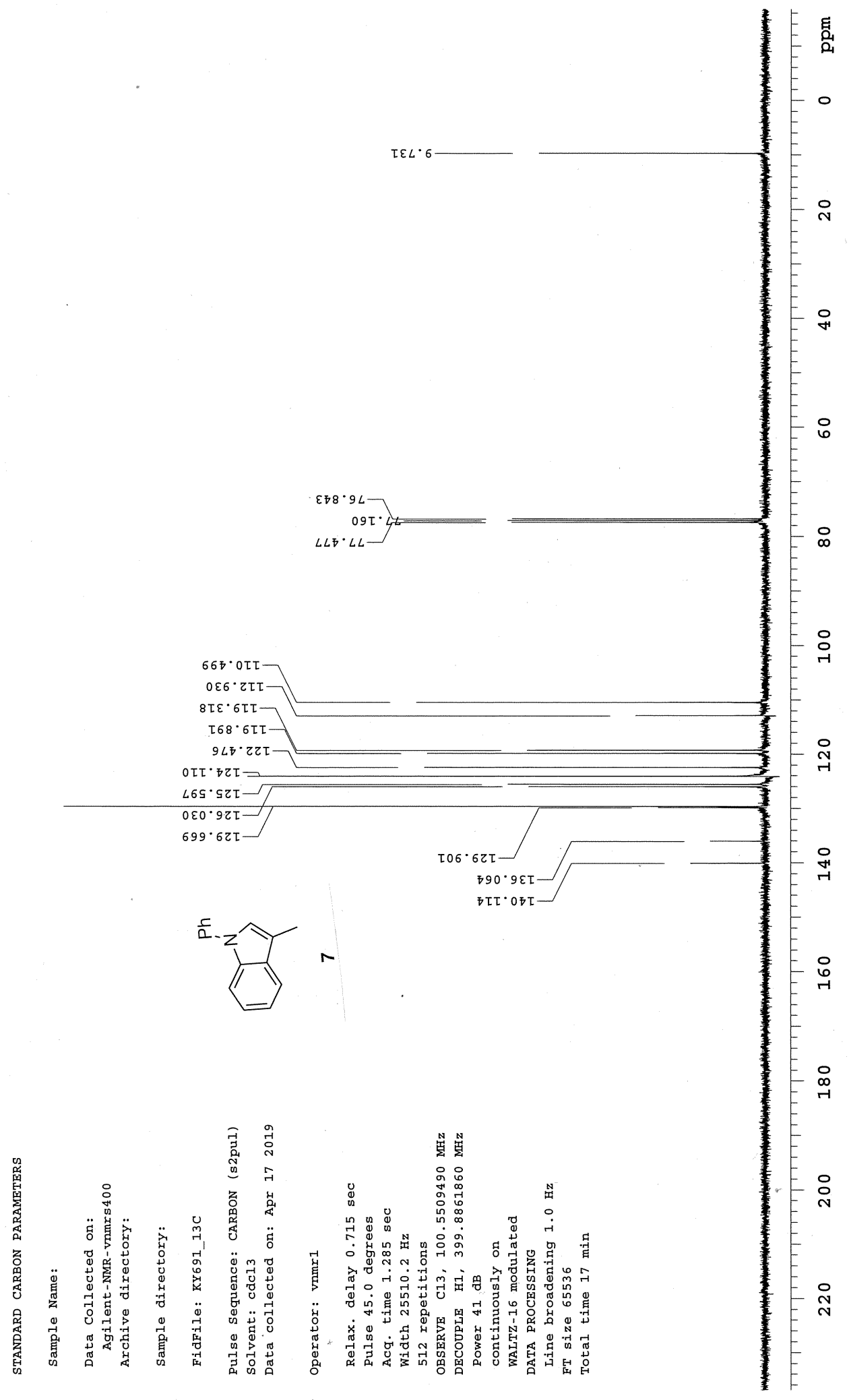

Figure S59. ${ }^{13} \mathrm{C}$ NMR Spectrum of $7\left(101 \mathrm{MHz}, \mathrm{CDCl}_{3}\right)$ 


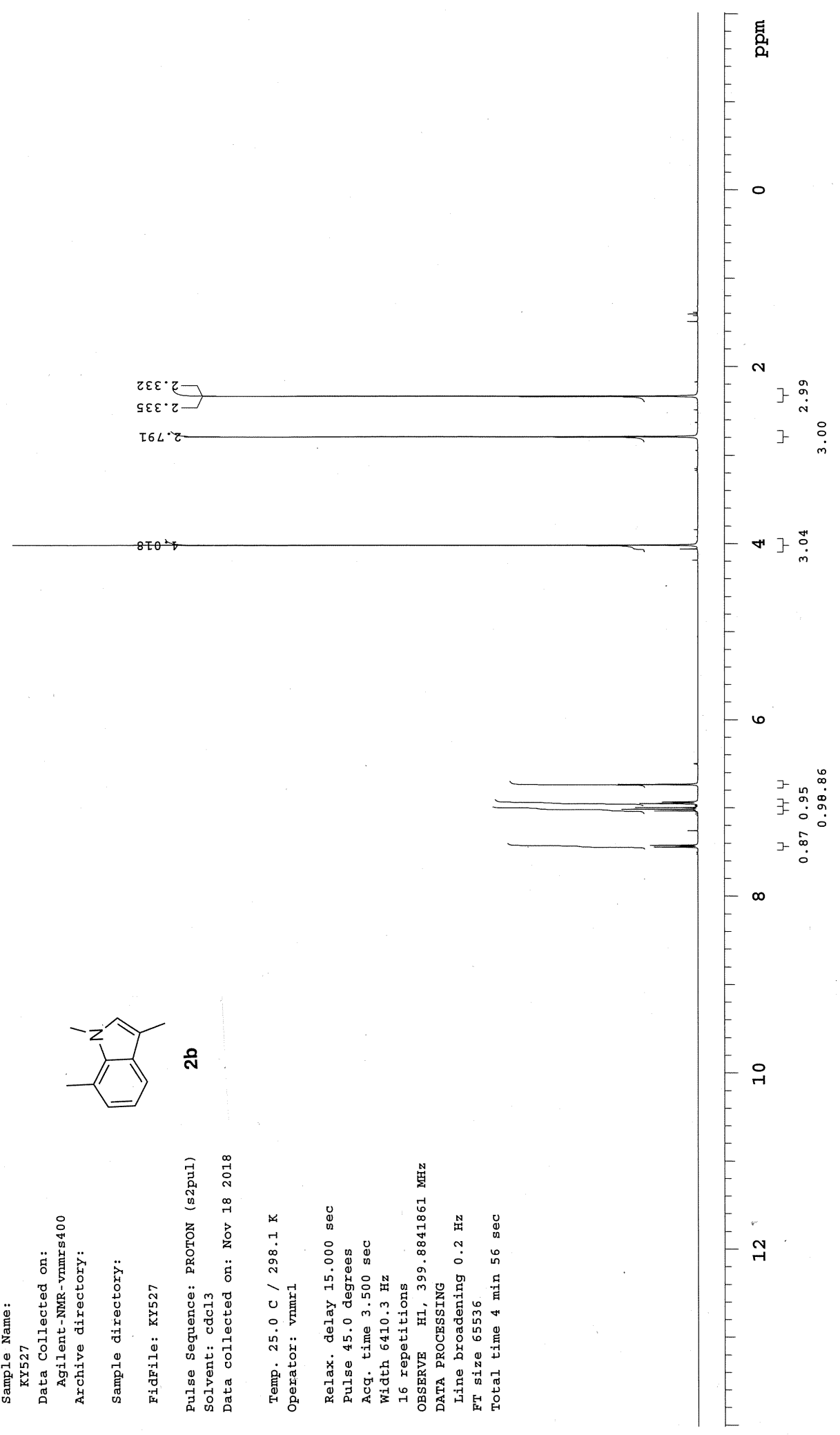

Figure S60. ${ }^{1} \mathrm{H}$ NMR Spectrum of $2 \mathbf{b}\left(400 \mathrm{MHz}, \mathrm{CDCl}_{3}\right)$ 


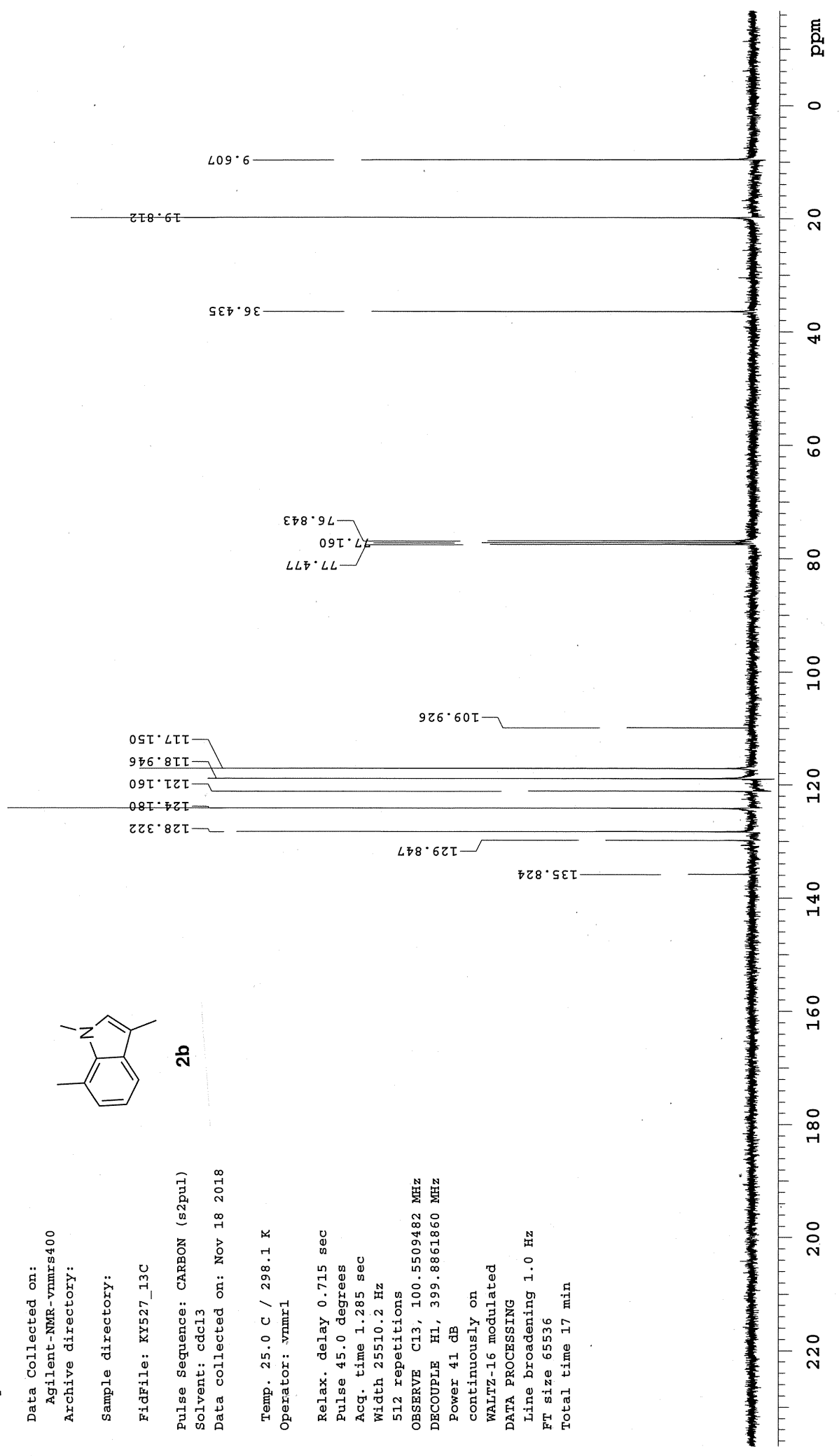

Figure S61. ${ }^{13} \mathrm{C}$ NMR Spectrum of $\mathbf{2 b}\left(101 \mathrm{MHz}, \mathrm{CDCl}_{3}\right)$ 


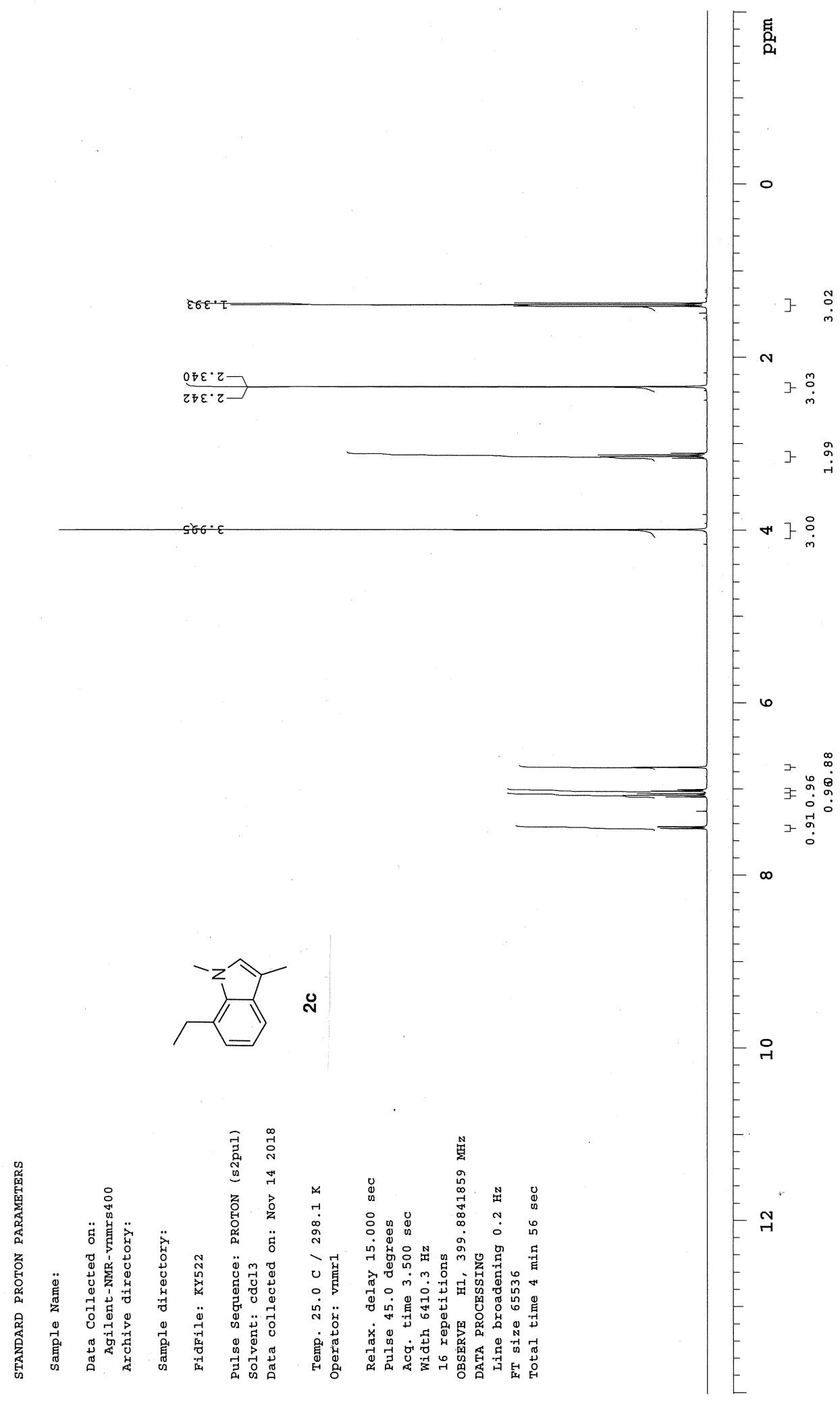

Figure S62. ${ }^{1} \mathrm{H}$ NMR Spectrum of 2 c $\left(400 \mathrm{MHz}, \mathrm{CDCl}_{3}\right)$ 


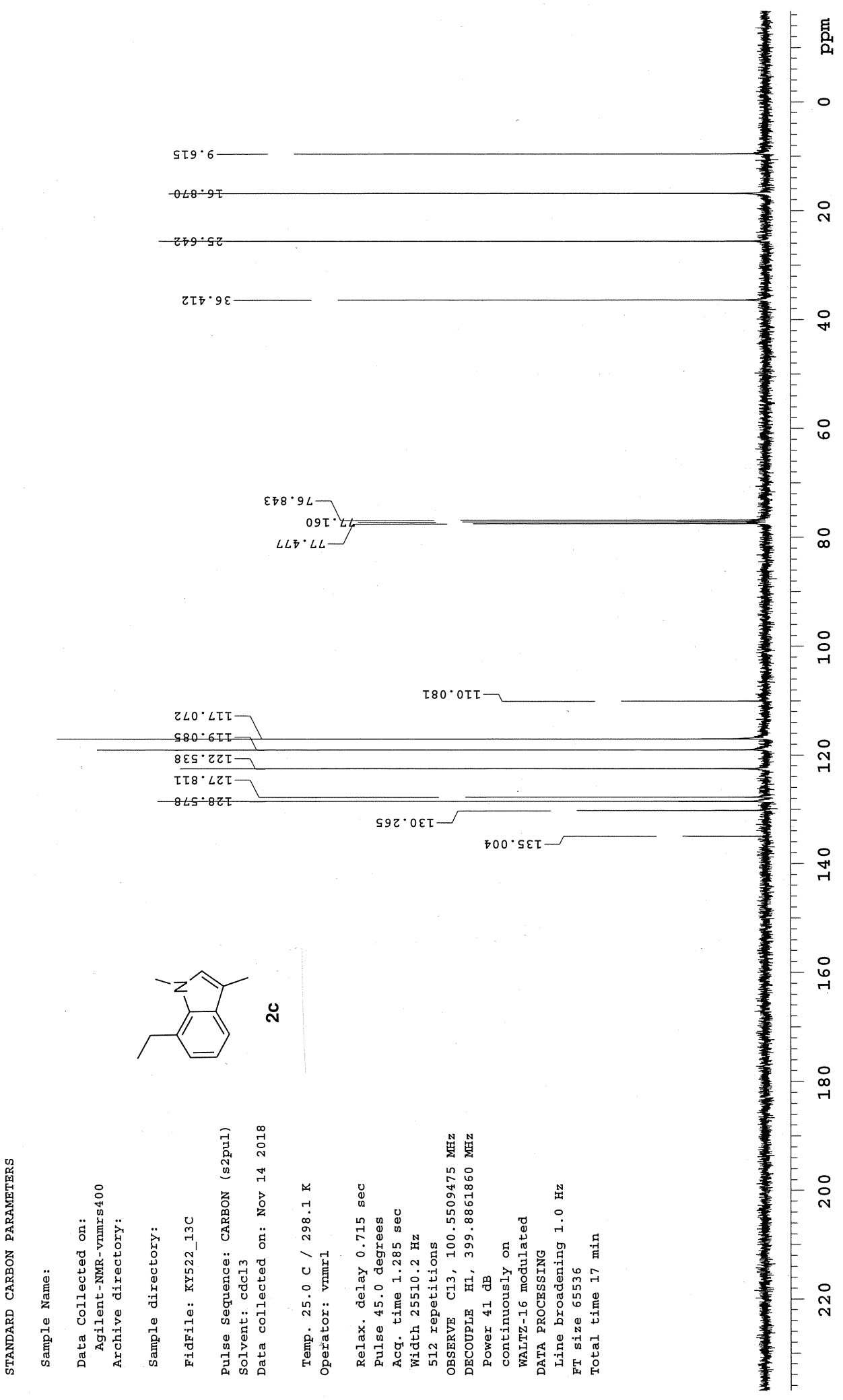

Figure S63. ${ }^{13} \mathrm{C}$ NMR Spectrum of 2c $\left(101 \mathrm{MHz}, \mathrm{CDCl}_{3}\right)$ 


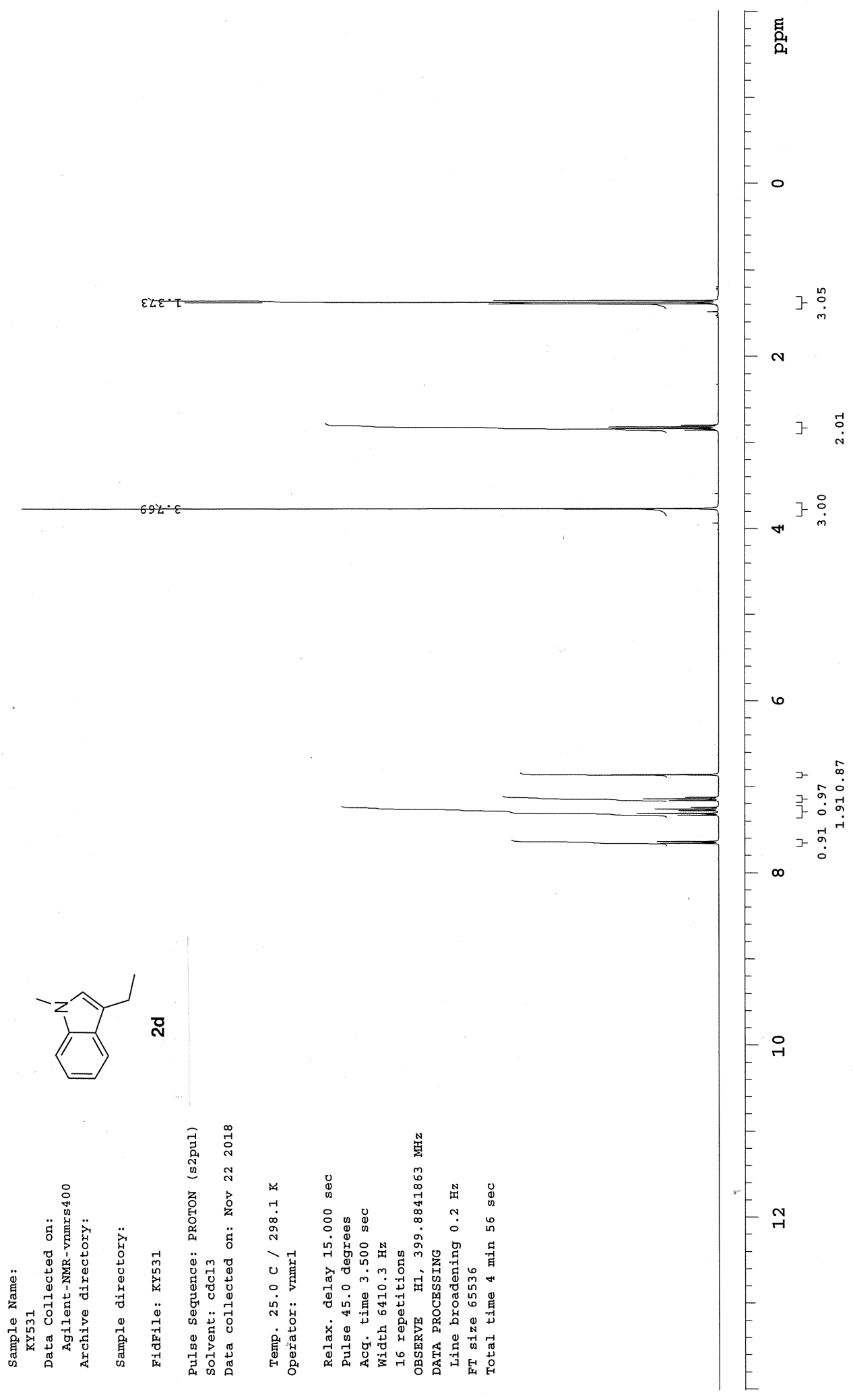

Figure S64. ${ }^{1} \mathrm{H}$ NMR Spectrum of $\mathbf{2 d}\left(400 \mathrm{MHz}, \mathrm{CDCl}_{3}\right)$ 


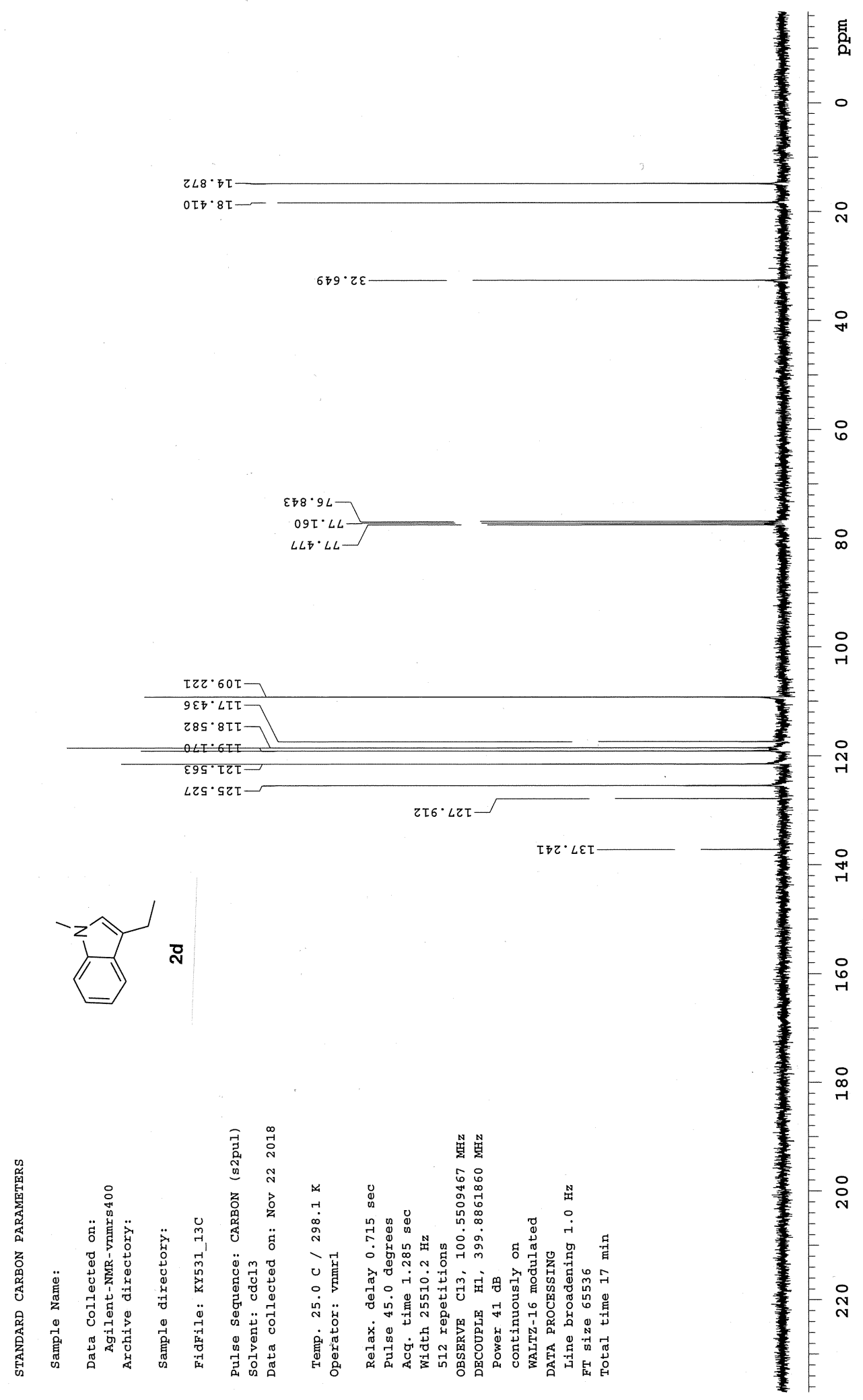

Figure S65. ${ }^{13} \mathrm{C}$ NMR Spectrum of $2 d\left(101 \mathrm{MHz}, \mathrm{CDCl}_{3}\right)$ 


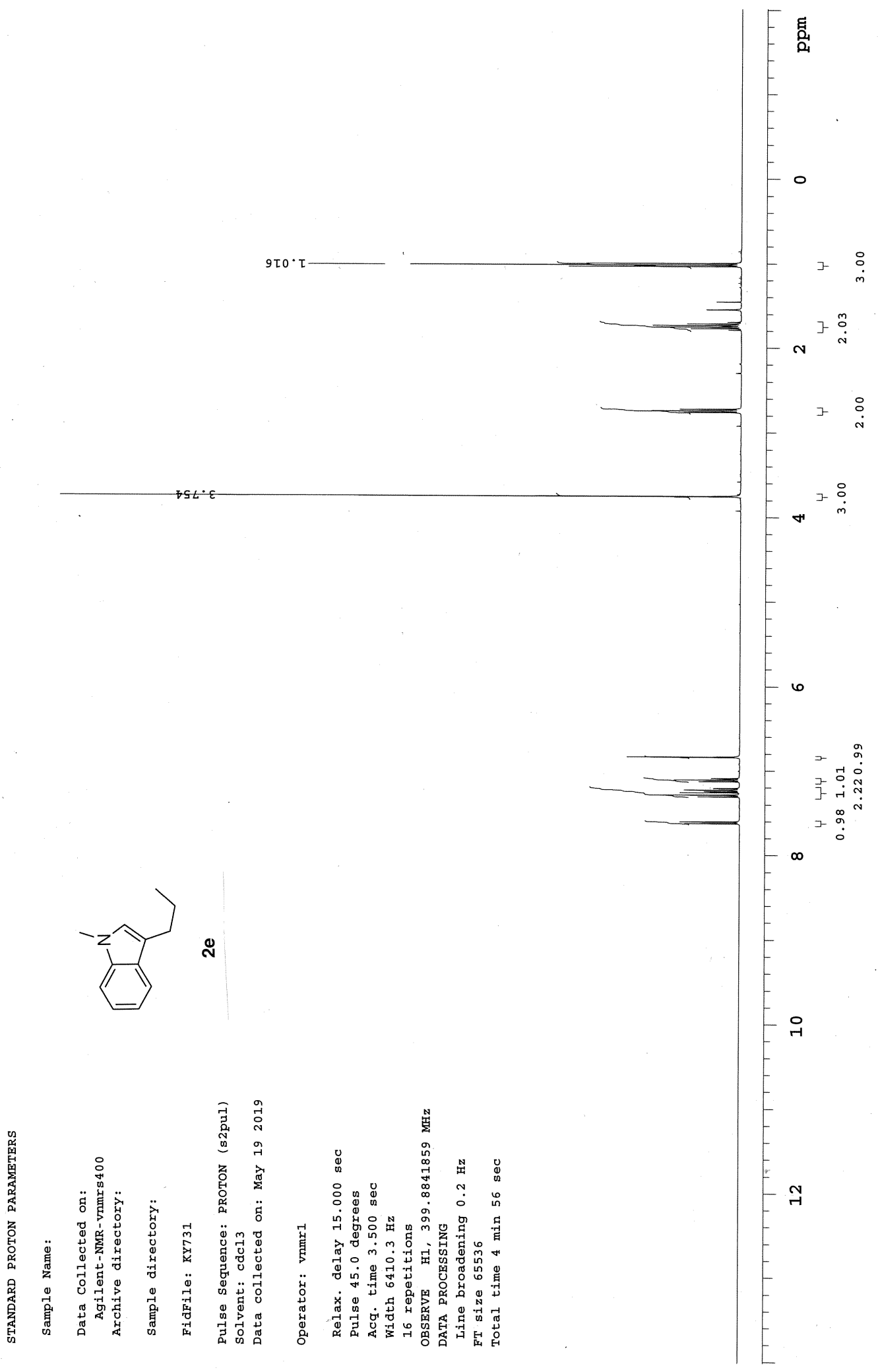

Figure S66. ${ }^{1} \mathrm{H}$ NMR Spectrum of 2 e $\left(400 \mathrm{MHz}, \mathrm{CDCl}_{3}\right)$ 


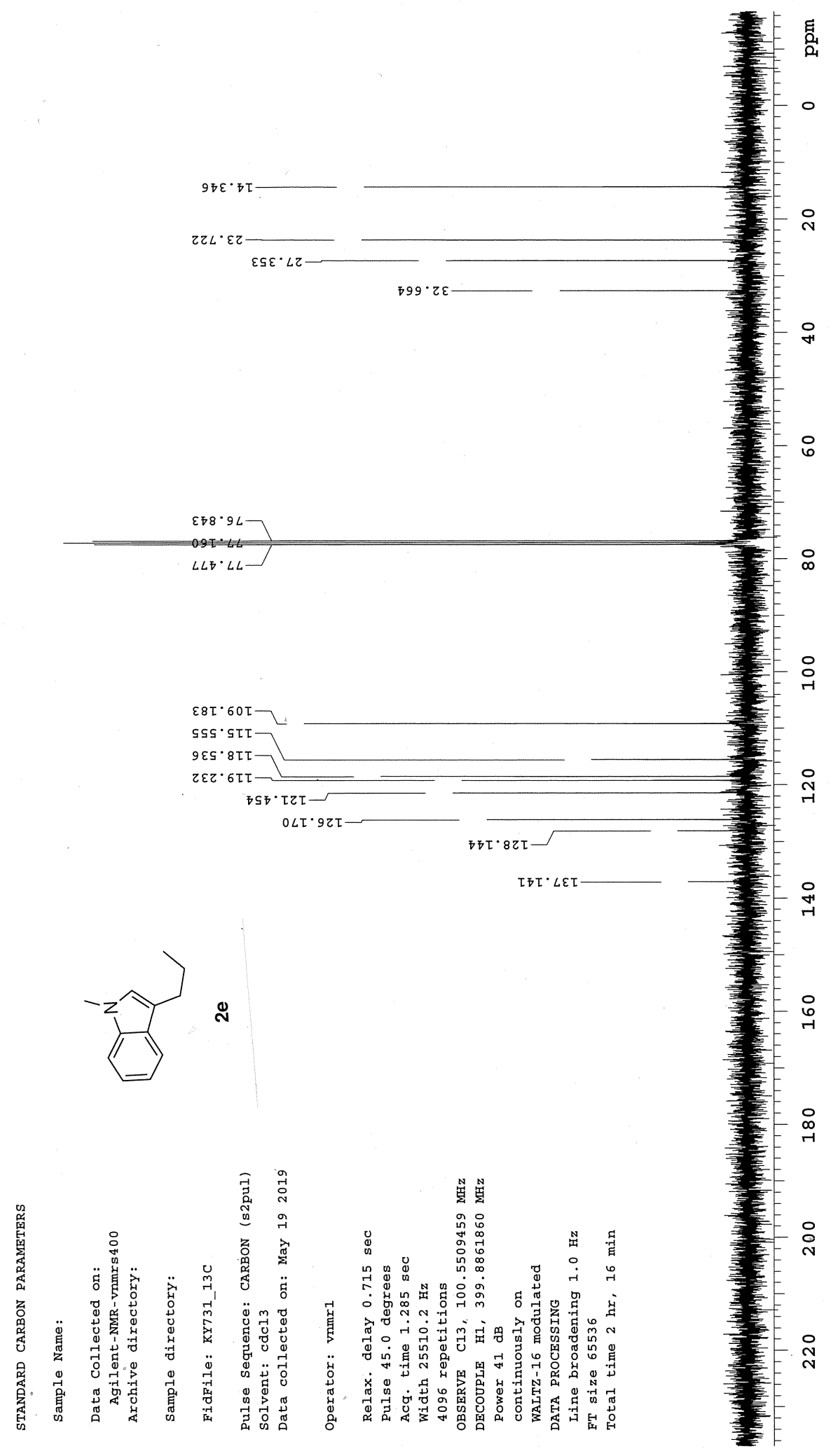

Figure S67. ${ }^{13} \mathrm{C}$ NMR Spectrum of 2 e $\left(101 \mathrm{MHz}, \mathrm{CDCl}_{3}\right)$ 


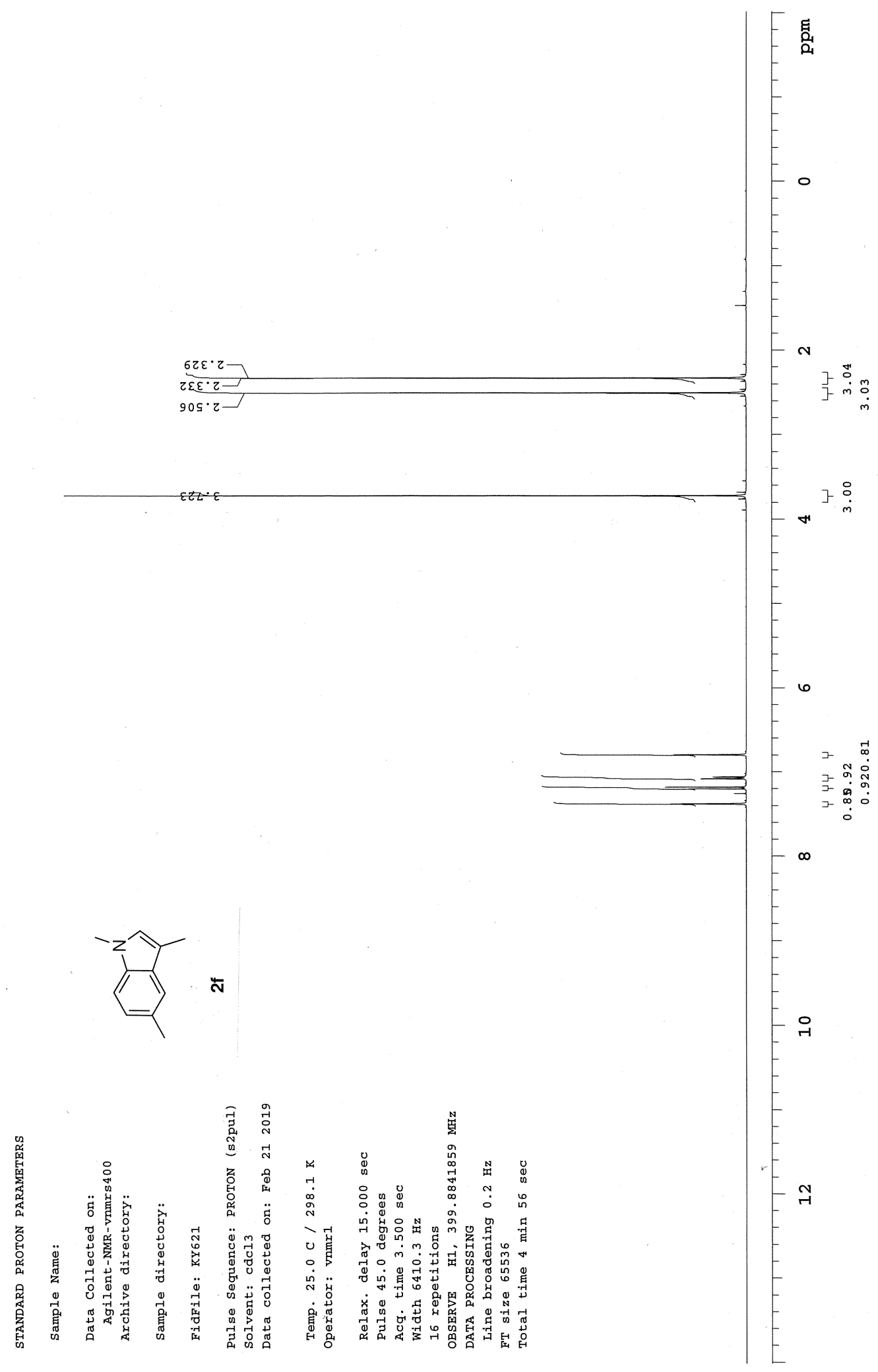

Figure S68. ${ }^{1} \mathrm{H}$ NMR Spectrum of $2 \mathbf{f}\left(400 \mathrm{MHz}, \mathrm{CDCl}_{3}\right)$ 


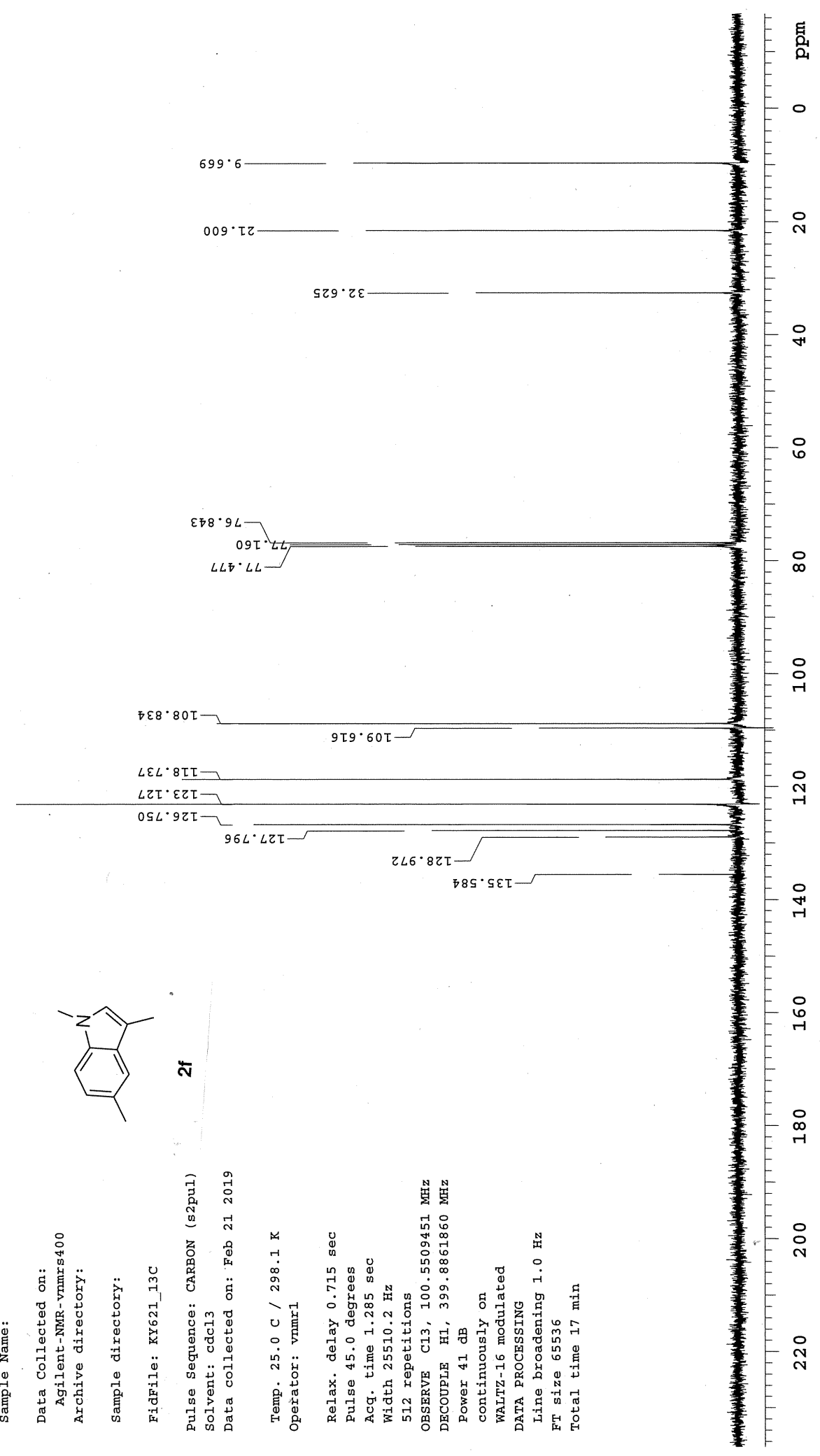

Figure S69. ${ }^{13} \mathrm{C}$ NMR Spectrum of $2 \mathbf{f}\left(101 \mathrm{MHz}, \mathrm{CDCl}_{3}\right)$ 


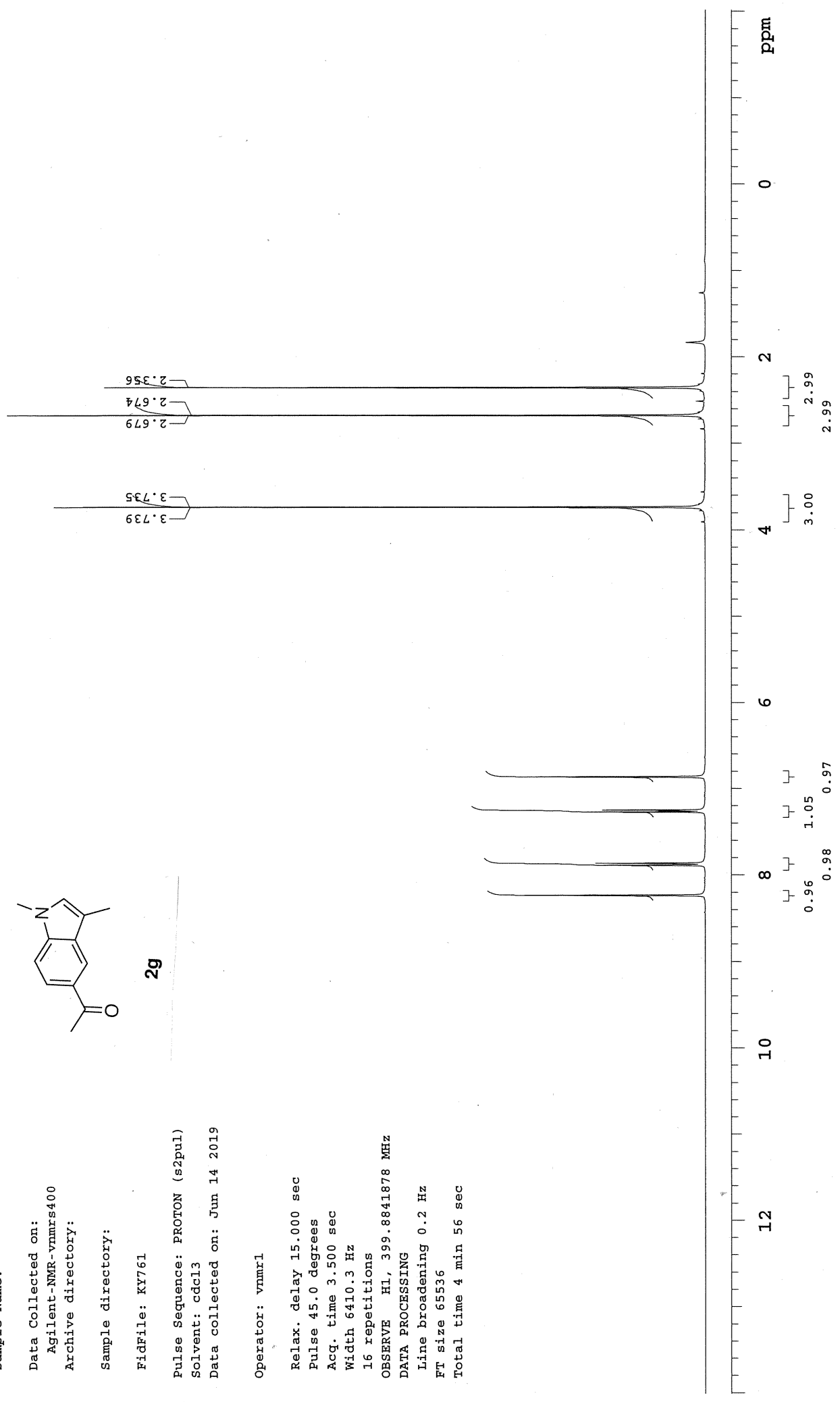

Figure S70. ${ }^{1} \mathrm{H}$ NMR Spectrum of $2 \mathrm{~g}\left(400 \mathrm{MHz}, \mathrm{CDCl}_{3}\right)$ 


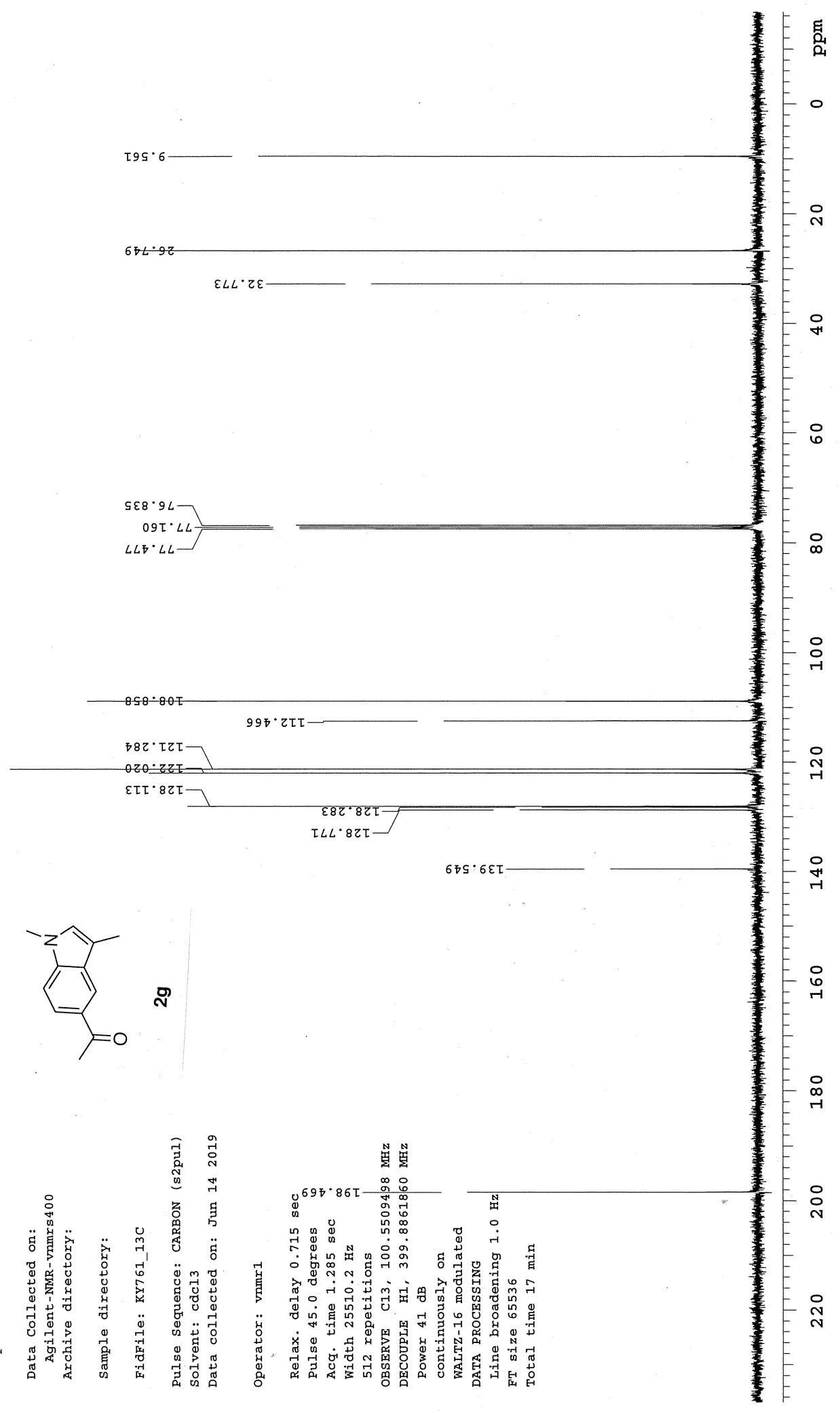

Figure S71. ${ }^{13} \mathrm{C}$ NMR Spectrum of $\mathbf{2 g}\left(101 \mathrm{MHz}, \mathrm{CDCl}_{3}\right)$ 


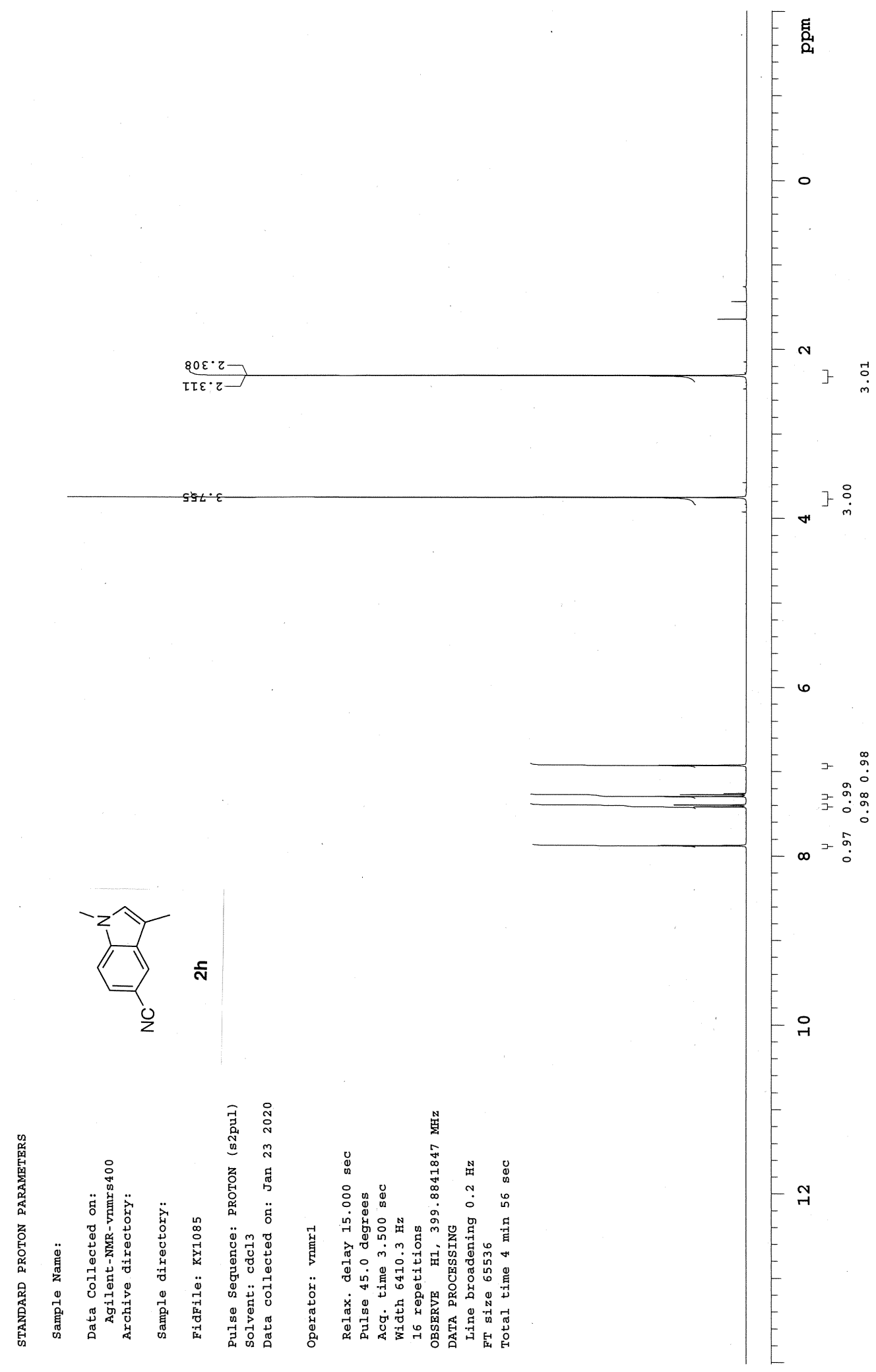

Figure S72. ${ }^{1} \mathrm{H}$ NMR Spectrum of $\mathbf{2 h}\left(400 \mathrm{MHz}, \mathrm{CDCl}_{3}\right)$ 


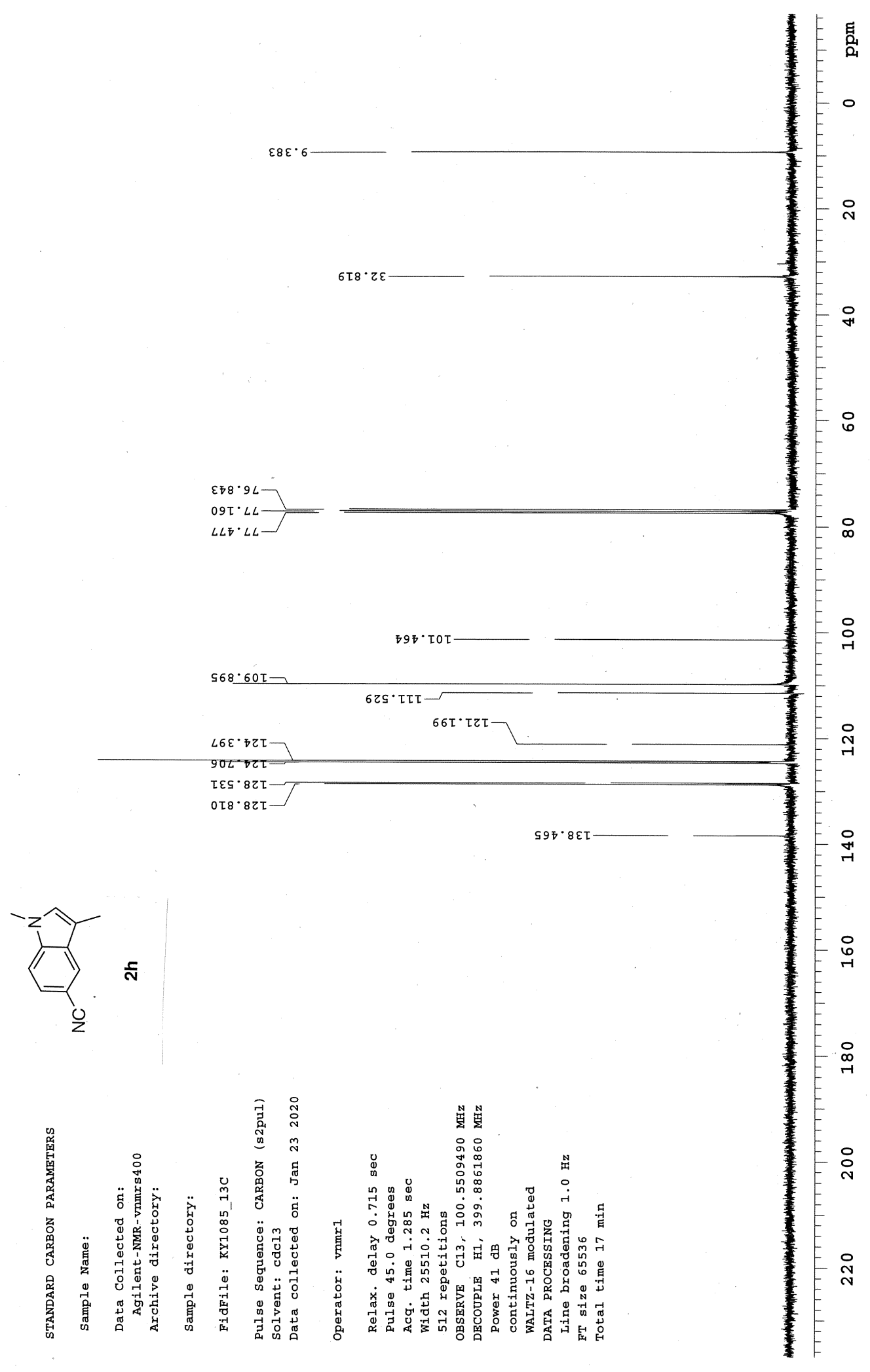

Figure S73. ${ }^{13} \mathrm{C}$ NMR Spectrum of $\mathbf{2 h}\left(101 \mathrm{MHz}, \mathrm{CDCl}_{3}\right)$ 


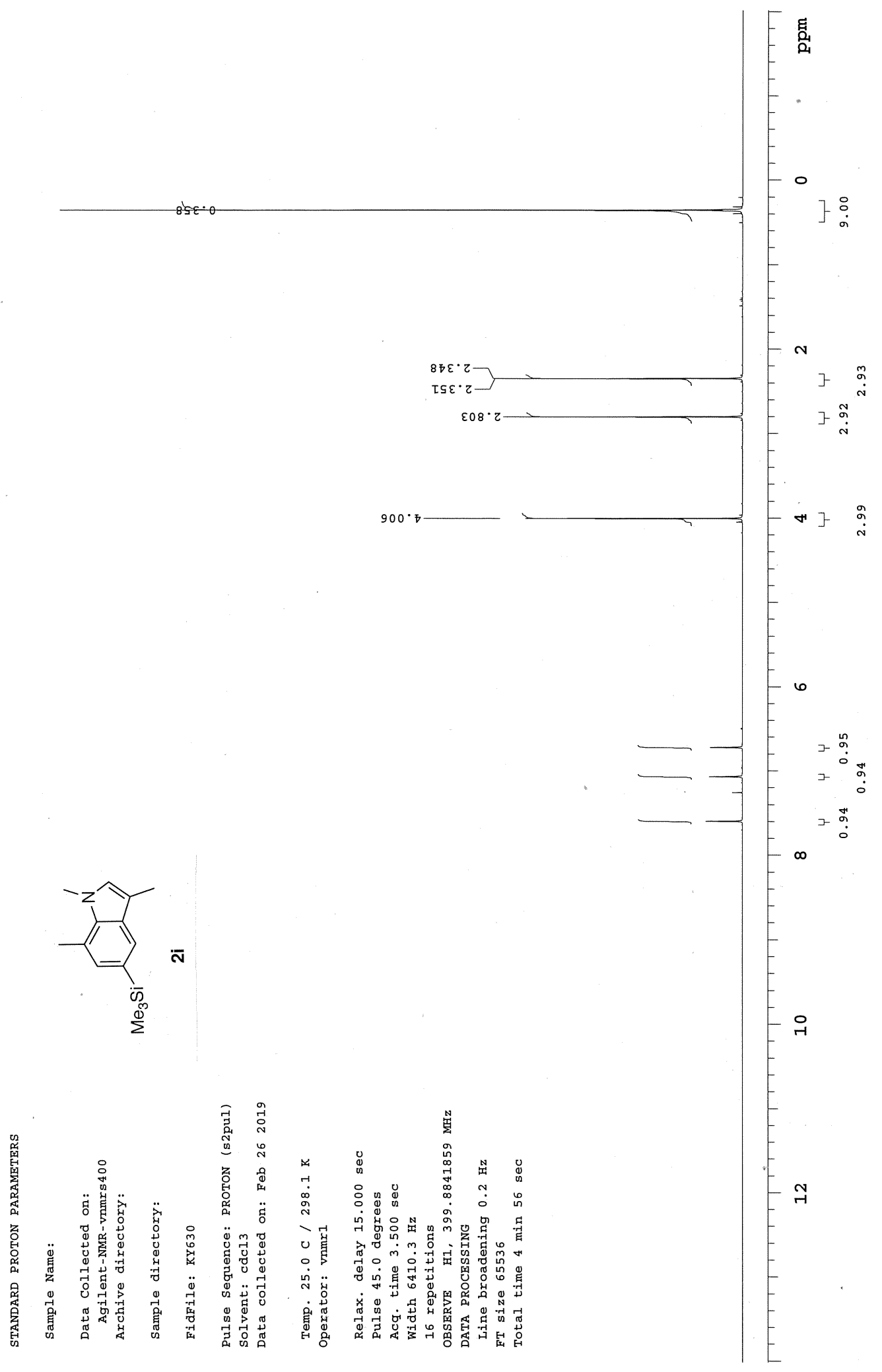

Figure S74. ${ }^{1} \mathrm{H}$ NMR Spectrum of $2 \mathbf{i}\left(400 \mathrm{MHz}, \mathrm{CDCl}_{3}\right)$ 


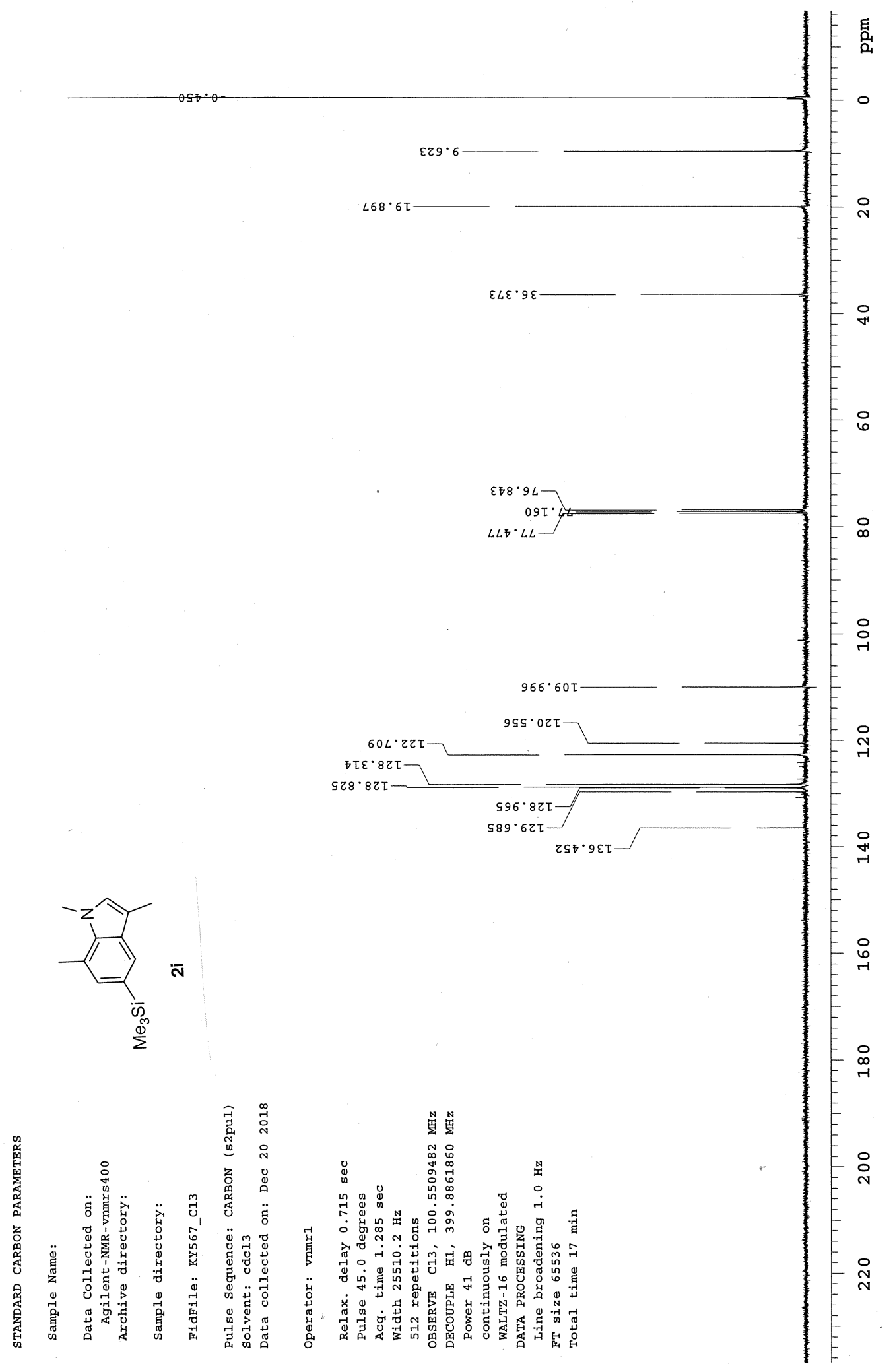

Figure S75. ${ }^{13} \mathrm{C}$ NMR Spectrum of $2 \mathbf{i}\left(101 \mathrm{MHz}, \mathrm{CDCl}_{3}\right)$ 


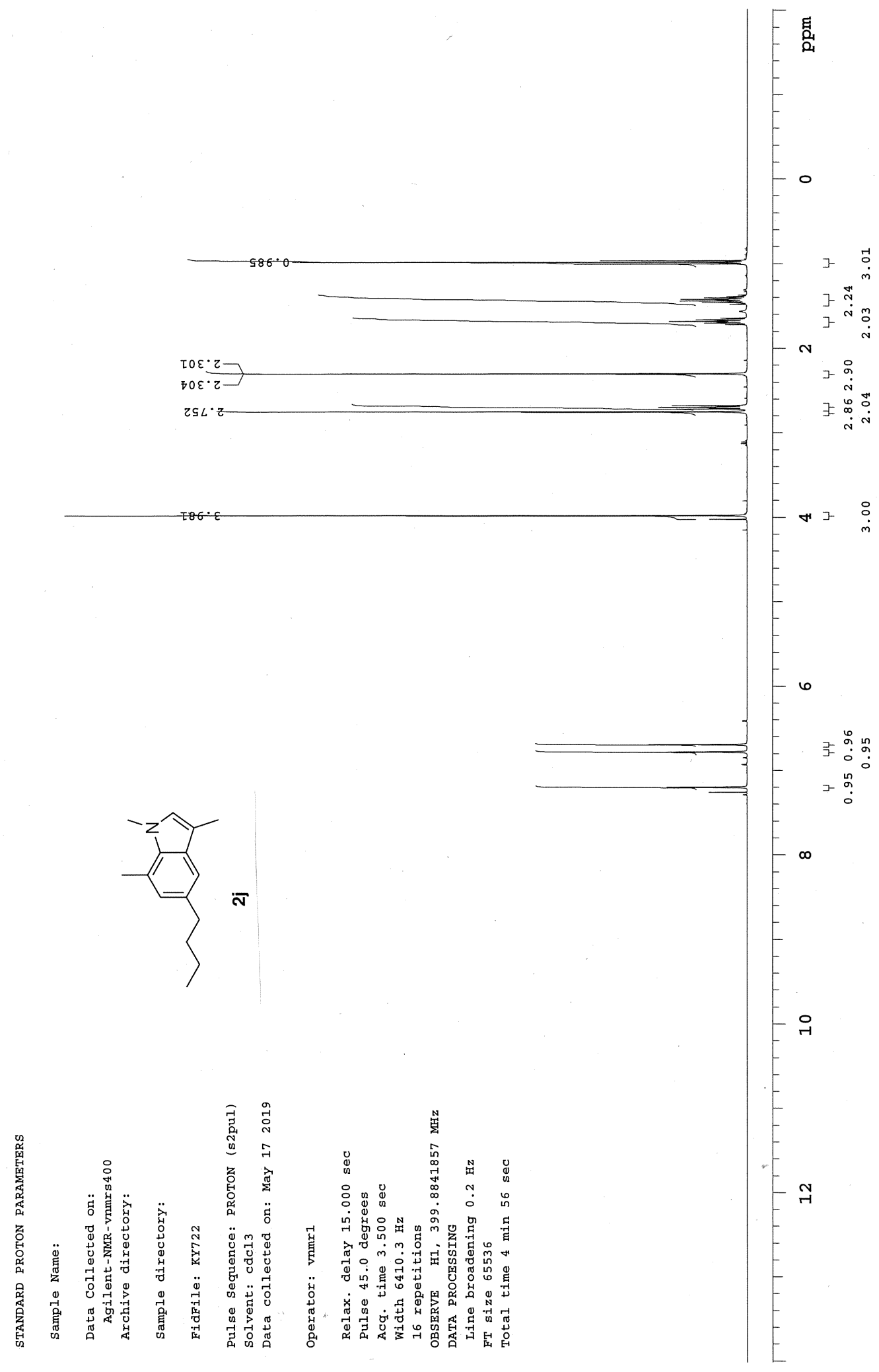

Figure S76. ${ }^{1} \mathrm{H}$ NMR Spectrum of $\mathbf{2} \mathbf{j}\left(400 \mathrm{MHz}, \mathrm{CDCl}_{3}\right)$ 


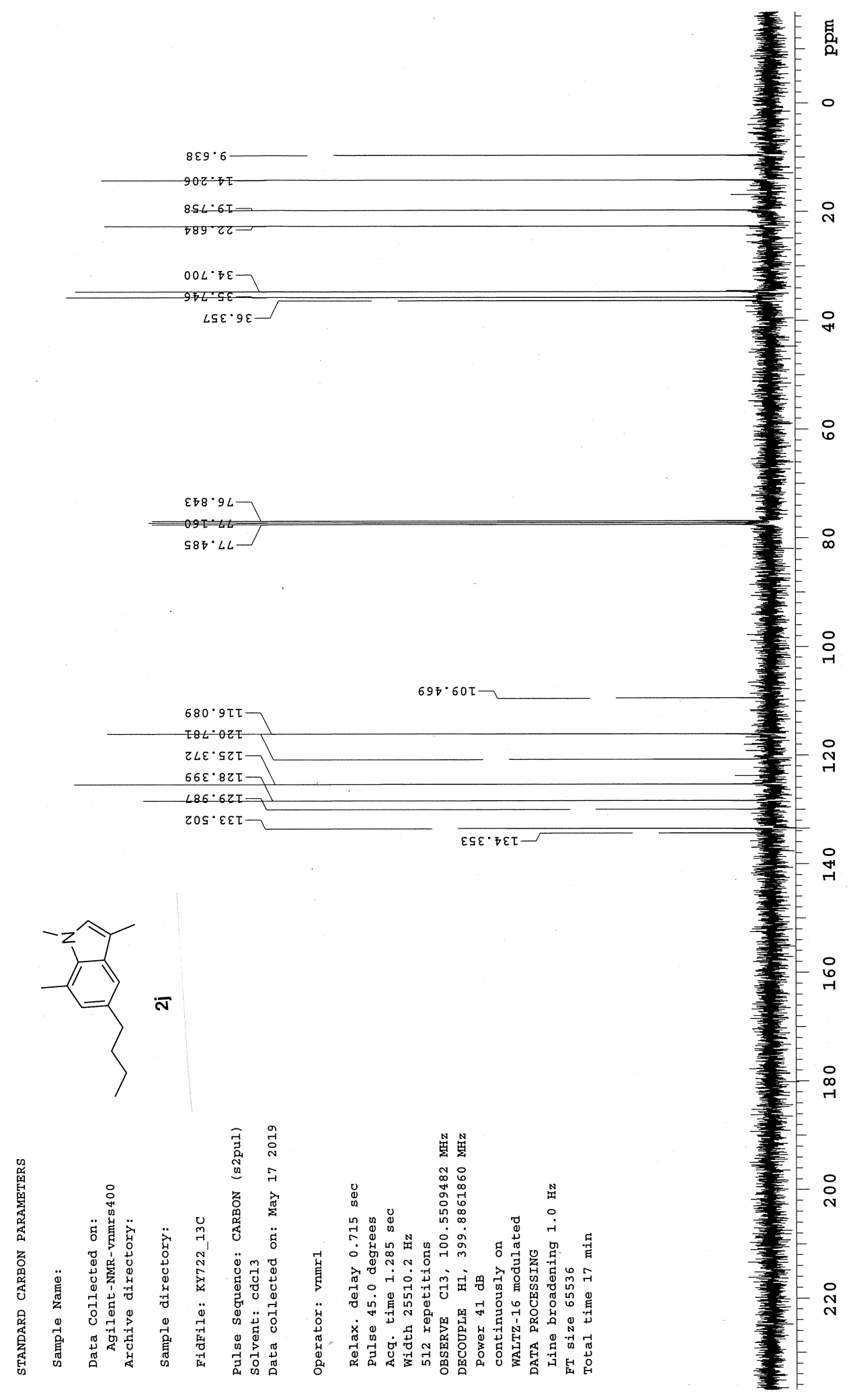

Figure S77. ${ }^{13} \mathrm{C}$ NMR Spectrum of $\mathbf{2} \mathbf{j}\left(101 \mathrm{MHz}, \mathrm{CDCl}_{3}\right)$ 


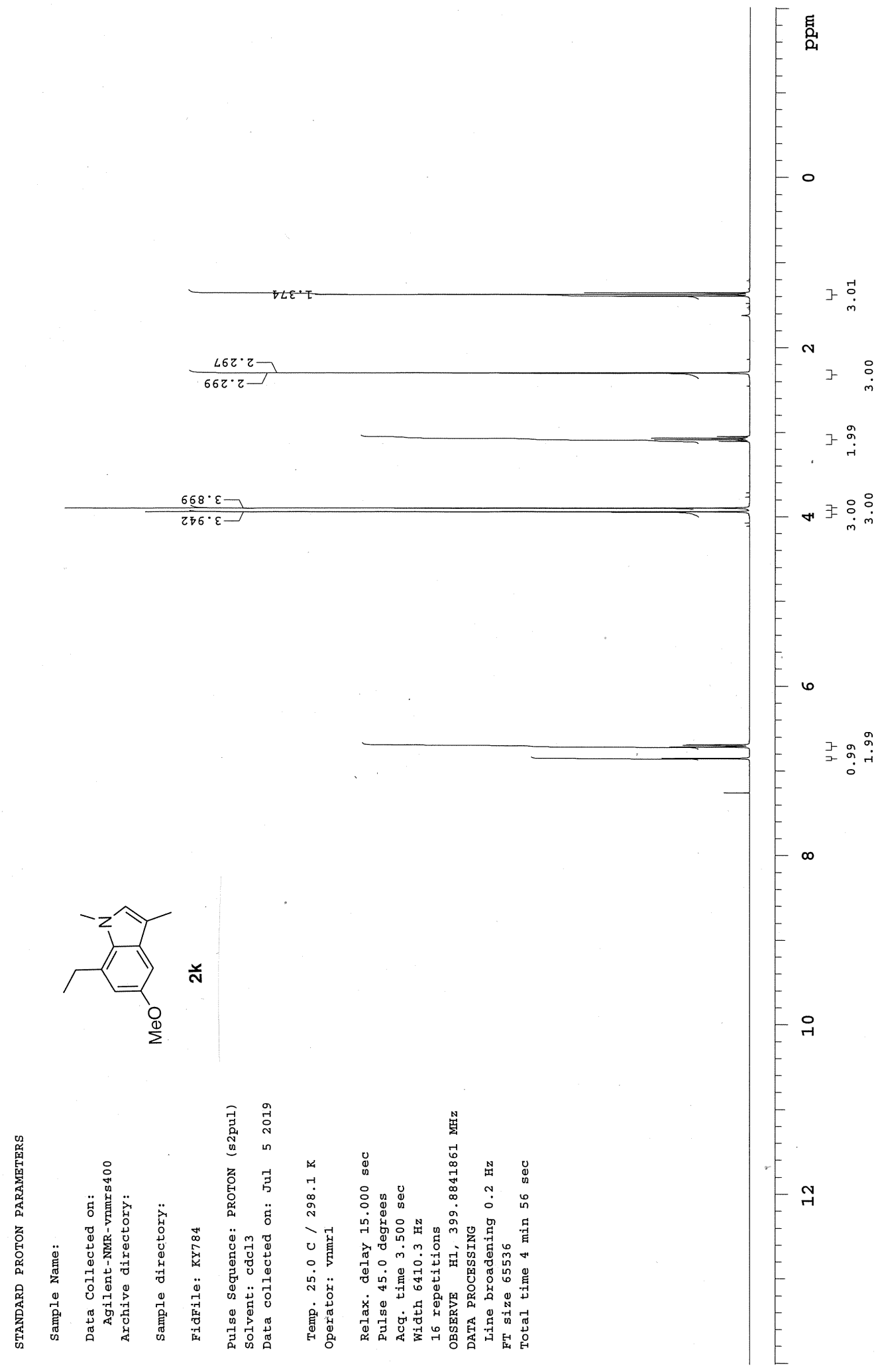

Figure S78. ${ }^{1} \mathrm{H}$ NMR Spectrum of $\mathbf{2 k}\left(400 \mathrm{MHz}, \mathrm{CDCl}_{3}\right)$ 


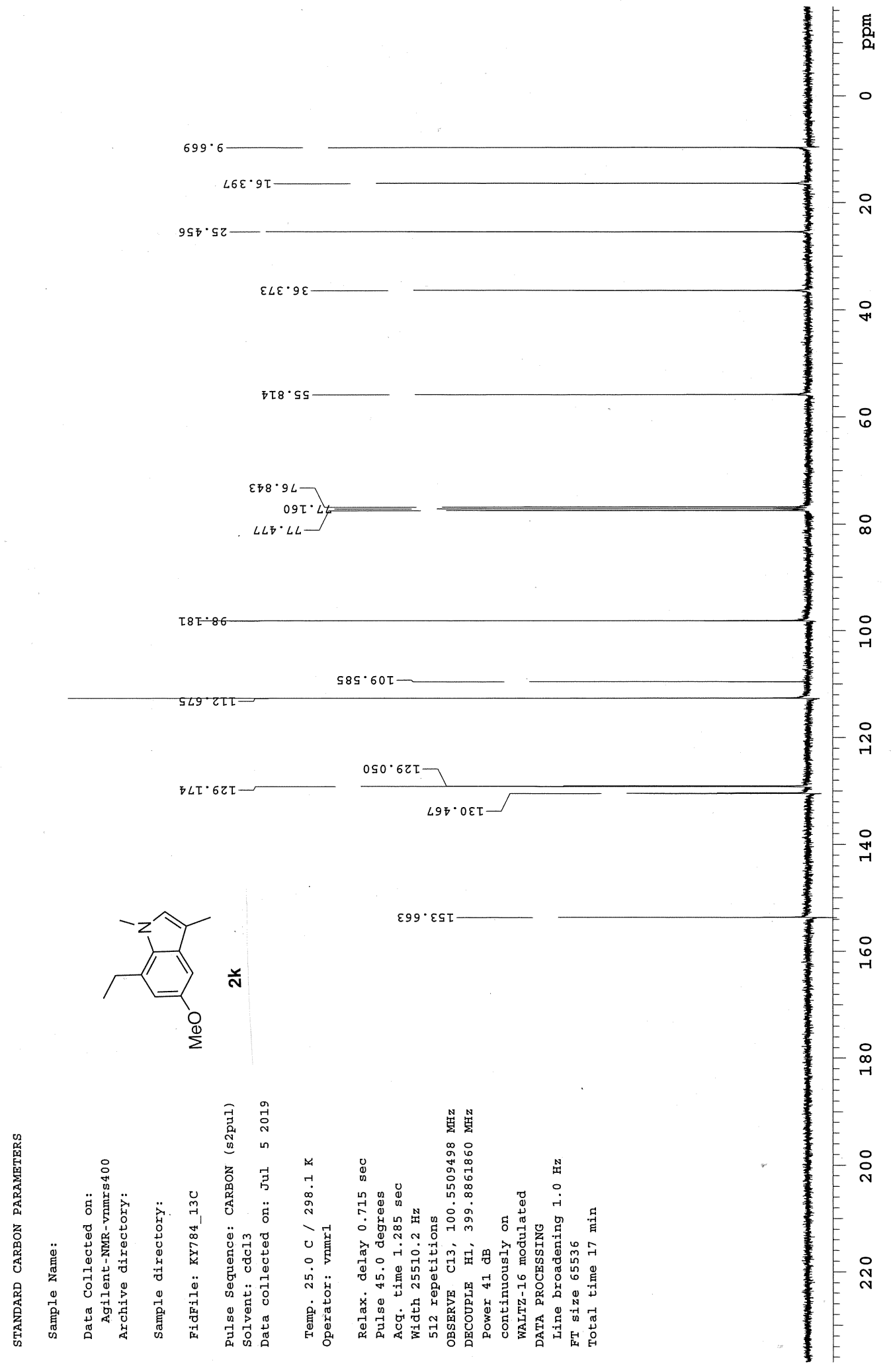

Figure S79. ${ }^{13} \mathrm{C}$ NMR Spectrum of $\mathbf{2 k}\left(101 \mathrm{MHz}, \mathrm{CDCl}_{3}\right)$ 


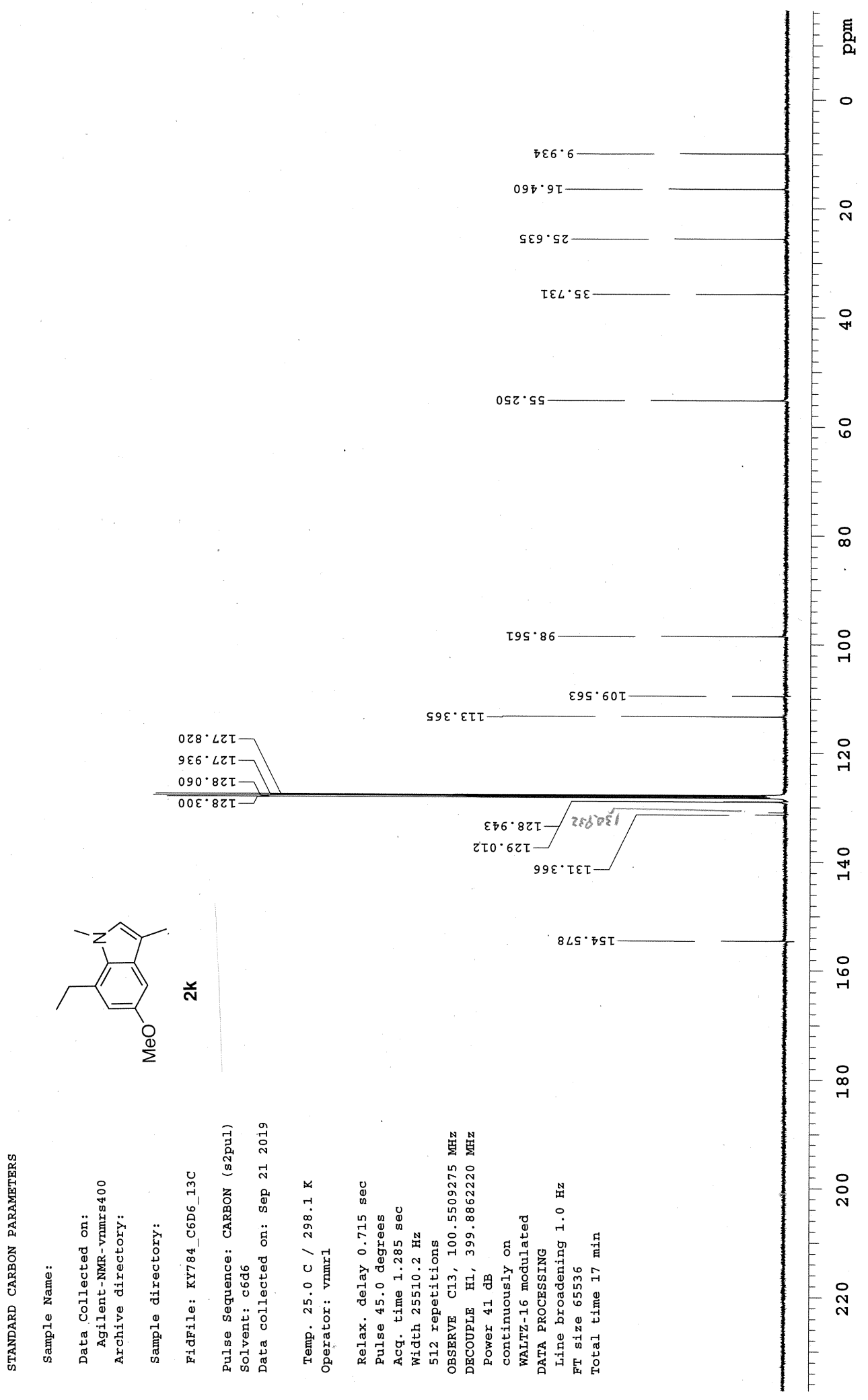

Figure S80. ${ }^{13} \mathrm{C}$ NMR Spectrum of $\mathbf{2 k}\left(101 \mathrm{MHz}, \mathrm{C}_{6} \mathrm{D}_{6}\right)$ 


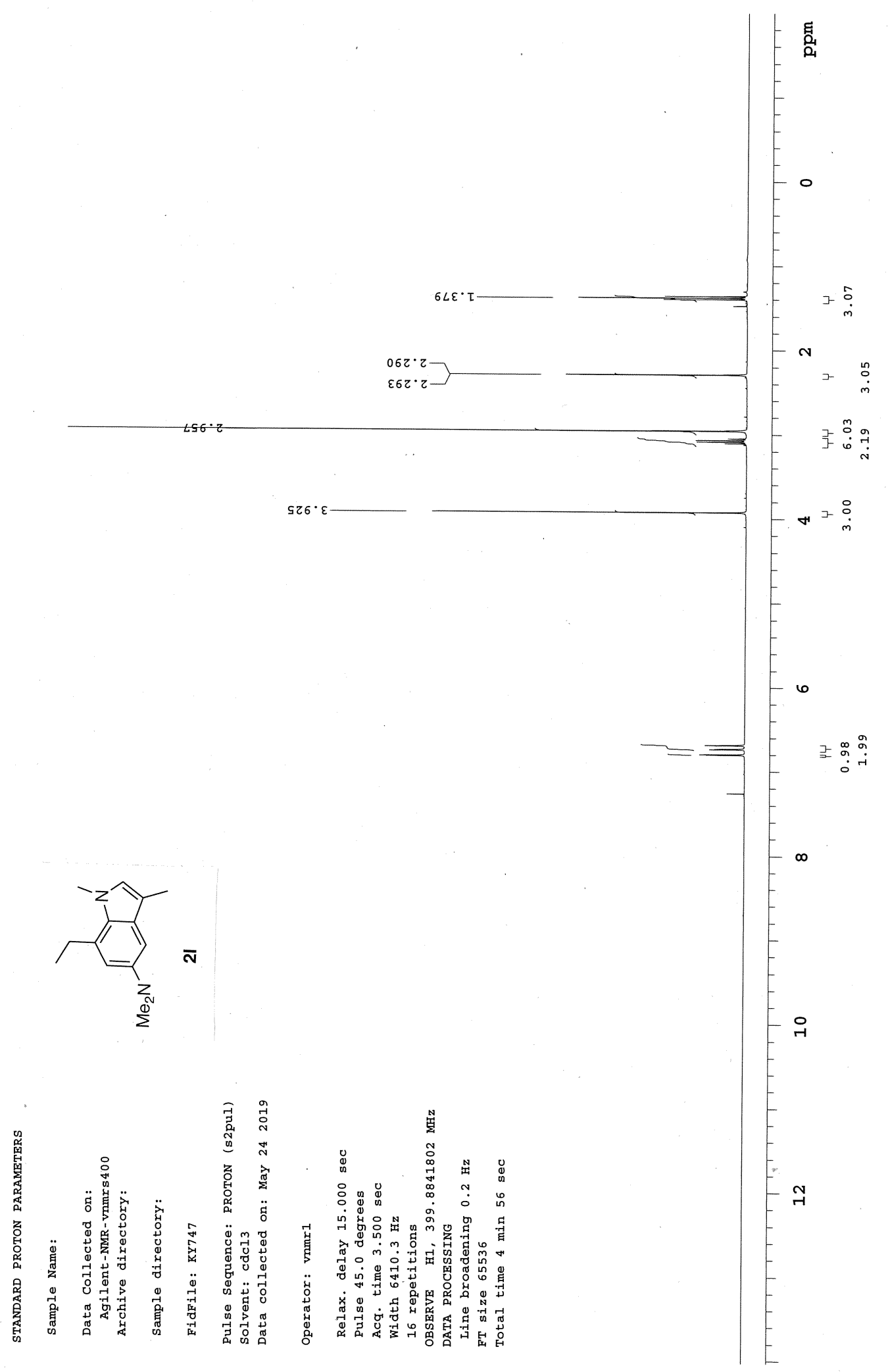

Figure S81. ${ }^{1} \mathrm{H}$ NMR Spectrum of $21\left(400 \mathrm{MHz}, \mathrm{CDCl}_{3}\right)$ 


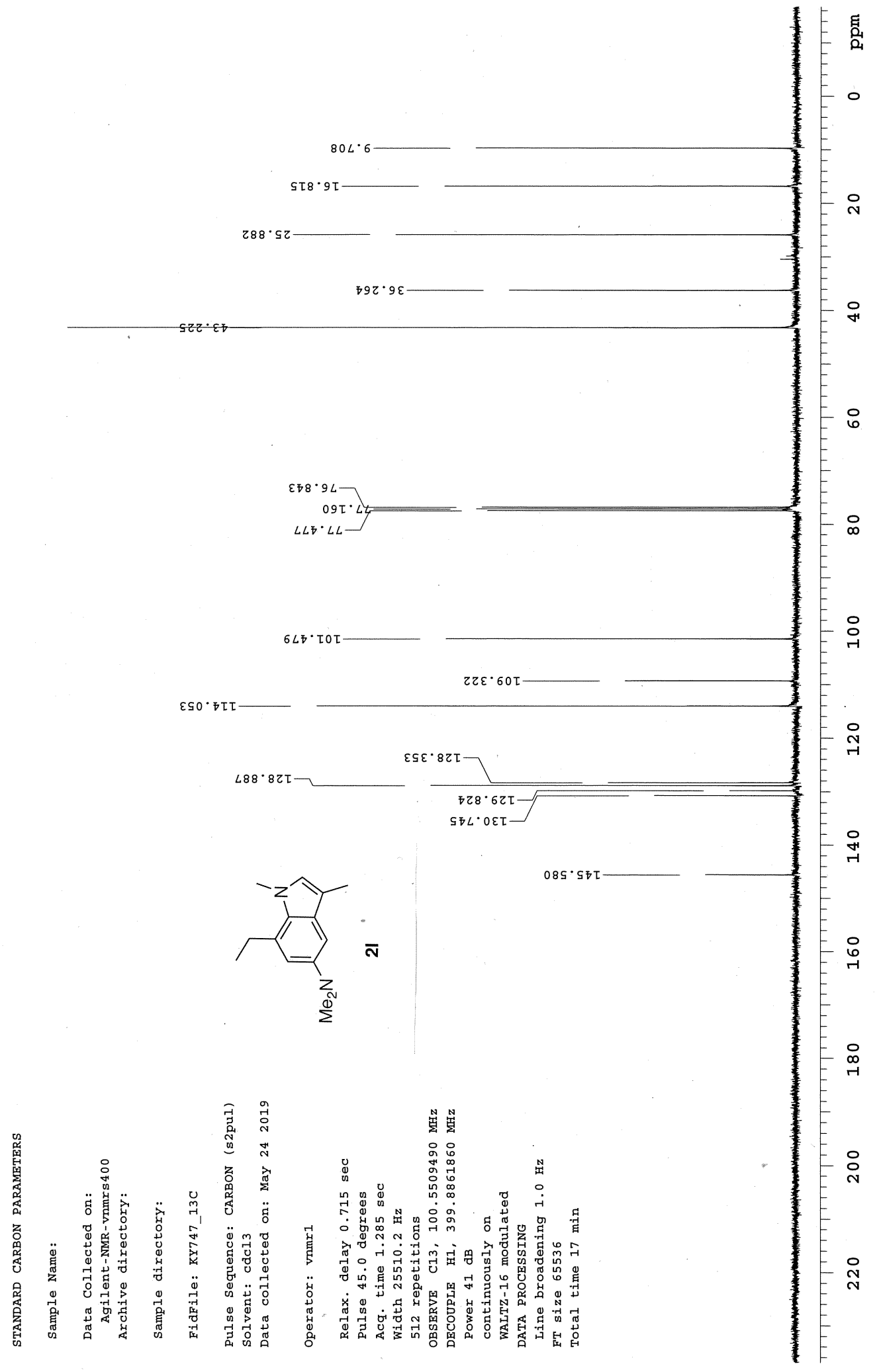

Figure S82. ${ }^{13} \mathrm{C}$ NMR Spectrum of $21\left(101 \mathrm{MHz}, \mathrm{CDCl}_{3}\right)$ 


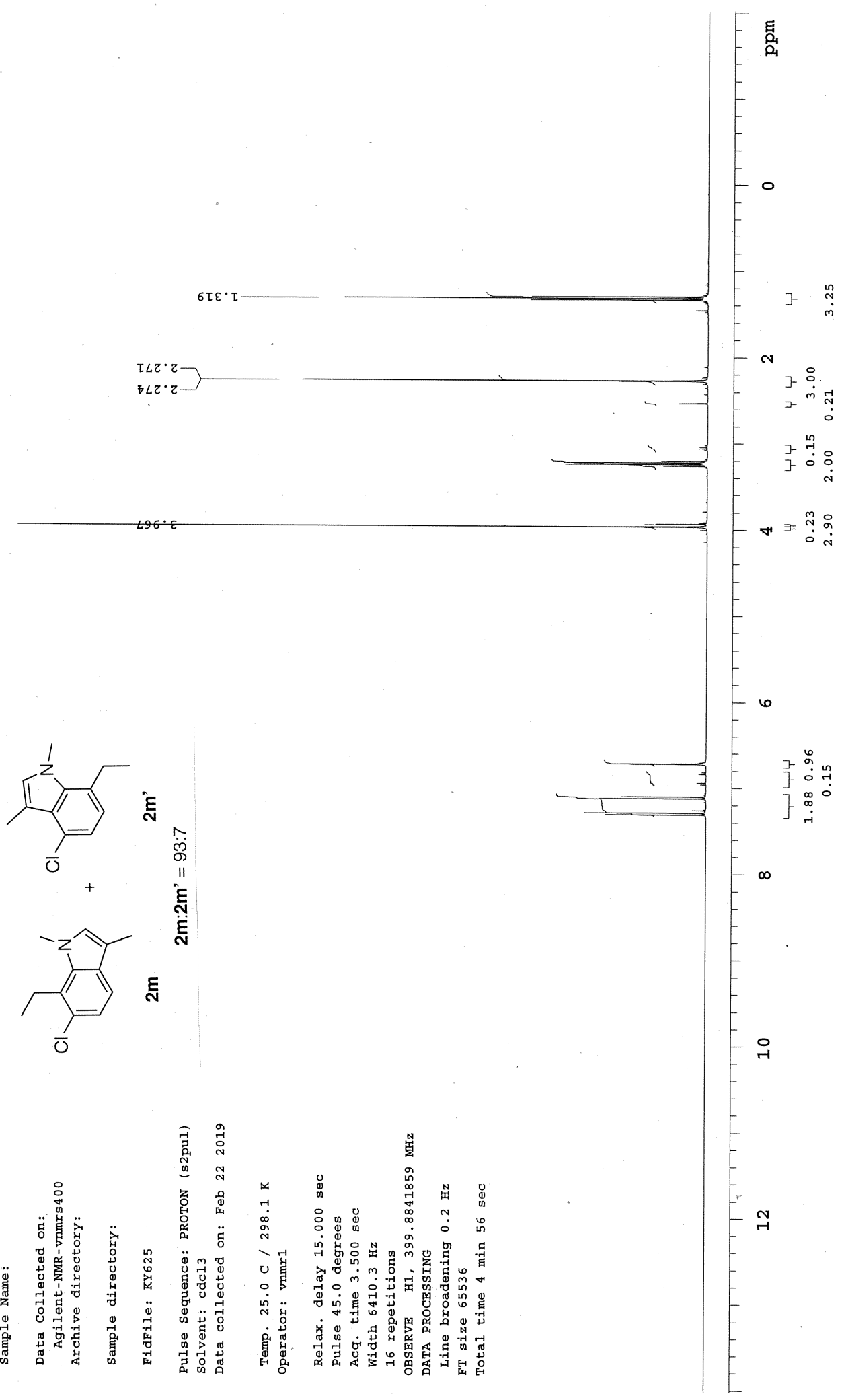

Figure S83. ${ }^{1} \mathrm{H}$ NMR Spectrum of $\mathbf{2 m}\left(400 \mathrm{MHz}, \mathrm{CDCl}_{3}\right)$ 


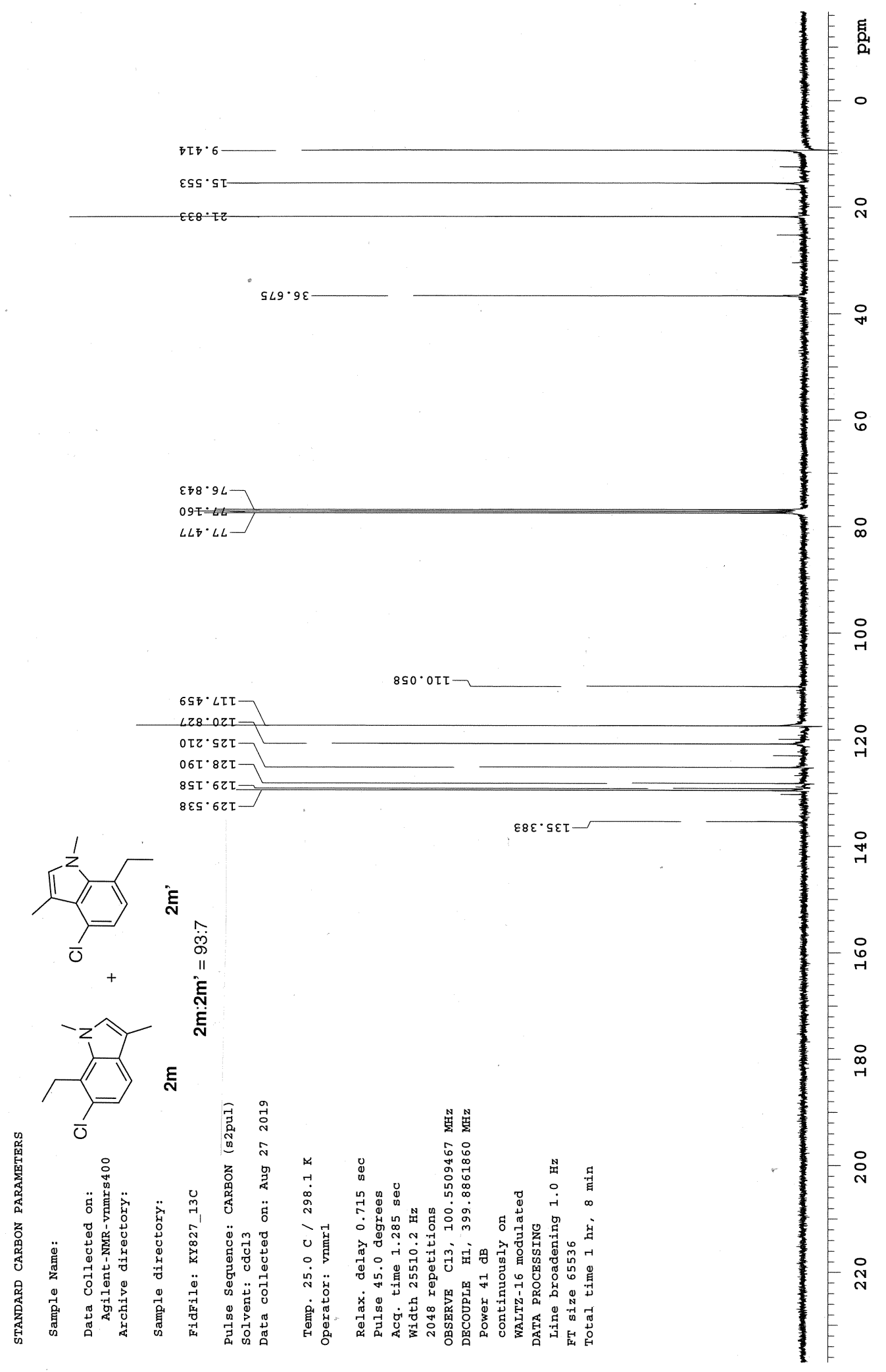

Figure S84. ${ }^{13} \mathrm{C}$ NMR Spectrum of $\mathbf{2 m}\left(101 \mathrm{MHz}, \mathrm{CDCl}_{3}\right)$ 


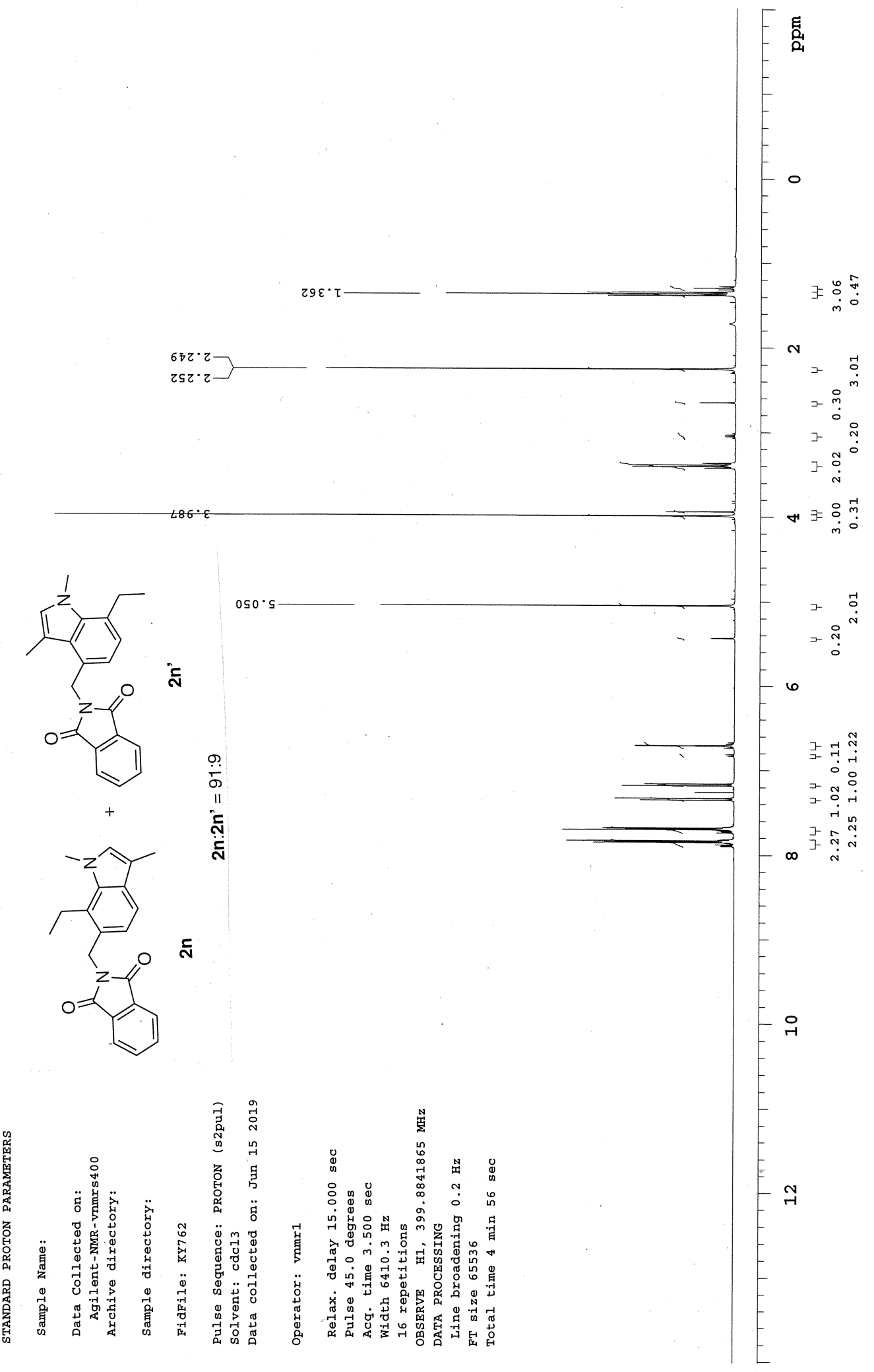

Figure S85. ${ }^{1} \mathrm{H}$ NMR Spectrum of 2 n $\left(400 \mathrm{MHz}, \mathrm{CDCl}_{3}\right)$ 

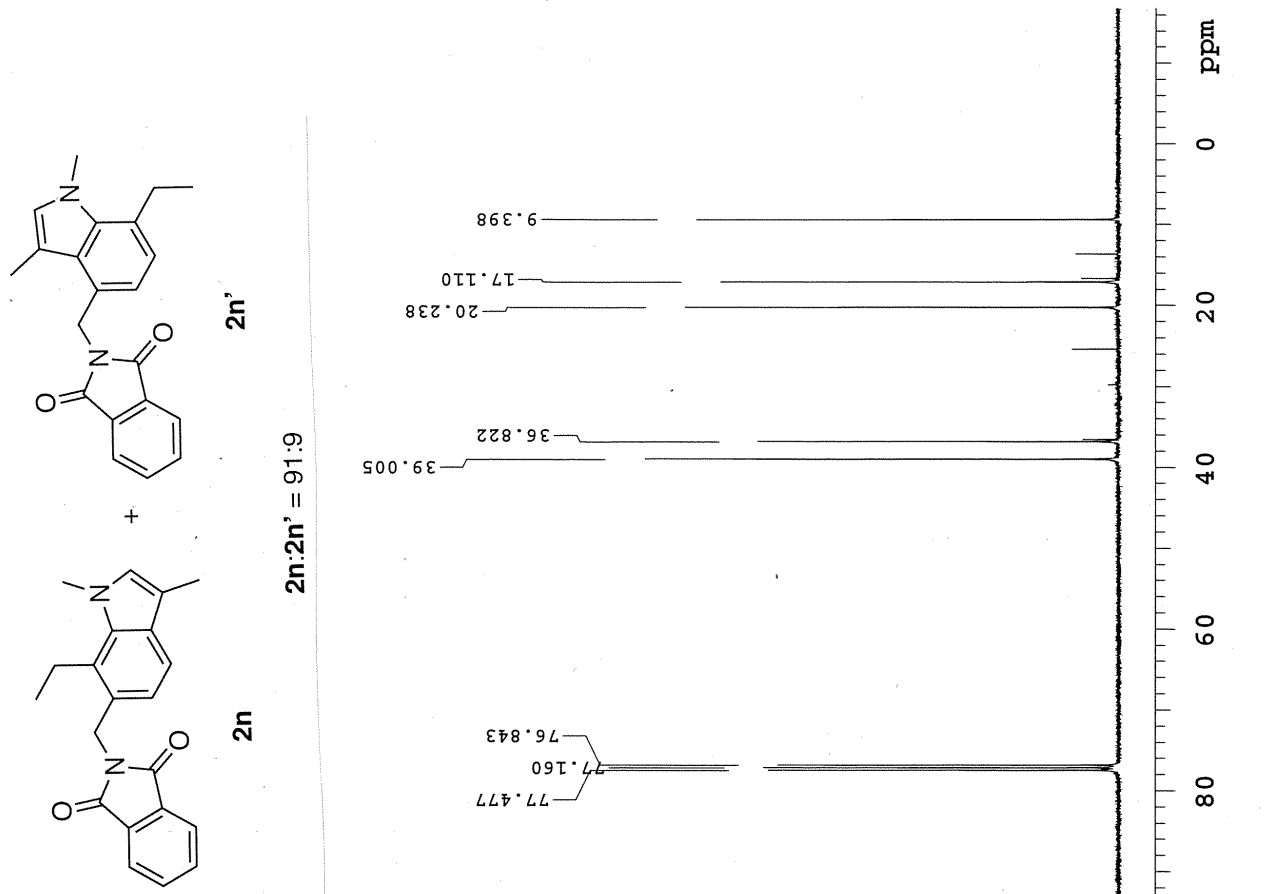

아

సั
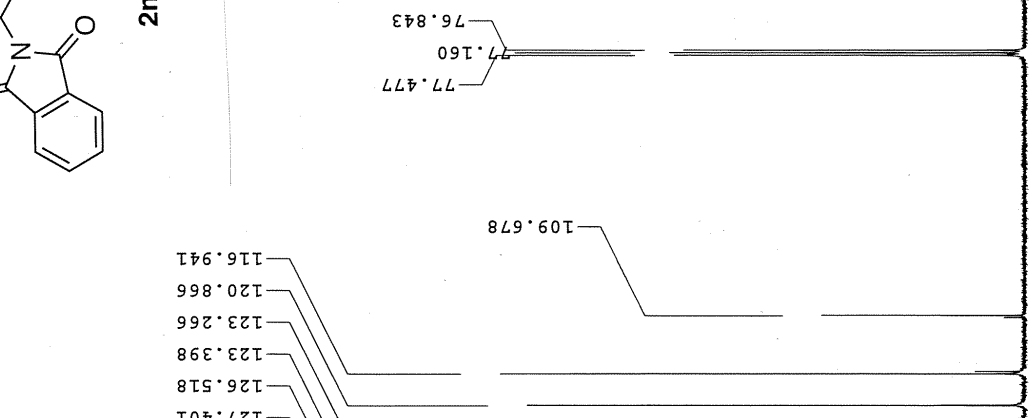

656. 


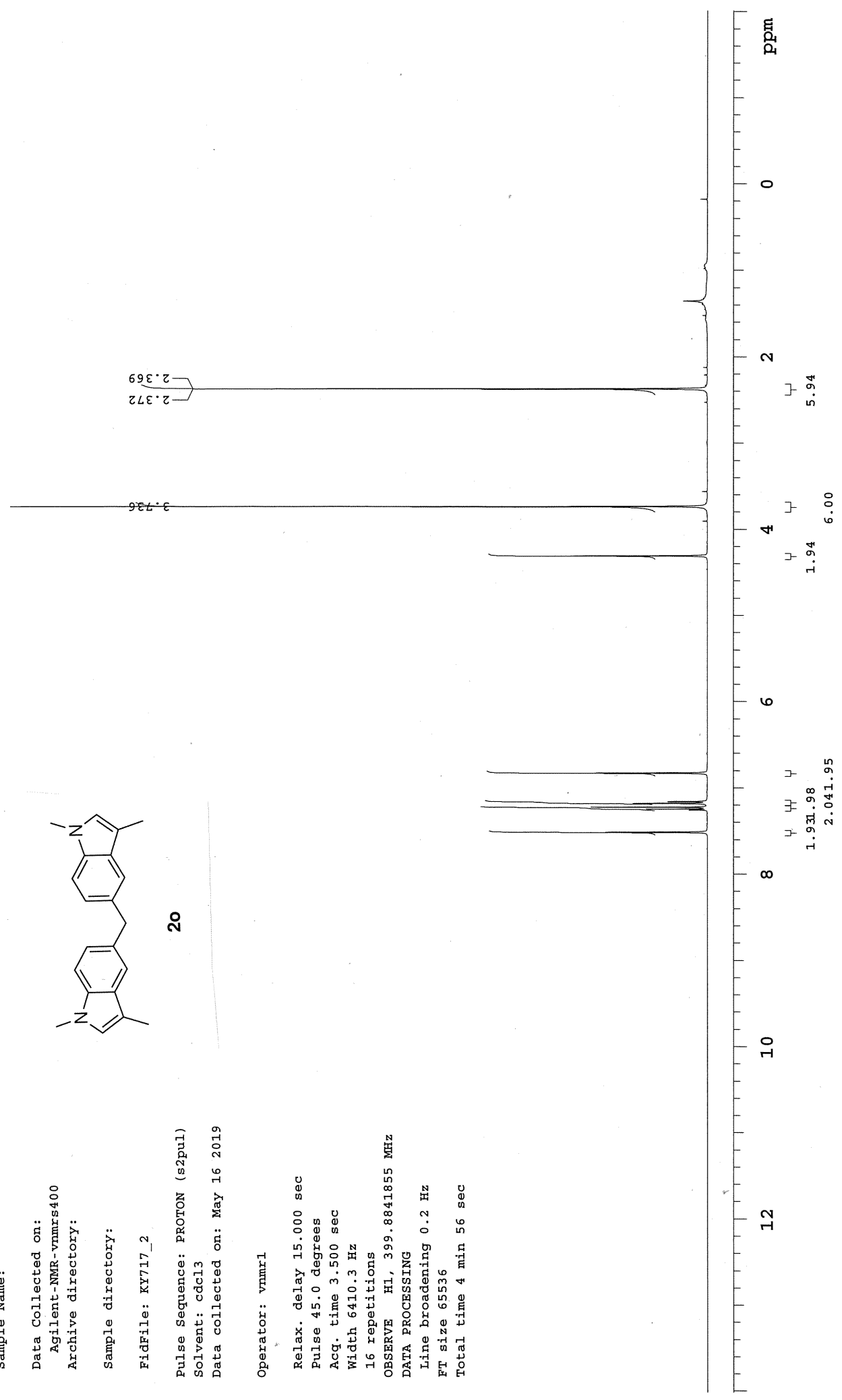

Figure S87. ${ }^{1} \mathrm{H}$ NMR Spectrum of $20\left(400 \mathrm{MHz}, \mathrm{CDCl}_{3}\right)$ 


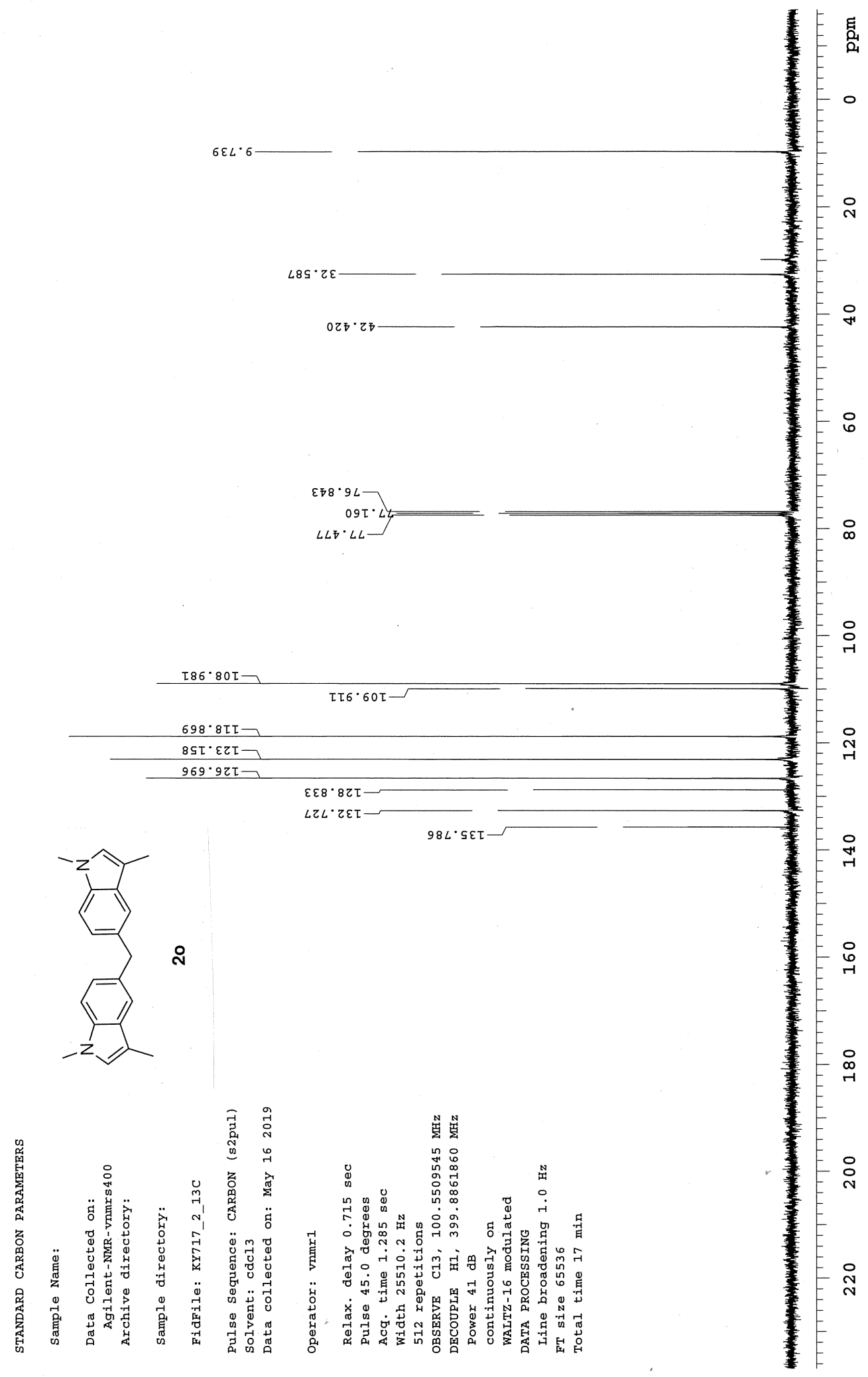

Figure S88. ${ }^{13} \mathrm{C}$ NMR Spectrum of $20\left(101 \mathrm{MHz}, \mathrm{CDCl}_{3}\right)$ 


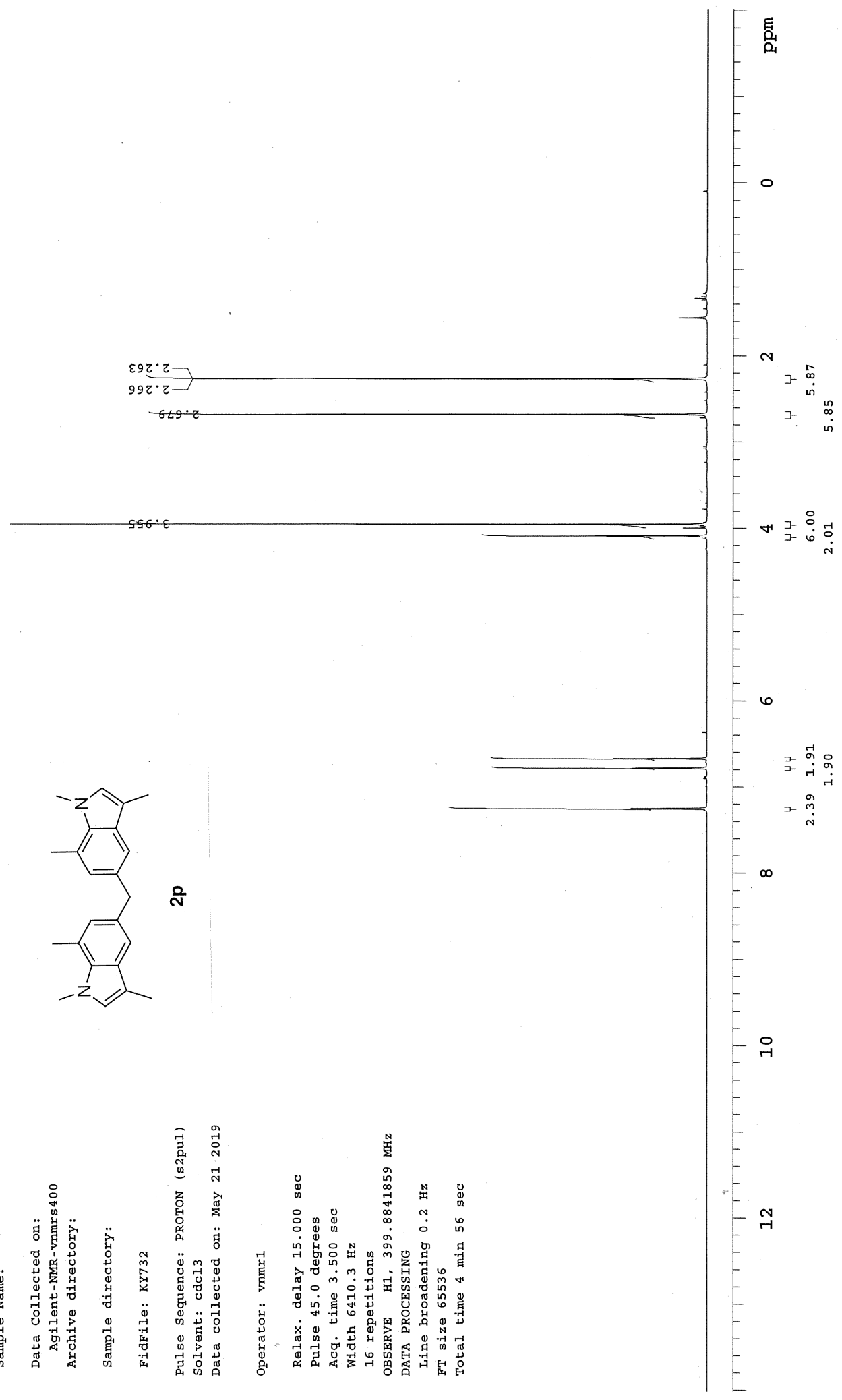

Figure S89. ${ }^{1} \mathrm{H}$ NMR Spectrum of $\mathbf{2 p}\left(400 \mathrm{MHz}, \mathrm{CDCl}_{3}\right)$ 


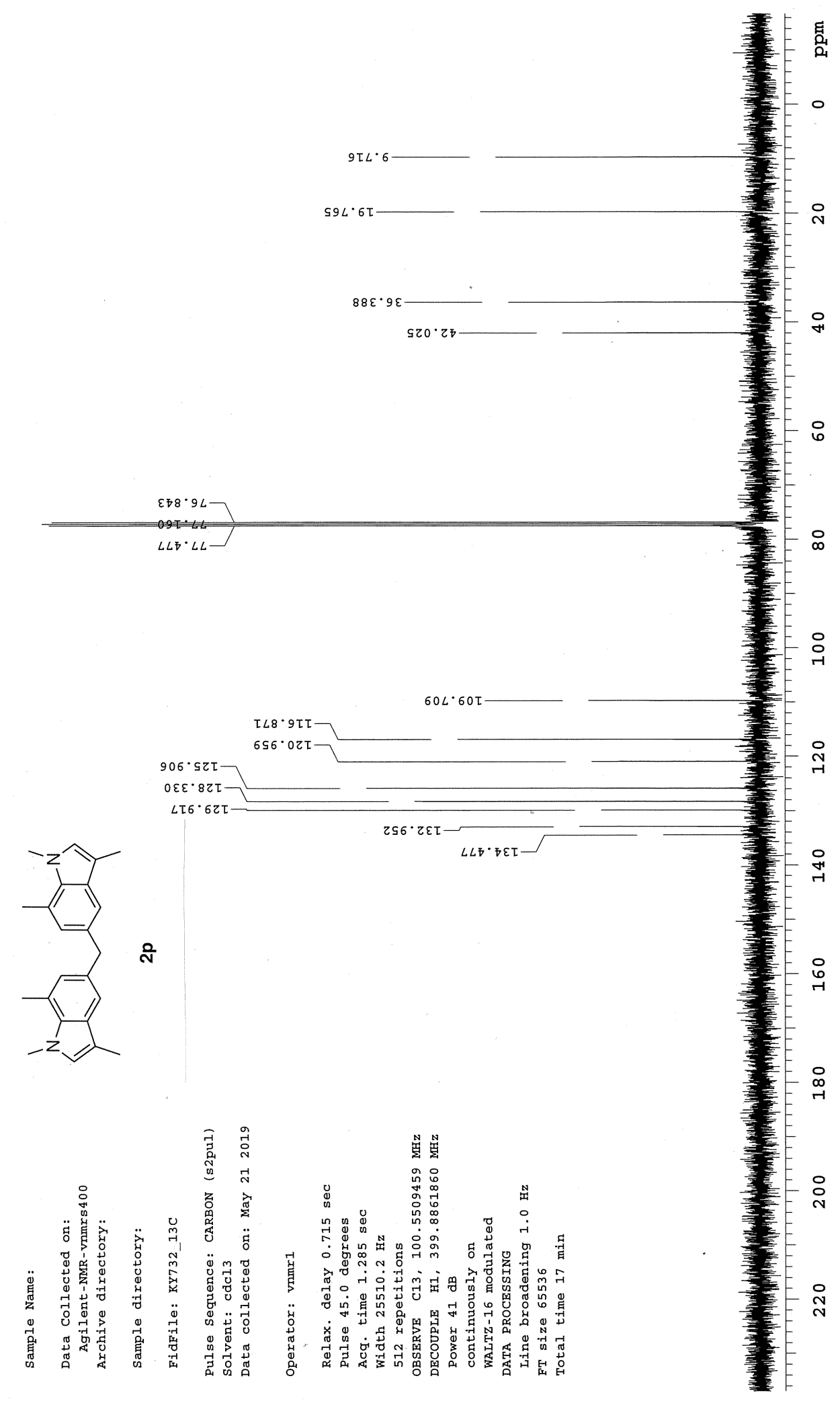

Figure S90. ${ }^{13} \mathrm{C}$ NMR Spectrum of $\mathbf{2 p}\left(101 \mathrm{MHz}, \mathrm{CDCl}_{3}\right)$ 


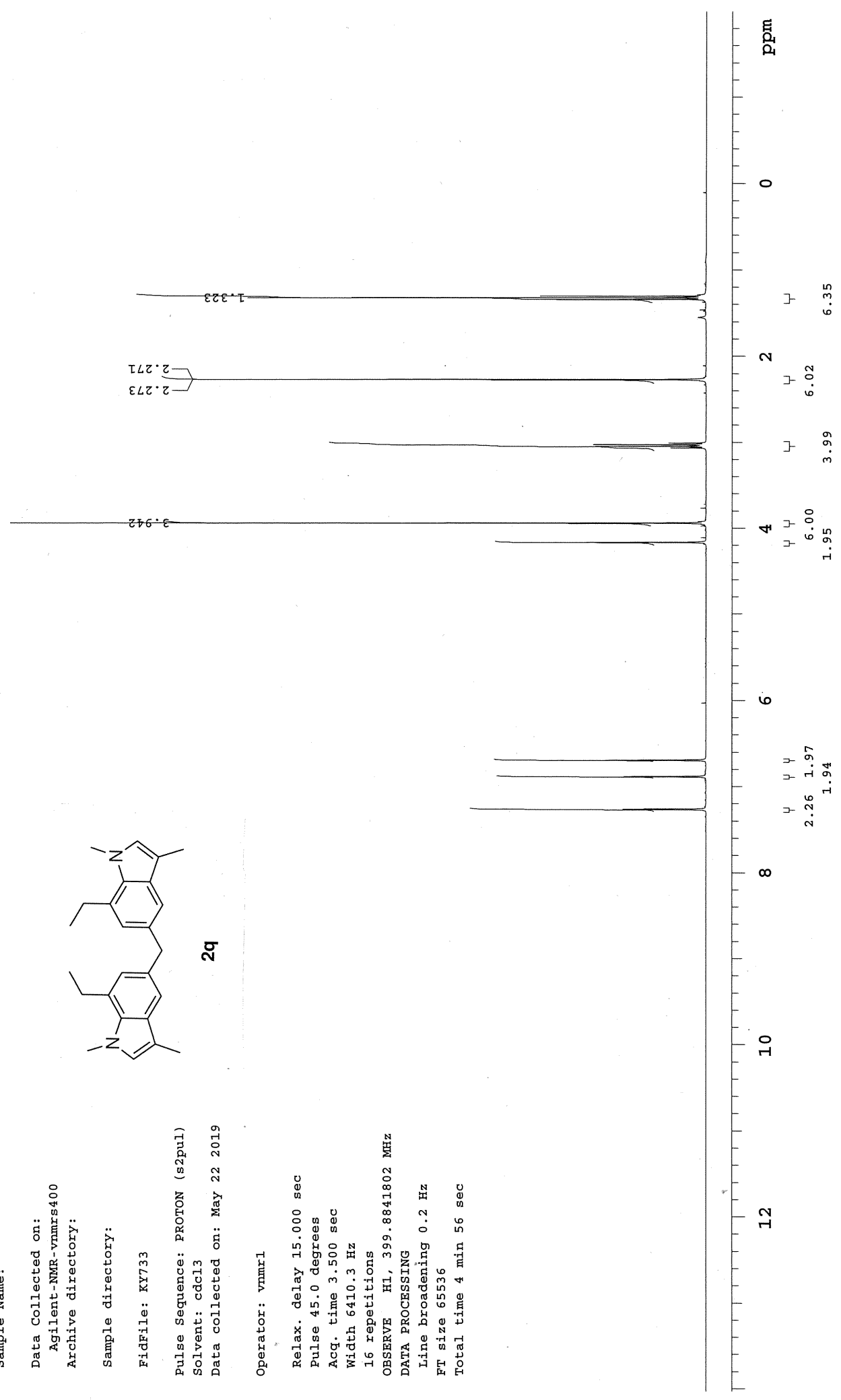

Figure S91. ${ }^{1} \mathrm{H}$ NMR Spectrum of $\mathbf{2 q}\left(400 \mathrm{MHz}, \mathrm{CDCl}_{3}\right)$ 


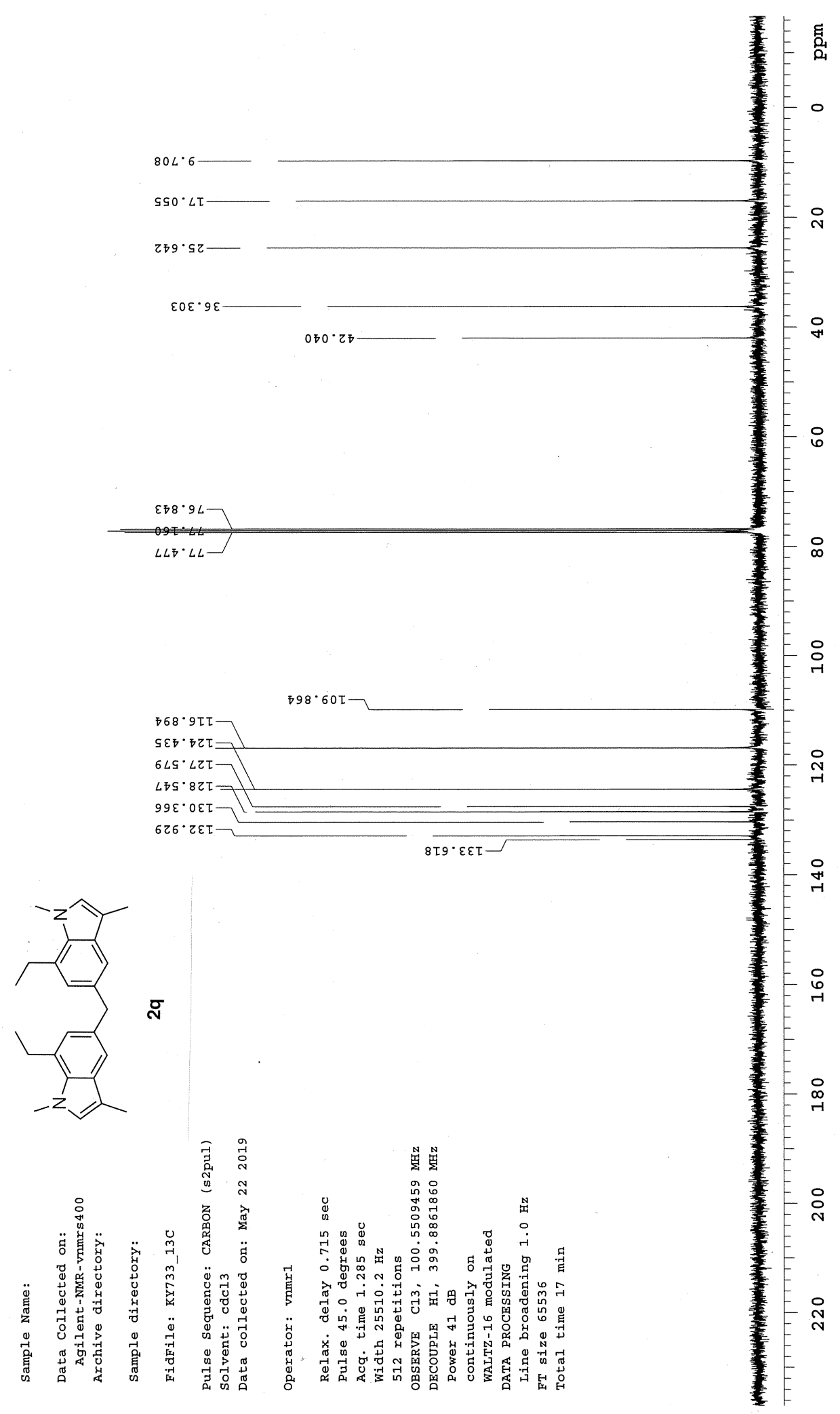

Figure S92. ${ }^{13} \mathrm{C}$ NMR Spectrum of $\mathbf{2 q}\left(101 \mathrm{MHz}, \mathrm{CDCl}_{3}\right)$ 


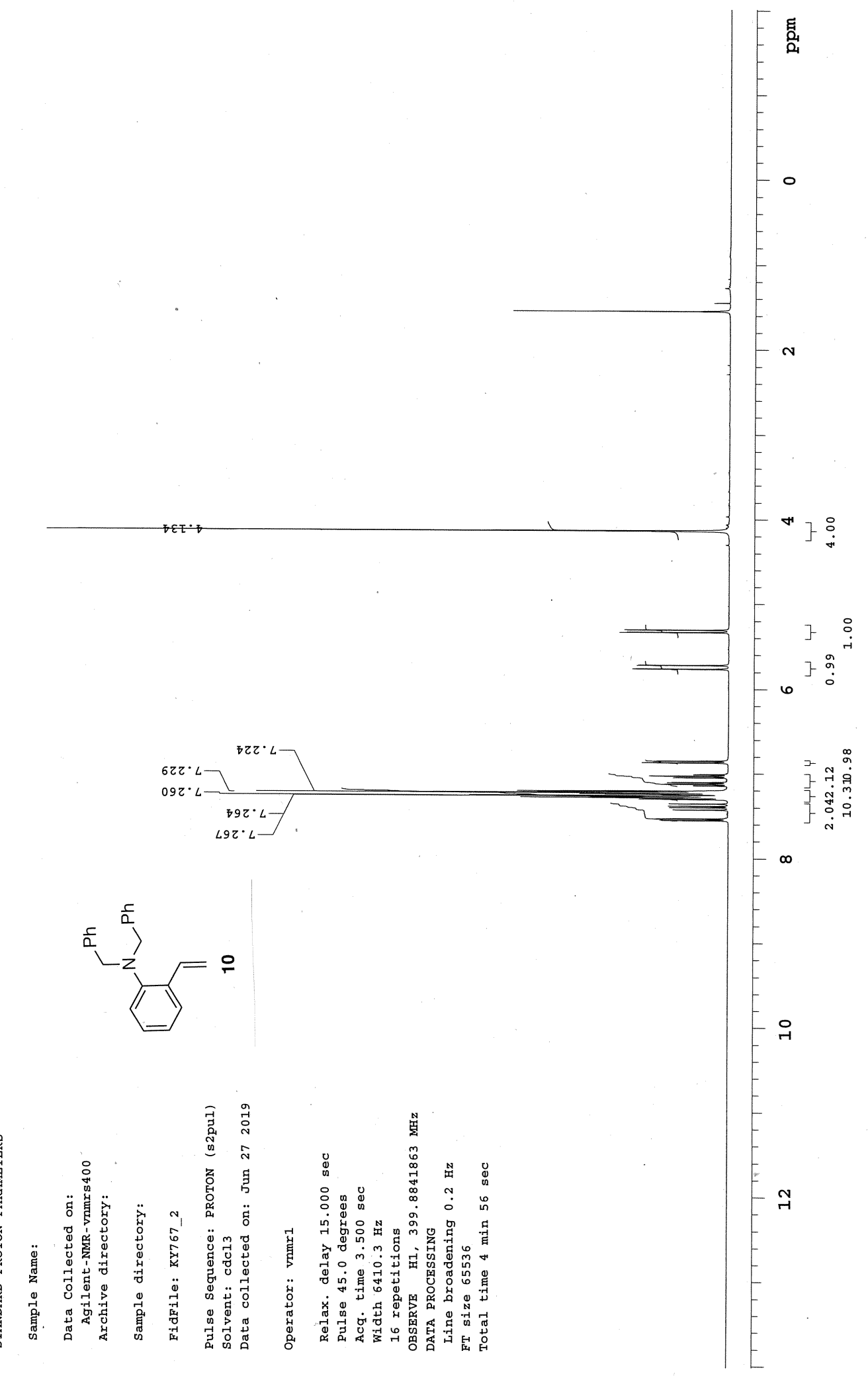

Figure S93. ${ }^{1} \mathrm{H}$ NMR Spectrum of $\mathbf{1 0}\left(400 \mathrm{MHz}, \mathrm{CDCl}_{3}\right)$ 


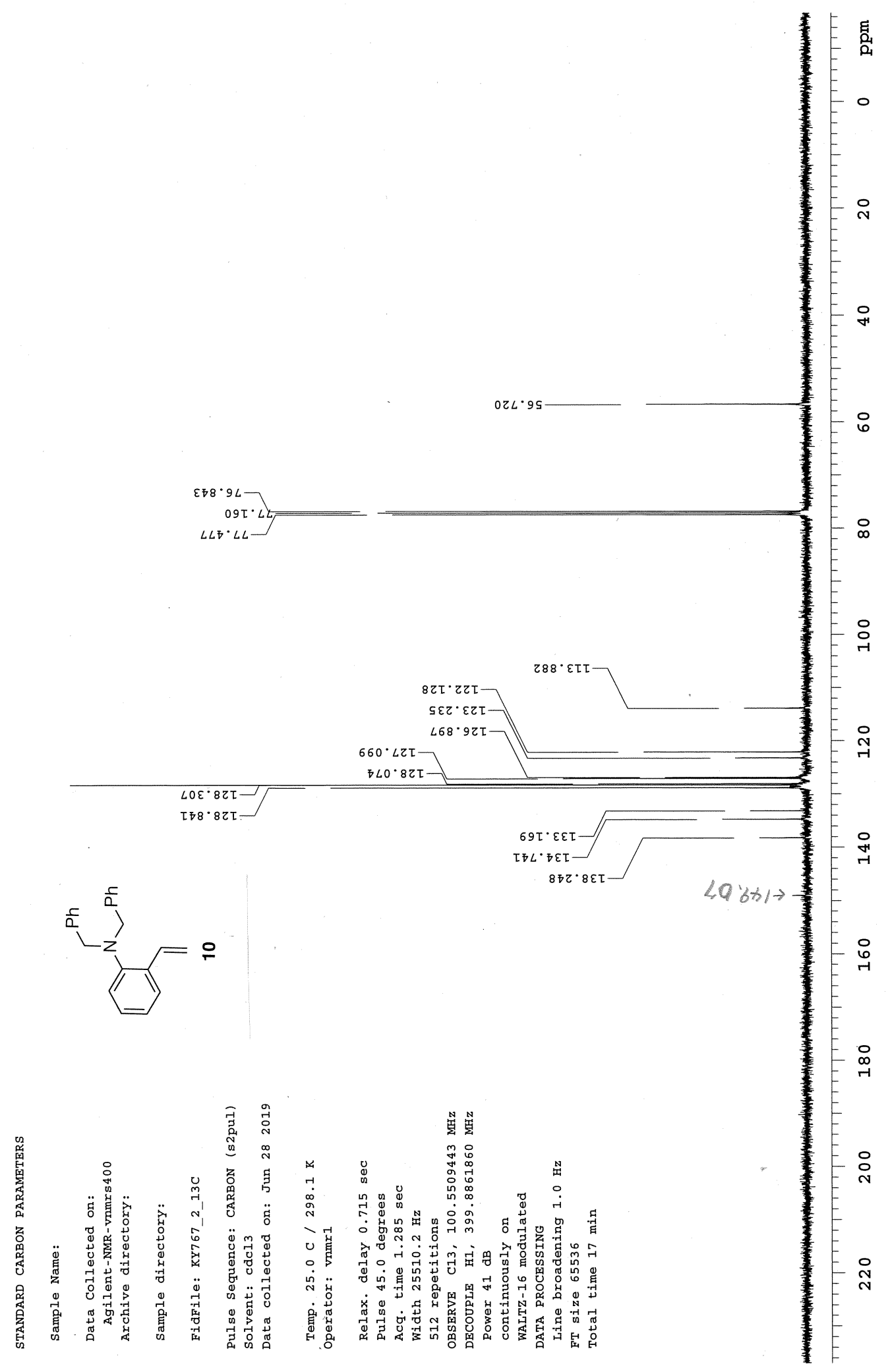

Figure S94. ${ }^{13} \mathrm{C}$ NMR Spectrum of $10\left(101 \mathrm{MHz}, \mathrm{CDCl}_{3}\right)$ 


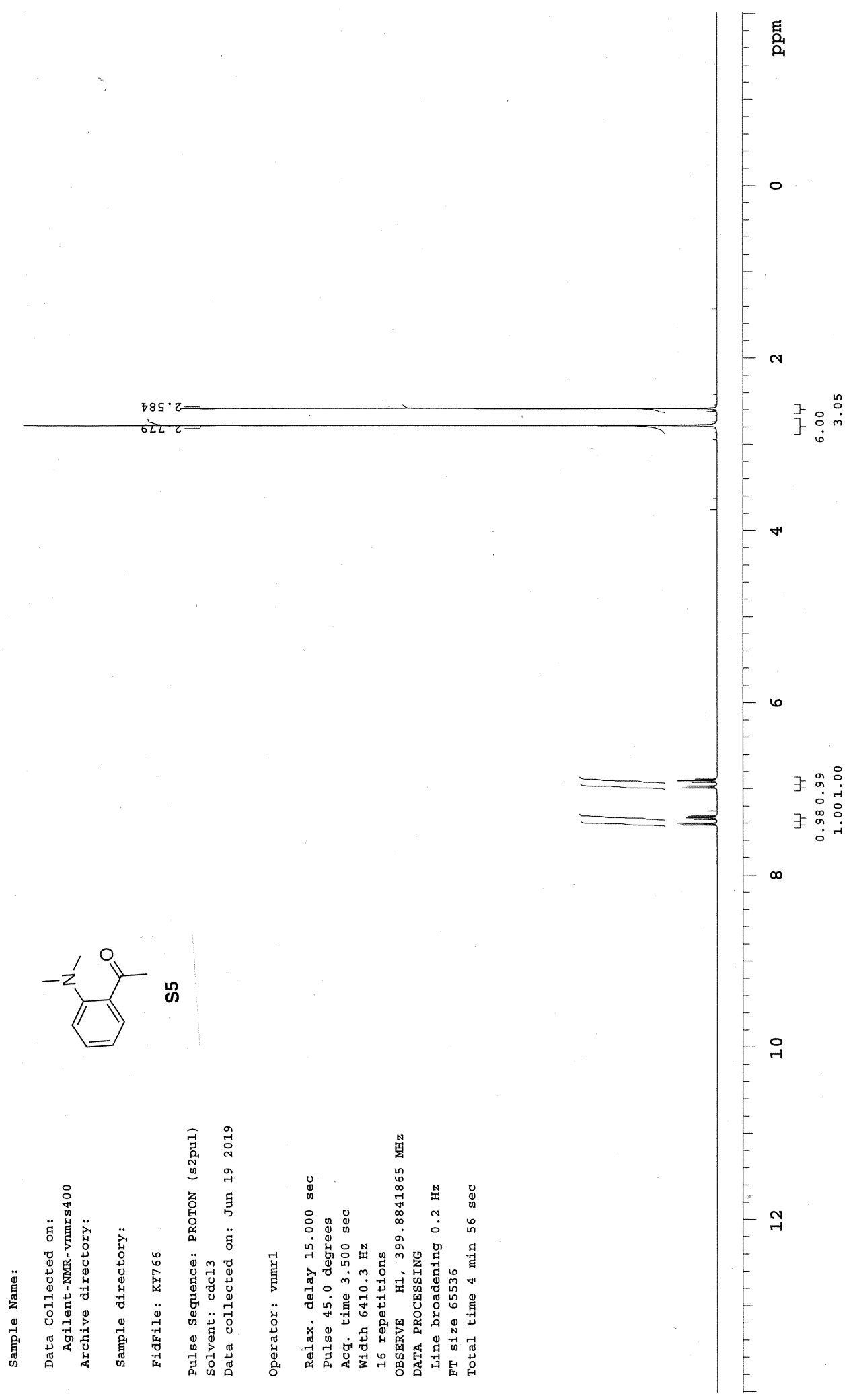

Figure S95. ${ }^{1} \mathrm{H}$ NMR Spectrum of S5 $\left(400 \mathrm{MHz}, \mathrm{CDCl}_{3}\right)$ 


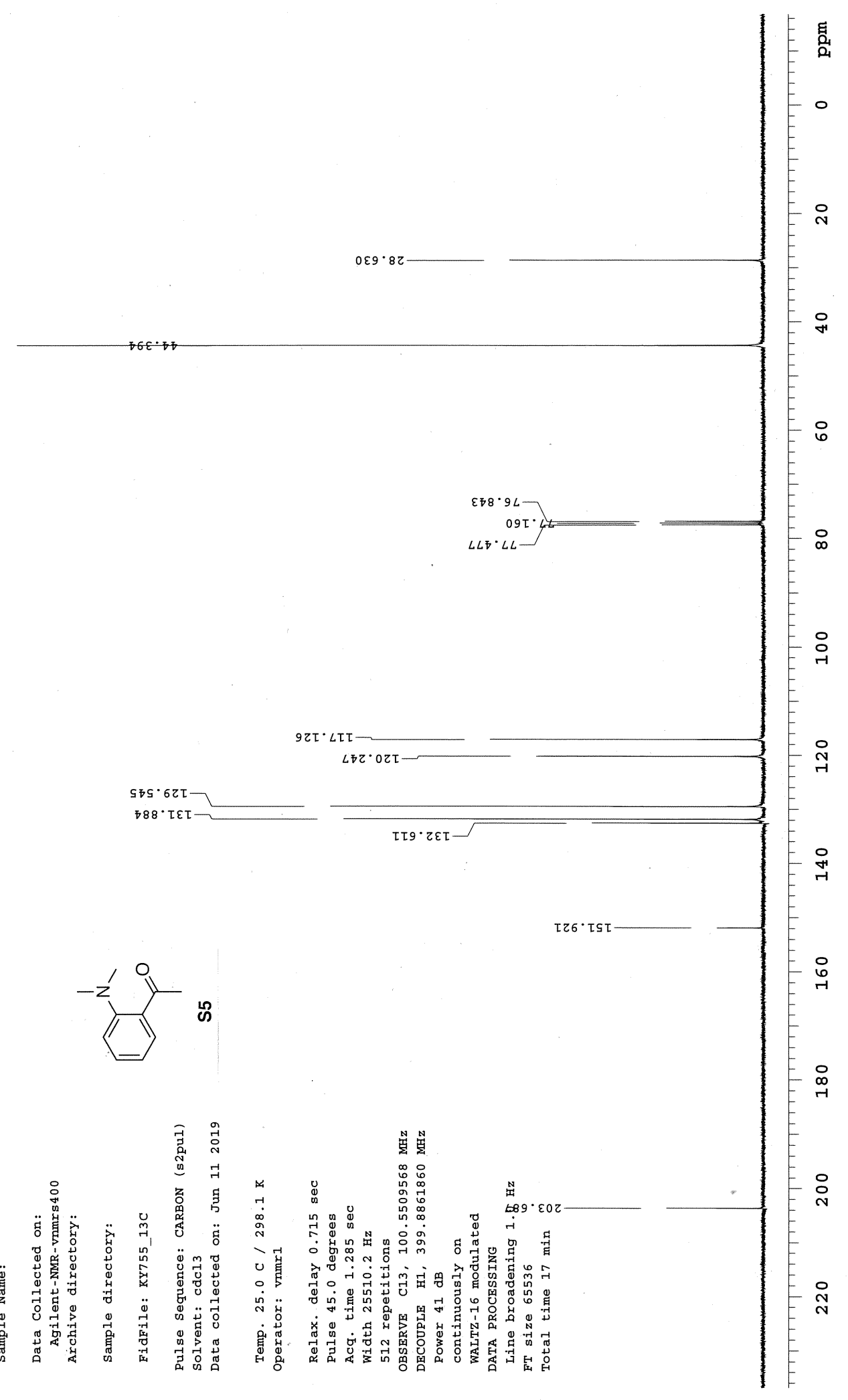

Figure S96. ${ }^{13} \mathrm{C}$ NMR Spectrum of $\mathbf{S 5}\left(101 \mathrm{MHz}, \mathrm{CDCl}_{3}\right)$ 


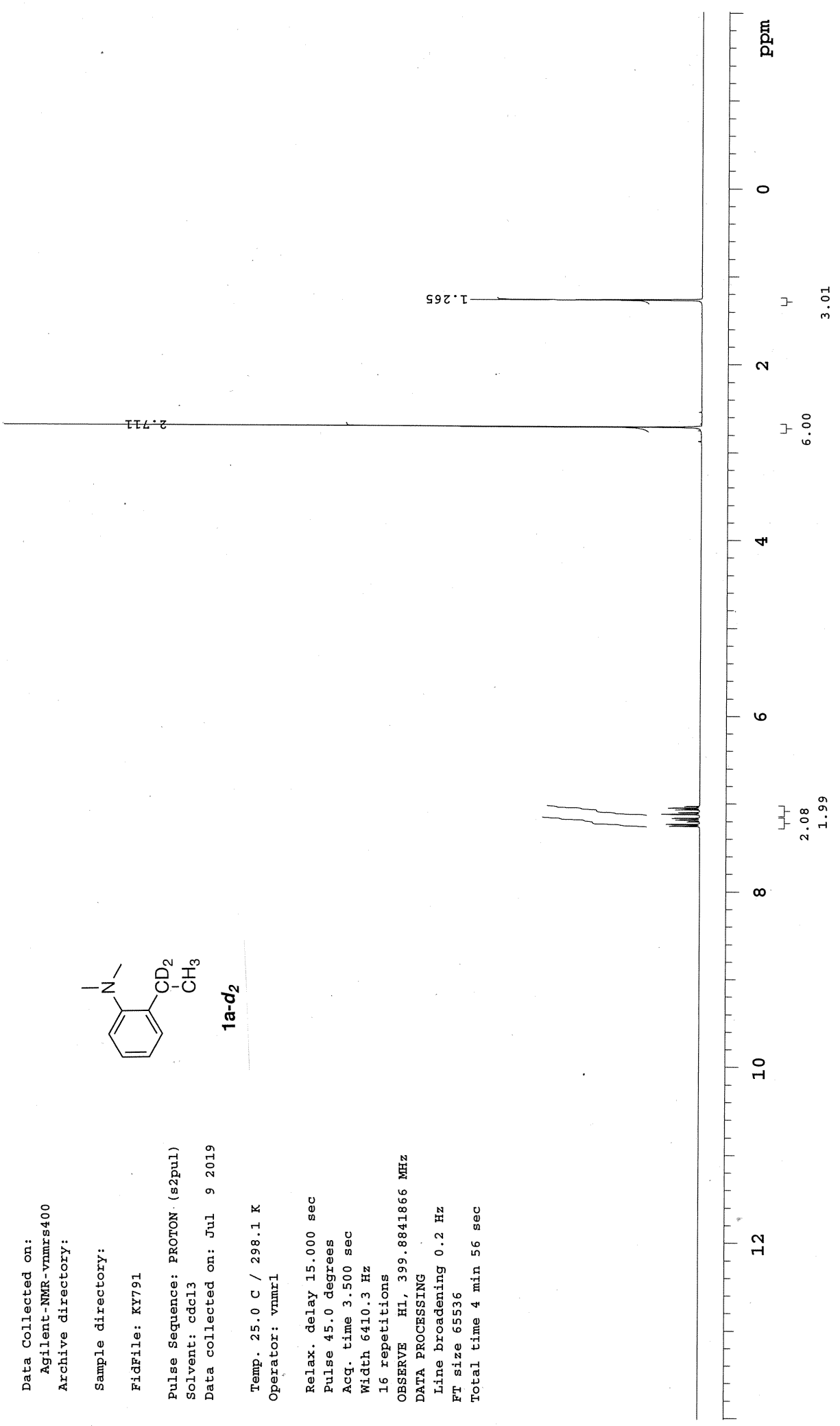

Figure S97. ${ }^{1} \mathrm{H} \mathrm{NMR}$ Spectrum of $\mathbf{1 a - d _ { 2 }}\left(400 \mathrm{MHz}, \mathrm{CDCl}_{3}\right)$ 


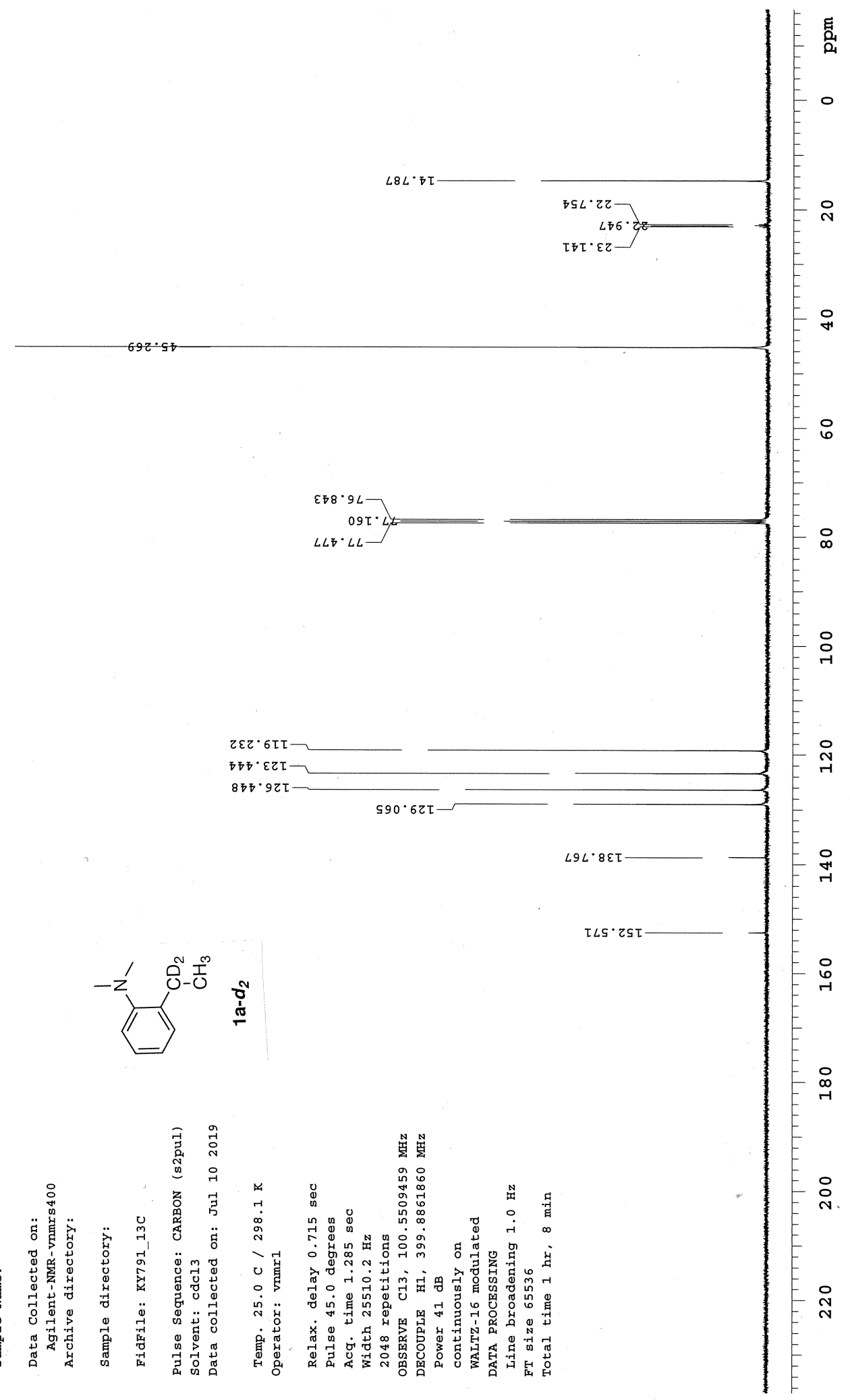

Figure S98. ${ }^{13} \mathrm{C}$ NMR Spectrum of $\mathbf{1 a - d _ { 2 }}\left(101 \mathrm{MHz}, \mathrm{CDCl}_{3}\right)$ 


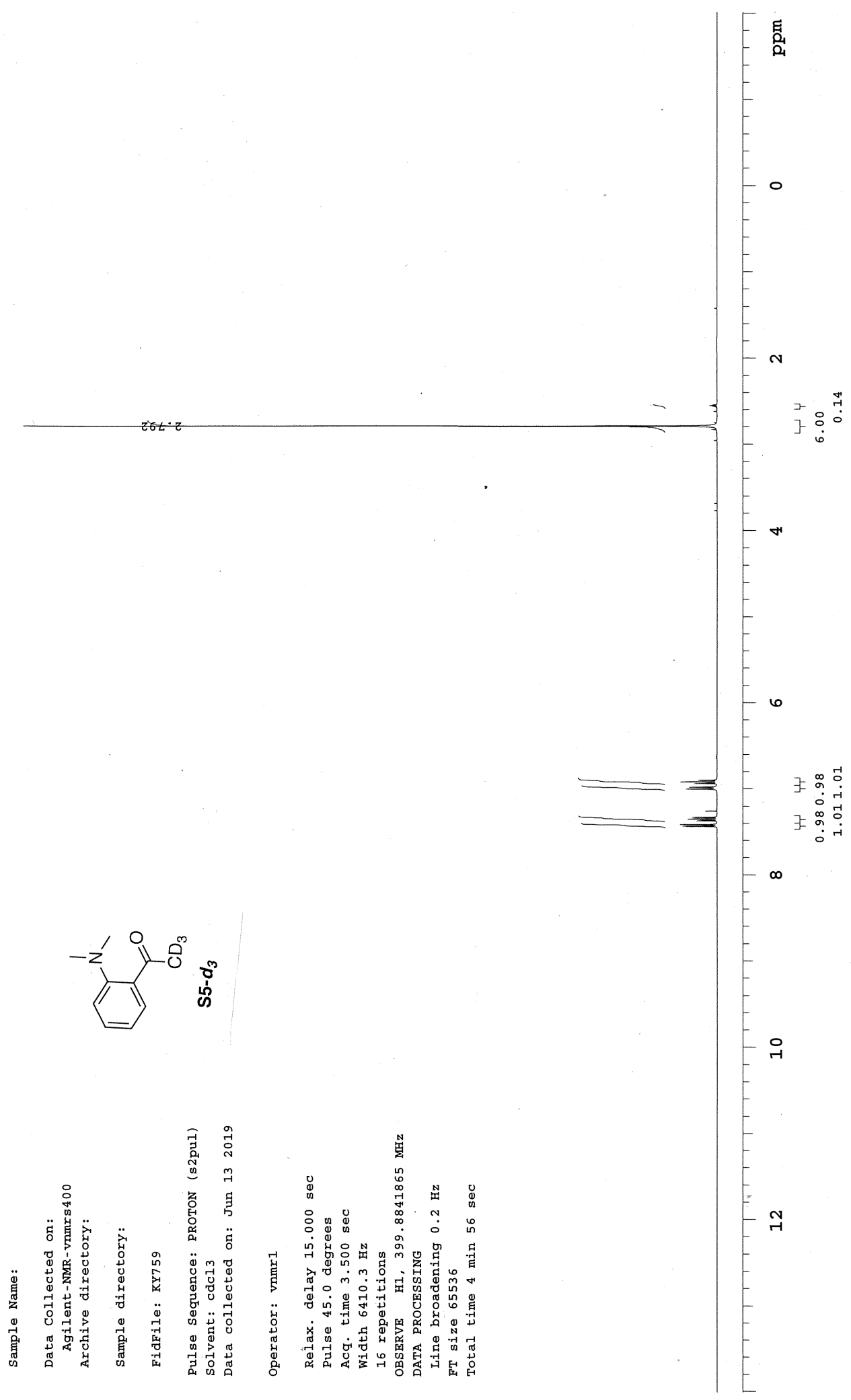

Figure S99. ${ }^{1} \mathrm{H}$ NMR Spectrum of $\mathbf{S 5 - d _ { 3 }}\left(400 \mathrm{MHz}, \mathrm{CDCl}_{3}\right)$ 


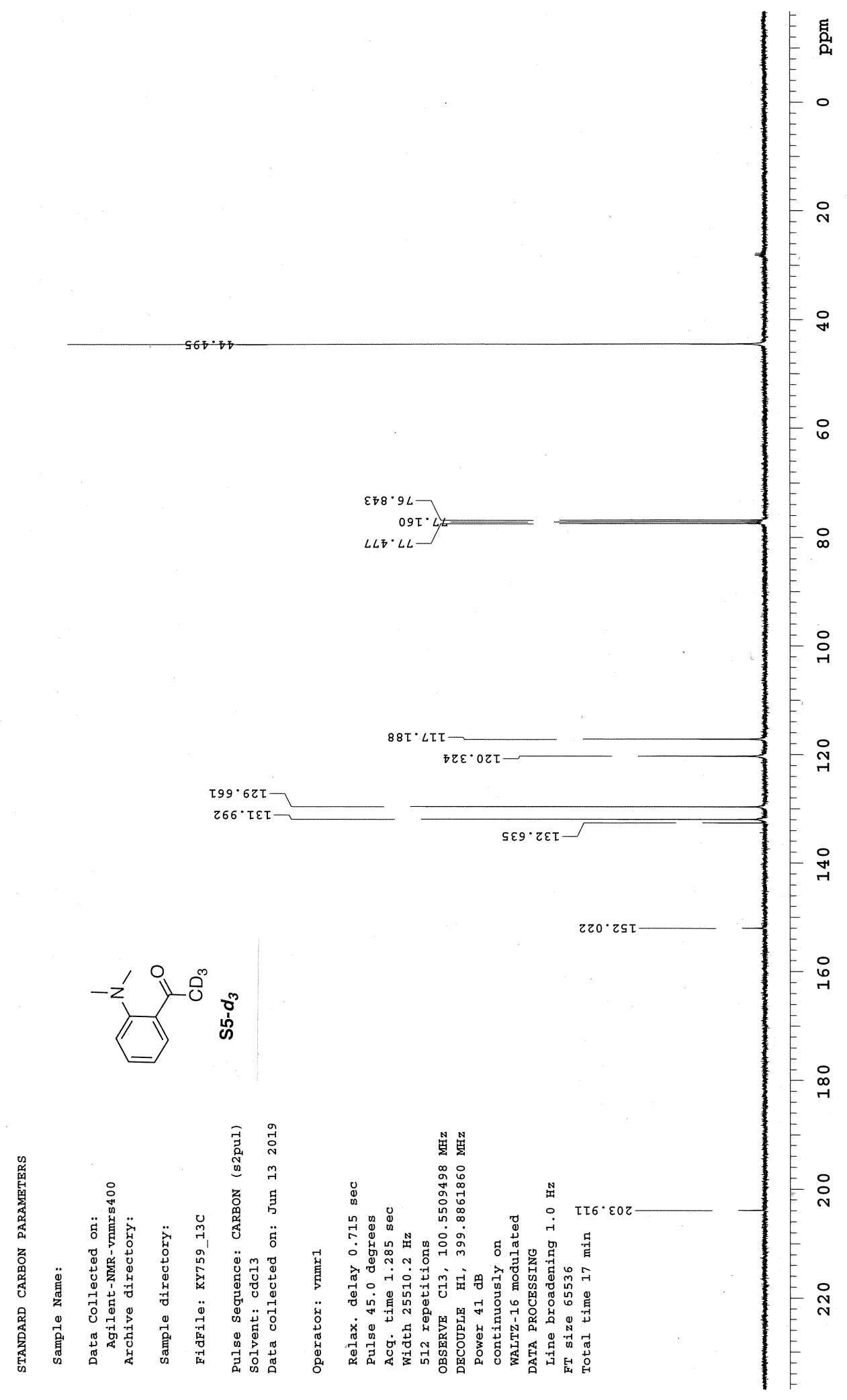

Figure S100. ${ }^{13} \mathrm{C}$ NMR Spectrum of $\mathbf{S 5 - d _ { 3 }}\left(101 \mathrm{MHz}, \mathrm{CDCl}_{3}\right)$ 


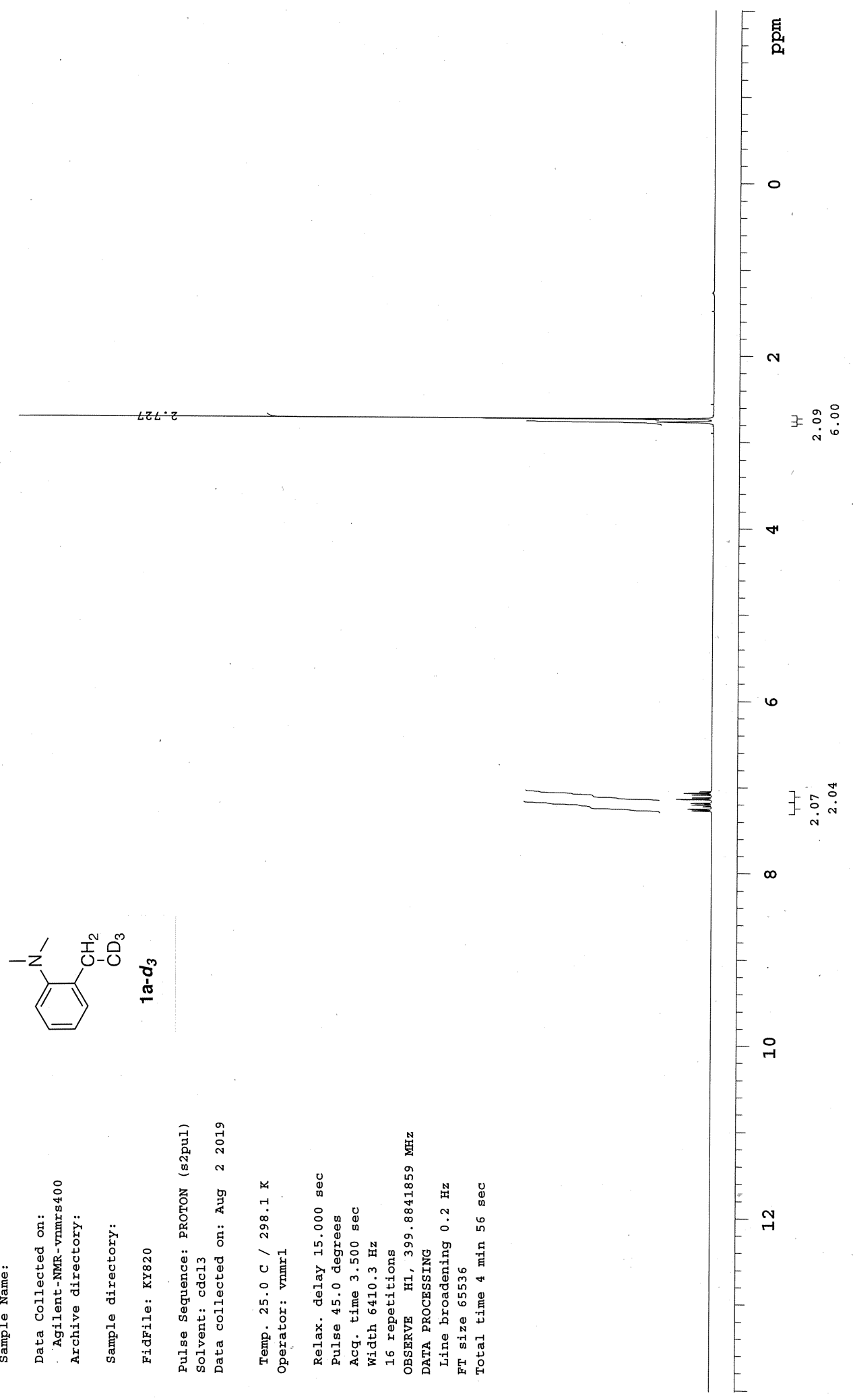

Figure S101. ${ }^{1} \mathrm{H}$ NMR Spectrum of $\mathbf{1 a -} \boldsymbol{d}_{3}\left(400 \mathrm{MHz}, \mathrm{CDCl}_{3}\right)$ 


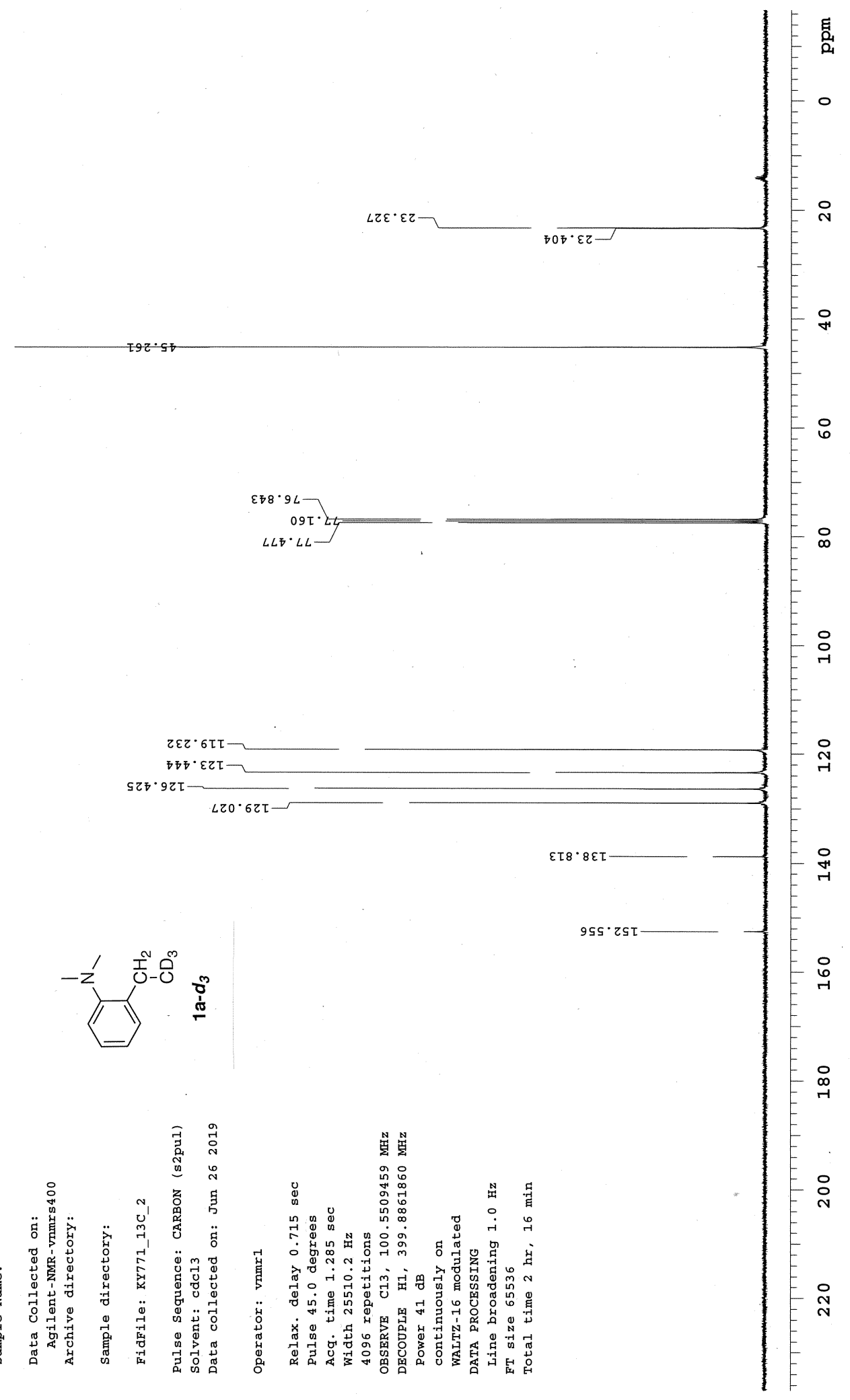

Figure S102. ${ }^{13} \mathrm{C}$ NMR Spectrum of $\mathbf{1 a -} \boldsymbol{d}_{3}\left(101 \mathrm{MHz}, \mathrm{CDCl}_{3}\right)$ 


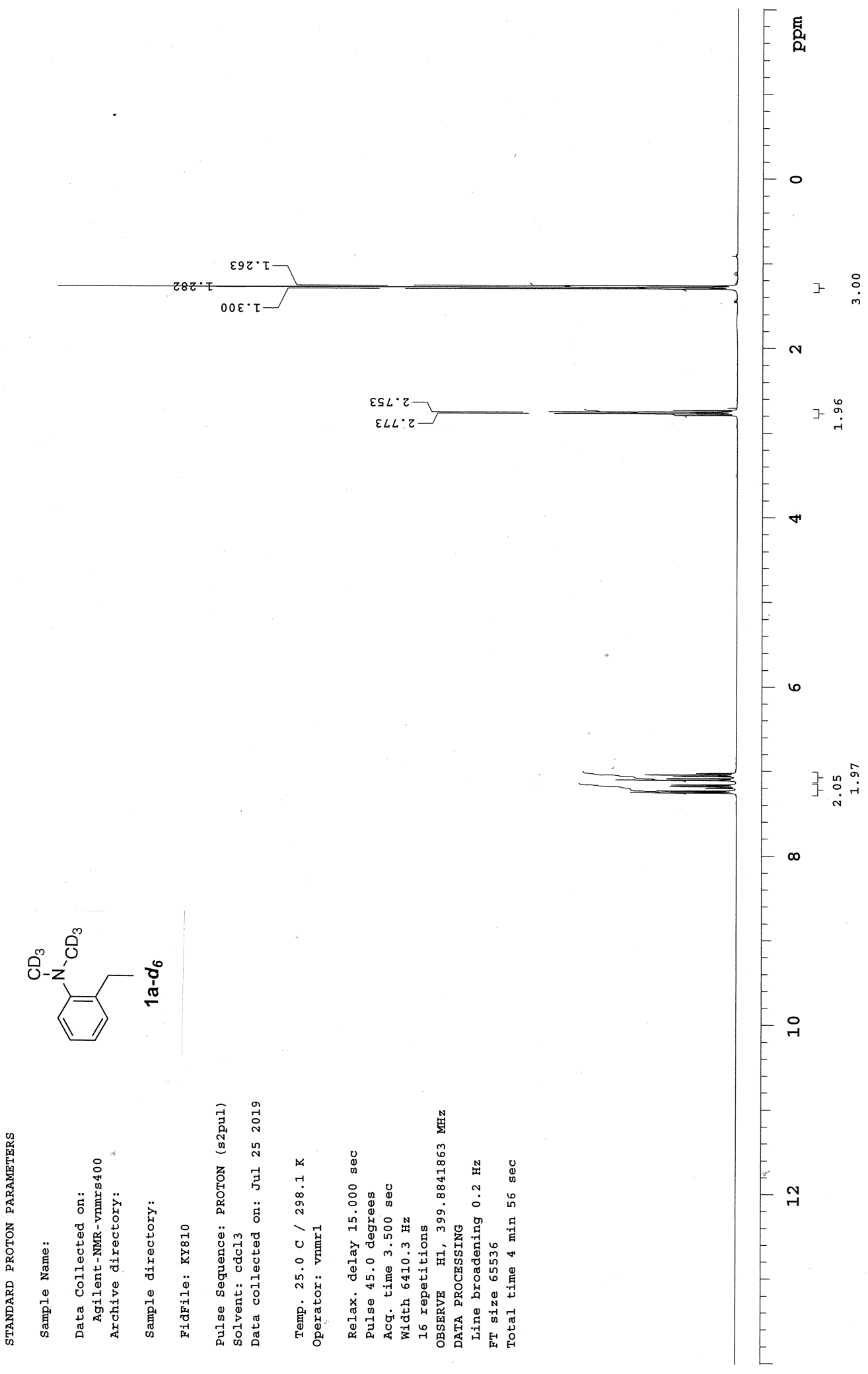

Figure S103. ${ }^{1} \mathrm{H}$ NMR Spectrum of $\mathbf{1 a - d} \boldsymbol{d}_{\boldsymbol{6}}\left(400 \mathrm{MHz}, \mathrm{CDCl}_{3}\right)$ 


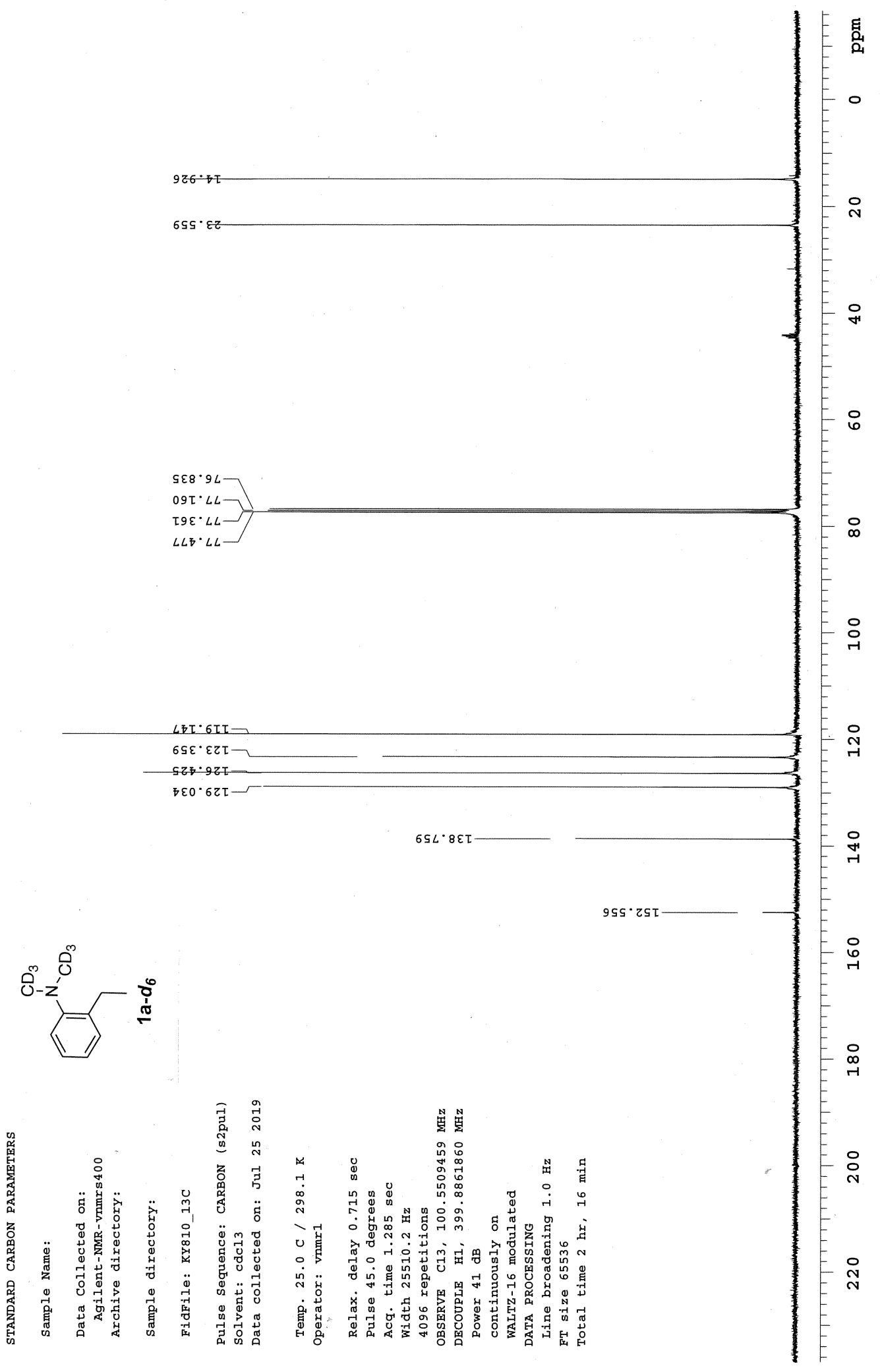

Figure S104. ${ }^{13} \mathrm{C}$ NMR Spectrum of $\mathbf{1 a - d _ { 6 }}\left(101 \mathrm{MHz}, \mathrm{CDCl}_{3}\right)$ 


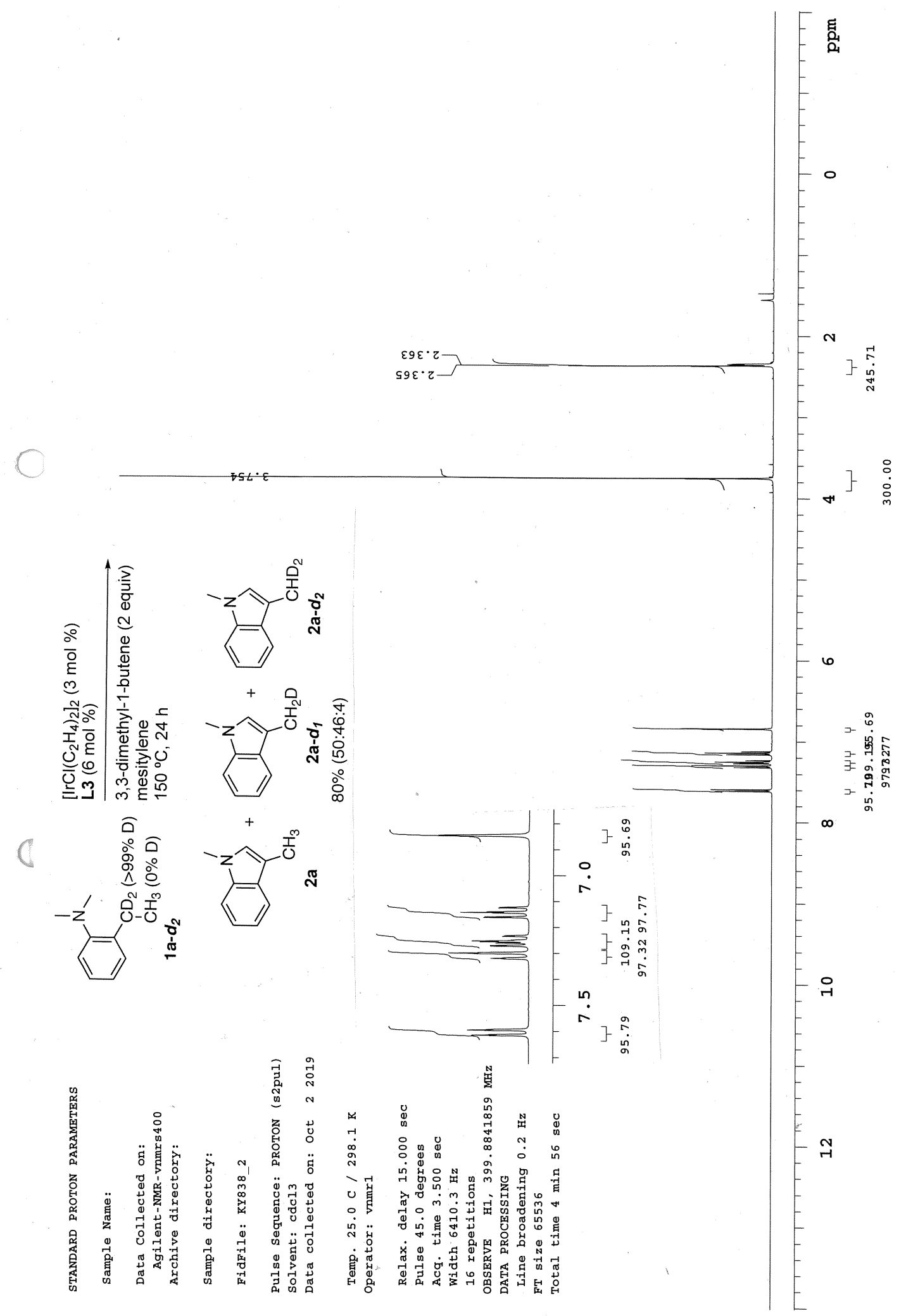

Figure S105. ${ }^{1} \mathrm{H}$ NMR Spectrum for the Reaction of $1 \mathbf{a}-\boldsymbol{d}_{2}\left(400 \mathrm{MHz}, \mathrm{CDCl}_{3}\right)$ 


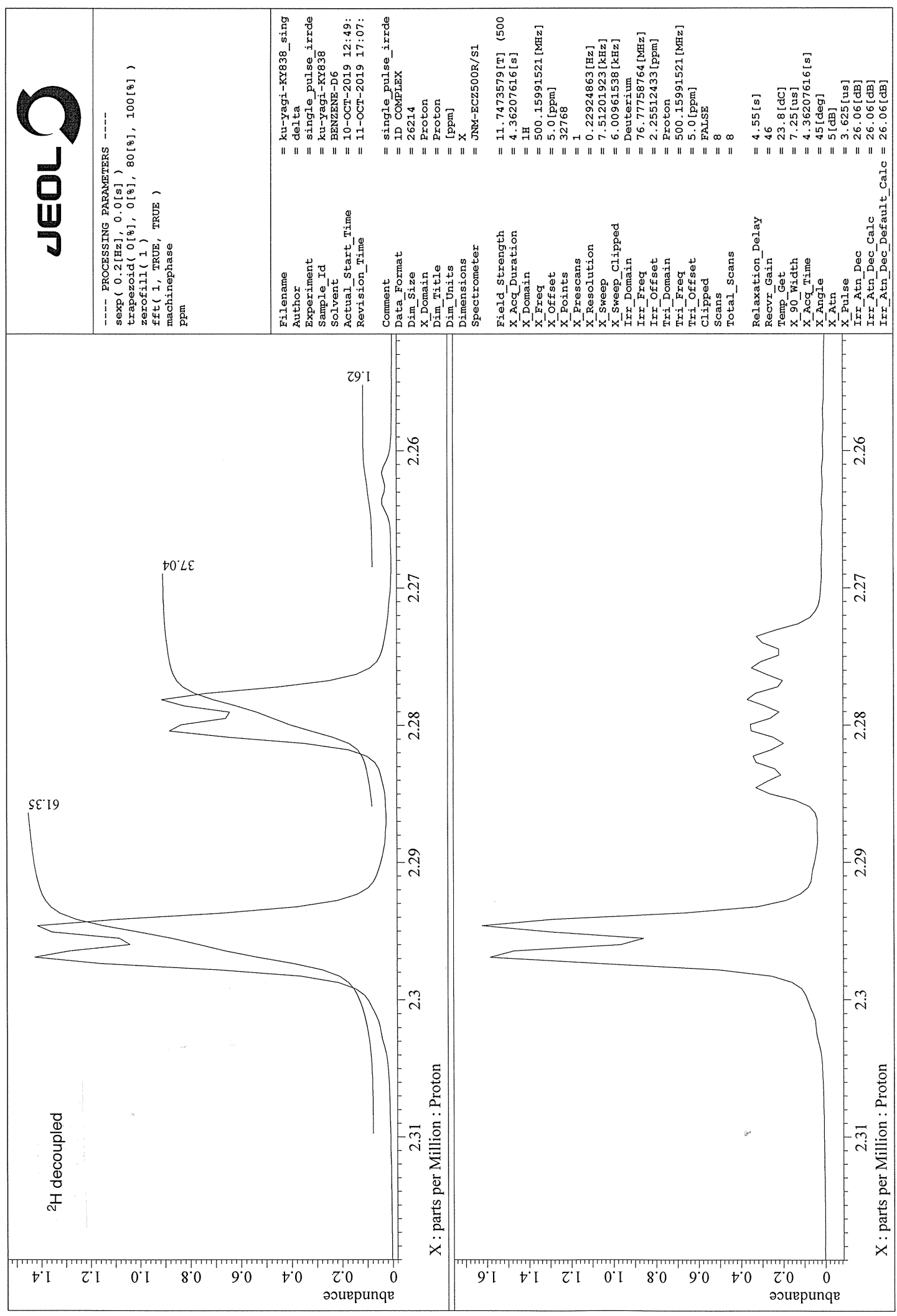

Figure S106. ${ }^{1} \mathrm{H}$ NMR Spectrum for the Reaction of $1 \mathbf{a}-\boldsymbol{d}_{2}$, Expansion $\left(500 \mathrm{MHz}, \mathrm{C}_{6} \mathrm{D}_{6}\right)$ 


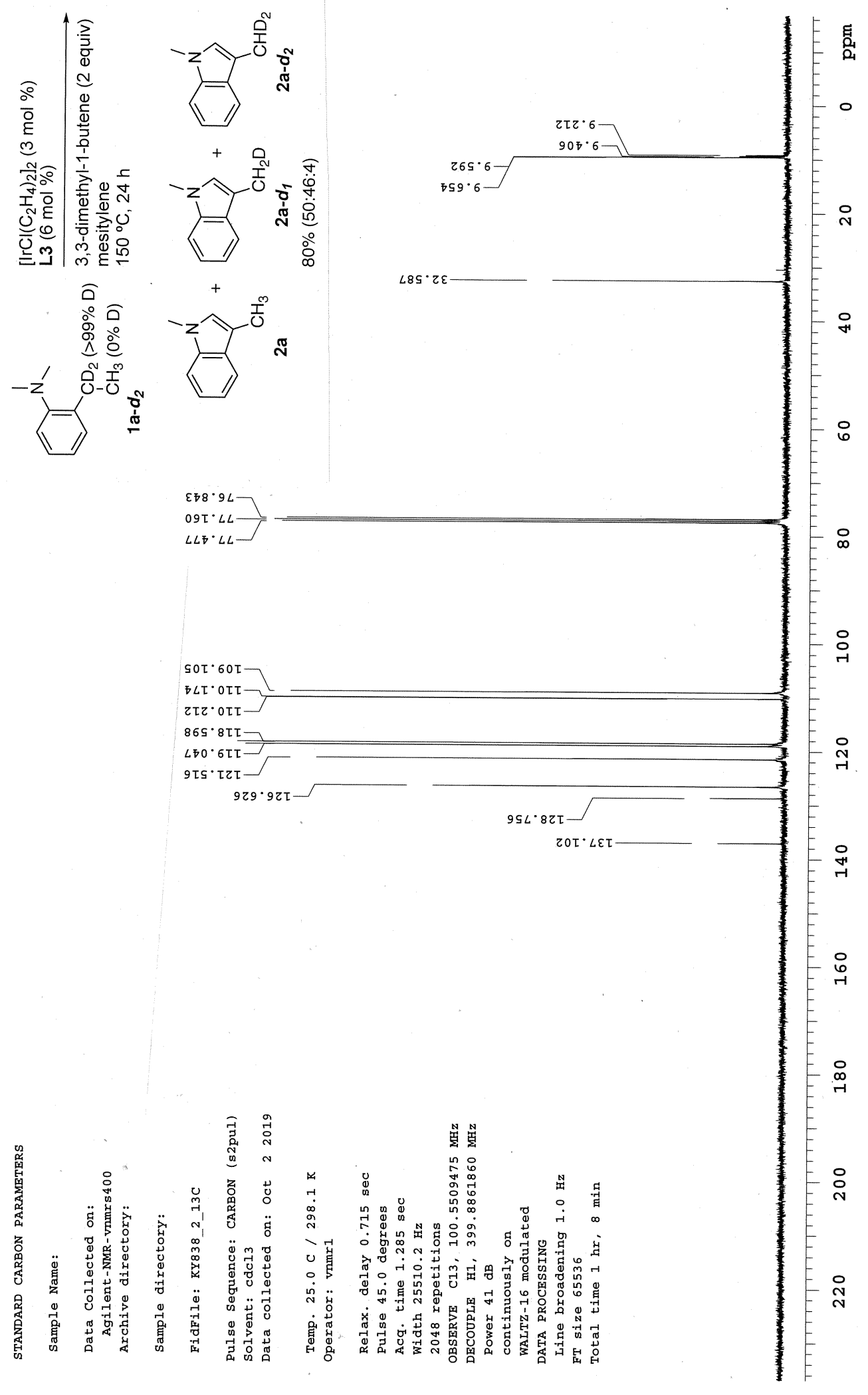

Figure S107. ${ }^{13} \mathrm{C}$ NMR Spectrum for the Reaction of $\mathbf{1 a}-\boldsymbol{d}_{2}\left(101 \mathrm{MHz}, \mathrm{CDCl}_{3}\right)$ 


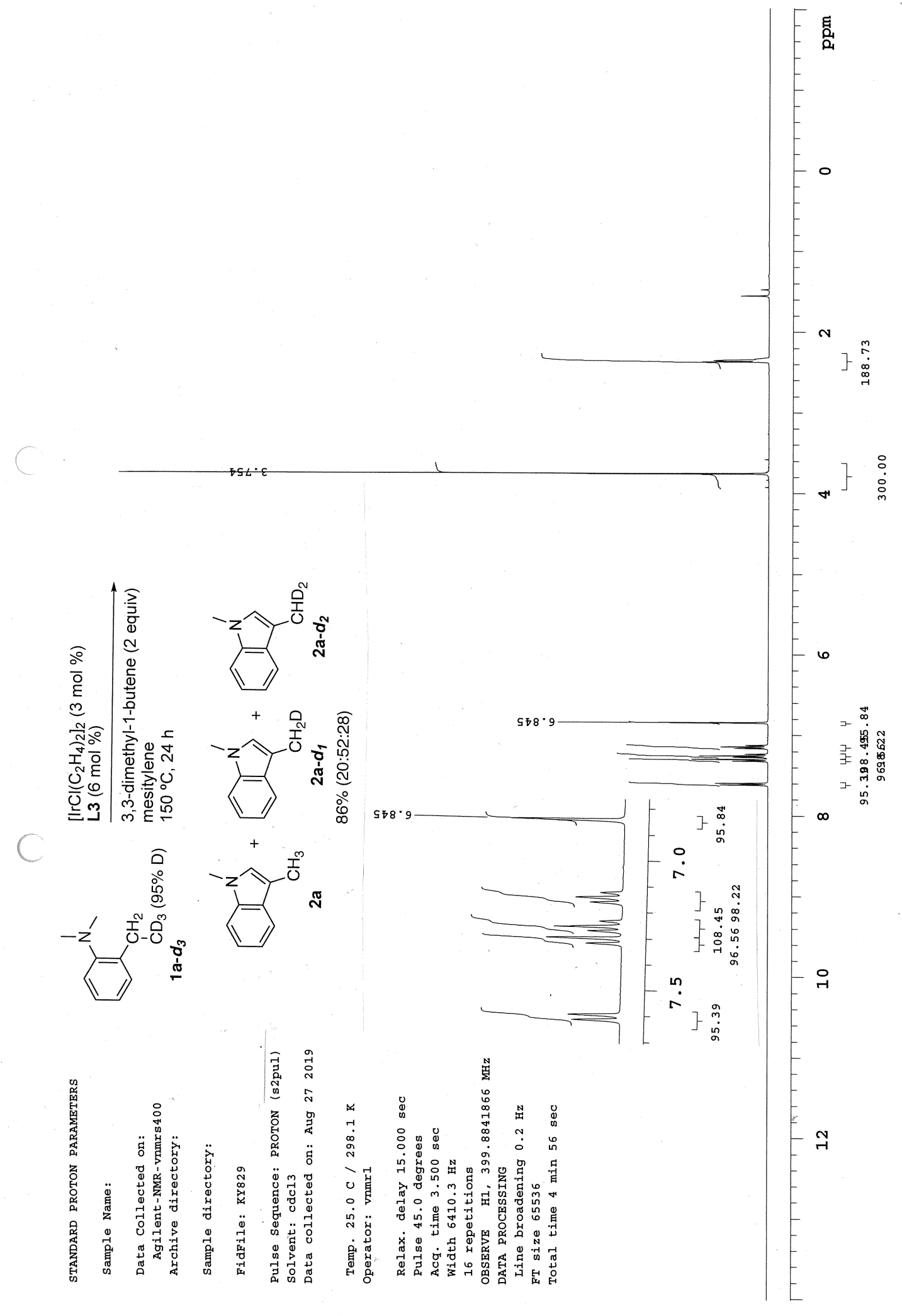

Figure S108. ${ }^{1} \mathrm{H}$ NMR Spectrum for the Reaction of $\mathbf{1 a}-\boldsymbol{d}_{3}\left(400 \mathrm{MHz}, \mathrm{CDCl}_{3}\right)$ 


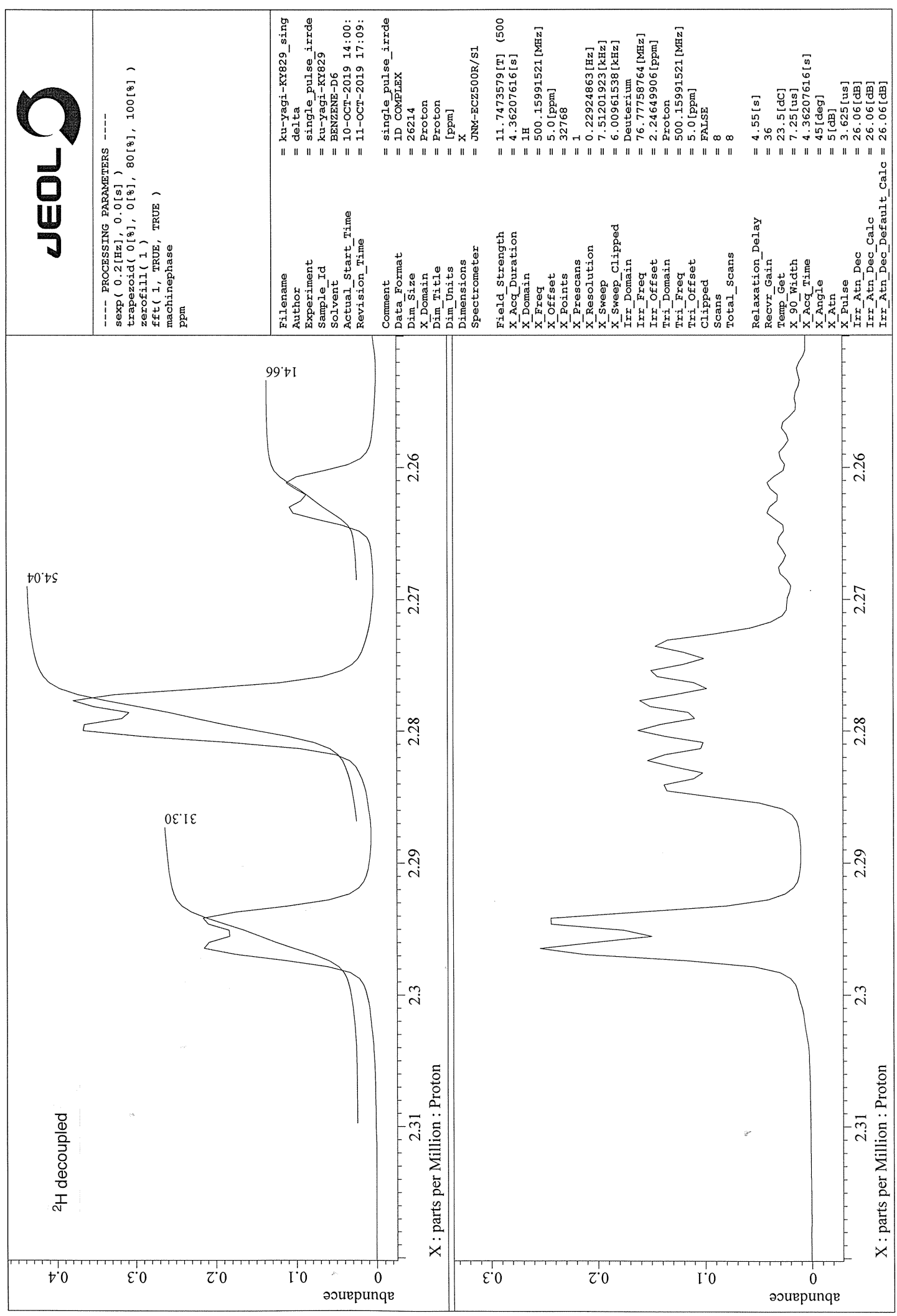

Figure S109. ${ }^{1} \mathrm{H}$ NMR Spectrum for the Reaction of $1 \mathbf{a}-\boldsymbol{d}_{3}$, Expansion $\left(500 \mathrm{MHz}, \mathrm{C}_{6} \mathrm{D}_{6}\right)$ 


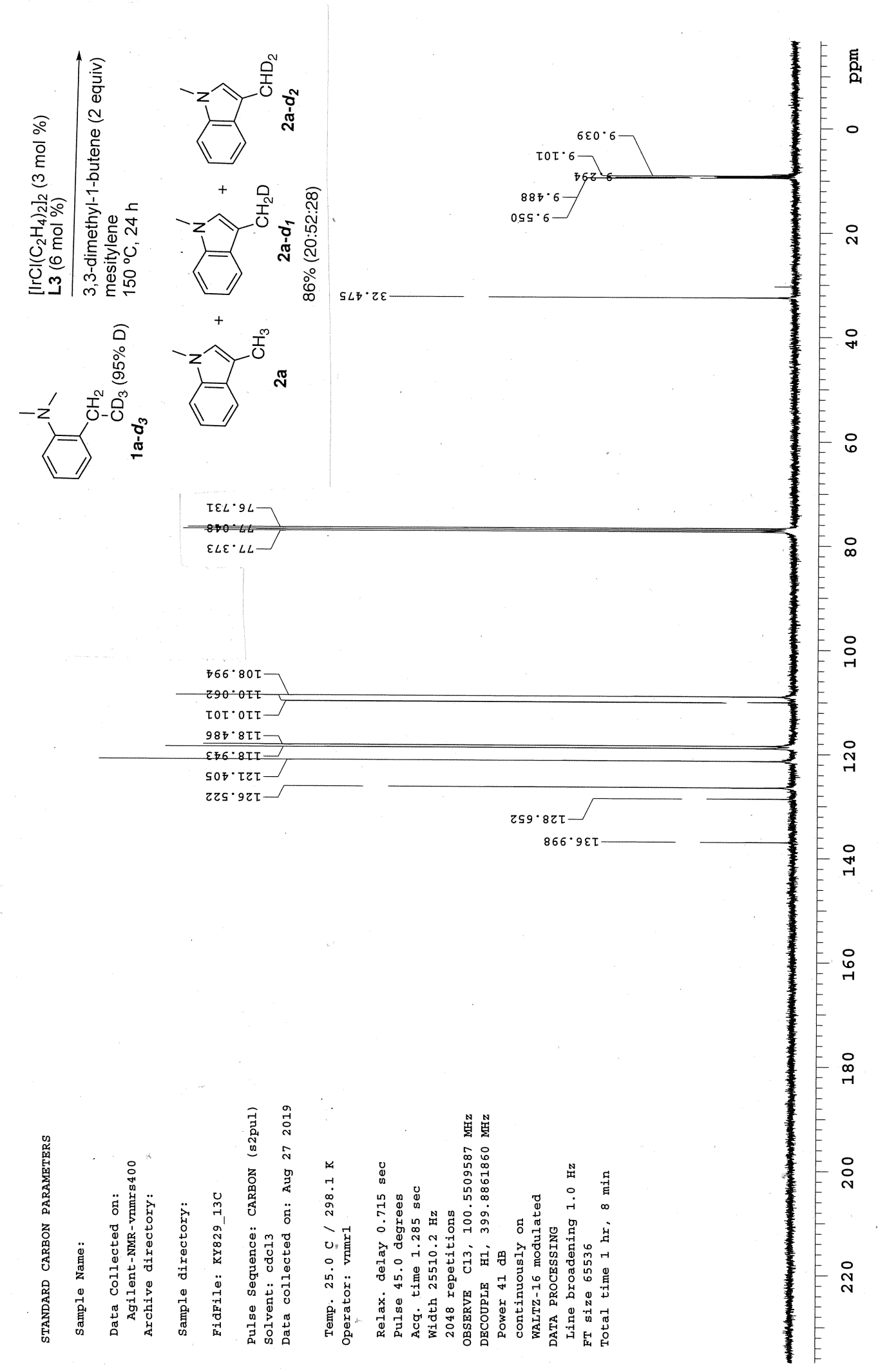

Figure S110. ${ }^{13} \mathrm{C}$ NMR Spectrum for the Reaction of $1 \mathbf{a}-\boldsymbol{d}_{3}\left(101 \mathrm{MHz}, \mathrm{CDCl}_{3}\right)$ 


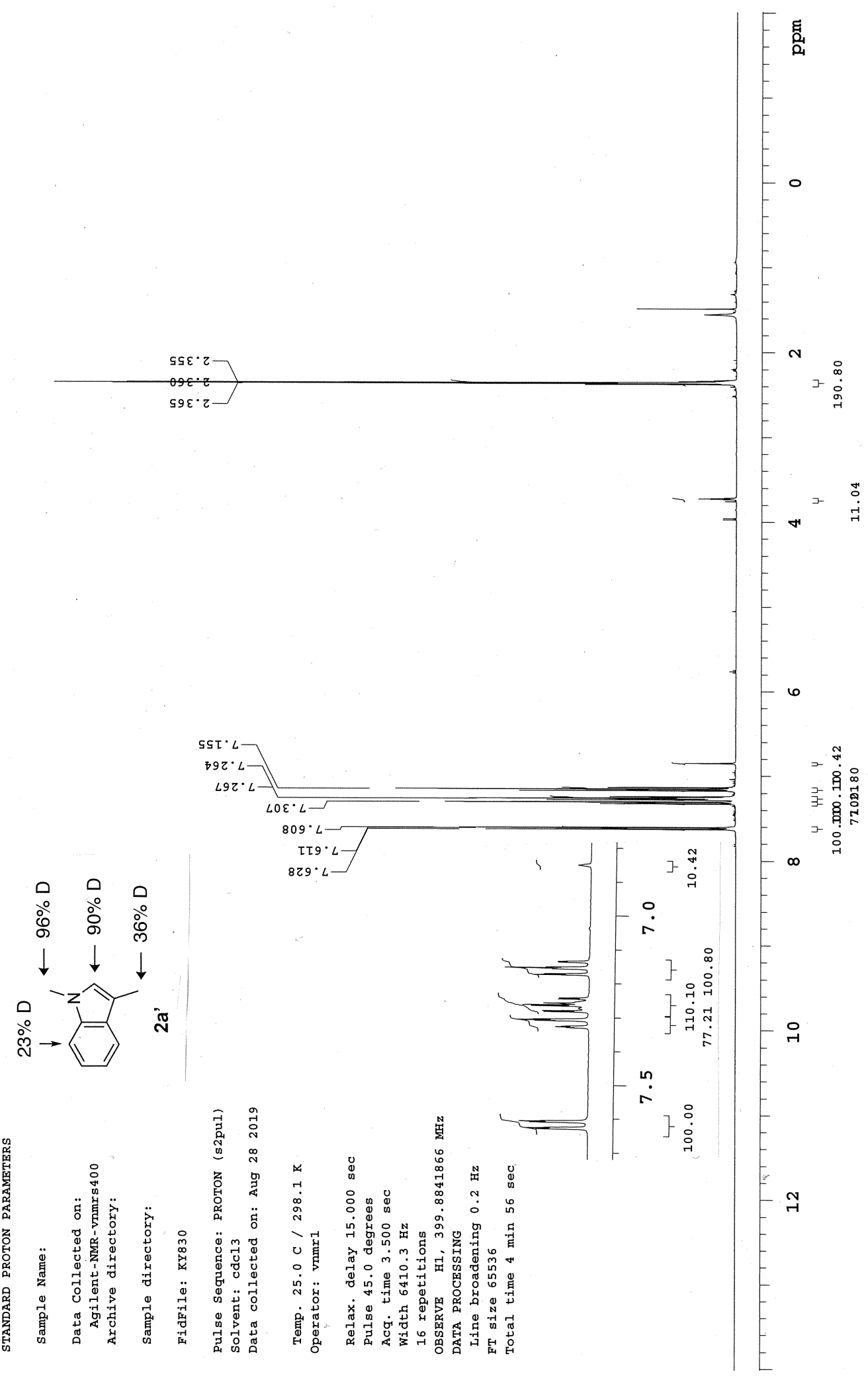

Figure S111. 'H NMR Spectrum of 2a' $\left(400 \mathrm{MHz}, \mathrm{CDCl}_{3}\right)$ 


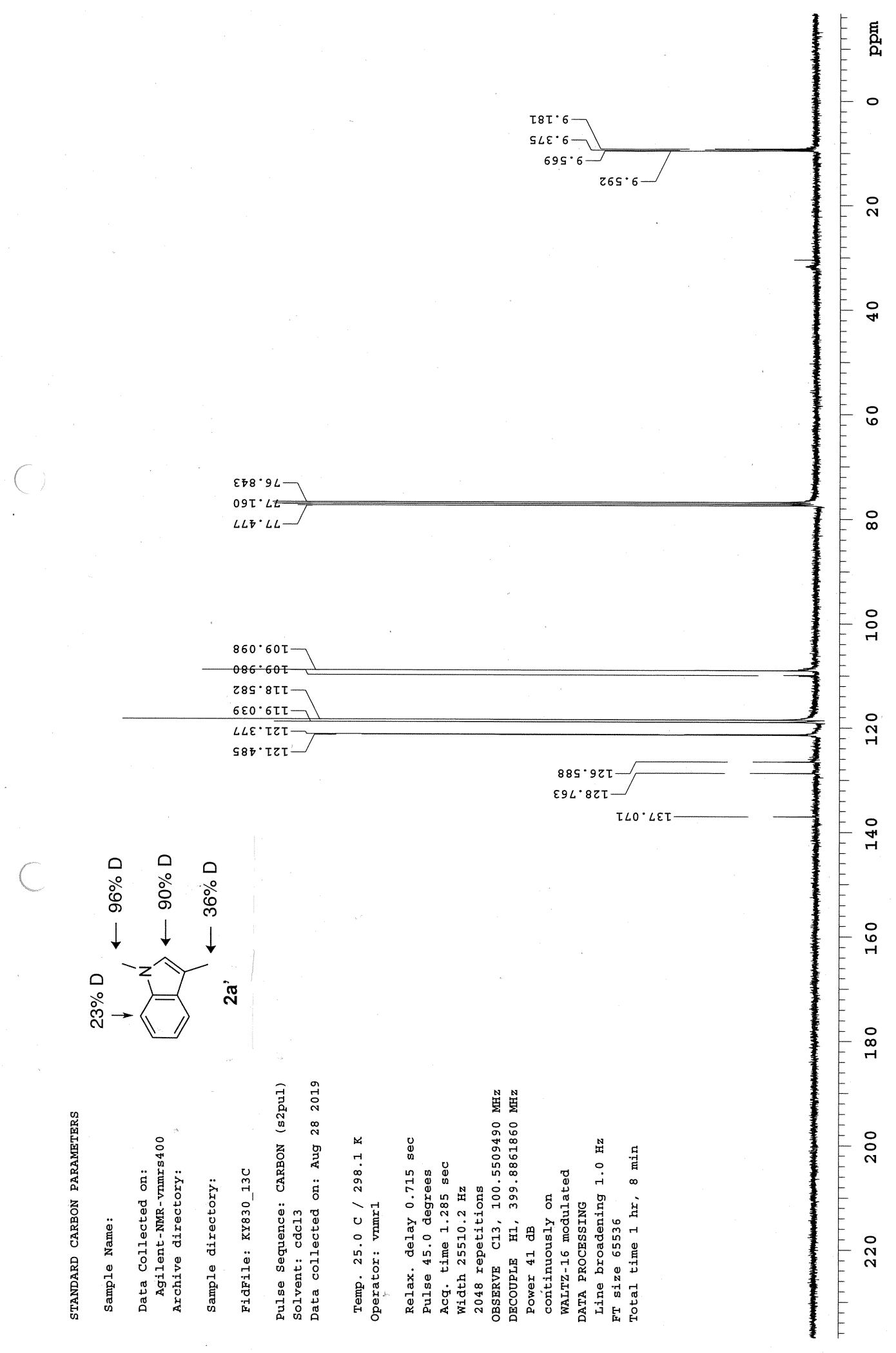

Figure S112. ${ }^{13} \mathrm{C}$ NMR Spectrum of $\mathbf{2 a}$ ' $\left(101 \mathrm{MHz}, \mathrm{CDCl}_{3}\right)$ 


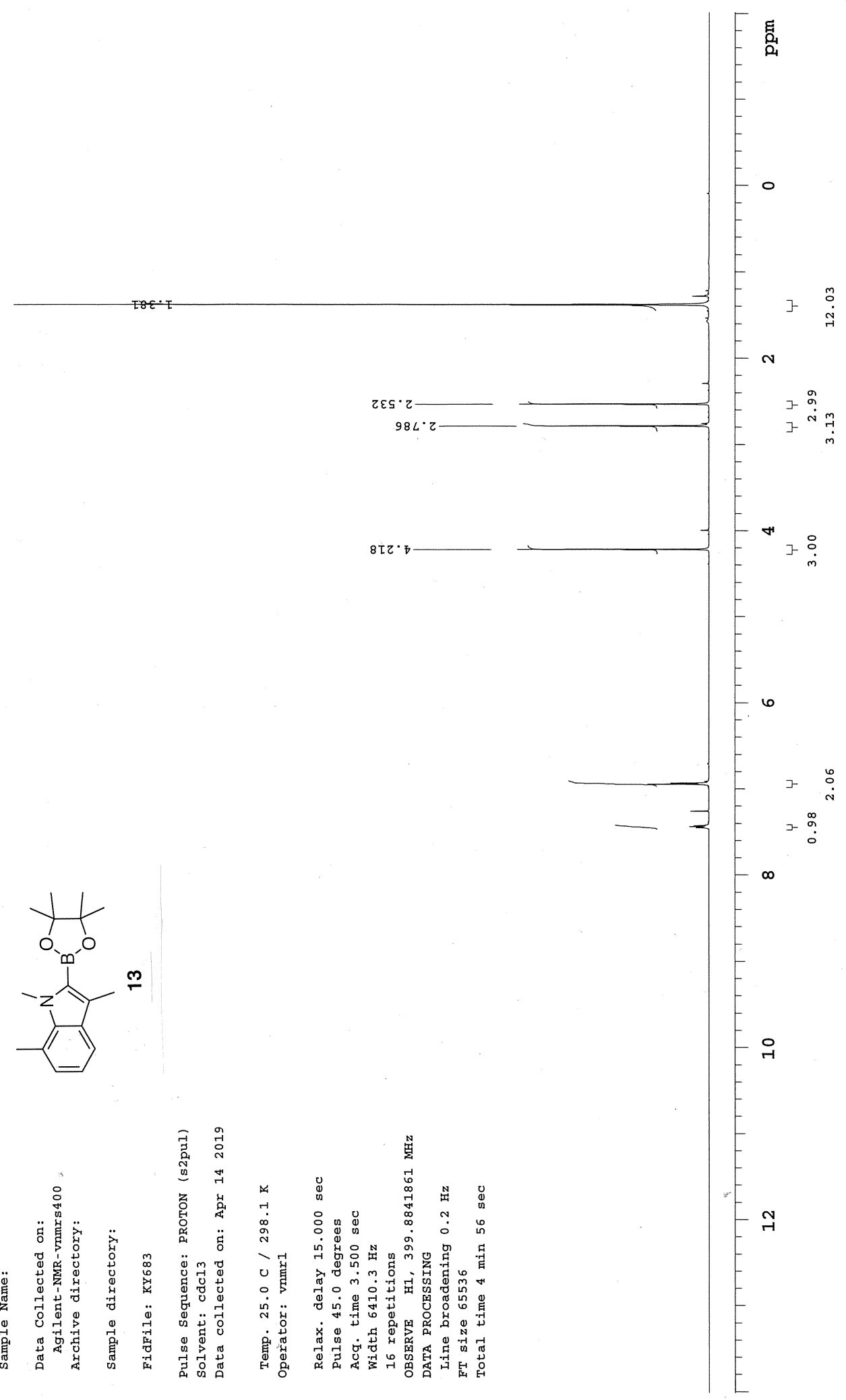

Figure S113. ${ }^{1} \mathrm{H}$ NMR Spectrum of $13\left(400 \mathrm{MHz}, \mathrm{CDCl}_{3}\right)$ 


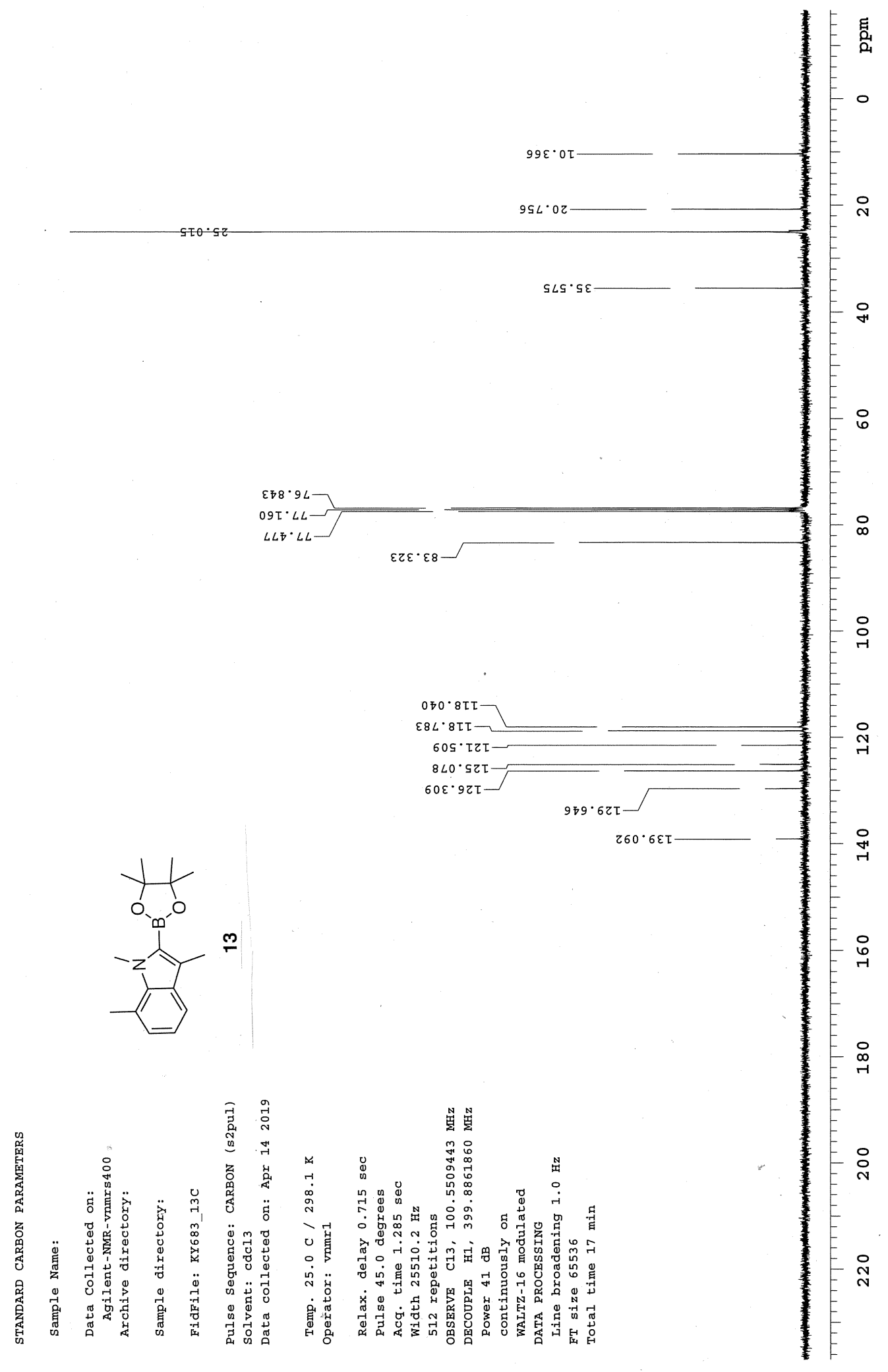

Figure S114. ${ }^{13} \mathrm{C}$ NMR Spectrum of $13\left(101 \mathrm{MHz}, \mathrm{CDCl}_{3}\right)$ 


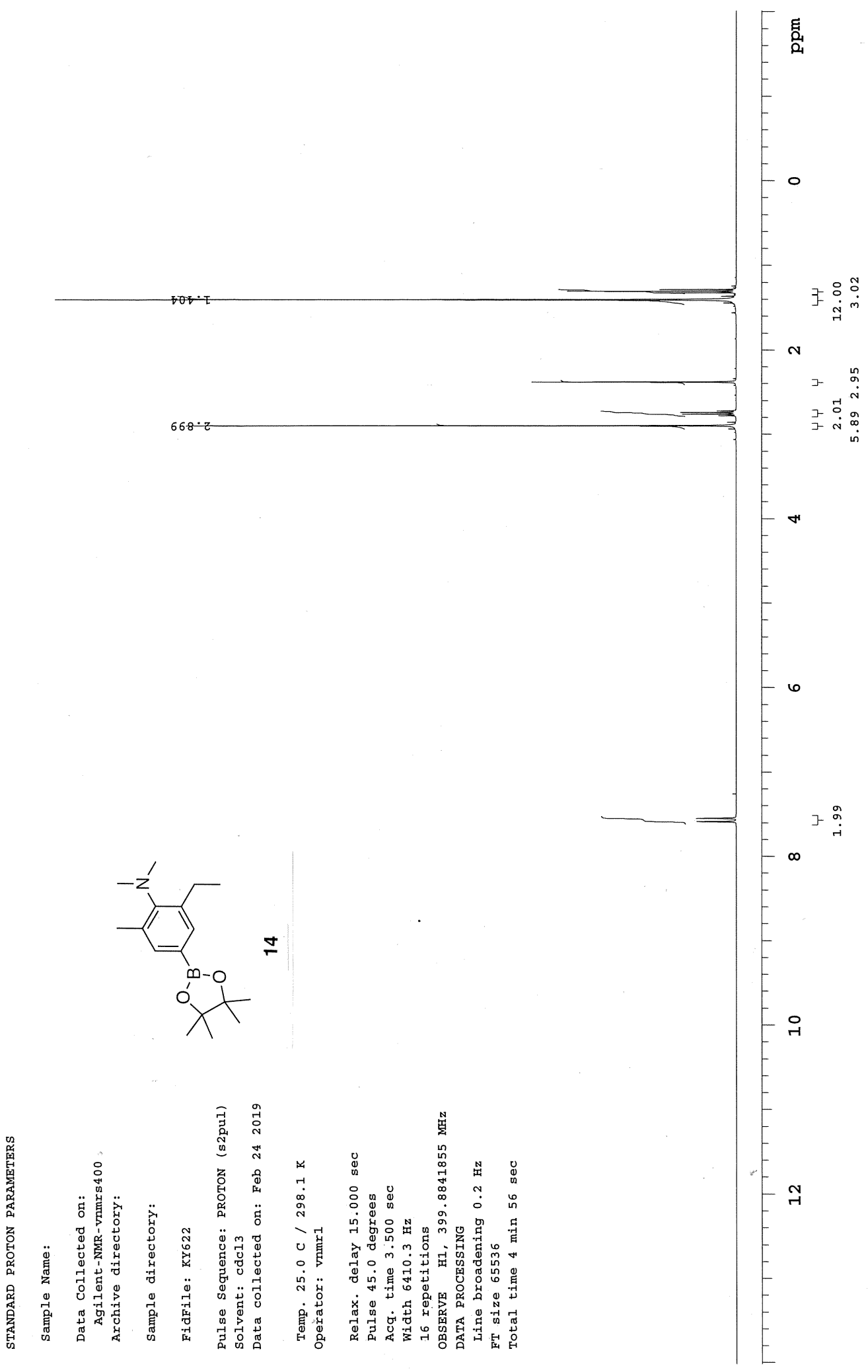

Figure S115. ${ }^{1} \mathrm{H}$ NMR Spectrum of $14\left(400 \mathrm{MHz}, \mathrm{CDCl}_{3}\right)$ 


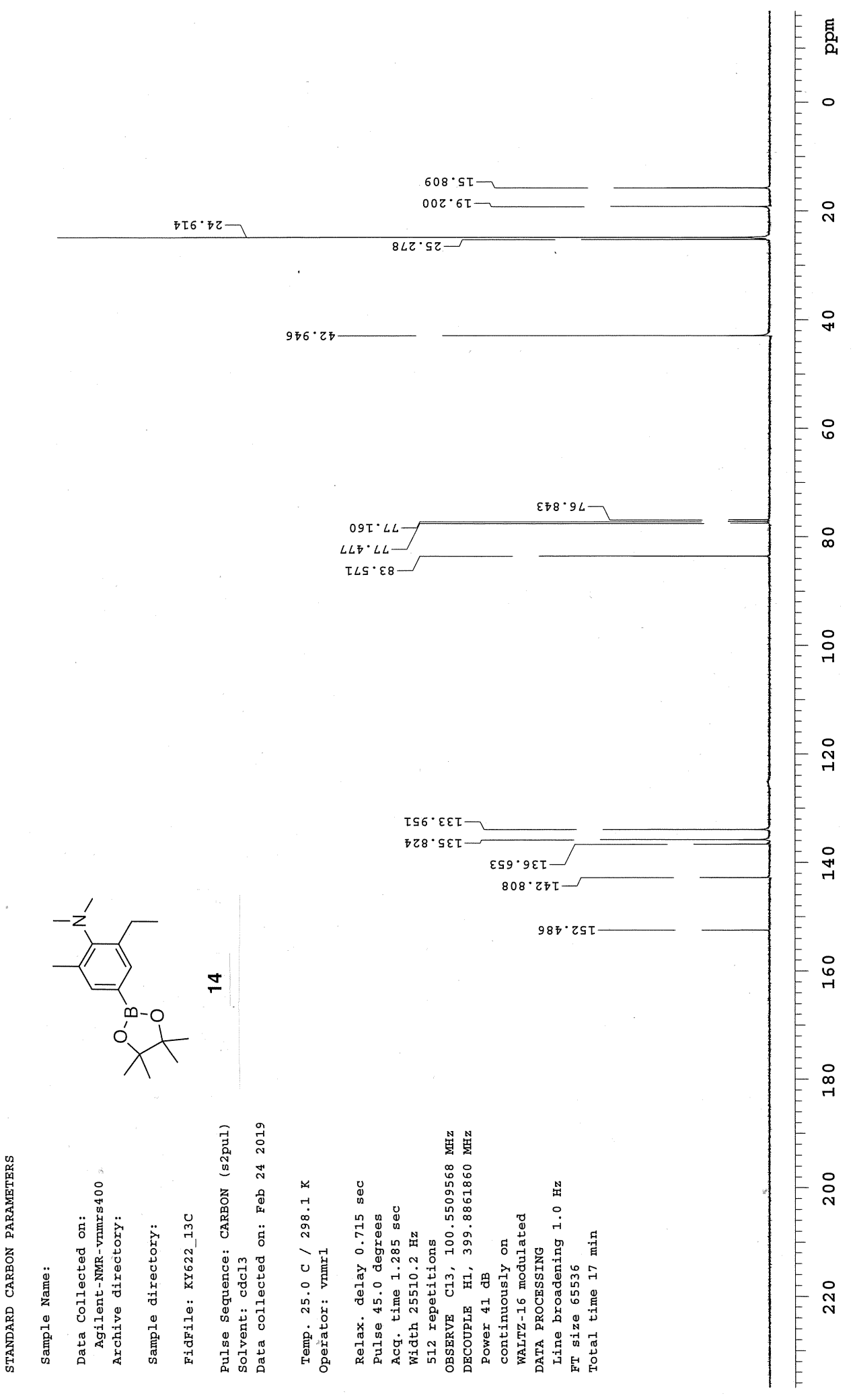

Figure S116. ${ }^{13} \mathrm{C}$ NMR Spectrum of $14\left(101 \mathrm{MHz}, \mathrm{CDCl}_{3}\right)$ 


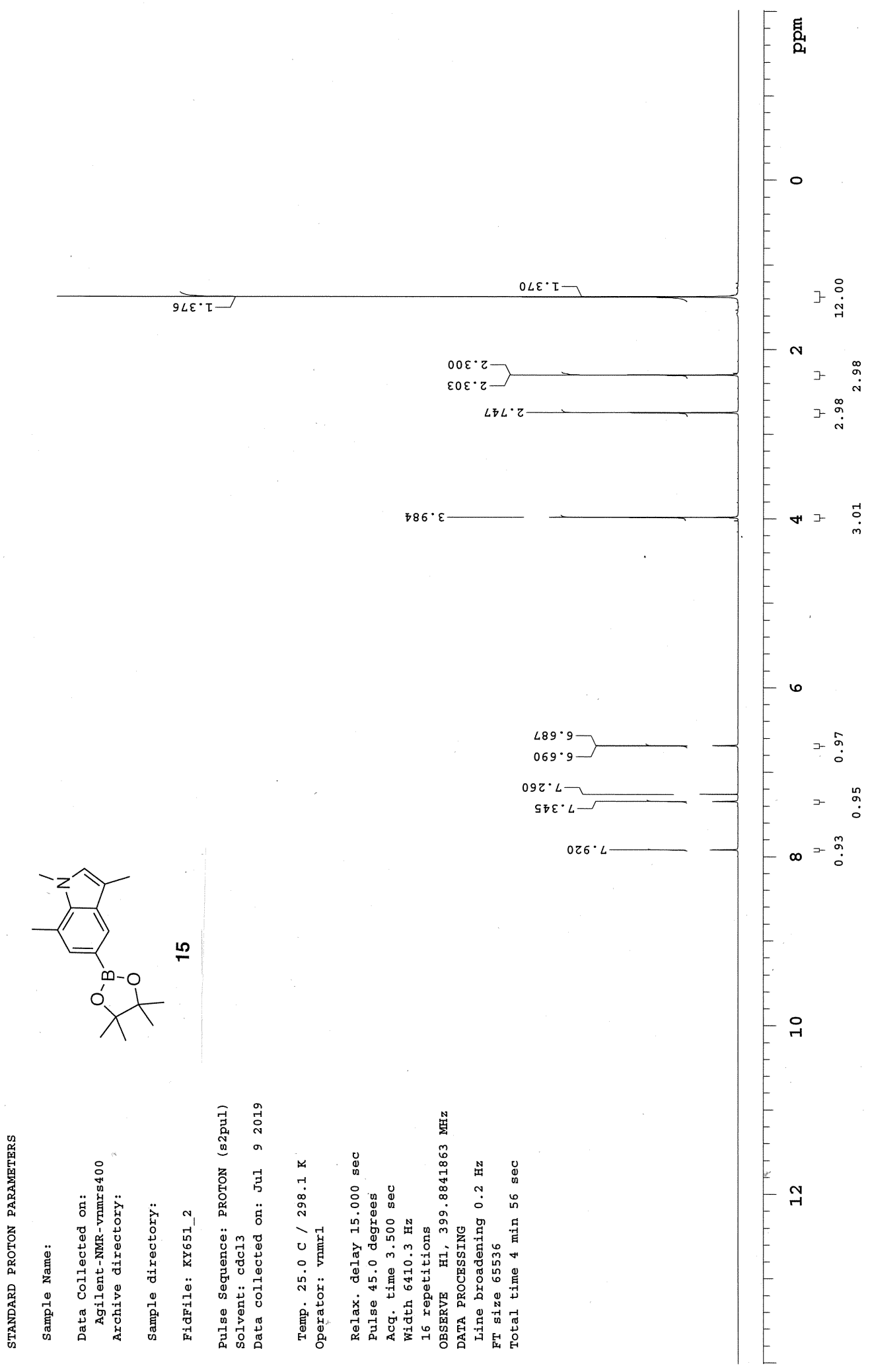

Figure S117. ${ }^{1} \mathrm{H}$ NMR Spectrum of $15\left(400 \mathrm{MHz}, \mathrm{CDCl}_{3}\right)$ 


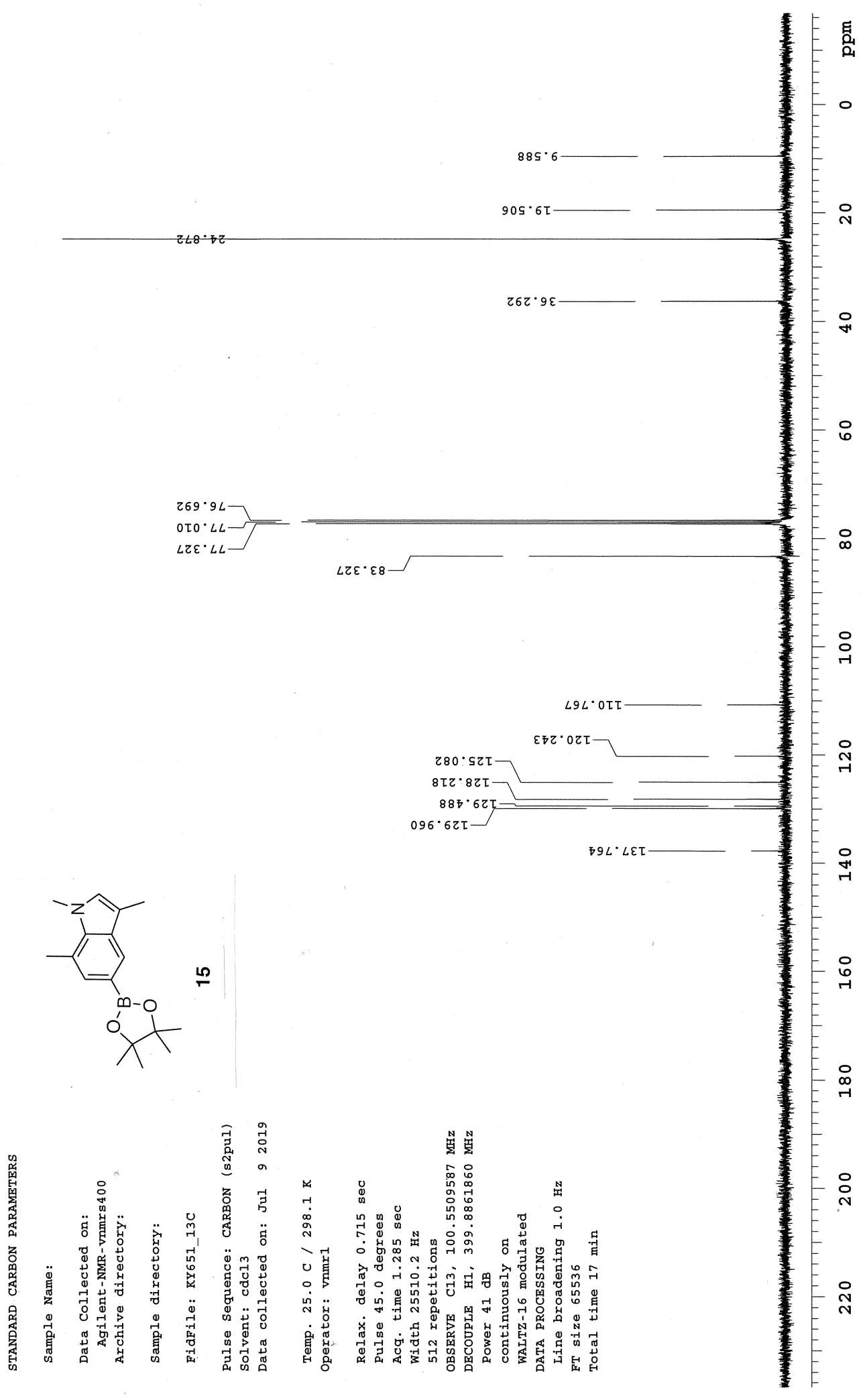

Figure S118. ${ }^{13} \mathrm{C}$ NMR Spectrum of $15\left(101 \mathrm{MHz}, \mathrm{CDCl}_{3}\right)$ 


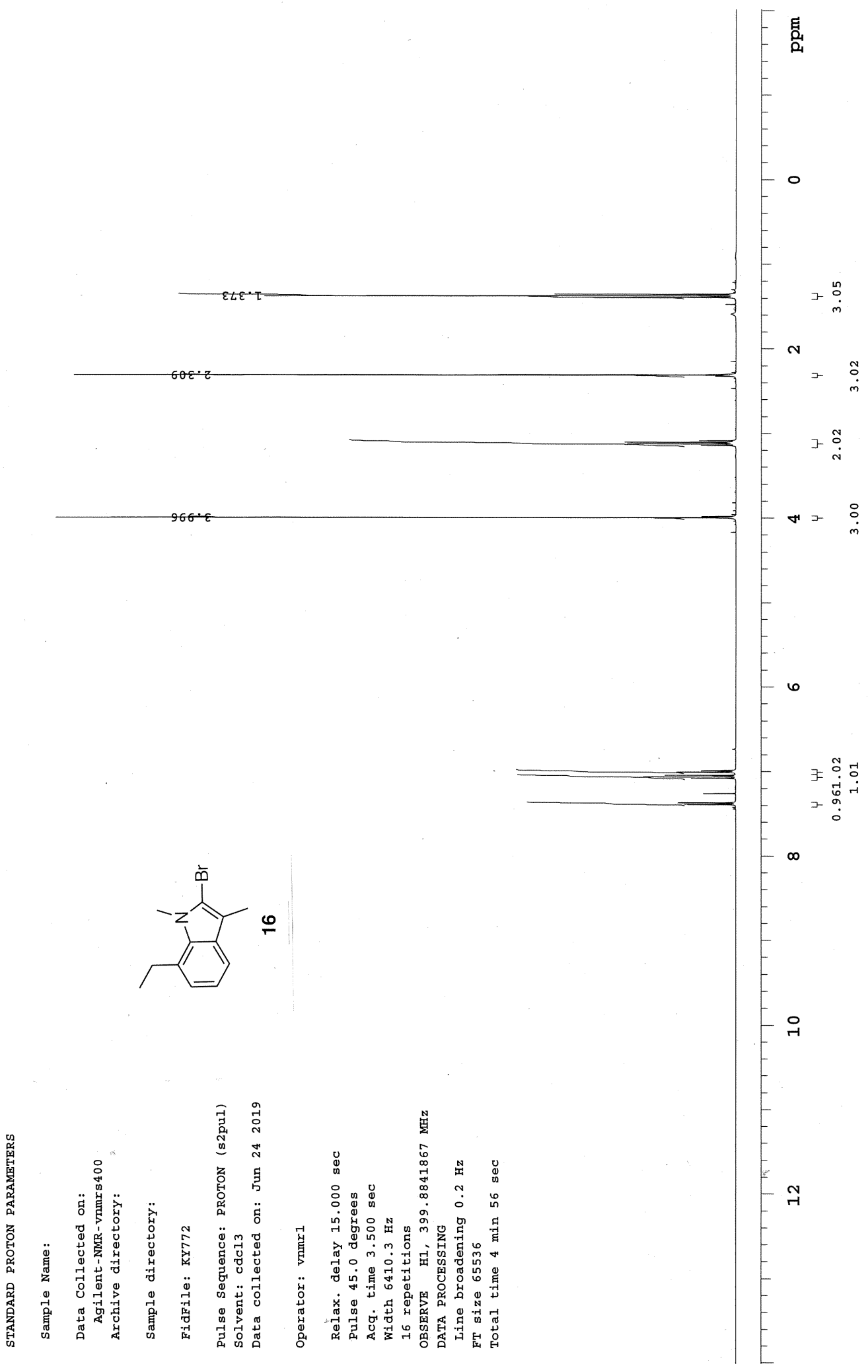

Figure S119. ${ }^{1} \mathrm{H}$ NMR Spectrum of $16\left(400 \mathrm{MHz}, \mathrm{CDCl}_{3}\right)$ 


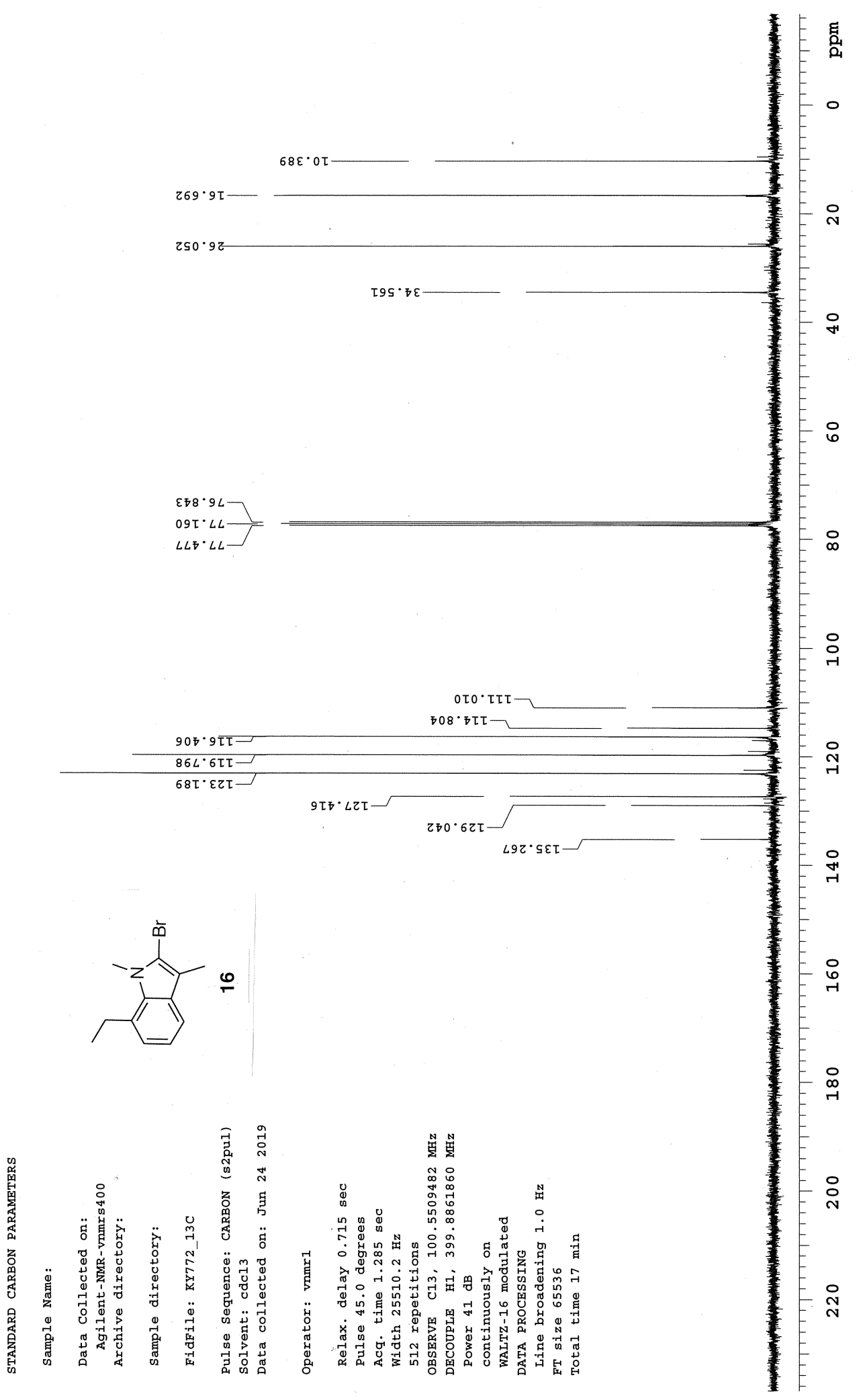

Figure S120. ${ }^{13} \mathrm{C}$ NMR Spectrum of $16\left(101 \mathrm{MHz}, \mathrm{CDCl}_{3}\right)$ 


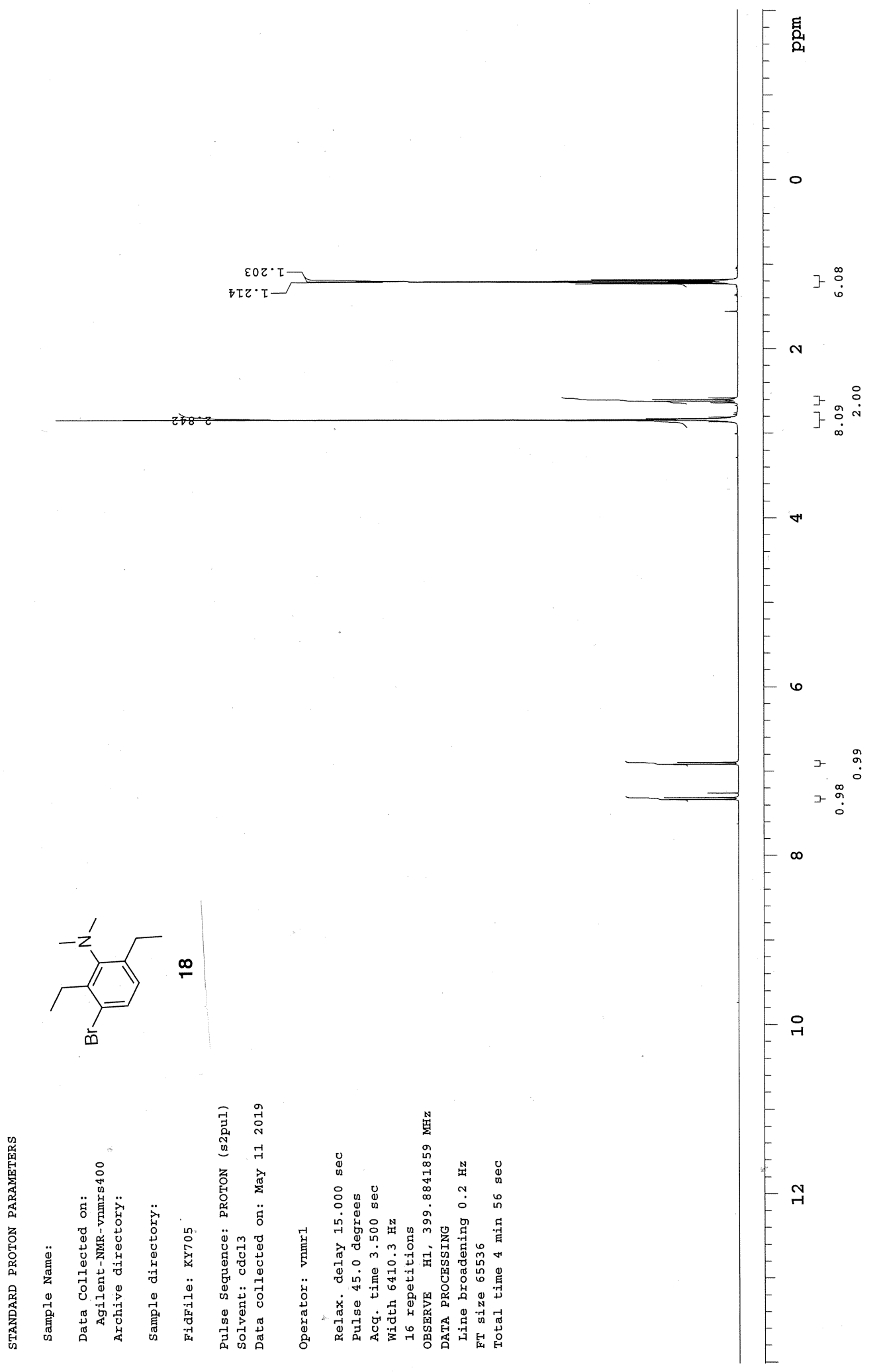

Figure S121. ${ }^{1} \mathrm{H}$ NMR Spectrum of $18\left(400 \mathrm{MHz}, \mathrm{CDCl}_{3}\right)$ 


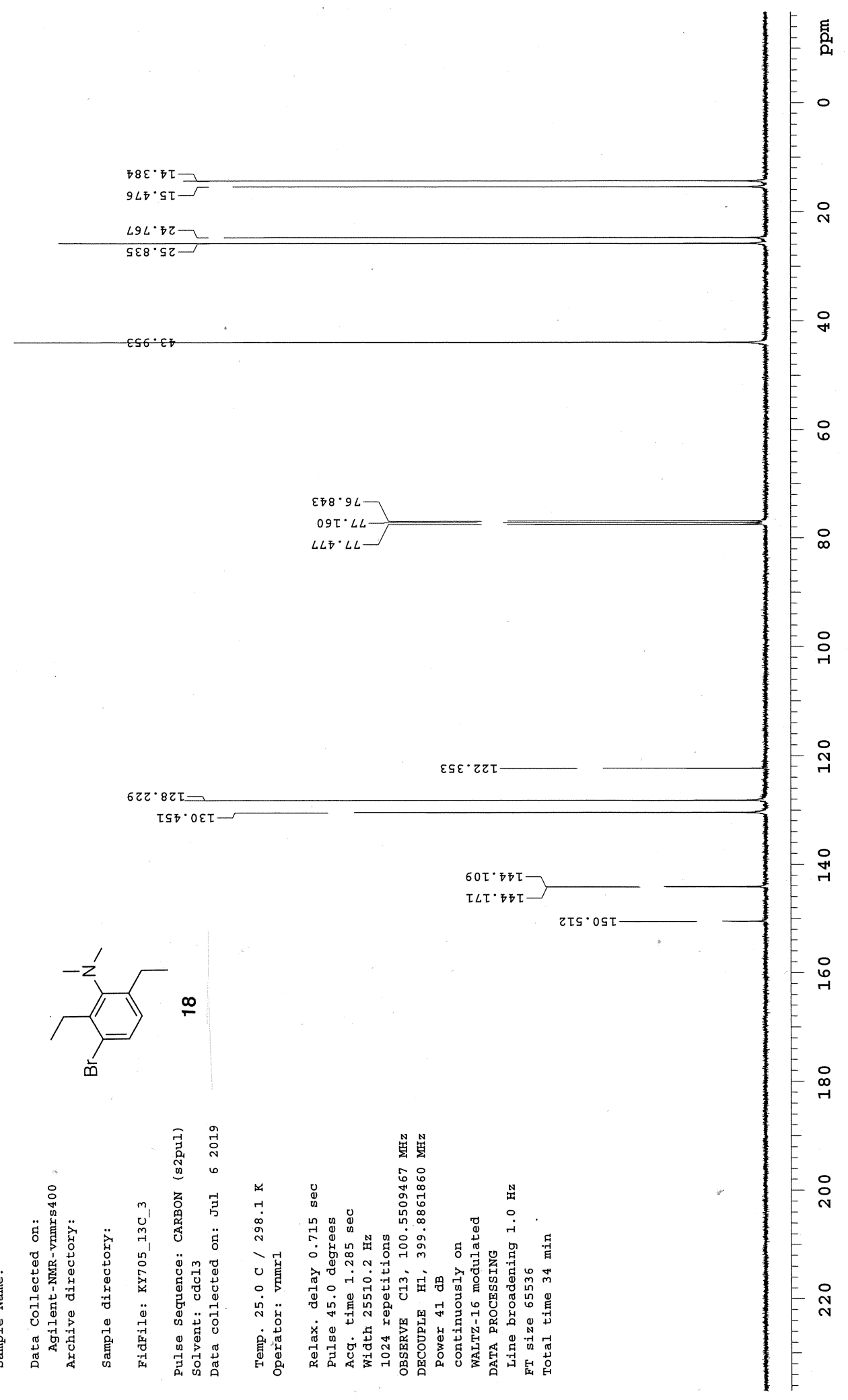

Figure S122. ${ }^{13} \mathrm{C}$ NMR Spectrum of $18\left(101 \mathrm{MHz}, \mathrm{CDCl}_{3}\right)$ 


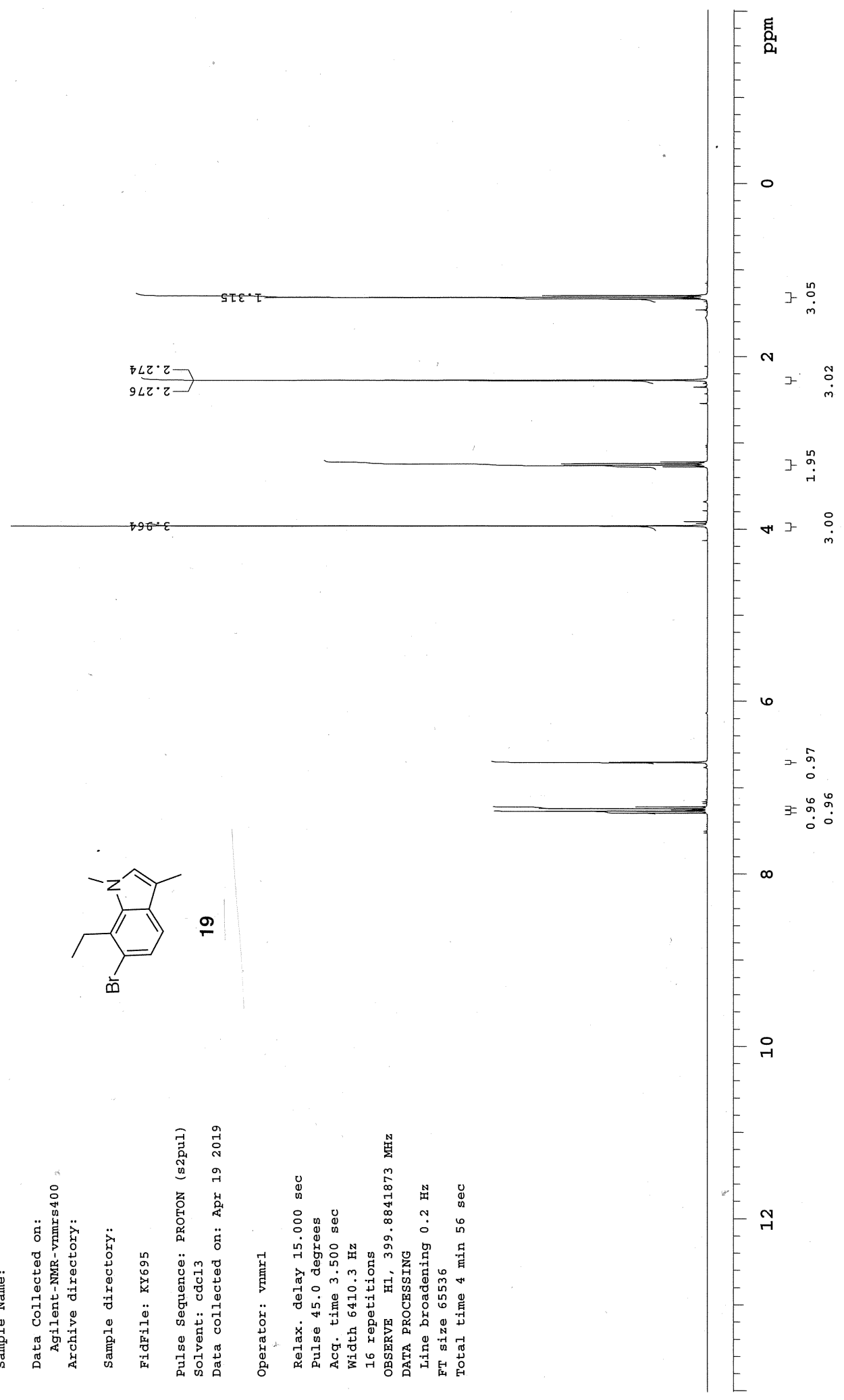

Figure S123. ${ }^{1} \mathrm{H}$ NMR Spectrum of $19\left(400 \mathrm{MHz}, \mathrm{CDCl}_{3}\right)$ 


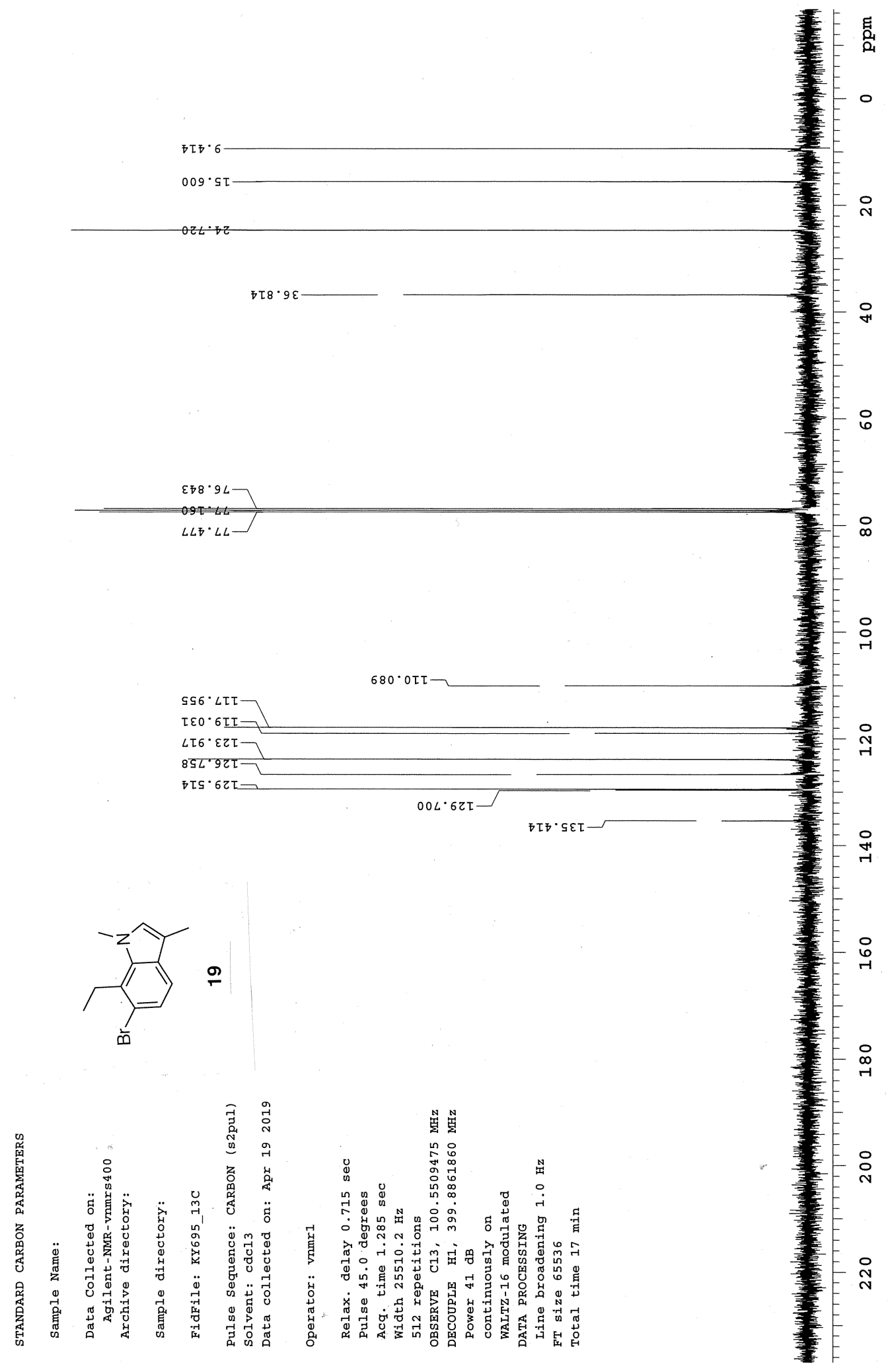

Figure S124. ${ }^{13} \mathrm{C}$ NMR Spectrum of $19\left(101 \mathrm{MHz}, \mathrm{CDCl}_{3}\right)$ 


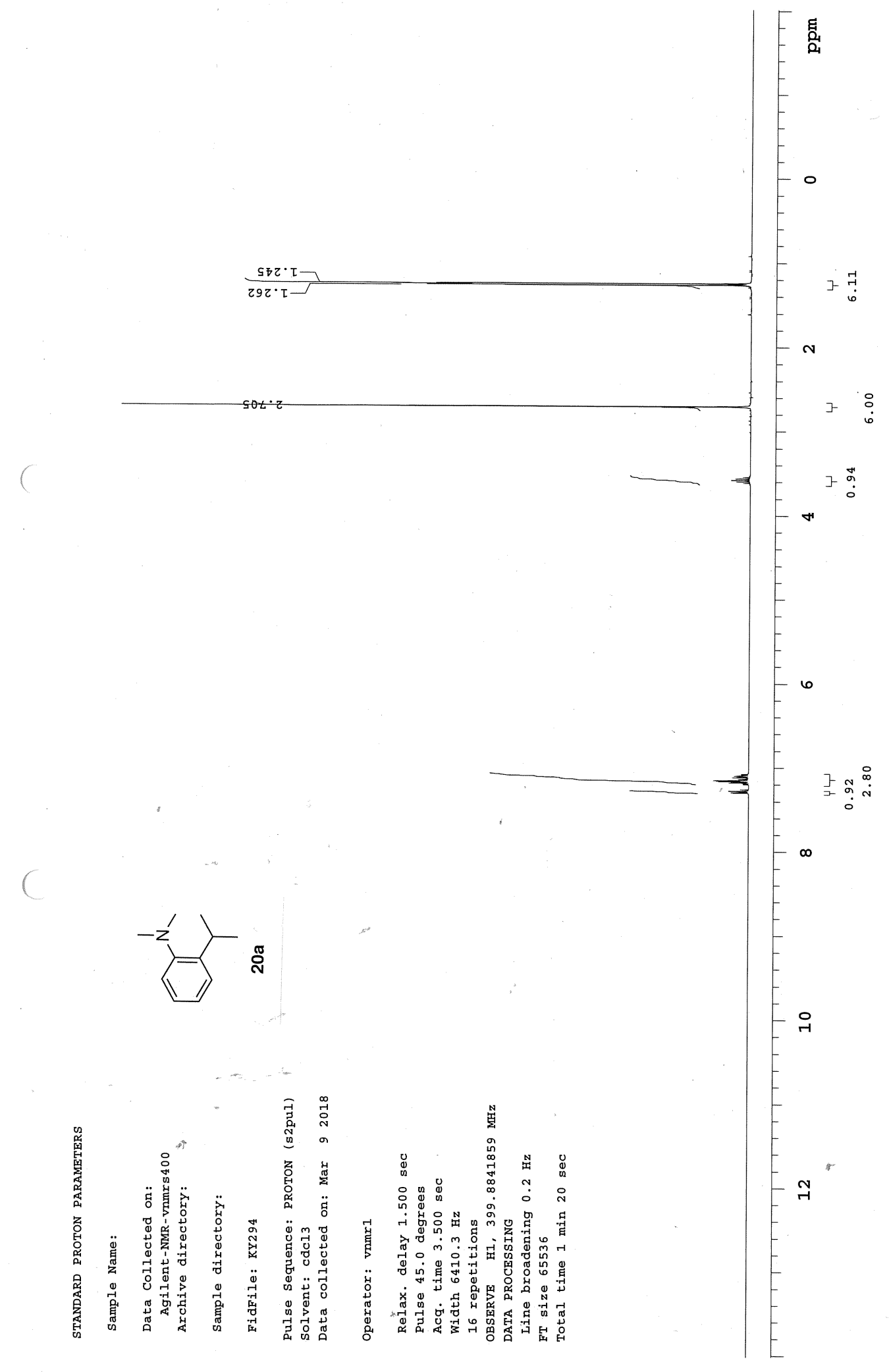

Figure S125. ${ }^{1} \mathrm{H}$ NMR Spectrum of 20a $\left(400 \mathrm{MHz}, \mathrm{CDCl}_{3}\right)$ 


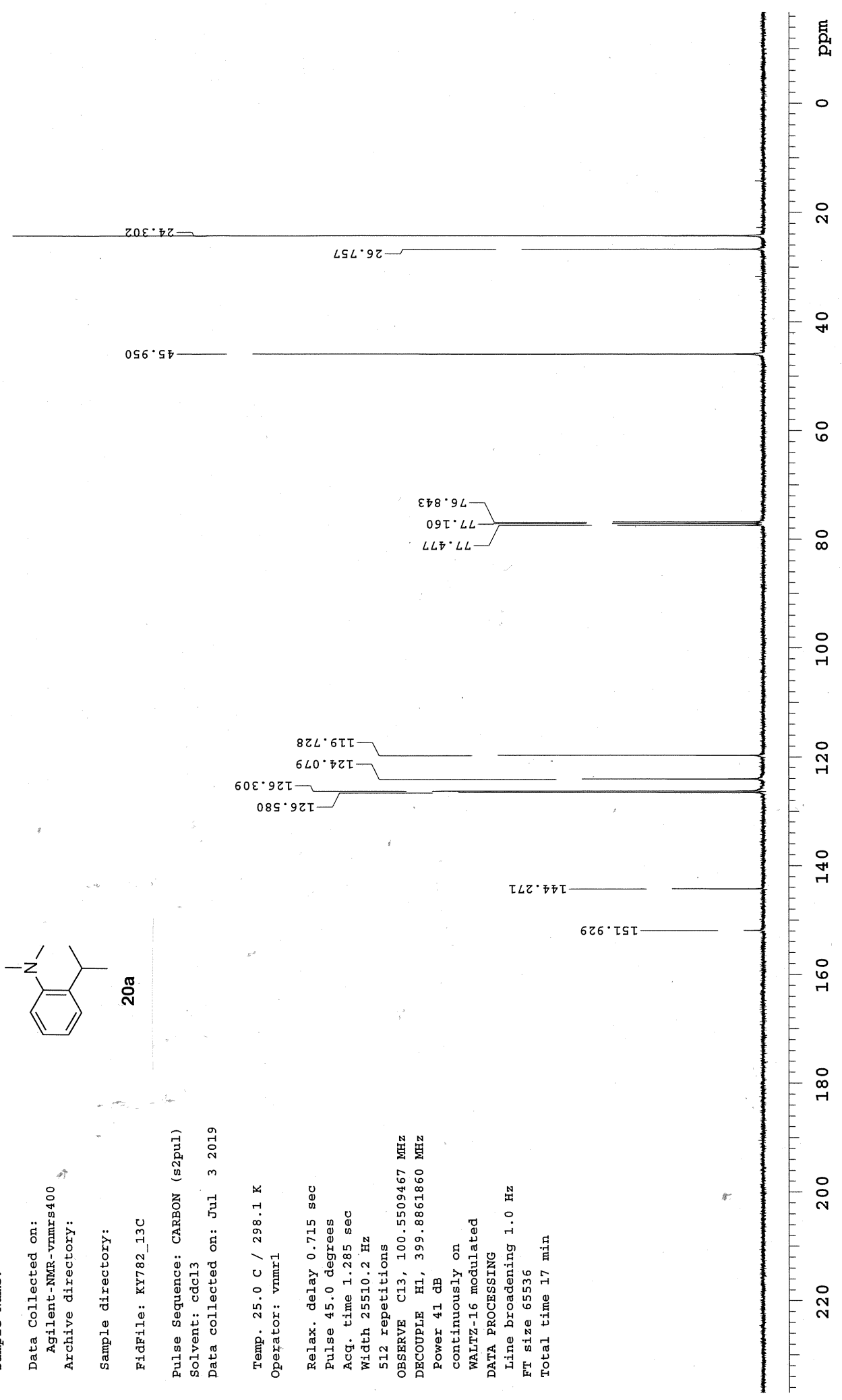

Figure S126. ${ }^{13} \mathrm{C}$ NMR Spectrum of 20a $\left(101 \mathrm{MHz}, \mathrm{CDCl}_{3}\right)$ 


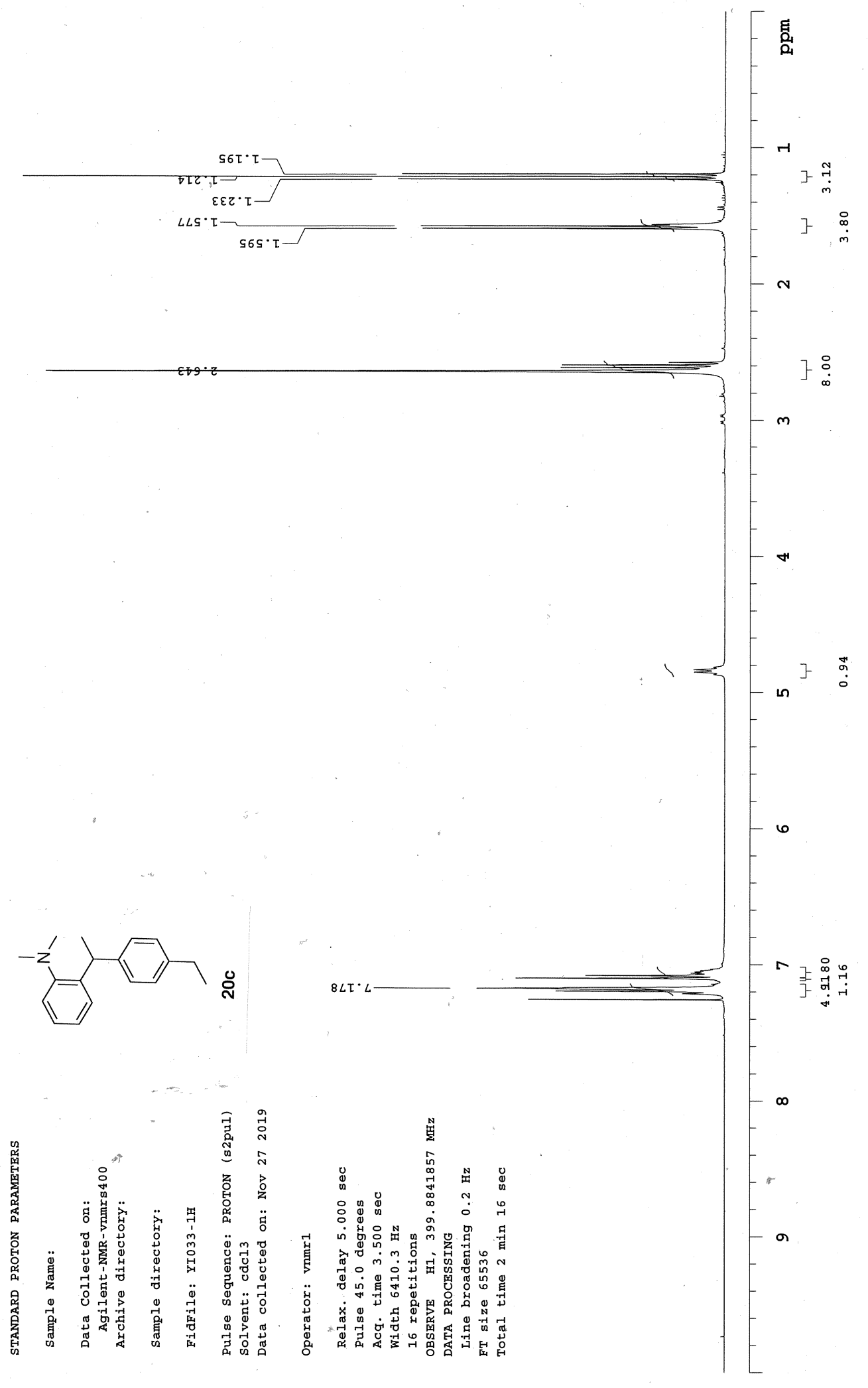

Figure S127. ${ }^{1} \mathrm{H}$ NMR Spectrum of 20c $\left(400 \mathrm{MHz}, \mathrm{CDCl}_{3}\right)$ 


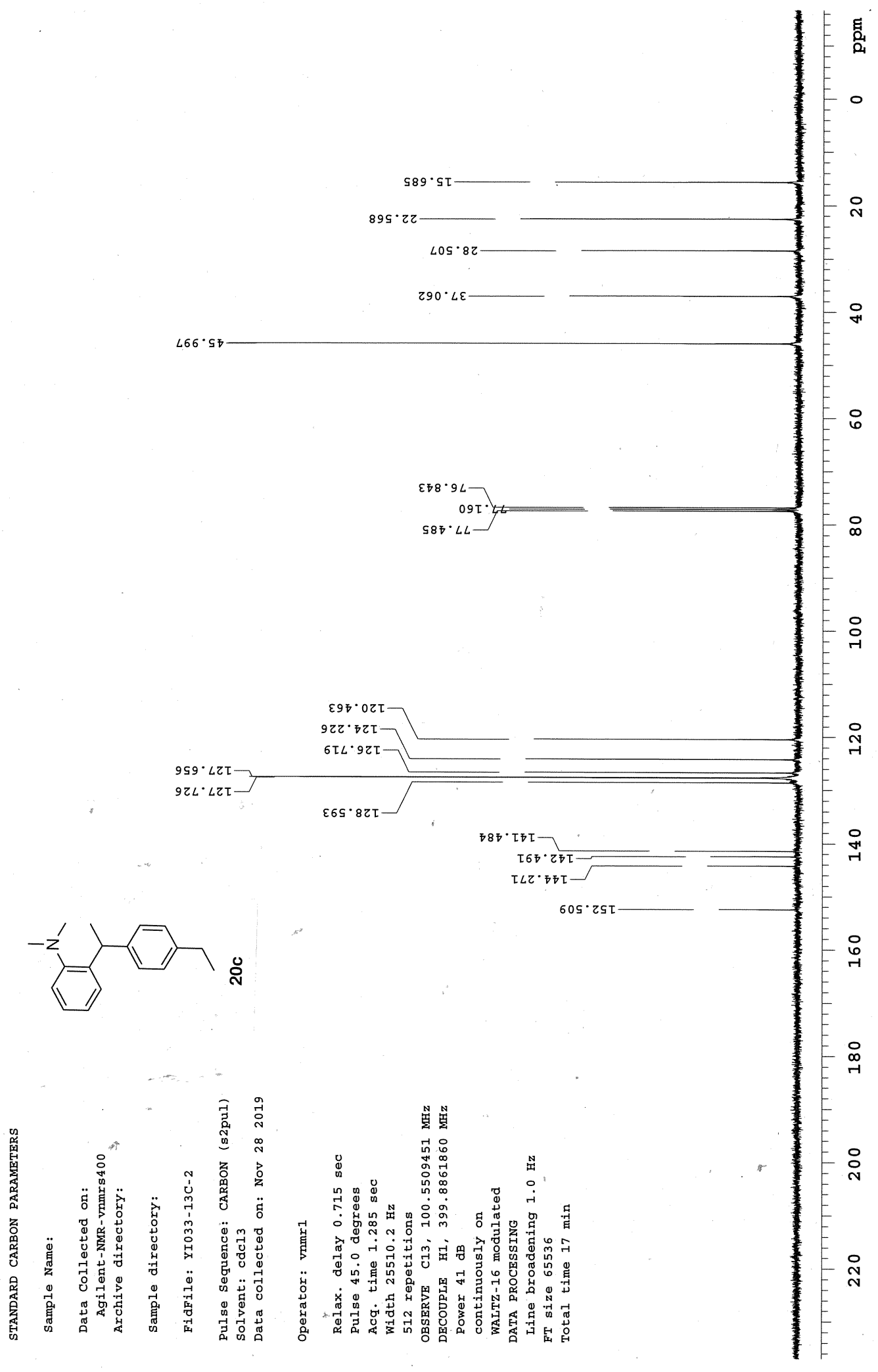

Figure S128. ${ }^{13} \mathrm{C}$ NMR Spectrum of $20 \mathrm{c}\left(101 \mathrm{MHz}, \mathrm{CDCl}_{3}\right)$ 


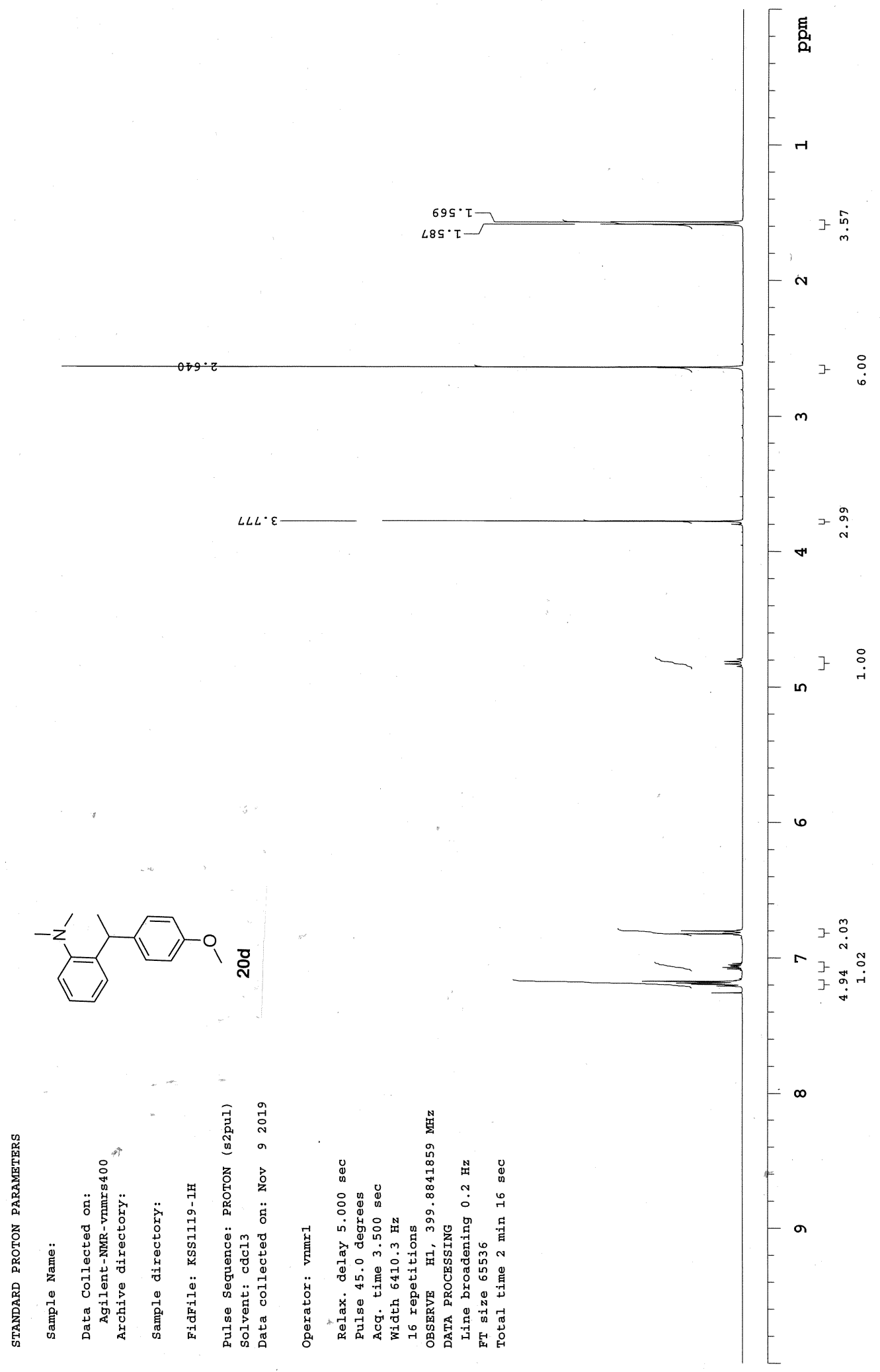

Figure S129. ${ }^{1} \mathrm{H}$ NMR Spectrum of $20 d\left(400 \mathrm{MHz}, \mathrm{CDCl}_{3}\right)$ 


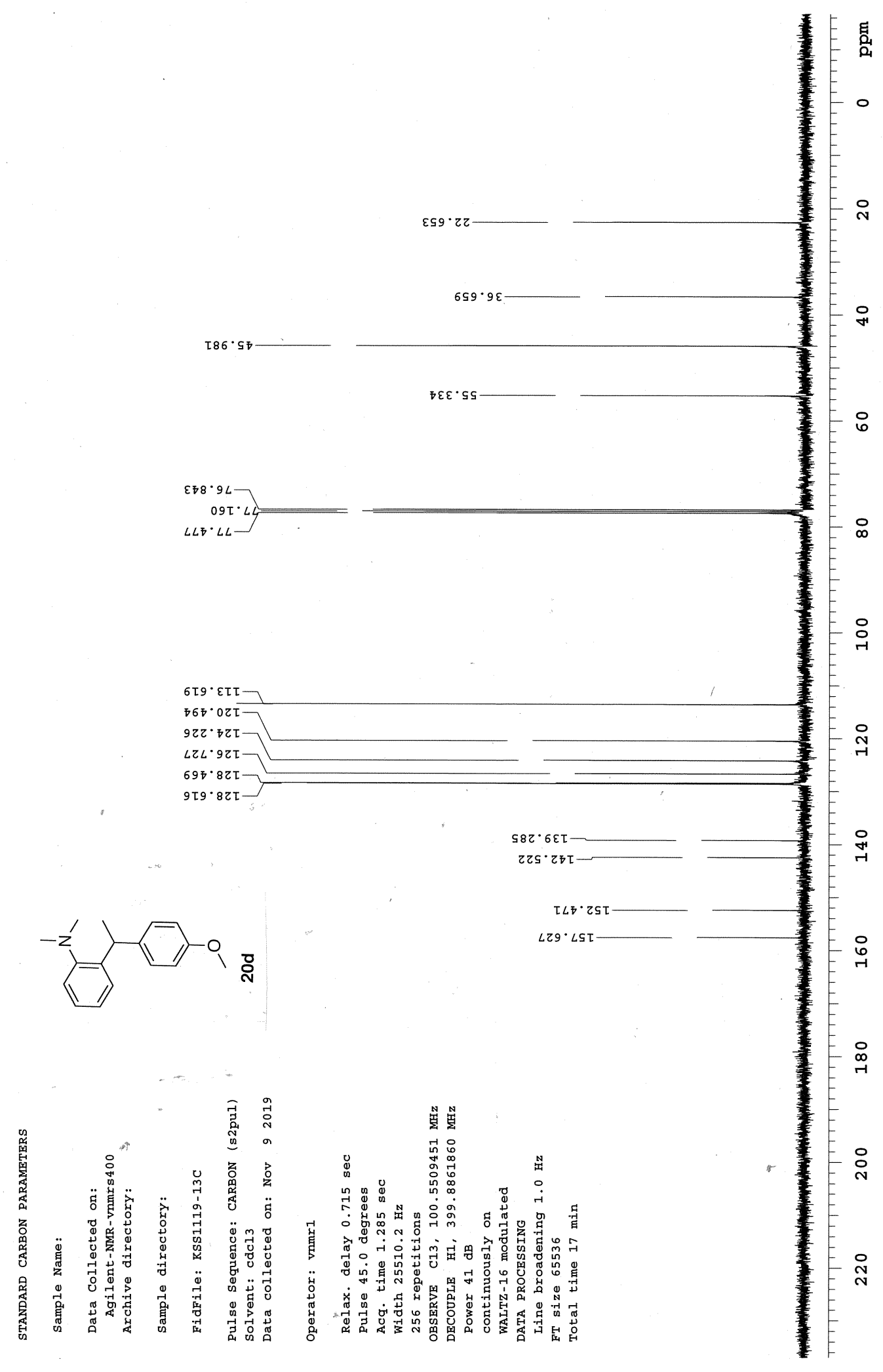

Figure S130. ${ }^{13} \mathrm{C}$ NMR Spectrum of $20 d\left(101 \mathrm{MHz}, \mathrm{CDCl}_{3}\right)$ 


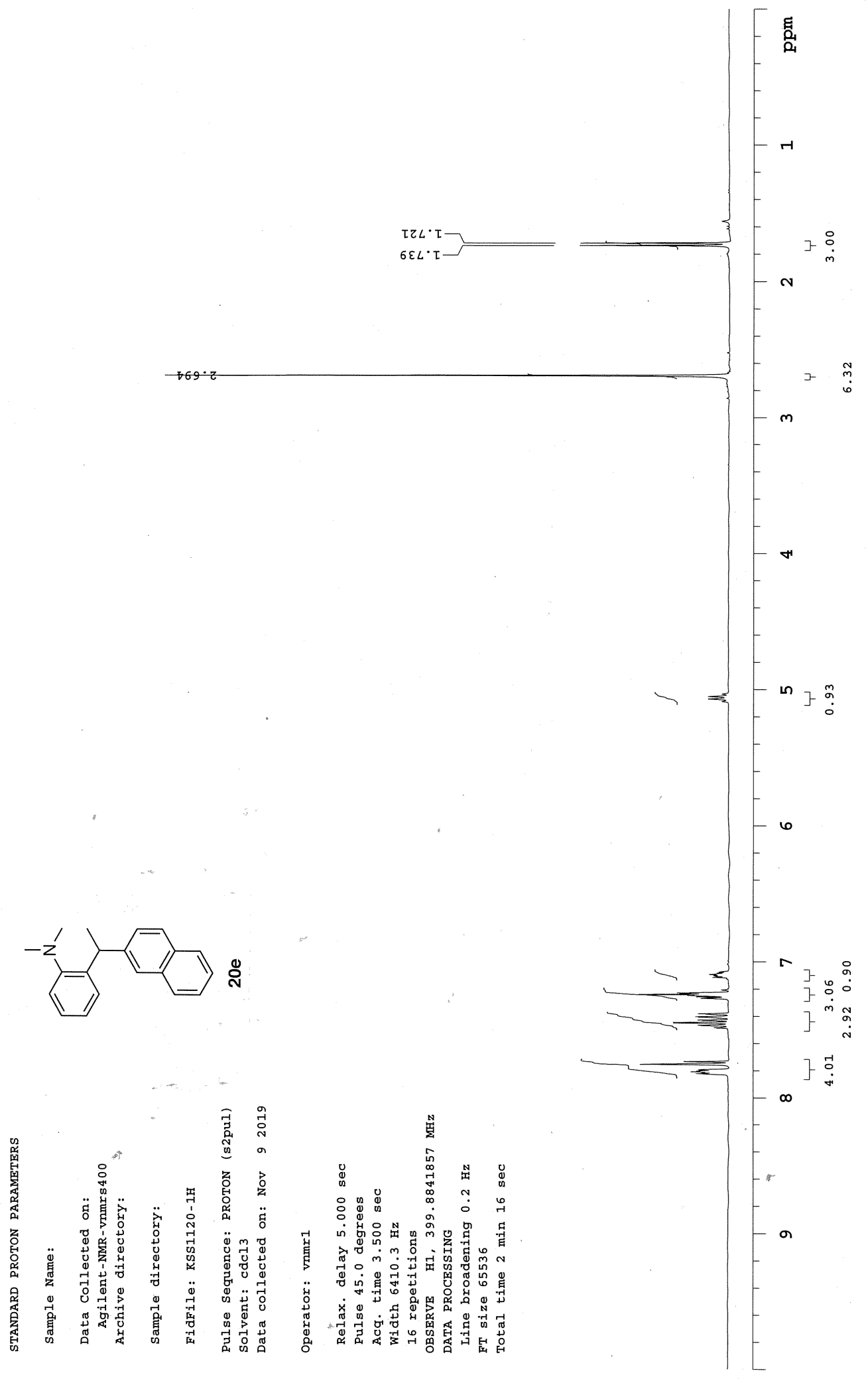

Figure S131. ${ }^{1} \mathrm{H}$ NMR Spectrum of 20e (400 MHz, $\left.\mathrm{CDCl}_{3}\right)$ 


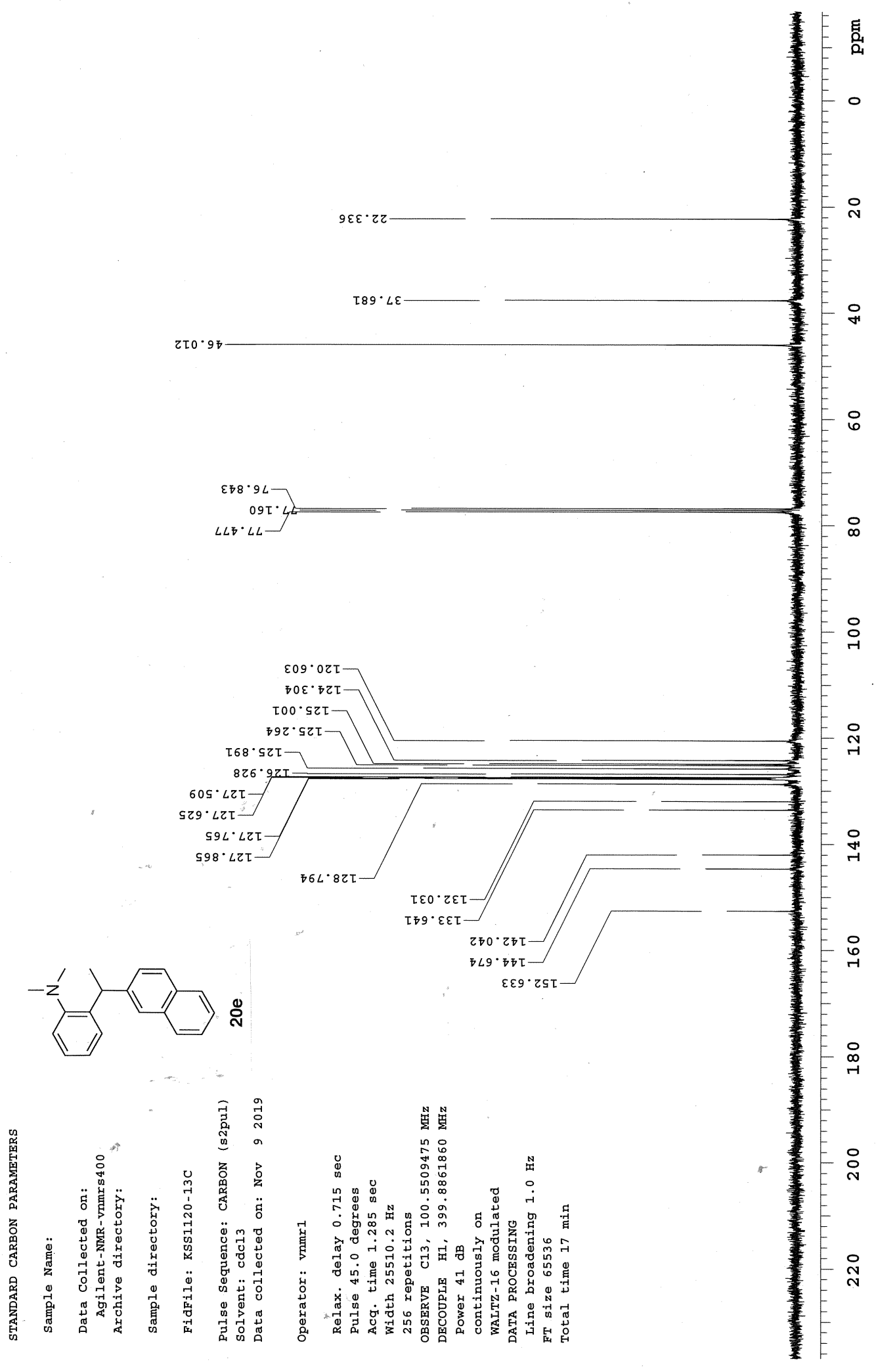

Figure S132. ${ }^{13} \mathrm{C}$ NMR Spectrum of $20 e\left(101 \mathrm{MHz}, \mathrm{CDCl}_{3}\right)$ 


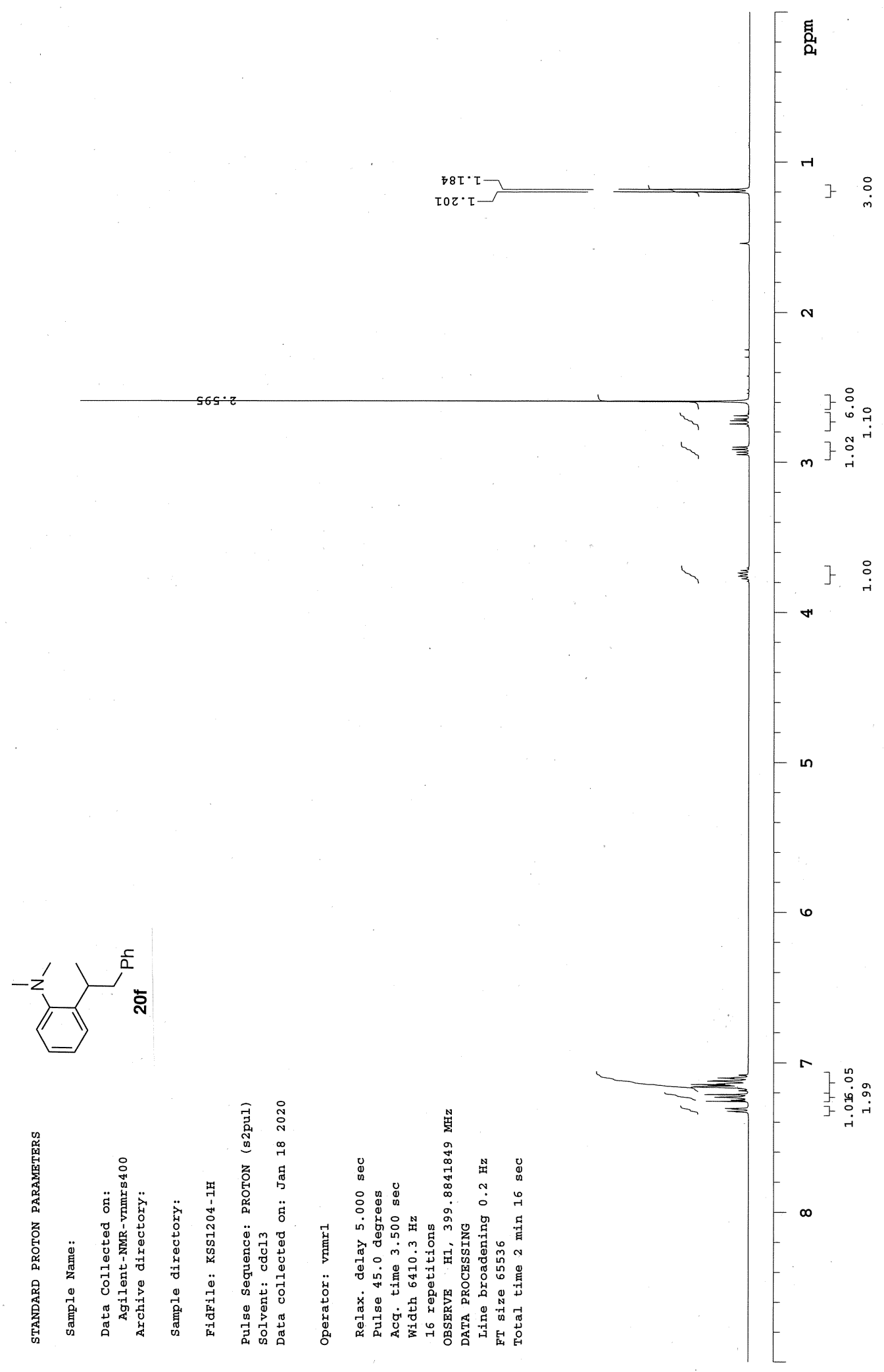

Figure S133. ${ }^{1} \mathrm{H}$ NMR Spectrum of $20 f\left(400 \mathrm{MHz}, \mathrm{CDCl}_{3}\right)$ 


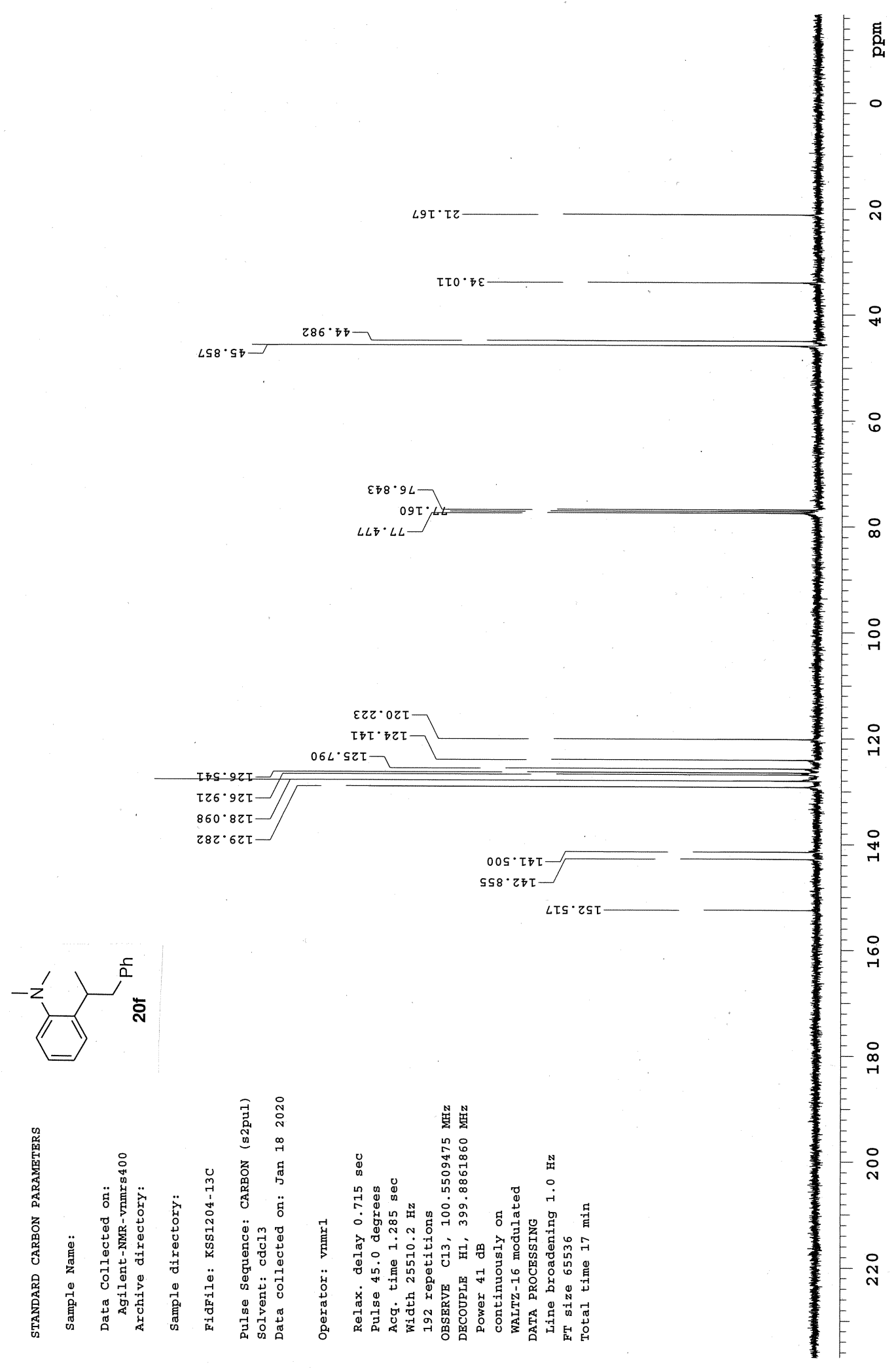

Figure S134. ${ }^{13} \mathrm{C}$ NMR Spectrum of $20 f\left(101 \mathrm{MHz}, \mathrm{CDCl}_{3}\right)$ 


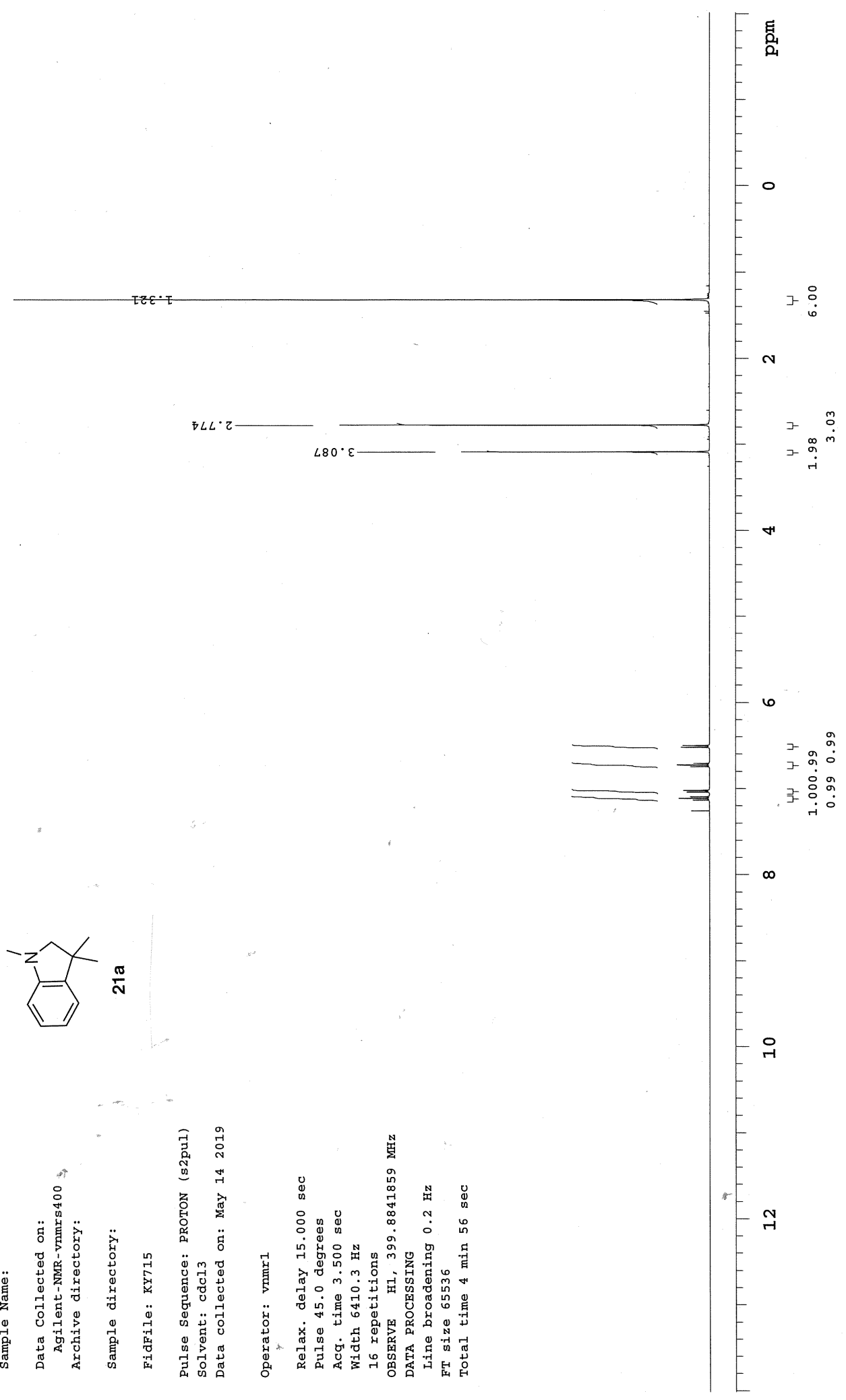

Figure S135. ${ }^{1} \mathrm{H}$ NMR Spectrum of 21 a $\left(400 \mathrm{MHz}, \mathrm{CDCl}_{3}\right)$ 


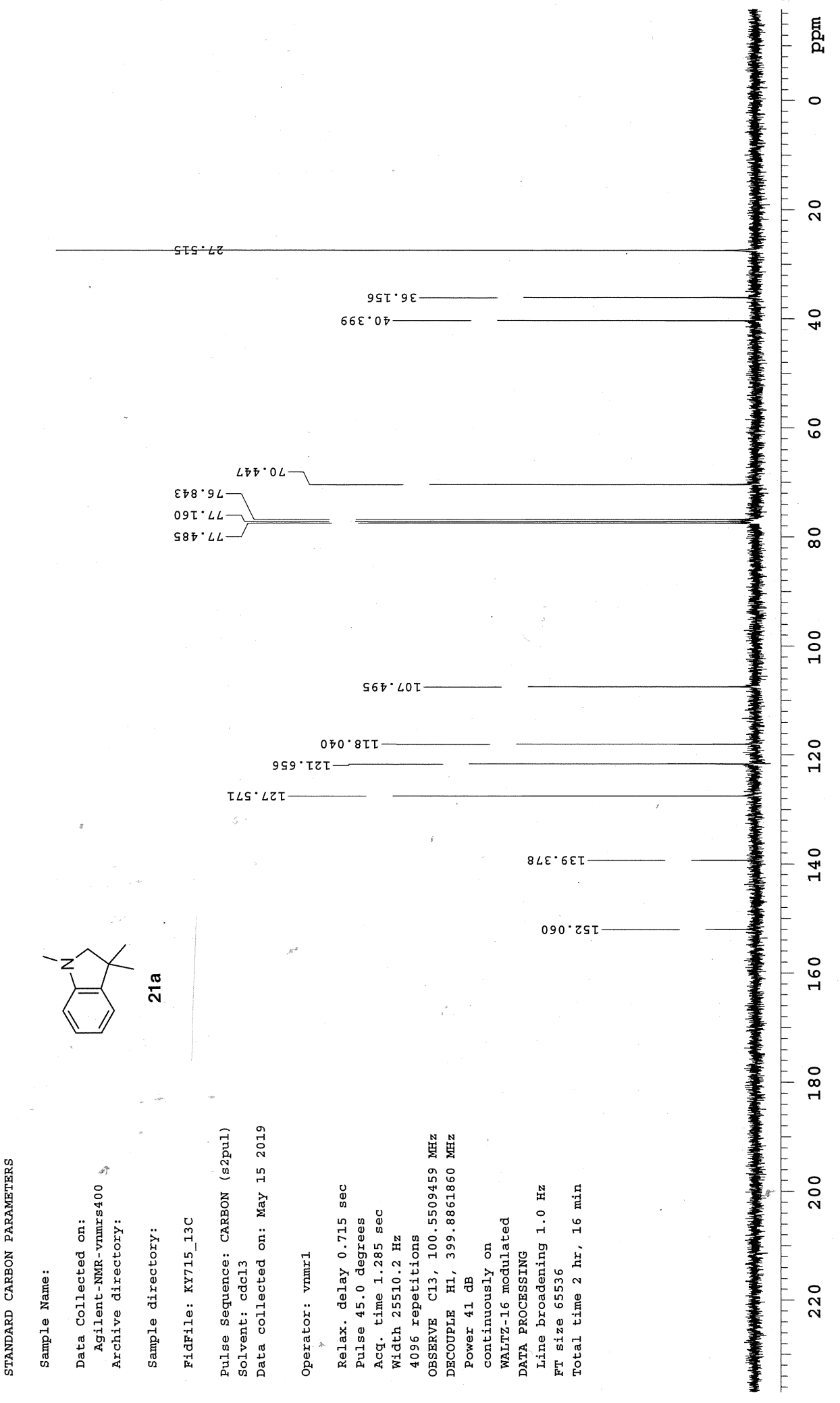

Figure S136. ${ }^{13} \mathrm{C}$ NMR Spectrum of 21a $\left(101 \mathrm{MHz}, \mathrm{CDCl}_{3}\right)$ 